\title{
Fiber optic SERS probes for remote sensing
}

by

\begin{abstract}
Aditya H Pandya
Master of Science, Biomedical Physics, Ryerson University, Toronto ON, Canada 2012

Bachelor of Engineering, Biomedical Engineering, Gujarat University, Gujarat, India 2008
\end{abstract}

\author{
A thesis \\ presented to Ryerson University \\ in partial fulfilment of the \\ requirements for the degree of \\ Doctor of Philosophy \\ In the Program of \\ Biomedical Physics
}

Toronto, Ontario, Canada 2019

(C) Copyright by Aditya Pandya 2019 


\section{Author's declaration for electronic submission of a dissertation}

I hereby declare that I am the sole author of this dissertation. This is a true copy of the dissertation, including any required final revisions, as accepted by my examiners.

I authorize Ryerson University to lend this dissertation to other institutions or individuals for the purpose of scholarly research.

I further authorize Ryerson University to reproduce this dissertation by photocopying or by other means, in total or in part, at the request of other institutions or individuals for the purpose of scholarly research.

I understand that my dissertation may be made electronically available to the public. 


\title{
Fiber optic SERS probes for remote sensing
}

\author{
Aditya Pandya \\ Doctor of Philosophy \\ Physics Department \\ Ryerson University
}

2019

\begin{abstract}
Surface Enhanced Raman Spectroscopy (SERS) enhances spontaneous Raman spectroscopy by the virtue of plasmon resonance of nanoparticles. Clinical application of SERS is challenging as nanoparticles remain in the body for long periods of time and a full toxicity analysis has yet to be extensively studied.
\end{abstract}

In this study, Nanosphere lithography (NSL) was used to create optical fibers with nanoparticle enhanced tips for remote sensing using SERS. A custom designed RS collection setup was created for optimal collection of spectra from the optical fibers. It was found that an optical fiber with 0.5 numerical aperture (NA) allowed for better detection of Raman peaks while mitigating the fluorescence background of the optical fiber without any optical filters. Such a sensing platform can potentially be used to temporarily introduce nanoparticles into a sensing environment as it allows retracting the nanoparticles along with the tip.

Nanoporous SERS platform has been fabricated using nanoporous silica glass with $7 \mathrm{~nm}$ and 17 nm pore diameters. An inexpensive fabrication approach of sputter deposition of Au layers was employed on prefabricated nanoporous silica glasses. $7 \mathrm{~nm}$ pore glasses provided larger enhancement than the glasses with $17 \mathrm{~nm}$ pores. A gold layer thickness of $25 \mathrm{~nm}$ was observed to produce largest enhancements. Nanoporous SERS substrates allow a larger effective SERS area 
compared to NSL based fabrication substrates and such nanoporous structures can be potentially fabricated on optical fiber tips for remote sensing.

Finite Element Modeling (FEM) method was implemented for simulating single nanoparticles, an infinite periodic array of nanoparticles and nanoporous films using COMSOL Multiphysics software package. The extinction spectra obtained theoretically were found to match the experimental results for single nanoparticles. The maximum enhancement for the periodic array was two orders of magnitude larger than single particles while the integrated (average) enhancement was only two and a half times larger. Nanoporous films were also modelled using the FEM technique.

Preliminary clinical data were collected from excised breast tissues for evaluating RS as a tool for cancer diagnostics. Spectral peaks from healthy tissues were found to be prominent than cancerous tissues and further experiments are needed to create a multivariate classification model for diagnostics. 


\section{Acknowledgments}

To my parents, Hemant and Ashreeta who patiently supported me through this journey, I am very grateful to you and I owe it all to you. I also thank my very special grandmother, Ansoya aka "women of steel" who despite of all her hardships, loved and cared for me, I dedicate this thesis to you. She taught me no matter how tough life gets, always be positive, loving and caring.

I also would like to thank my supervisors Dr. Alexandre Douplik and Dr. Carl Kumaradas for kindly accepting me as a student and for providing me guidance. It was a perfectly balanced combination of expertise that immensely complemented my thirst for knowledge. Brainstorming sessions with Dr. Douplik were an unforgettable experience and were always fruitful. Dr. Kumaradas' attention to detail, sometimes painful, was hugely beneficial to my work and me personally, I learned a lot from both of you for which I will be eternally grateful. I thank you for all the opportunities you have provided me, and I always felt very comfortable under your tutelage.

I would like to thank Tornado Spectral Systems who lent us their award winning HTVS spectrometer without which this work would not have been possible. Special thanks to Dr. Andy Boorn, Richard Denis, Dr. Bradford Behr, and Sharon Deram for their support.

I am indebted to Dr. Irina Schelkanova for the in-depth discussions and uncountable times she supported me along the way. Very special thanks go to all my dear friends who have provided great moral and emotional support. Thank you Sonal Bhadane, Dr. Homa Asssadi, Victoria Bulycheva, Dr. Sathiyamoorthy Krishnan, Kenneth Webster, Dr. Kenneth Blahut, Dena Monjazebi, Dr. Guennadi Saiko, Harshad Karia and all my friends from India.

A lot would not have been possible without the exceptional support of our department staff. I am very happy that I have had the pleasure working with all of you. Special gratitude goes to Teresa Lee, Leslie Mutic, Cynthia Dy, Adriana Gaertner, Sophia Finos, Maria Teresa Sy, Graham Pearson, Arthur Worthington, Luke Yaraskavitch, Matthew Forrest, Kevin Liu and Graham Ferrier. I would also like to specially thank Sally Noce for the awesome conversations and tennis sessions. I will never forget the life lessons I learned from Iosif Deac, through jokes and anecdotes, and I thank him for always being a source of joy when things were difficult.

I would like to thank all the sources of Funding that I received, OGS, NSERC grants, OCE grants, Learning and Teaching grants, Travel grants (ICRF, Ryerson). I gratefully acknowledge support 
from Dr. Rafi Karshafian, Dr. Ana Milic, Dr. Vladislav Toronov and Dr. Yuan Xu. I am thankful to our collaborators Dr. Csilla Gergely, Dr. Hamideh Salehi, Dr. Marta Martin, France for their support and I would also like to thank our other collaborators Prof. Dr. Jürgen Popp, Prof. Dr. Daniel Razansky from Germany for their guidance and support. I am also very grateful to our collaborator Dr. Olga Andreeva from Russia for providing nanoporous substrates. 


\section{Table of Contents}

Author's declaration for electronic submission of a dissertation .............................................. ii

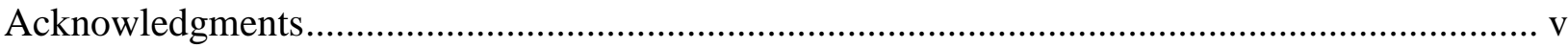

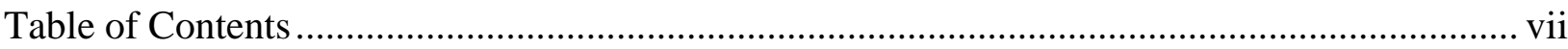

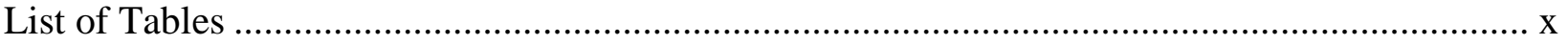

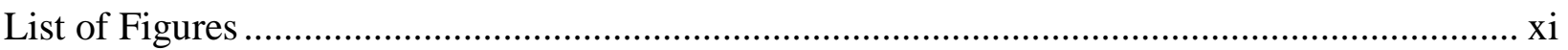

List of Appendices .............................................................................................................

List of Abbreviations ...................................................................................................... xviii

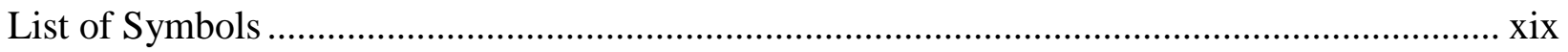

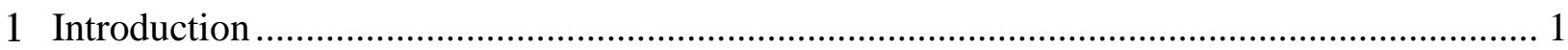

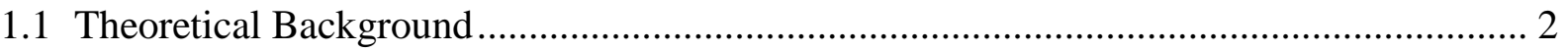

1.1.1 The Physics of Raman spectroscopy ................................................................ 2

1.1.2 Surface Enhanced Raman spectroscopy (SERS) ........................................... 4

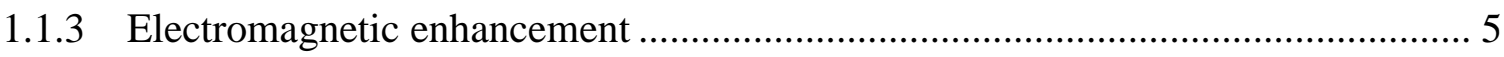

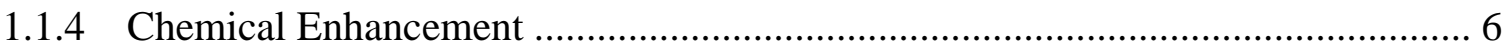

1.2 Raman spectroscopy instrumentation and diagnostic applications ............................. 7

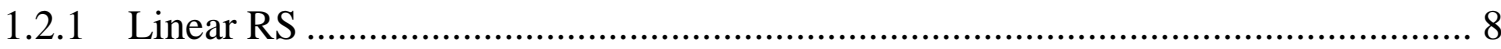

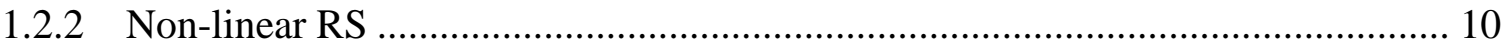

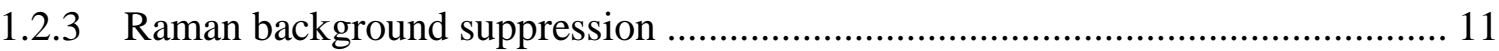

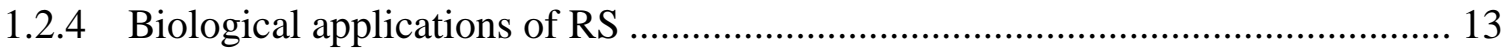

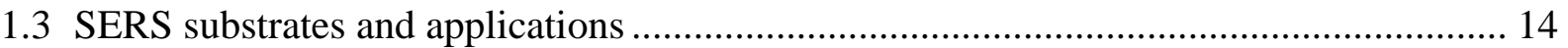

1.3.1 Colloidal SERS substrates ....................................................................... 14

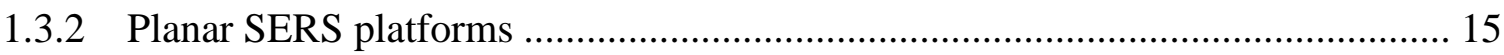

1.3.3 Nanoroughened/Nanoporous SERS substrates ........................................ 17

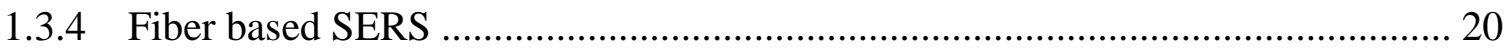

1.3.5 Differences in SERS spectra and Raman spectra ....................................... 21 
1.4 Theoretical Modeling of SERS ............................................................................... 22

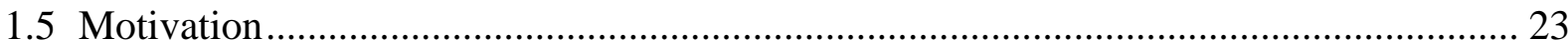

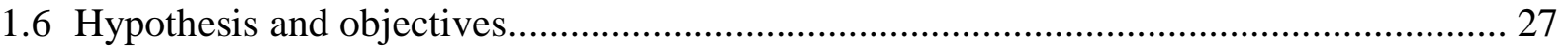

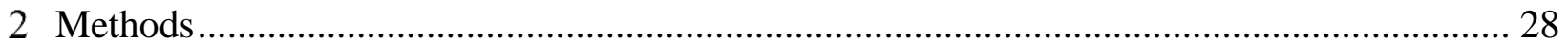

2.1 Instrumentation for Raman spectroscopy ….................................................... 28

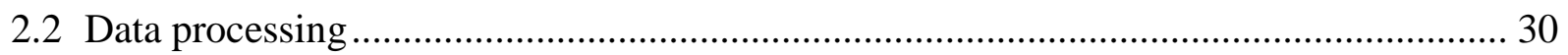

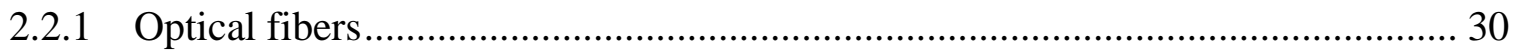

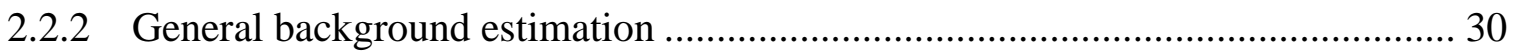

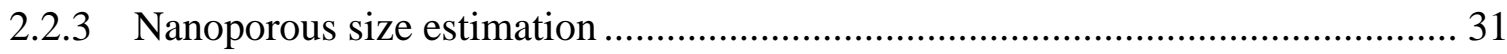

2.2.4 Area under the curve for spectral peaks.................................................. 32

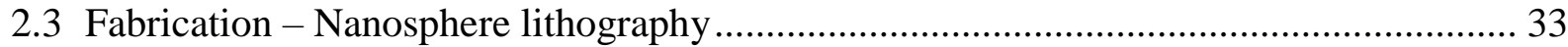

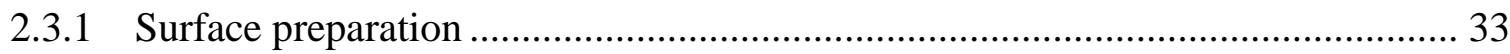

2.3.2 Nanosphere lithography on flat glass substrates ........................................ 35

2.3.3 Nanosphere lithography on optical fiber substrates ..................................... 36

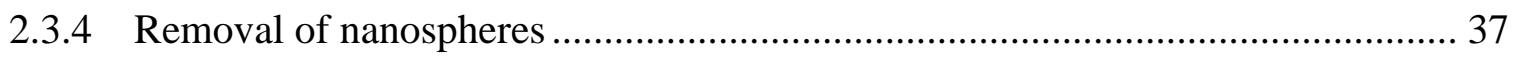

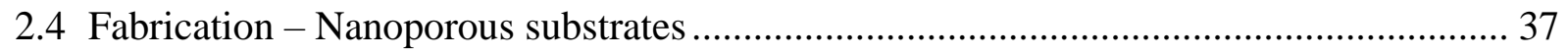

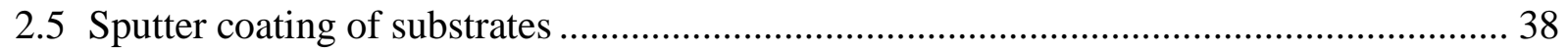

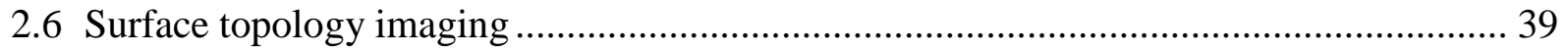

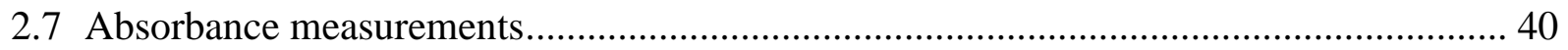

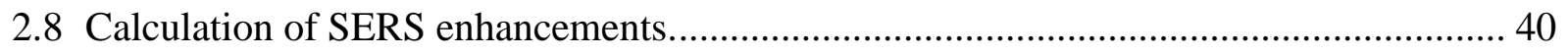

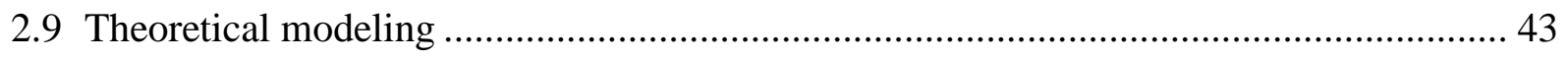

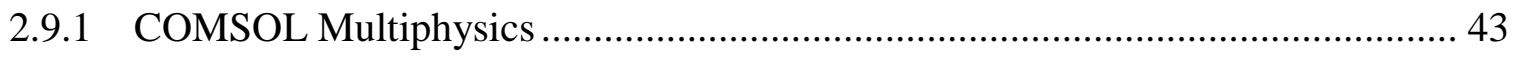

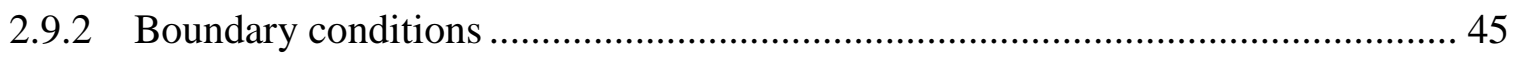

2.9.3 Scattering, absorption and extinction cross sections................................... 47

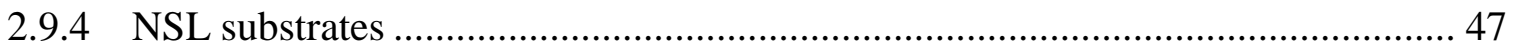

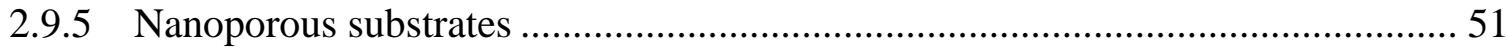




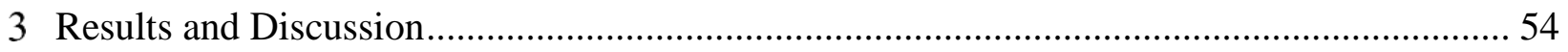

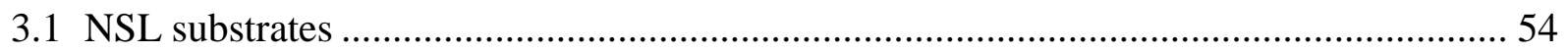

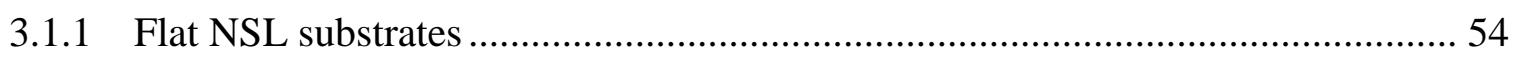

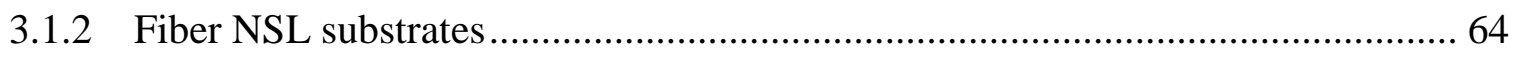

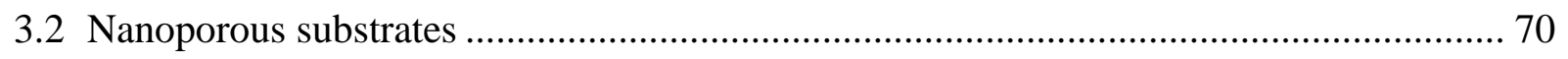

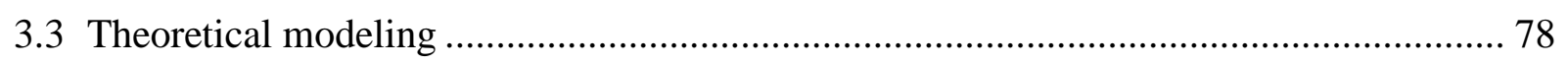

3.3.1 NSL - Single particle simulations ………………………………………..... 78

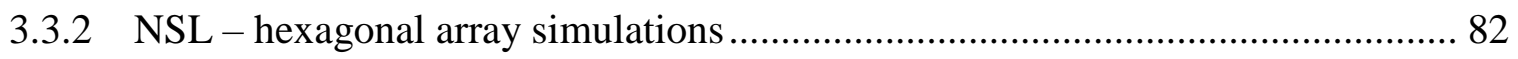

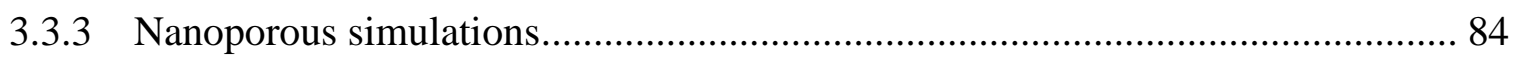

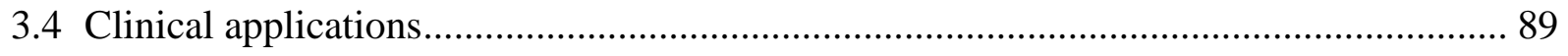

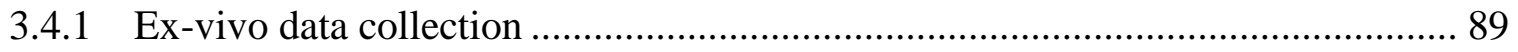

3.4.2 Comparison of data collection from ex-vivo and processed tissues ..................... 92

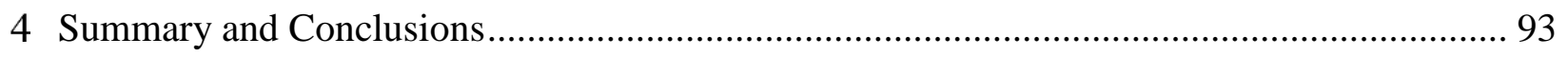

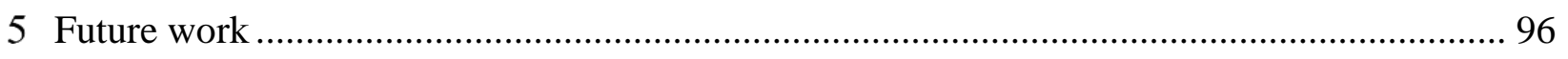

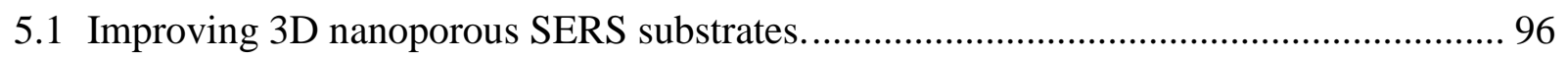

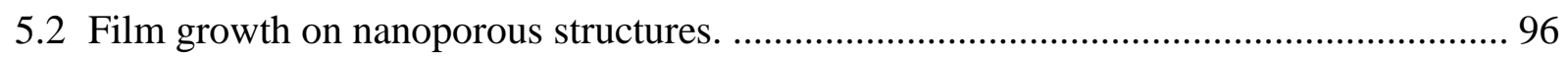

5.3 Nanoporous fiber-optic SERS substrates..................................................................... 96

5.4 Sapphire based fiber optic SERS substrates …………………….................................. 97

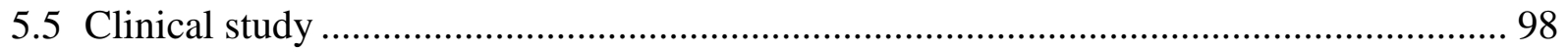

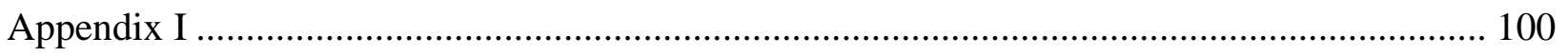

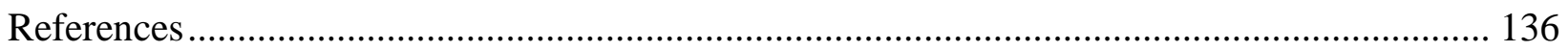




\section{List of Tables}

Table 1-1 Overview of common fabrication methods adapted from [77] ................................ 16

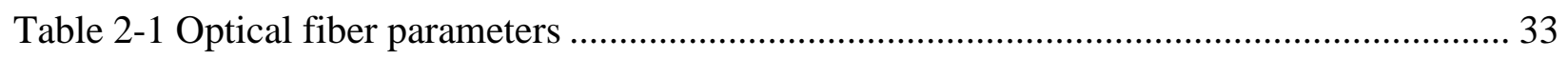

Table 2-2 Characteristics of Nanoporous glasses .......................................................... 38

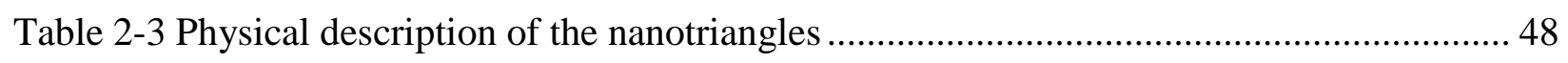

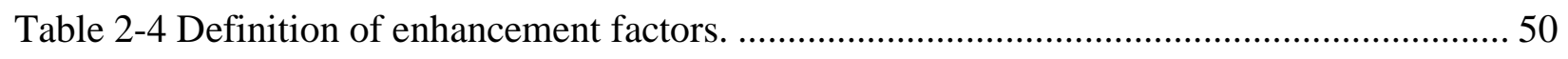

Table 3-1 Concentration tested for NSL SERS substrates................................................... 61 


\section{List of Figures}

Figure 1-1 Comparison of linear (Raman) and non-linear (Resonant Raman (rR), Stimulated Raman Spectroscopy (SRS) and Coherent Anti-Stokes Raman Spectroscopy (CARS) processes for Raman spectroscopy 10

Figure 1-2 Time domain separation of Raman and Fluorescence photons.

Figure 1-3 Frequency domain separation of Raman and Fluorescence photons (adapted from [61].)

Figure 1-4 A) SEM image of nanoporous alumina substrate with $200 \mathrm{~nm}$ average pore size. B) SEM image of nanoporous polycarbonate substrate with $100 \mathrm{~nm}$ average pore size. C) AFM image of nanoporous Silica glass with $17 \mathrm{~nm}$ average pore size. D) SEM image Nanorough Silica glass fabricated using pulsed lasers(adapted from [92]). E) Cellulose membrane substrate 19

Figure 1-5 Various types of fiber-optic SERS sensing strategies. 21

Figure 1-6 Comparison of spectra obtained using absorption, fluorescence and Raman spectroscopies. RS contains distinct peaks and features that are suitable for chemometric analysis

Figure 2-1 Using a $105 \mu \mathrm{m}$ optical fiber (OF1), laser light is collimated using lens (L1) and filtered using a band pass laser line filter (LF). The collimated laser light passes through a dichroic mirror (DIC) which reflects the laser lights on sample. Red shifted wavelengths passed through the DIC and another lens (L2) focused light into a $1 \mathrm{~mm}$ optical fiber which is connected to a Raman spectrometer. A) The laser was focused on the sample (flat substrates, biological tissues) to collect RS and SERS spectra B) The SERS optical fiber (OF3) is excited in the direct and remote configurations to collect SERS spectra. C) Description of optical elements used in the setups. . 29

Figure 2-2 Background suppression method used for optical fibers. The inset shows an expanded view of the Raman shift range $1250 \mathrm{~cm}^{-1}$ to $1650 \mathrm{~cm}^{-1}$ to display the background and SERS spectra. 
Figure 2-3 Background suppression method used for general background suppression. The top plot represents the raw SERS spectra in blue and estimated background in red. The bottom plot represents background corrected spectra.

Figure 2-4 A) Central line profile overlaid on the image B) Radial line profiles extracted for estimating pore size $\mathrm{C}$ ) Line profile corresponding to the central line in A. 32

Figure 2-5 Schematic for computing area under the curve using a linear tangent connecting the end points of the peak.

Figure 2-6 The Process of creating a flat cleaved fiber tip: 1) An optical fiber is cut using scissors and the buffer is removed. The surface of the optical fiber is jagged (depicted by the before image). 2) The fiber is inserted into the bare fiber adapter with $1 \mathrm{~cm}$ of fiber tip with no buffer protruding from the adapter. 3) A fiber cleaver is used with the flat bed of the blade resting on the face of the bare fiber adapter to create a score on the fiber perpendicular to its central axis. 4) The fiber is mechanically pulled to create a flat cleave on the fiber tip (depicted by the after image)..... 34

Figure 2-7 A) Drop coating method of NSL fabrication, and B) Air-water interface based NSL fabrication method for flat substrates. The PS spheres formed a monolayer at the air-water interface and the PS spheres anchored onto the glass slide. The slide was slowly pulled out of water to transfer the monolayer onto the surface of the glass..... 36

Figure 2-8 The air-water interface based NSL fabrication method for optical fiber tips. PS monolayer was formed at the air-water interface. The optical fibers were pulled in the upward direction and the monolayer was transferred onto the tip of optical fibers. 37

Figure 2-9 Sputter coating of flat glass substrates and optical fiber substrates.

Figure 2-10 Complex refractive index (n-real part, k-imaginary part, unitless) of gold used in the study. 46

Figure 2-11 A) SEM image of a monolayer of PS spheres B) A boolean difference operation is performed on a flat rectangle to create a curved nanotriangle. C) The flat sketch is extruded to appropriate heights to create a $3 \mathrm{D}$ nanotriangle and filet operation is performed on the edges to create smooth surfaces. 48 
Figure 2-12 Schematic of computational domain created for calculating the electromagnetic enhancement of nanotriangles. 1) Periodic boundary conditions (PBC) are applied in the X and Y (hidden in this view) for computing background electric field. 2) Perfectly matched layer (PML) boundaries are used to truncate the domain in each direction. 49

Figure 2-13 Hexagonal periodic arrangement of the nanotriangles with insets showing unit cell.

Figure 2-14 Computational setup in for periodic simulation of the hexagonal periodic cell. ...... 51

Figure 2-15 3D model of nanoporous film and computational domain for modeling nanoporous structures. The top row shows the binarization of the topographical image. The edge elements are truncated due to the field of view and are omitted from the 3D modeling. 53

Figure 3-1 Results of the drop coating technique. 1) Optical image of the drop coat showing central monolayer and thicker edges showing a larger amount of PS spheres. 2) SEM images of low and high magnifications of the monolayer. 3) AFM image of the corresponding nanopattern formed after removal of spheres. 55

Figure 3-2 Fabrication process for the air-water interface method 1) The setup depicting the dipper assembly used to lower the glass slides in the Teflon tank. 2) A photographic image of the iridescence observed due to the monolayer on the surface of glass. 3) SEM image of the resultant monolayer over a large surface area and 4) AFM image inset of the resultant nanopattern obtained overlaid on top of the magnified SEM image in the center. 56

Figure 3-3 Heights of nanoparticles obtained from AFM for 1min, 2min and 3min sputter coating of Au. Vertical lines in the height distributions represent $50^{\text {th }}$ percentile and $99^{\text {th }}$ percentile of heights and the values represent the mean value of heights over $50^{\text {th }}$ percentile. 57

Figure 3-4 Spectra comparison for spontaneous Raman and SERS collection modes for 100PPM CV dye. 58

Figure 3-5 Comparison of SERS spectra from patterned and nonpatterned substrates. 59

Figure 3-6 A) Background corrected SERS spectra for 1min, 2min and 3min sputter coats. The blue bump located at $1300 \mathrm{~cm}^{-1}-1400 \mathrm{~cm}^{-1}$ region for $0 \mathrm{~min}$ is artificial due to the fluorescence 
background of the glass and the background suppression algorithm. B) Comparison of integrated intensity for the $1177 \mathrm{~cm}^{-1}$ peak for different sputter times. This peak was chosen due to its prominence and isolation from the surrounding peaks.

Figure 3-7 SERS spectra for NSL substrates plotted in the descending order of concentration from top to bottom (From NSL-100 - NSL-0.1). The vertical lines represent the peak examined to compute the AUC for comparing the intensities. The larger broad peak in the region of $1250 \mathrm{~cm}-$ 1 to $1550 \mathrm{~cm}-1$ is due to the fluorescence of the glass substrate.

Figure 3-8 Concentration dependence of NSL SERS substrates and variation in fabricated batches.

Figure 3-9 Intra batch spectra for NSL - 100 substrates. 63

Figure 3-10 Absorption spectra of identically fabricated flat NSL substrates. Spectra from 5 identical substrates are presented. 64

Figure 3-11 NSL fabrication process for optical fiber tips. Brightfield microscopic images of the prepared optical fiber tips are presented in the first column. The second column depicts tips coated with a monolayer of PS microspheres. The third column represents a binary image with thresholds applied to evaluate the coating area of the optical fibers. 65

Figure 3-12 A) Fiber-optic tips after the NSL fabrication process B) AFM image showing the resultant nanopattern on the fiber tip C) Fibe-optic tip before NSL fabrication D) SEM image of the monolayer of PS spheres on the fiber-optic tip. 66

Figure 3-13 The autofluorescence background generated by the optical fibers used in the study.

Figure 3-14 The spectra are arranged in decreasing order of NA from top to bottom and are normalized for visualization purposes. The spectra are offset equally, and the topmost spectrum was acquired using a flat substrate fabricated on a microscopic cover slip. The vertical dashed lines in the remote configuration mark the peak $\left(1620 \mathrm{~cm}^{-1}\right)$ that was used to compare the peak intensity for different NA. The spectra are normalized (the scales of each subplot separated by dashed horizontal lines is between 0 and 1) for better depiction of the peaks. Although the spectra were 
corrected for background, some of the resultant artefacts related to the strong background were still present in the processed spectra.

Figure 3-15 Integrated peak corresponding to the dashed lines in Figure 3-14 with a fit to NA ${ }^{2}$. The vertical lines for each point represent one standard deviation. 70

Figure 3-16 AFM images of NS-17 and NS-7 substrates, the AFM scan area was $800 \mathrm{~nm} \times 800 \mathrm{~nm}$.

Figure 3-17 Pore size distribution for NS-7 and NS-17 substrates. 72

Figure 3-18 AFM images depicting the surface of NS-7 and NS-17 substrates after sputter coating of Au layers. 73

Figure 3-19 Pore size distribution for Au coated NS-7 and NS-17 substrates. 74

Figure 3-20 Variation of pore sizes with Au layer thickness for NS-7 and NS-17 substrates depending on coating thickness. The slope is larger for the NS-7 substrates indicating that the pores filled up faster. 75

Figure 3-21 SERS spectra for NS-7 and NS-17 substrates with varying Au layer thicknesses. .. 76

Figure 3-22 A) Dependence of SERS intensities on thickness of Au coating. B) Concentration

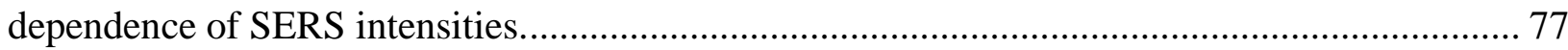

Figure 3-23 enslcompfor single nanotriangles for the wavelength range $500 \mathrm{~nm}-880 \mathrm{~nm}$. The wave propagation direction and the polarization of the electric field is depicted on the figure... 78

Figure 3-24 Extinction spectra obtained from simulations compared with experimental data. ... 79

Figure 3-25 Dependence of extinction spectra on isotropic scaling of nanoparticle size............ 80

Figure 3-26 Dependence of extinction spectra on the height of the nanoparticle. .................... 81

Figure 3-27 Dependence of enhancement factors on the height of the nanoparticle ................. 82

Figure 3-28 enslcompfor an infinite array of periodic nanoparticles for wavelength range 500 $\mathrm{nm}-880 \mathrm{~nm}$. 
Figure 3-29 Integrated and maximum electric field enhancement factors for single particle and periodic array simulations. 84

Figure 3-30 enslcompfor NS-7 substrates for different layer thicknesses $(5 \mathrm{~nm}-80 \mathrm{~nm})$. The enhancement increases slightly with layer thickness and then gradually decreases for increased

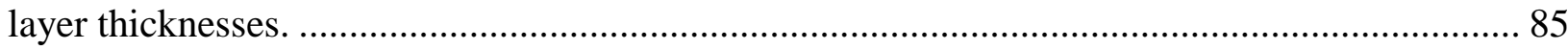

Figure 3-31 enslcompfor NS-17 substrates for different layer thicknesses (5 nm - $80 \mathrm{~nm})$. The enhancement increases with increasing thickness and then start gradually decreasing.............. 86

Figure 3-32 A) The blue shaded region used for volume integration. B) The blue shaded region used for surface integration of NS-7 and NS-17 substrates. 87

Figure 3-33 Integrated E field (Eporousint) for NS-7 and NS-17 substrates. ....................... 88

Figure 3-34 eporouscomp for NS-7 and NS-17 substrates.......................................... 89

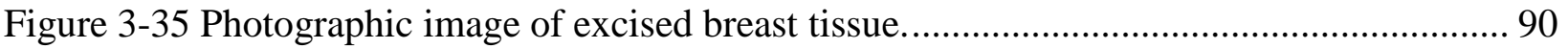

Figure 3-36 Raman spectra collected from excised breast tissues for patients....................... 91

Figure 3-37 Comparison of spectral data collection from ex-vivo and processed tissues. The exvivo data collection was done using a custom Raman setup and a confocal microscope was used

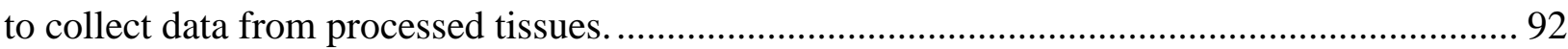

Figure 5-1 Optical fiber with nanoporous tips........................................................... 97

Figure 5-2 Comparison of background generated for silica and sapphire based optical fibers.... 98 


\section{List of Appendices}

Appendix I - Raman spectral library 


\section{List of Abbreviations}

2D

3D

AAO

AFM

Ag

ANSI

$\mathrm{Au}$

AUC

CAD

CARS

CMOS

DDA

DNA

EBL

EM

FDTD

FEM

LDA

LSPR

MPE

NA

NS

NSL

OAD

$\mathrm{OH}$

PCA

PS

RNA

rR

RS

SEM

SERS

SNR

SRS

SVM

UV
Two Dimensional

Three Dimensional

Anodisic Aluminum Oxide

Atomic Force Microscope

Silver

American National Standards Institute

Gold

Area Under the Curve

Computer Aided Design

Coherent Anti-Stokes Raman Spectroscopy

Complementary Metal Oxide Semiconductor

Discrete Dipole Approximation

Deoxyribonucleic acid

Electron Beam Lithography

Electromagnetic

Finite Difference Time Domain

Finite Element Method

Linear Discriminant Analysis

Localized surface plasmon resonance

Maximum Permissible Exposure

Numerical aperture

Nanoporous Silica

Nanosphere Lithography

Oblique Angle Deposition

Hydroxyl ion

Principal Component Analysis

Polystyrene

Ribonucleic acid

Resonant Raman spectroscopy

Raman Spectroscopy

Scanning Electron Microscope

Surface Enhanced Raman Spectroscopy

Signal to Noise Ratio

Stimulated Raman spectroscopy

Support Vector Machines

Ultraviolet 


\section{List of Symbols}

\begin{tabular}{|c|c|}
\hline$q_{k}$ & The $k^{t h}$ normal coordinate for the $k^{t h}$ vibration mode of a molecule \\
\hline$q_{k 0}$ & The equilibrium position for a vibration mode \\
\hline$\omega_{\text {vib }}$ & Frequency of a vibration mode $\left(\mathrm{rad} \cdot \mathrm{s}^{-1}\right)$ \\
\hline$\omega_{0}$ & Frequency of incident electric field $\left(\mathrm{rad} \cdot \mathrm{s}^{-1}\right)$ \\
\hline$\omega_{\text {exc }}$ & Frequency of exciting electric field (analogous to $\left.\omega_{0}\right)\left(\mathrm{rad} \cdot \mathrm{s}^{-1}\right)$ \\
\hline$\omega_{s c a}$ & Frequency of Raman scattered electric field $\left(\mathrm{rad} \cdot \mathrm{s}^{-1}\right)$ \\
\hline $\mathrm{t}$ & Time (s) \\
\hline $\boldsymbol{E}$ & Electric field $\left(\mathrm{V} \cdot \mathrm{m}^{-1}\right)$ \\
\hline$\mu^{\prime}$ & Induced dipole moment $(\mathrm{C} \cdot \mathrm{m})$ \\
\hline$\alpha$ & Polarizability tensor \\
\hline$\alpha_{i j}$ & Elements of the polarizability tensor $\left(\mathrm{C} \cdot \mathrm{V}^{-1} \cdot \mathrm{m}^{2}\right)$ \\
\hline$\beta$ & Hyperpolarizabillity $\left(\mathrm{C} \cdot \mathrm{V}^{-2} \cdot \mathrm{m}^{3}\right)$ \\
\hline$\gamma$ & Second hyperpolarizability $\left(\mathrm{C} \cdot \mathrm{V}^{-3} \cdot \mathrm{m}^{4}\right)$ \\
\hline$\varepsilon(\omega)$ & Dielectric permittivity as a function of frequency $\left(\mathrm{F} \cdot \mathrm{m}^{-1}\right)$ \\
\hline$\eta(\omega)$ & Refractive index (1) \\
\hline$\lambda$ & Wavelength (nm) \\
\hline$E_{\text {surf }}$ & Local electric field close to the surface of the nanoparticle $\left(\mathrm{V} \cdot \mathrm{m}^{-1}\right)$ \\
\hline$I E F_{\text {surf }}$ & Intensity enhancement factor at the surface of the nanoparticle (1) \\
\hline$I E F_{\text {total }}$ & Overall intensity enhancement factor for SERS (1) \\
\hline$E F_{\mathrm{SERS}}$ & Analytical Enhancement factor for SERS (1) \\
\hline$E F_{\text {SSERS }}$ & Substrate Enhancement factor for SERS (1) \\
\hline$I_{\text {SERS }}$ & Intensity of SERS spectra $\left(\mathrm{W} \cdot \mathrm{cm}^{-2}\right)$ \\
\hline$I_{\mathrm{RS}}$ & Intensity of Raman spectra $\left(\mathrm{W} \cdot \mathrm{cm}^{-2}\right)$ \\
\hline$c_{\text {SERS }}$ & Concentration of analyte used in SERS (M) \\
\hline$c_{\mathrm{RS}}$ & Concentration of analyte used in RS (M) \\
\hline$N_{\text {surf }}$ & Number of molecules on the surface of nanoparticle (1) \\
\hline$N_{\mathrm{vol}}$ & Number of molecules in the collection volume for RS (1) \\
\hline$e_{\mathrm{I}}^{\text {flat }}$ & Intensity enhancement factors for flat substrates (1) \\
\hline I flat & SERS intensity of flat substrates $\left(\mathrm{W} \cdot \mathrm{cm}^{-2}\right)$ \\
\hline I flat & $\mathrm{RS}$ intensity of flat substrates $\left(\mathrm{W} \cdot \mathrm{cm}^{-2}\right)$ \\
\hline$e_{\mathrm{I}}^{\text {fiber }}$ & Intensity enhancement factors for fiber substrates (1) \\
\hline $\begin{array}{l}\text { fiber } \\
\text { fibERS }\end{array}$ & SERS intensity for fiber substrates $\left(\mathrm{W} \cdot \mathrm{cm}^{-2}\right)$ \\
\hline$I_{R S}^{\text {fiber }}$ & $\mathrm{RS}$ intensity for fiber substrates $\left(\mathrm{W} \cdot \mathrm{cm}^{-2}\right)$ \\
\hline D & Electric displacement $\left(\mathrm{C} \cdot \mathrm{m}^{-2}\right)$ \\
\hline$\rho$ & Electric charge density $\left(\mathrm{C} \cdot \mathrm{m}^{-2}\right)$ \\
\hline $\mathbf{H}$ & Magnetic field intensity $\left(\mathrm{A} \cdot \mathrm{m}^{-1}\right)$ \\
\hline B & Magnetic flux density $\left(\mathrm{Wb} \cdot \mathrm{m}^{-2}\right)$ \\
\hline$J$ & Current density $\left(\mathrm{A} \cdot \mathrm{m}^{-2}\right)$ \\
\hline$\epsilon_{0}$ & Dielectric permittivity of free space $\left(\mathrm{F} \cdot \mathrm{m}^{-1}\right)$ \\
\hline$\epsilon_{r}$ & Relative permittivity of the medium (1) \\
\hline$\mu_{0}$ & Permeability of free space $\left(\mathrm{H} \cdot \mathrm{m}^{-1}\right)$ \\
\hline$\mu_{r}$ & Relative permeability of free space (1) \\
\hline$\sigma$ & Conductivity $\left(\mathrm{S} \cdot \mathrm{m}^{-1}\right)$ \\
\hline
\end{tabular}




\section{Introduction}

Biosensors that are efficient, low-cost, non-destructive, and label-free are desirable for identification of bio-markers that are used for clinical diagnosis[1]-[3]. The ability to map the spatial distribution of bio-markers allows for identification of the regions of diseased areas in tissue, which aids in the early detection of disease and their development [4]. Optical spectroscopy and spectroscopic imaging have been examined for this purpose [5]. Recently, there have been many studies reporting the use of spectroscopic techniques towards diagnosing diseased conditions [6]-[9]. The degree of multiplexing (e.g. detecting multiple analytes through a single spectral acquisition) and versatility of spectral techniques in molecular sensing domain has been well established in the literature [4], [6], [8], [10]. In an extensive review performed by Dr. Gambhir, he states: "In the future, more emphasis needs to be placed on developing new molecular imaging strategies whereby imaging agents are not required, yet a large diversity of targets can be interrogated." [4]

One of the most promising spectral techniques for molecular imaging/detection is Raman spectroscopy (RS). RS provides a chemical fingerprint of a sample by utilizing the molecular vibrational information gathered by the inelastic interaction of light with molecules. A frequency shift in the scattered light is caused by energy transfer which is proportional to the energy of a specific vibrational mode of a molecule. RS has the ability to detect individual molecules[11], [12] and the frequency shifts are independent of excitation wavelength. A library of pure form individual RS spectra for chemicals can be used to identify the chemical composition of an unknown sample. Application specific wavelengths can be used for custom experiments while the same data library measured at a fixed wavelength can be used to identify the chemical composition. The most important feature of Raman spectra is that they are sensitive biochemical markers due to the unique vibrational fingerprint spectra of the chemical compounds. Considering that disease pathology is always related to biochemistry, biochemical imaging has become important in medical diagnostics. Although RS has been mainly used in analytical chemistry, it has recently shown potential as a clinical diagnostic tool for discriminating between diseased and normal tissue, using endogenous spectra [4], [13]-[15]. Raman spectroscopy has become a widely used method to characterize biological tissues[6], [16], [17]. RS has been widely used in cancer detection, 
allograft rejection analysis, blood analyte analysis, drug detection and cardiovascular analysis [18]-[20]. Optical biopsy using RS has been used to detect tissue suspected of being cancerous without removal, through collecting RS spectra from a single point or obtaining spatial maps [21].

Despite the benefits, traditional RS was considered limited for diagnostic use because of the poor efficiency of the inelastic scattering processes and thus the relatively weak signal [22]. Surfaceenhanced Raman spectroscopy (SERS) combines molecular specificity with single-molecule sensitivity [23]. A signal enhancement of several orders of magnitude (higher than 10 orders of magnitudes has been reported in the literature) can be achieved. The sensitivity of SERS and its multiplexing capabilities are major strengths of the technique. Also, the fact that Raman signals are detectable over long periods of time (minutes-hours without issues of photo bleaching) means that longitudinal studies are feasible [4]. In this work, enhancements to RS and SERS have been investigated in this thesis for applicability to remote sensing. Such a device is estimated to form the basis of accurate and sensitive remote sensing for uses in opto-fluidic and endoscopic based diagnostics.

\subsection{Theoretical Background}

Raman spectroscopy was named after Sir Chandrasekhara Venkata Raman who established the field of inelastic scattering of light experimentally for which he received the Nobel Prize in Physics in 1930 [24]. The interaction of electromagnetic radiation with matter occurs through the processes: absorption, emission or scattering. Elastic scattering (Rayleigh scattering and/or Mie scattering) is the most prominent interaction followed by the other processes. The inelastic scattering of light (Raman scattering) is one of the least prominent interactions and is useful for probing the molecular vibrations.

\subsubsection{The Physics of Raman spectroscopy}

The simplest model of absorption, emission and scattering of light is an oscillating electric dipole. The separation of the positive and negative charges in molecules results in the formation of an electric dipole. A simple approximation of an oscillating dipole is an elastic spring coupled to two objects. These oscillations are periodic in nature and can be described using time dependent simple harmonic motion as 


$$
q_{k}=q_{k 0} \cos \omega_{\mathrm{vib}} t
$$

where $q_{k}$ is the $k^{t h}$ normal nuclear coordinate for the $k^{\text {th }}$ vibration mode of a molecule, $q_{k 0}$ is the equilibrium position for that normal vibration mode and $\omega_{\text {vib }}$ is the frequency of the vibration.

Scattered light is proportional to the induced dipole moment $\mu^{\prime}(\mathrm{C} \cdot \mathrm{m})$ which is depends on the applied incident field, $\boldsymbol{E}\left(\mathrm{V} \cdot \mathrm{m}^{-1}\right)$, and is given by

$$
\mu^{\prime}=\alpha E+\frac{1}{2} \beta E E+\frac{1}{6} \gamma E E E+\cdots
$$

where $\boldsymbol{\alpha}$ is the polarizability, $\boldsymbol{\beta}$ is the hyperpolarizability and $\boldsymbol{\gamma}$ is the second hyperpolarizability of the molecule, which are tensors of rank 2, 3 and 4 respectively [25]. Polarizability can be envisioned as the degree of flexibility of the electron cloud to be displaced. Since $\|\boldsymbol{\beta}\| \ll\|\boldsymbol{\alpha}\|$ and $\|\boldsymbol{\gamma}\| \ll\|\boldsymbol{\alpha}\|$ the induced polarizability can be given by

$$
\boldsymbol{\mu}^{\prime}=\boldsymbol{\alpha} \boldsymbol{E} .
$$

Generally, this equation is evaluated in matrix form where $\alpha_{i j}\left(\mathrm{C} \cdot \mathrm{V}^{-1} \cdot \mathrm{m}^{2}\right)$ represents the components of the second rank polarizability tensor.

The polarizability tensor may be modulated by the normal vibrations of the molecule. This dependence can be expressed in terms of a Taylor series [25]

$$
\alpha_{i j}=\left(\alpha_{i j}\right)_{0}+\sum_{k}\left(\frac{\delta \alpha_{i j}}{\delta q_{k}}\right)_{0} q_{k}+\frac{1}{2} \sum_{k, l}\left(\frac{\delta^{2} \alpha_{i j}}{\delta q_{k} \delta q_{l}}\right)_{0} q_{k} q_{l}+\cdots
$$

where the subscripts 0 represent values at equilibrium.

To simplify, the double harmonic approximation is used to neglect the electrical and mechanical anharmonicity (terms involving higher powers $(>1)$ of $q$ ) which transforms the above equation

$$
\boldsymbol{\alpha}_{\boldsymbol{k}}=\boldsymbol{\alpha}_{0}+\left(\frac{\delta \boldsymbol{\alpha}_{\mathrm{k}}}{\delta q_{k}}\right)_{0} q_{k}
$$

where $\boldsymbol{\alpha}_{\boldsymbol{k}}$, is the polarizability tensor for the $k^{\text {th }}$ vibration. 
Considering a time varying electric field $\boldsymbol{E}$ with an amplitude of $\boldsymbol{E}_{\mathbf{0}}$ and a frequency of $\omega_{0}$

$$
\boldsymbol{E}=\boldsymbol{E}_{\mathbf{0}} \cos \omega_{0} t
$$

Using equation 1-1 and equation 1-5 in equation 1-3 for $k^{t h}$ vibration and using trigonometric transformation yields

$$
\boldsymbol{\mu}^{\prime}=\boldsymbol{\alpha}_{\mathbf{0}} \boldsymbol{E}_{\mathbf{0}} \cos \omega_{0} t+\frac{1}{2}\left(\frac{\delta \boldsymbol{\alpha}_{k}}{\delta q_{k}}\right)_{0} q_{k 0} \boldsymbol{E}_{\mathbf{0}}\left[\cos \left(\left(\omega_{0}-\omega_{\mathrm{vib}}\right) t\right)+\cos \left(\left(\omega_{0}+\omega_{\mathrm{vib}}\right) t\right)\right]
$$

The above equation shows three terms that reflect Rayleigh scattering $\left(\omega_{0}\right)$, stokes Raman scattering $\left(\omega_{0}-\omega_{\text {vib }}\right)$ and anti-stokes Raman scattering $\left(\omega_{0}+\omega_{\text {vib }}\right)$ which can be observed independently of an incident frequency. It can also be seen that for a normal vibration mode to be Raman active, it must follow that at least one component of the polarizability tensor must be nonzero i.e. $\left(\frac{\delta \boldsymbol{\alpha}_{k}}{\delta q_{k}}\right)_{0} \neq 0$.

\subsubsection{Surface Enhanced Raman spectroscopy (SERS)}

The experimental discovery of SERS occurred when unusually high Raman signals were observed at the surface of silver electrodes during electrochemical experiments [26]. The first SERS experiments were performed in the late 1970s and it expanded the abilities of Raman spectroscopy for a variety of applications due to the enhancements of the signals. The enhanced signal was only observed after topographical surface changes of the smooth silver electrodes due to electrically induced dissolution and redeposition of the electrode's material on its surface[27]. This indicated that roughness of the surface played an important role in the enhancement mechanisms. Two modes of SERS enhancements[28]-[30]: electromagnetic and chemical (charge transfer) have been proposed. To explain SERS, electromagnetic (EM) enhancement mechanism is widely used and it accounts for most of the total enhancement. The overall enhancements experimentally obtained were larger than the EM enhancement pointing towards the chemical mechanism of enhancement. 


\subsubsection{Electromagnetic enhancement}

The dielectric permittivity, $\varepsilon(\omega)\left(\mathrm{F} \cdot \mathrm{m}^{-1}\right)$, is the response function of a material to an external electric field. Along with the material's size and shape it determines the scattering and absorption of the material. The refractive index can be approximated by $\eta(\omega)=\sqrt{\varepsilon_{r}(\omega)}$, where $\varepsilon_{r}(\omega)$ is dimensionless frequency dependent relative permittivity, ( which is given by $\varepsilon_{r}(\omega)=\frac{\varepsilon(\omega)}{\varepsilon_{0}}$ and the vacuum permittivity is a constant $\varepsilon_{0}=8.854 \times 10^{-12} \mathrm{~F} \cdot \mathrm{m}^{-1} \cdot \varepsilon_{r}(\omega)$ and $\eta(\omega)$ are complex quantities given by,

$$
\varepsilon_{r}(\omega)=\varepsilon_{\text {real }} \pm i \varepsilon_{\text {imag }}=(\eta \pm i \kappa)^{2}
$$

where $\varepsilon_{r}$ and $\varepsilon_{i}$ are the real and the imaginary parts of the permittivity, $\eta$ is the refractive index and $\kappa$ is the extinction coefficient.

The collective oscillations of the electrons (plasmons) give rise to the electromagnetic enhancement mechanism of SERS. This occurs on the surface of nanoparticles where plasmon oscillation (surface and volume charge density oscillation of the metal's free electron gas) is sustained due to the external EM field and is termed as localized surface plasmon resonance (LSPR). To compute the LSPR for SERS, we assume a model of a small single nanosphere $\varepsilon(\omega)$ with diameter $a \ll \lambda$ embedded in a dielectric medium with the relative permittivity of $\varepsilon_{m}(\omega)$ under the action of a time varying, spatially static uniform electric field $E_{0} e^{-i \omega t}$ polarized along the $z$-axis [31]. The electrostatic approximation of Maxwell's equation can be used given the size constraints [32]. Hence, for static charges, the Maxwell's equations can be replaced with Laplace's equation for scalar electric potential $\nabla^{2} \Phi=0$ to calculate potential inside and outside the nanosphere. At $r \rightarrow \infty$, electric field outside the sphere is equal to the applied external electric field $\boldsymbol{E}_{\text {out }}=-E_{0} \hat{\boldsymbol{z}}$. Using these conditions, solving for the potential outside the sphere to obtain the electric field $(\boldsymbol{E}=-\nabla \Phi)$ surrounding the particle is given by:

$$
\boldsymbol{E}_{\text {out }}(x, y, z)=-E_{0} \hat{\boldsymbol{z}}+\left[\frac{\varepsilon(\omega)-\varepsilon_{m}(\omega)}{\varepsilon(\omega)+2 \varepsilon_{m}(\omega)}\right] a^{3} E_{0}\left[\frac{\hat{\boldsymbol{z}}}{r^{3}}-\frac{3 z}{r^{5}}(x \widehat{\boldsymbol{x}}+y \widehat{\boldsymbol{y}}+z \hat{\mathbf{z}})\right]
$$

where an enhancement factor $g_{m}$ can be defined as: 


$$
g_{m}=\left[\frac{\varepsilon(\omega)-\varepsilon_{m}(\omega)}{\varepsilon(\omega)+2 \varepsilon_{m}(\omega)}\right]
$$

For full derivation of the above equation please refer to the supplementary material provided by Willets et.al [31]. The first term of the equation 1-9 corresponds to the applied electric field and the second term corresponds to the induced dipole resulting from the polarization. $g_{m}$ is the enhancement factor for the electric field which is responsible for the electromagnetic enhancement of SERS. The above enhancement factor is suitable for a nanosphere only under the small size approximations but provides an insight on the basic mechanism of electromagnetic enhancement. Complex shaped nanoparticles can be difficult to estimate analytically, and numerical calculations

are required for such cases. Due to the $\frac{1}{r^{3}}$ dependence of the electric field outside the nanosphere, it is evident that the LSPR is a near field effect. From equation 1-10, it can be noticed that the maximum enhancement occurs for when $\varepsilon(\omega) \rightarrow 2 \varepsilon_{m}$ which describes the resonant condition for an isotropic sphere. For noble metals, the size and shape of the nanoparticles permit this resonance condition to occur at visible frequencies allowing for a wide range of applicability. The enhancement depends on the shape of the nanoparticle and further analytical solutions for different shapes (for e.g. cylinders) revealed the LSPR is strongly dependent on the shape of the nanoparticle [33]. The "lightning rod effect" (concentration of dipole fields at sharp edges) is responsible for immensely large enhancements at sharp edges of nanoparticles [34]. This gives rise to hot-spots that allow probing of molecules at low concentrations down to single molecules[35], [36].

\subsubsection{Chemical Enhancement}

Apart from the plasmonic enhancement of nanoparticles, molecules can also chemically or electronically interact with the metal. Several different modes of chemical enhancements have been proposed. The adsorption of molecules onto the nanoparticles results in molecularnanoparticle complexes which can alter the polarizability, resulting in larger Raman cross sections of the metal-molecule complex. This is termed "static charge transfer"[37]. It is also possible that there can be temporary electron/hole transfers between the metal and molecules which are termed "transient charge transfer" [37] that alter the vibrational modes of the molecule. Due to the charge transfer mechanisms, new intermediate electronic states can be formed resulting in new resonance Raman processes that are not present for individual molecules in absence of nanoparticles[37]. 
Only a small fraction of the molecules were found to exhibit chemical enhancement experimentally [38] indicating specific sites for chemical/electronic interactions.

Due to the multimodal nature of SERS enhancements, there have been various studies that have tried to provide a unified approach towards modeling SERS which include combining the charge transfer mechanisms, molecular resonance and plasmon resonance [39]-[41]. Although the theoretical foundations have been laid for a unified expression of SERS, extensive experiments need to be performed for proper evaluation of the relative contribution of each of these enhancement mechanisms.

\subsection{Raman spectroscopy instrumentation and diagnostic applications}

$\mathrm{RS}$ is advantageous for the histochemical analysis of biological tissues in comparison to other spectroscopic methods. In endoscopic imaging, RS can be used to qualitatively and quantitively study the biochemical constituents of an area being investigated. This may involve diagnosis of diseases of the soft tissues, lens, cornea, blood, hard tissues, arterial disease etc. RS is sensitive to molecular changes that are associated with cancer, such as an increased nucleus-to-cytoplasm ratio, disordered chromatin, higher metabolic activity, and changes in lipid and protein levels [42]. Not only is differentiation between normal and diseased cells possible, but also different chemical states (changes in chemical composition indicating pre-diseased conditions) of organs and cells can be probed [16], [43]. Even complex and heterogeneous white and grey matter of the brain can be easily differentiated with micrometer resolution [44]. Since RS can detect the variations in DNA/RNA, proteins, and lipids, this makes RS an excellent tool for analyzing changes on the cellular level [45]. Another advantage of RS is that the collection of spectra can be performed in vitro, ex vivo or in vivo without disrupting the cellular environment in contrast to other techniques that utilize chemical biomarkers and create nonnative environments for biological tissues [45]. Machine learning using supervised techniques can provide necessary real-time immunohistochemical information which aids in the discrimination of normal and cancerous tissues [18]. Furthermore, RS can identify tumor margins even with a background of stain chemicals present in the samples. RS can be broadly classified into linear and non-linear RS categories and can perform label free sensing. 


\subsubsection{Linear RS}

Linear RS primarily involves the interaction of single frequency laser sources to detect inelastically scattered light. This interaction is generally weak and thus require long acquisition times (in the order of seconds). Typically, around one in $10^{6}-10^{8}$ photons inelastically scatter and contribute to the Raman signal. There are various approaches for linear RS, such as standard RS, confocal RS, low-wavenumber RS, fiber optic RS, and spatially offset RS.

\subsubsection{Standard Raman spectroscopy}

A continuous wave illumination laser source, typically a diode with a narrow linewidth, is used to detect the Stokes shifted RS signal. This is the most widely used approach and has been miniaturized to be used as hand-held devices [46]. RS instrumentation requires a laser with coupling optics, a spectrometer with coupling optics, a dichroic beamsplitter to separate excited and scattered photons and a high numerical aperture (NA) objective for focusing and collection of light. The primary collection mode of instrumentation involves a backscattering collection geometry with co-aligned excitation and scattered pathways. Usually, the signal collected is stokes Raman that is more probable than anti-stokes at room temperature. The stokes Raman photon loses energy with respect to the excitation photon while the anti-stokes Raman gains energy. The stokes Raman signal usually has a lot of interference from other interactions such as fluorescence. Collecting RS from biological media can be challenging since biological tissue has fluorescence that routinely interferes with RS signals.

\subsubsection{Confocal Raman spectroscopy}

The spatial resolution of spontaneous Raman spectroscopy is limited by the optics used. In routine spectroscopic analysis, a point spectrum is collected from a region of interest. This spot size of collection varies from tens of microns to a few mms. For cellular diagnostic applications where, routine imaging needs to be performed, spot spectroscopy is not suitable. Hence, confocal imaging is performed by scanning multiple points in an area. Building upon the standard RS instrumentation, spatial filters (e.g. pinholes, single mode fibers) are used for the laser excitation as well as the spectrometer collection pathways to obtain diffraction limited performance. Laser is employed with single mode optical fibers/spatial filters to obtain a gaussian beam that can be focused onto a diffraction limited spot size. The scattered RS signal is then collected via a 
spectrometer through a complementary spatial filter to match the optical characteristics of the excitation spot [47]. This methodology allows for high resolution spatial mapping of RS signals that can be used to perform chemometric analysis towards label free sensing [48].

\subsubsection{Low wavenumber Raman spectroscopy}

Standard Raman instrumentation record wavenumbers (Raman shifts) in the range of $100 \mathrm{~cm}^{-1}-$ $4000 \mathrm{~cm}^{-1}$. This provides the unique fingerprint of the molecular vibrational structure being examined. This range works for a wide variety of applications but is not suitable for special cases which deal with molecular polymorphism and fine crystalline structure variations [49]. For this, wavenumbers $<100 \mathrm{~cm}^{-1}$ can elucidate features specific to such variations. The instrumentation for low wavenumber RS is different from standard RS where the filtering of Raman scattered light is performed via special filters that have a rapid transition between the blocking and the transmission bands that allow for signals closer to the laser line to pass through [50].

\subsubsection{Raman fiber-optic probes}

Optical fibers are versatile and flexible devices capable of light delivery and collection in arbitrary environments. Commonly available optical fibers are made with silica cores and cladding. They work on the principle of total internal reflection and are efficient for guiding light. Optical fibers come in various diameters and contain different types of dopant impurities. When used as light guides for RS, they exhibit large background signals arising from the inherent fluorescent properties of the silica, which necessitates the use of filtering for excitation and collection fibers. Low-OH (low concentration of hydroxyl ion) silica optical fibers are a choice to use in RS as they have lower background fluorescence compared to the high-OH silica fibers. Due to this background, it is quite challenging to use unfiltered optical fibers for RS. Special optical fibers made from sapphire or hollow cores have been proposed as alternatives to filtered probes allowing for RS collection[51], [52]. An extensive review of fiber optic probes for linear and non-linear Raman applications can be found in a review performed by Latka et. al [53].

\subsubsection{Spatially offset fiber-optic probes}

In reflectance spectroscopy a spatial offset is used between the excitation and collection fiber to increase the penetration depth for collection. The same setup has been used in Raman fiber optic 
probes to collect RS from deeper areas. Various geometries can be manufactured which allow for improving the penetration depth. These have been used to collect Raman spectra from material inside sealed containers without opening the seal and for the detection of powders encapsulated in a medium [54]-[56].

\subsubsection{Non-linear RS}

Non-linear RS usually employs two incident fields which interact non-linearly to create enhanced Raman signal. Two major types of non-linear process are widely employed. Stimulated Raman Scattering (SRS) is a technique where a pump beam at frequency $\omega_{p}$ and a probe(or stokes) beam at frequency $\omega_{s}$ are incident on a sample at the same time (mechanism depicted in Figure 1-1). If the molecular vibration frequency matches the difference in frequency of the pump and probe beams, the pump beam experiences a loss and the probe beam experiences a gain in photon energy due to the inelastic scattering process at that vibrational frequency. This gain or loss is detected and it results in rapid scanning of the sample allowing for video rate imaging using SRS [57]. The SRS signal is linearly proportional to the concentration of molecules being probed.

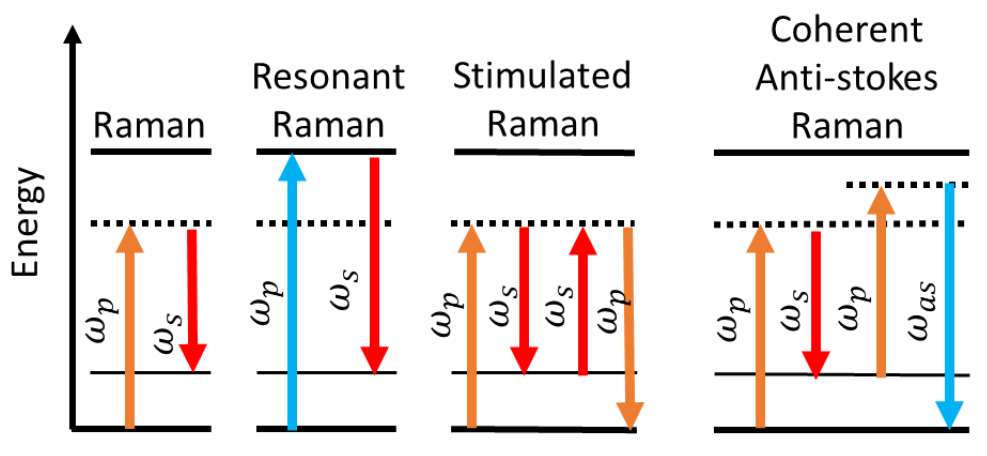

Figure 1-1 Comparison of linear (Raman) and non-linear (Resonant Raman (rR), Stimulated Raman Spectroscopy (SRS) and Coherent Anti-Stokes Raman Spectroscopy (CARS) processes for Raman spectroscopy.

Coherent Anti Stokes Raman scattering (CARS) is a four-wave mixing process that utilizes two incident fields, pump and probe to generate signal at $\omega_{a s}=2 \times \omega_{p}-\omega_{s}$, the anti-stokes frequency. When the frequency difference of pump and probe matches the molecular vibration states, coherent amplification occurs at the anti-stokes frequency. This generates the CARS signal which can provide $\sim 10^{5}$ fold enhancements [58]. CARS allows rapid image scanning and is widely used to perform label-free imaging of excised tissues [59], [60]. Although CARS is quite 
versatile and can be used as an approach to reduce autofluorescence background, it is plagued by a non-resonant background which can decrease the signal to noise ratio significantly and it is expensive to operate and miniaturize.

\subsubsection{Raman background suppression}

The interference from fluorescence can restrict the applicability of RS in samples which exhibit a large fluorescent background. To successfully capture RS from such samples, background suppression needs to be performed to improve the quality of Raman spectra. The technique for background suppression can be divided into experimental methods and computational methods.

\subsubsection{Experimental methods}

\section{Time domain method}

To suppress the background experimentally, time domain spectroscopy and frequency modulation is employed. Time resolved spectroscopy helps to measure RS and fluorescence at the same time while simultaneously allowing to separate RS and fluorescence. RS occurs on the femtosecond scale whereas fluorescence occurs on picosecond - nanosecond scale in the UV to near infrared range. Applying short excitation pulses and resolving the response in time, RS photons can be separated from fluorescence photons to achieve background suppression as shown in Figure 1-2 [61].

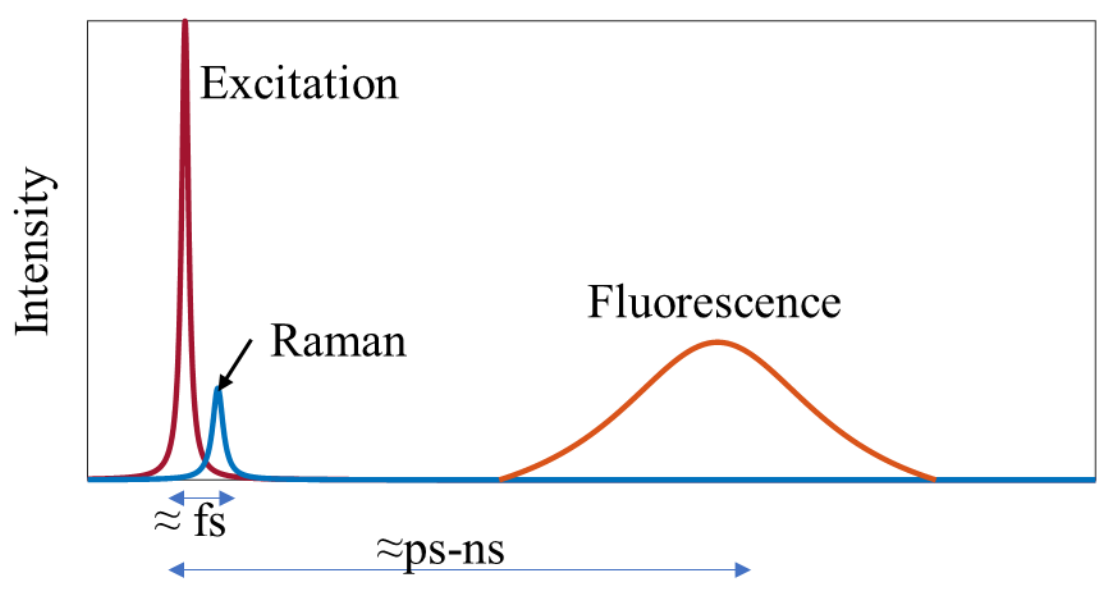

Figure 1-2 Time domain separation of Raman and Fluorescence photons. 


\section{Frequency domain method}

Due to the time difference between RS and fluorescence, frequency modulation methods can also be applied to differentiate RS and fluorescence signals. Applying high frequency modulation excitation, fluorescence signals exhibit an amplitude demodulation and a phase shift with respect to excitation compared to minimal phase shift exhibited by RS signals as shown in Figure 1-3 [61]. Analyzing the phase shift and demodulation, fluorescence can be decreased.

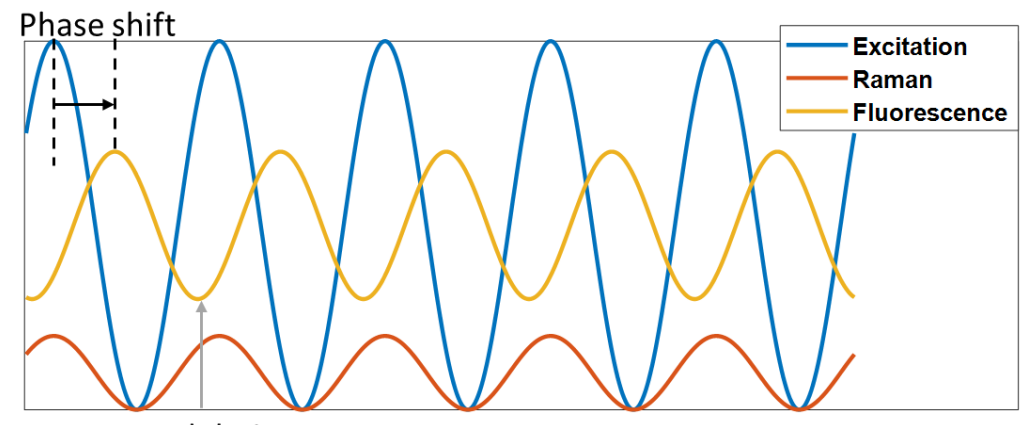

Modulation

Figure 1-3 Frequency domain separation of Raman and Fluorescence photons (adapted from [61].)

\section{Wavelength domain methods}

Another method of background suppression is a wavelength domain technique. Using two slightly shifted excitations of different wavelengths, two spectra are collected where RS peaks are shifted while the fluorescence background stays the same. By a simple subtraction of the shifted spectra, fluorescence suppression can be achieved [62].

\subsubsection{Computational methods}

Computational methods are applied to remove fluorescence background without changing the experimental conditions. A simple $3^{\text {rd }}-4^{\text {th }}$ order polynomial fit can be employed to perform background correction. The order of the polynomial varies according to the experimental conditions (presence of single or multiple fluorophores or other background signals) and when used effectively, it can preserve the shape of the Raman peaks. Another method of background suppression commonly used is to take a derivative of the signal. Since Raman peaks are sharp 
compared to fluorescence peaks, Raman peaks are amplified, and background fluorescence is diminished. The disadvantage of this method is that it can also enhance the noise in the spectrum.

There have been a multitude of other post processing techniques used to do a computational background suppression such as wavelet decomposition, Fourier filtering and principal component analysis which offer varying degrees of background suppression but using derivatives is shown to be a robust method to perform background suppression [61], [63].

\subsubsection{Biological applications of RS}

Raman spectroscopy has been widely employed in clinical trials [18], [43], [45], [64], [65]. During the past 10 years, many studies investigating the use of SERS for ex-vivo studies have also been conducted. Typically, the results from such studies are assessed with sensitivities (percentage of positive samples correctly identified) and specificities (percentage of negative samples correctly rejected). The protocols followed by the studies are similar. Using a gold standard (mostly a biopsy) as a reference, the Raman spectra are collected from diseased areas as well as non-diseased areas. Chemometric analysis using supervised learning is performed using the collected spectra. The most commonly employed multivariate analysis techniques are principal component analysis (PCA), Fisher's linear discriminant analysis (LDA) and support vector machines (SVM) [45]. In an ex-vivo study performed by Mahadevan-Jasen et. al., a comparison of auto fluorescence, diffuse reflectance and Raman spectroscopies was performed for breast tissue discrimination [66]. Using a nonlinear maximum representation and discrimination feature for the feature extraction and sparse multinomial logistic regression for classification, it was reported that the performance of RS was superior to all the other techniques and an overall accuracy of $99 \%$ was achieved. Feld et. al., also reported high accuracies $(>90 \%)$ for breast cancer classification when using a morphological model of basis Raman spectra (pure form spectra of individual components) comprising of cytoplasm, nucleus, fat and other cellular components to fit acquired data with a non-negative least square algorithm in MATLAB ${ }^{\mathrm{TM}}$. The spectra collected from breast tissues were assumed to have a linear weighted sum of the basis spectra for the analysis [67], [68]. Another recent study shows RS being used to monitor the effectiveness of anticancer drugs for chemotherapeutic treatment of cancer patients [69]. Most of these studies were performed in exvivo conditions. 
In a review conducted by Tu et al [18], it was reported that multiple studies performed for cancer diagnosis using RS show that the exposure time ranged from $5 \mathrm{~s}$ to $60 \mathrm{~s}$ and the powers used ranged from $8 \mathrm{~mW}$ to $150 \mathrm{~mW}$ (for different wavelengths). The power reported was of the laser used and not the delivered power to the tissue. These values show that although RS can achieve accurate classification, it lacks in terms of signal acquisition time which is a significant factor for limiting exposure of tissues to laser radiation (higher exposures can potentially result in tissue damage) as well as motion artifacts. The ANSI standard for maximum permissible exposure (MPE) limits the human exposure to laser radiation in the range of $700-1050 \mathrm{~nm}$ is given by MPE $=1.1\left(10^{0.170}\right) t^{0.25}\left(\mathrm{~J} \cdot \mathrm{cm}^{-2}\right)$ where $\mathrm{t}$ is the exposure time in $\mathrm{s}[70]$. Thus, an optimal design for in-situ collection of Raman signals should have a balance between exposure time and intensity. Higher exposure times might require lower intensity decreasing signal to noise ratio (SNR). Thus, decreasing exposure time while limiting intensity is necessary to both improving signal collection as well as making imaging possible.

\subsection{SERS substrates and applications}

After the discovery of SERS from molecules attached to noble metal electrodes, various types of substrates have been prepared to perform SERS. The majority of substrates use Ag or Au since they produce the largest enhancements. SERS substrates can be broadly classified as random or patterned. Random substrates can generate large local enhancement due to randomized sharp features present on the substrate, but they are usually less reproducible. Patterned substrates are more reproducible and produce larger overall enhancements when engineered efficiently but they can be expensive. Usually, SERS substrates are mainly 2D as nanoparticles or nanoroughened surfaces are arranged in a planar manner which ultimately limits the number of molecules contributing to SERS. To remedy this, 3D SERS substrates were created using nanoporous noble metals allowing for probing of a larger number of molecules. This mode of SERS detection varies from using colloidal suspensions with analytes, nanoparticles trapped or fabricated on planar substrates, nanoporous substrates, paper/membrane substrates and optical fiber-based substrates.

\subsubsection{Colloidal SERS substrates}

The most commonly used SERS substrates are colloidal suspensions of nanoparticles mixed with analytes. Various groups have fabricated nanoparticles in colloidal suspensions with a wide variety 
of shapes and sizes. SERS enhancements are usually not reproducible mainly due to inconsistent aggregation of nanoparticles. The easy fabrication of colloidal nanoparticles in different shapes and sizes allows rapid tunability of SERS for a variety of wavelengths and provides a disposable method for single use applications eliminating contamination concerns.

These substrates have also been used in optofluidic/microfluidic platforms that use low sampling volumes and have a controlled geometry which facilitates a sensitive and robust detection platform. Generally, the analytes and nanoparticles are injected into the microfluidic device from different channels, mixed together in the microfluidic through a variety of protocols, and then detected at a specific detection point [71].

\subsubsection{Planar SERS platforms}

Planar SERS substrates (silicon, mica or silica) have been fabricated either by immobilizing nanoparticles on the surface or patterning the substrates through lithography. 2D arrays of nanoparticles with controlled spacing and sizes are required to achieve robust, reproducible and high performance for SERS. A few such methods and their characteristics are presented in Table 1-1. The most ideal method for fabricating uniform and reproducible substrates is the electron beam lithography (EBL) but it is an expensive process when trying to produce large area substrates [72], [73]. The property of self-assembly of nanospheres into monolayers has been utilized to create lithography masks for the process called nanosphere lithography (NSL) [74]. After mask creation, metal is evaporated onto the mask and the nanospheres are removed. This results in a repeating pattern of nanostructures on the substrate formed by the metal filling out gaps between the nanospheres. Template method involves using depositing nanoparticles through tube-like arrays or other 2D templates over the substrate. Another technique which involves deposition of metal particles over nanoporous scaffolds made of silicon or nanorod arrays called the Hybrid method[75]. Oblique angle deposition (OAD) is a method based on conventional vapor deposition technique where deposition occurs at an oblique angles[76]. 
Table 1-1 Overview of common fabrication methods adapted from [77]

\begin{tabular}{cccc|cc}
$\begin{array}{c}\text { Fabrication } \\
\text { method }\end{array}$ & EBL & NSL & $\begin{array}{c}\text { Template } \\
\text { method }\end{array}$ & $\begin{array}{c}\text { Hybrid } \\
\text { method }\end{array}$ & OAD \\
Enhancement & - & $10^{7}-10^{9}$ & $10^{6}-10^{7}$ & $10^{6}-10^{8}$ & $10^{8}$ \\
Factors & & & & & \\
Substrate & $0.001 \times$ & $1 \times 1$ & $>2.5 \times 2.5$ & $>2.5 \times 5$ & $>2.5 \times 7.5$ \\
area (cm2) & 0.001 & & & & \\
Cost & Expensive & Inexpensive & Inexpensive & Inexpensive & Moderate
\end{tabular}

\subsubsection{Nanosphere lithography-based Substrates}

NSL can be used to fabricate wide area nanostructures on arbitrarily shaped substrates with relative ease. Nanosphere lithography, which was pioneered by Van Duyne et al., has been studied for the past few decades and is well established in the literature[78]-[80]. Achieving nanostructuring through NSL can be done using drop-coating, spin coating and trapping nanospheres at an air/liquid interface. Drop coating involves depositing a droplet of colloidal suspension of nanospheres onto a substrate. Spin coating adds a further step where this droplet is spun to achieve a desired thickness of coating. Trapping nanospheres at an air/liquid interface is a 2-step process where the self-assembly occurs at the air/liquid interface and they are then deposited preassembled onto a substrate[81]. After depositing nanospheres and forming the 2D array through one of the techniques described above, metal is deposited onto the substrate and the nanospheres are then removed.

The self-assembly process results in a monolayer. The resultant nanostructures formed from monolayers have a triangular geometry with the height equivalent to the deposition thickness of the material. Under special cases, dual layers of nanospheres are formed to create overlapped regions where the spacing in between the spheres results in a mask that includes circular openings to form round shaped nanostructures. 


\subsubsection{Nanoroughened/Nanoporous SERS substrates}

The first SERS signals were recorded from electrochemically roughened silver electrodes laying the foundations for nanoroughened SERS substrates. Since then, many studies have been performed on electrochemically roughened metal substrates. Nanowires/nanotubes can be trapped onto substrates to create nanoroughened SERS substrates [82], [83]. Polycarbonate membranes with varying pore-sizes can be used to create nanowires or nanotubes on a substrate by using electrochemical or electroless deposition of metal particles. Other types of membranes such as anodisic alumina membranes [84] are also widely used to create nanoporous of nanorougheded SERS substrates in a similar fashion. The membranes are usually discarded in such approaches. Nanoporous membranes have also been used as a base to support nano-films or nanoparticles to obtain sensitive SERS substrates. Au coated anodic aluminum oxide membranes (AAO) were developed with varying nano-pore sizes and different Au layer thicknesses [84]. The structure of AAO membranes is usually a tight hexagonal packing of cylindrical nanopores. Au nanoparticles aggregates were also impregnated into the cylindrical pores to perform label free molecular detection of nonresonant organic molecules[85].

Laser nanofabrication is another method used to create nanoroughened substrates. One method of using lasers is to induce aggregation or growth of noble metal particles through laser exposure [86]. Controlling laser power allows for adjusting nanoparticle size. Photolithography usually is limited by the wavelength of the laser light used (due to the diffraction limit of light) which does not allow creation of particles ( $<200 \mathrm{~nm}$ size). To overcome this limitation, pulsed laser sources are used to ablate the surface to create sub-diffraction limit features that can be used to create SERS substrates. Generally, a surface of the substrate is nanoroughened using a femtosecond laser and then a thin film of metal is deposited on the surface using vapor deposition techniques [87]. This results in the formation of nanoparticles with varying shapes and sizes.

Two phase materials have also been widely employed to create nanoporous SERS substrates. Mixed films of gold and silver (Au-Ag) have been chemically and electrochemically dealloyed to form nanoporous gold substrates. The size of the nanopores can be changed by controlling the dealloying times and temperatures [88]. It was found that smaller pore sizes created larger enhancements for such substrates. The sensitivity of the nanoporous Au-Ag films was increased 
by depositing them on polystyrene films, which were baked and annealed to create wrinkling [89]. Ordered arrays of nanoporous gold nanoparticles were also created by subsequent dewetting of metal films to create metal islands followed by dealloying to create porous metal islands on silica substrates. This allows for periodic multi-level nanostructures with large ordering of a few hundreds of nanometers (metal islands) and small ordering in the range of tens of nanometers (porous material of the metal islands) [90]. Oxygen plasma has been used to create silica nanoporous structures of free standing cylinders that were formed using mold imprinting techniques [91]. 
A

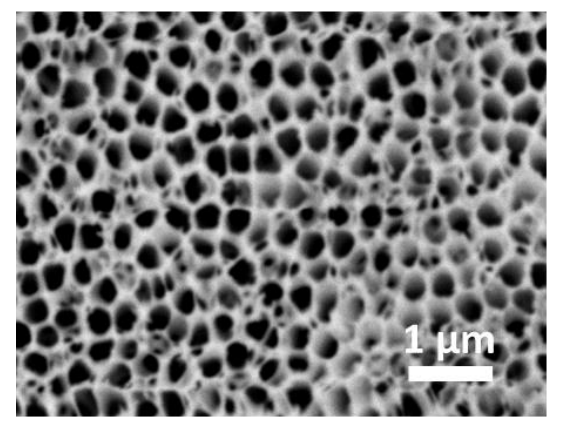

C

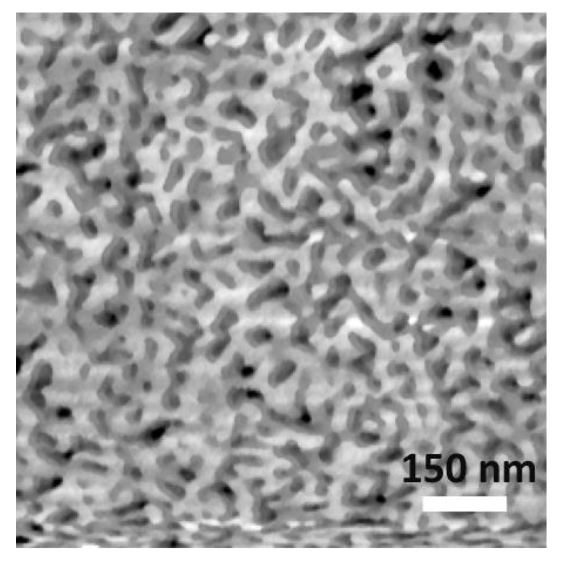

B

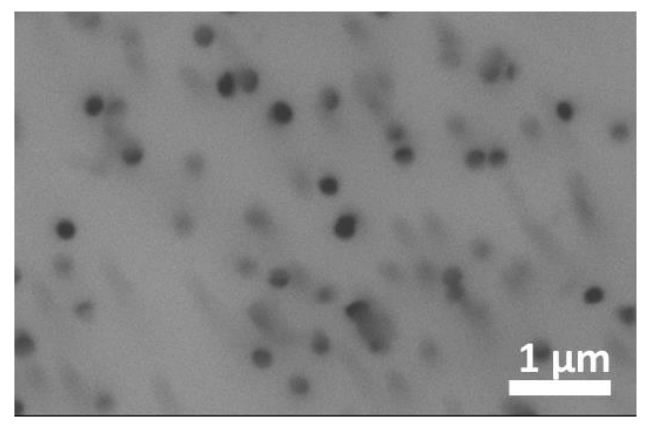

D
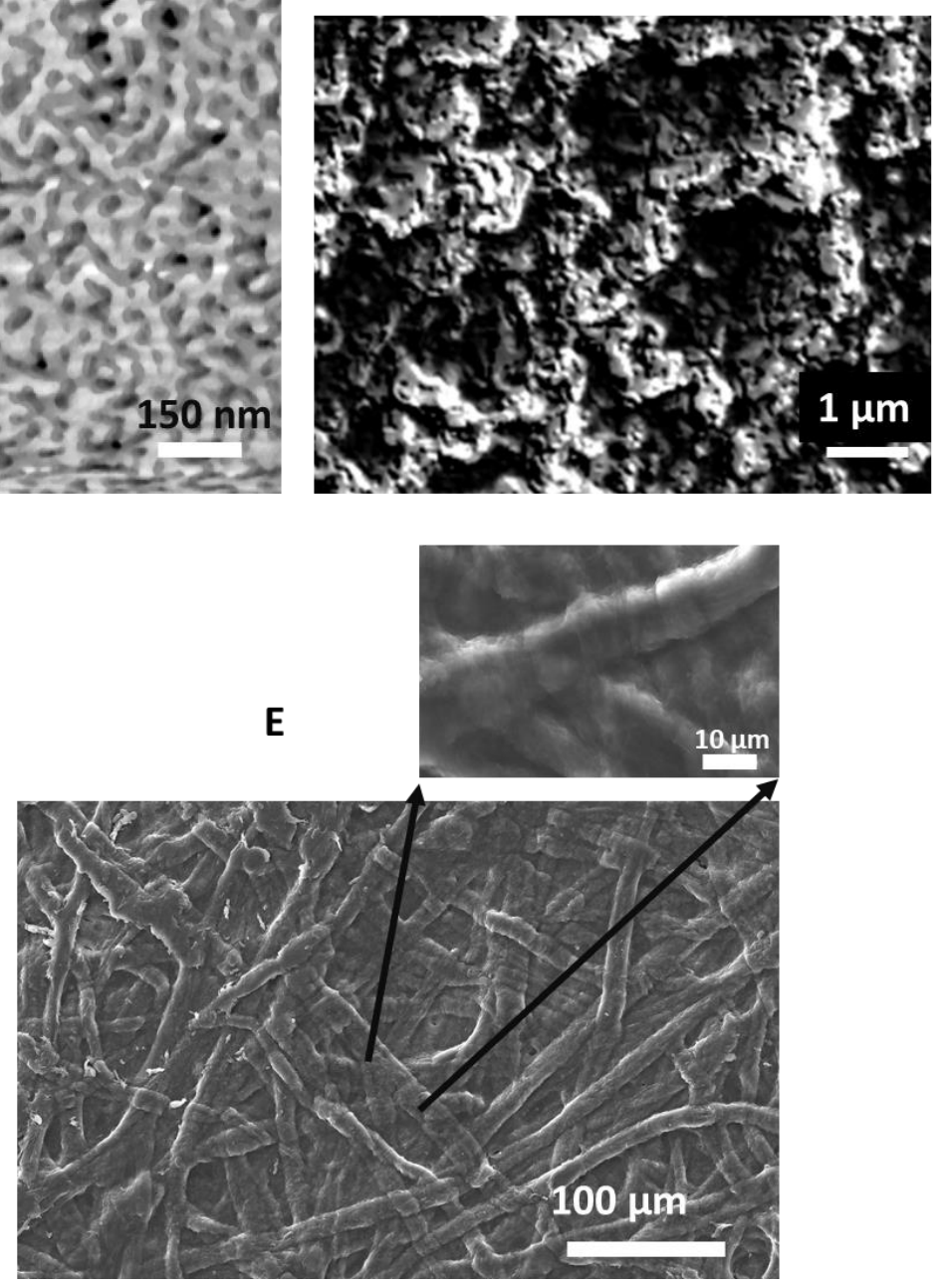

Figure 1-4 A) SEM image of nanoporous alumina substrate with $200 \mathrm{~nm}$ average pore size. B) SEM image of nanoporous polycarbonate substrate with $100 \mathrm{~nm}$ average pore size. C) AFM image of nanoporous Silica glass with $17 \mathrm{~nm}$ average pore size. D) SEM image Nanorough Silica glass fabricated using pulsed lasers(adapted from [92]). E) Cellulose membrane substrate 
Nanoporous silica (NPS) glass is another class of nanoporous substrates. They can be fabricated with tight control over the optical properties as well as the mechanical properties such as the porosity (linked to the pore size) and thickness [93]-[95]. They have been used to create substrates for recording holograms and also for point-of-care diagnosis of non-small cell lung cancer[94], [96]. These substrates provide a large surface area for interaction and are mechanically robust and chemically stable. Their usage as SERS substrates has not been widely explored. Example of few types of nanoporous structures are shown in Figure 1-4.

\subsubsection{Fiber based SERS}

A visual summary of various approaches to create fiber-optic SERS process is depicted in Figure 1-5. Early efforts on creating SERS fibers were based on coating optical fiber tips with dielectric microspheres which were coated with a noble metal[97]. Other widely used methods include sandblasting or roughening of a fiber core tip and coating it with a metal layer as well as creating metal islands over the fiber tip to induce SERS [98]. Slow deposition of silver was also employed to create nano-islands. This technique can be improved by creating large aspect ratio nanorods through oblique deposition of metal [99], [100] . Through chemical etching of a fiber-optic array, cavities were created which allowed sharp triangular structures (made of silica) to exist between neighboring fiber cores and metal was coated at an oblique angle of incidence for SERS [101]. Focused ion beam milling and electron beam lithography have also been used to create fine and precise periodic structures on fiber tips which can provide high tunability [102]-[104]. Laser induced self-assembly of nanoparticles at the surface of fiber has also been explored to create SERS optical fibers [105]. Pre-fabricated nanoparticles can be used in such an approach where the particles are trapped in a meniscus on the fiber tip and a laser is applied through the fiber to perform self-assembly at the tip of an optical fiber.

There have been a few efforts in using NSL to create plasmonic fiber optic platforms for general plasmonic and SERS sensing [106], [107]. NSL fabrication is a simple, inexpensive and tunable method for remote sensing SERS. Moreover, the ability of NSL to fabricate nanostructures on arbitrarily shaped geometries makes it suitable to create fiber-optic SERS substrates. To my knowledge, there have been a few efforts employing NSL to characterize optimal characteristics of fiber-optic sensors. 
A

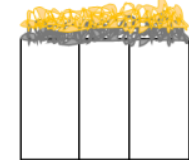

B
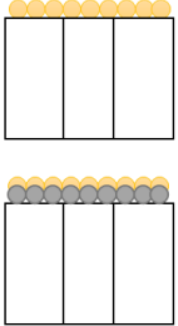

D
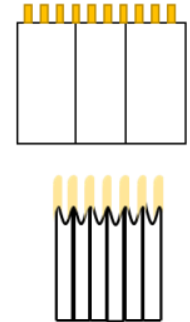

$\mathbf{F}$

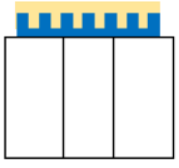

G

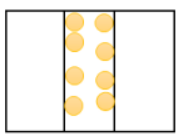

Mechanical, Electrochemical and Laser surface processing to form nano-roughened surfaces that are coated with noble metals.

Trapping nanoparticles on fiber tips, any shapes can be designed.

Self assembly of micro/nano spheres on fiber surface followed by metal coating (Metal film over nanosphere (MFON).

Electron beam assisted patterning to create sharp features with controlled geometry.

Imaging fiber bundle with selective etching of cores and metal deposition.

Polymer photolithography or imprinting followed by metal deposition.

Hollow core fibers impregnated with nanoparticles.

Figure 1-5 Various types of fiber-optic SERS sensing strategies.

\subsubsection{Differences in SERS spectra and Raman spectra}

Raman and SERS spectra acquired for a same chemical can exhibit differences. Generally, most of the molecules do not exhibit significant differences between their spontaneous Raman spectrum and SERS spectrum for the same excitation wavelength. Since the plasmonic enhancements vary for different wavelengths for different types of nanoparticles, this can result in wavelength dependent enhancement resulting in variation of peak heights. Also, the orientation of the adsorbed molecules with respect to the nanoparticle surface can affect the Raman vibrational mode resulting in spectral variations. The Raman cross sections can also be enhanced for the molecule due to the formation of metal-molecular complex systems. The polarization of the incident electric field also affects the SERS spectra more than standard Raman spectra since the plasmon enhancement depends on the polarization because of the shape of the nanoparticles used. Also, routine SERS 
measurements produce a broad background, called the background continuum which varies for each experimental condition. The background in spontaneous Raman is generated by autofluorescence of the molecule or impurities. There have been efforts to characterize the origin of background continuum in SERS but its origin in SERS is not clear. The studies have shown that the background is dependent on both the molecule and the metal used [108], [109].

\subsection{Theoretical Modeling of SERS}

Electromagnetic modeling of light propagation, scattering and attenuation within a medium elucidates modes of interaction of light with matter. For simple geometries such as spherical/cylindrical nanoparticles, analytical solutions exist but for complex irregularly shaped geometries, numerical computation is necessary. Some of the commonly used techniques for computational modeling are: Mie theory, transition matrix method, discrete dipole approximation (DDA), finite element method (FEM) and finite difference time-domain (FDTD) method [110].

Mie theory provides a solution to the Maxwell's equations for an incident plane wave scattered by isotropic, spherical and non-magnetic particles in an isotropic, non-absorbing medium. Since spherical objects are symmetrical, the scattered fields can be expressed by terms representing vector spherical harmonics [110]. Since these scattered fields are represented as a series sum, the accuracy of the final solution depends on the truncation of the terms. The $\mathrm{T}$ matrix method formulates a matrix that relates vector spherical harmonics of the incident and the scattered waves. The matrix elements depend on the refractive index of the particle as well as the media, the size and the orientation of the particles. For symmetrical elements, Mie theory or T Matrix method are usually faster as they do not require discretization of elements or specifications of inter-dipole spacing.

The DDA models each scatterer as an assembly of discrete dipoles reducing Maxwell's equations to an algebraic system of numerous coupled dipoles. The moment of each dipole is an assembly of responses from the incident electric fields and electric fields from neighboring dipoles. FEM and FDTD are the most flexible methods for simulating single nanoparticles and arbitrary shaped arrays of nanoparticles. FDTD employs rectangular grid of nodes and FEM uses non-rectangular mesh elements to compute the fields in each element. For simulating single particles, perfectly matched layers are employed to absorb the scattered radiation. For simulating periodic arrays of 
nanoparticles, periodic boundary conditions can be applied at the faces of elements in between the nodes to simulate a large array with reduced computational resources. FEM is superior to FDTD due to the use of irregular meshes that allow for complex geometries to be resolved with a relatively lower number of elements compared to FDTD.

FDTD simulations have been utilized extensively for performing SERS computations starting from single particles to periodic arrays of nanoparticles. It has been used for tuning SERS and also optimizing geometries to achieve large enhancement factors [111], [112]. FEM has also been employed for computing SERS enhancements for single particles and dimers [113]. For complex geometries such as curved nanotriangles formed by nanosphere lithography, FDTD has been used on various occasions [114]-[116] to compute plasmonic enhancements due to nanotriangles created using nanosphere lithography. There have been limited efforts in using FEM to simulate electromagnetic enhancements in periodic array of curved nanotriangles formed using nanosphere lithography.

\subsection{Motivation}

Raman spectroscopy has been used as a chemical fingerprinting technique for almost a century by the virtue of probing molecular vibrations. RS requires minimal to no sample preparation, it can probe aqueous media (water interferes with near-infrared/infrared absorption spectroscopy at various ranges), it requires monochromatic excitation source, it can collect spectra using optical fiber probes and data collection is usually performed in a backscattered or reflection configuration. In comparison to other spectroscopy techniques, the spectral information obtained from RS contains distinct peaks and features that are suitable for chemometric analysis as shown in Figure 1-6. RS is also sensitive to the physical and chemical changes occurring in cells in response to various diseased conditions[117]. It can probe physical changes such as change in the nucleus-tocytoplasm ratio, changes in chromatin fiber and overall density changes [118]. It can also probe chemical changes associated with higher metabolic activity, changes in lipids, proteins and various biomolecules [117], [119]. These characteristics are highly sought after for rapid and portable point-of-care diagnostic devices. 

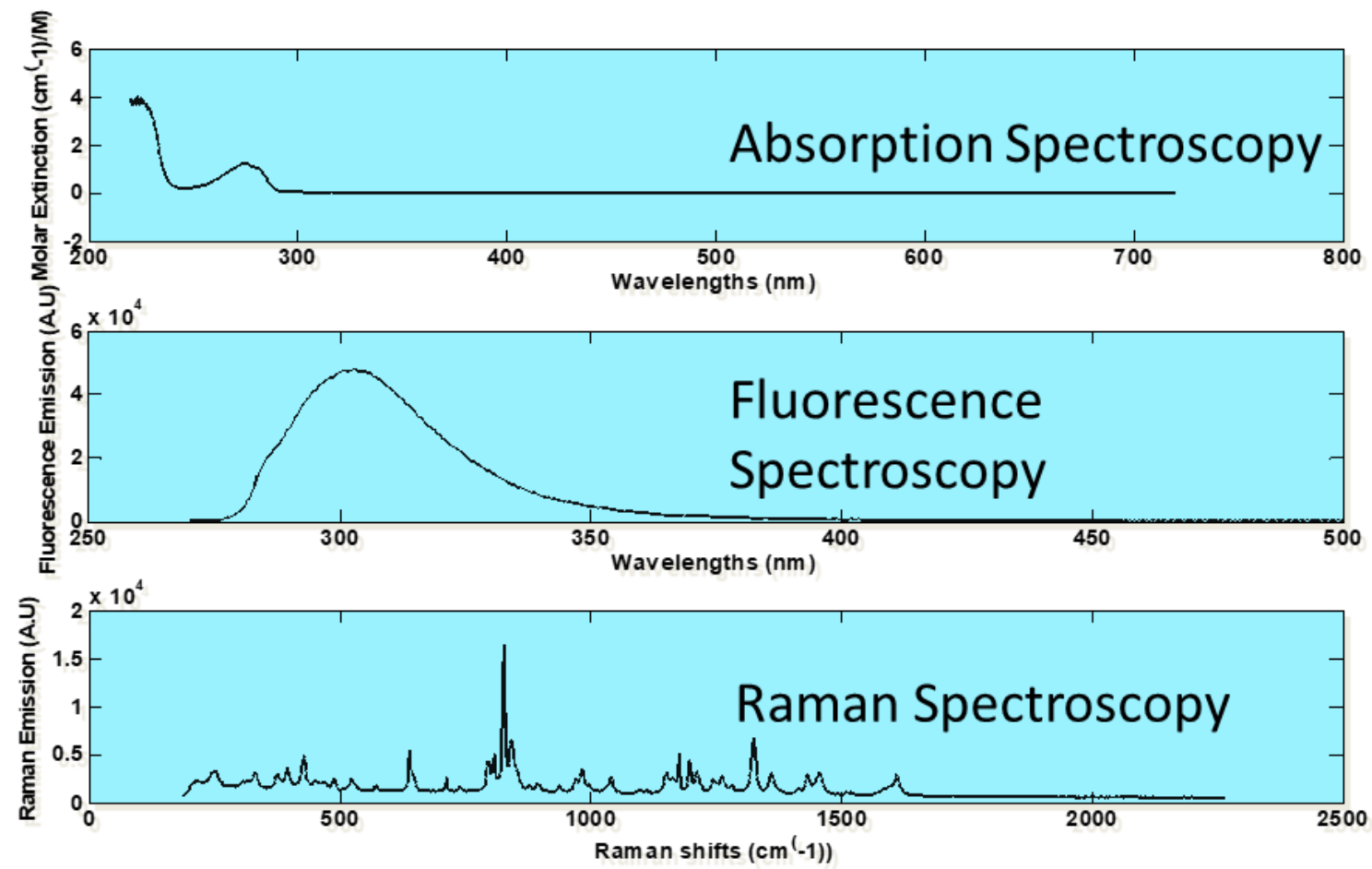

Figure 1-6 Comparison of spectra obtained using absorption, fluorescence and Raman spectroscopies. RS contains distinct peaks and features that are suitable for chemometric analysis

RS has also been widely employed in non-medical applications such as explosives detection, industrial process analysis, crystalline structure analysis and to detect changes associated with diseased conditions in-situ with high clinical specificities and sensitives using non-invasive or minimally invasive endoscopic approaches. However, there have been limited attempts to translate RS from research stage to fully functional clinical diagnostic devices due to the low probability of Raman scattering in biological media as biomolecules are weak Raman scatterers, and due to the interference from tissue generated auto-fluorescence. This results in long acquisition times for RS compared to fluorescence spectroscopy and reflectance spectroscopy which makes it quite challenging to use in motion prone environments. In-situ diagnostics are valuable for detection of diseased margins or surgical resection margins. Endoscopy is employed to perform in-situ diagnostics and fluorescence endoscopy is widely used in clinics. RS based endoscopy is challenging as miniaturization of the probes can result in lower signal quality due to the interference of fluorescence generated by the optical fibers used. Several probes have been 
designed for endoscopic use and they generally employ optical filters to reduce the background generated by silica fibers [53]. Due to the use of these filters, it is harder to miniaturize the probes which makes interstitial applications challenging. Hence, endoscopic usage can be challenging and RS imaging in such environments has been challenging.

SERS amplifies standard Raman spectra resulting in highly sensitive detection of chemicals with reports of detection at the single molecule level (for ideal cases such as resonant molecules with large Raman cross sections). Clinically, SERS can be used to monitor disease related variations in biofluids or perform in-situ tissue analysis. Biofluid analysis is relatively easier and any mode of SERS can be employed to perform analysis. The primary mode of clinical application for SERS has been through injecting nanoparticles with Raman active dyes that have large Raman cross sections (nanotags) which can be actively targeted (special surface chemistry) or passively targeted (through localized or intravenous injection). It has been shown that multiplexing can be achieved with relative ease when using such nanotags[70]. Fiber based endoscopic devices are utilized for in-situ measurements of SERS spectra[70], [120]. Intravenously injected passively targeted nanoparticles have been found to accumulate in liver and actively targeted nanoparticles have been found to accumulate in targeted regions for large periods of time, even after the concentration of these particles in blood has returned to normal[121]. Since these SERS applications requires nanoparticles, long term cytotoxic analysis needs to be performed to evaluate the safety of using nanotags for SERS. The nanoparticle suspensions, while convenient, is plagued by uncontrolled aggregation of nanoparticles, and Brownian motion of nanoparticles which creates variability in acquired SERS signals.

Planar SERS substrates are ideal for performing ex-vivo analysis of biofluids or tissues. These platforms have nanoparticles trapped or embedded on their exposed surfaces. In contrast to nanoparticles in suspensions, the substrates allow for better control over inter-particle spacing and allow for the creation of periodic arrays that increase enhancements. The nano patterns created on the substrates are controlled to have regular periodicity (fixed surface area and number of particles) thus increasing the possibility of quantitative analysis. Since the samples can be directly placed on the substrates or the substrates attached to a sample surface, they can be beneficial for passively targeted SERS detection (as analyte can be introduced directly on nanoparticle sites) and for localized application of SERS. Periodic substrates are usually harder to fabricate, and they involve 
either masks for patterning or electron beam lithography. Since the fabrication geometry is controlled, reproducible substrates can be created, and quantification is generally possible. Nonperiodic substrates are usually fabricated using mechanical abrasion, electrochemical etching or high-power pulsed laser abrasion of the surface followed by metal evaporation or nanoparticle coating. Such methods result in low average enhancements compared to periodic substrates, but in contrast, they have larger surface areas where SERS can be performed, and they can be inexpensive to manufacture.

Fiber based SERS substrates present a unique opportunity to temporarily introduce nanoparticles to the region of interest. The tip of the fiber can be fabricated into a nano-platform which allows SERS to be employed for biofluid analysis or tissue analysis. Such fibers can be paired with the current generation of endoscopic devices to create a multimodal diagnostic technique. These fibers can also be used to perform biofluid analysis and interstitial sensing in-situ. Various types of nanopatterns have been employed on the surface of optical fibers as described before, but few approaches have employed NSL for creating optical fibers with a periodic array of nanotriangles for remote SERS sensing.

NSL is an inexpensive technique but it usually results in sparsely spaced nanoparticles. In comparison, nanoporous substrates can allow for a large surface area for SERS. Silica nanoporous glass is mechanically robust and chemically inert substrate that can be utilized for SERS. The porous structure is created using a combination of chemical etching and annealing which is well controlled. Standard optical fibers can also be made from borosilicate glass that can be changed into nanoporous glass. This can create a highly sensitive and mechanically robust fiber SERS substrate for remote sensing.

For this thesis, air-water based fabrication using NSL was performed and characterized. A custom fabrication setup was created to fabricate SERS optical fibers with nanoparticle arrays on the fiber tips. A specialized RS collection setup was utilized for collection of spectra from the entire surface of an optical fiber. A remote sensing fiber-optic based SERS platform was created and various fibers with different numerical apertures were tested to find an optimal SERS substrate. Nanoporous Silica (NS) glass was utilized to create a nanoporous SERS substrate by depositing gold layers on the surface. An optimal Au coating thickness was found, and concentration 
dependence of SERS was evaluated. This nanoporous structure can be designed on optical fiber tips to create remote fiber optic SERS sensors. Theoretical simulations are performed to evaluate the performance of NSL and nanoporous SERS substrates.

\subsection{Hypothesis and objectives}

Hypothesis: Disposable fiber-optic SERS probes used for remote sensing can improve on RS fiber optic probes.

The following were specific objectives:

1) Fabricate and optimize fiber-optic SERS platforms using NSL.

a. Fabricate flat NSL substrates for characterization of nanoparticles.

b. Fabricate optical fiber NSL substrates based on protocols identified by flat NSL substrates.

c. Test various numerical apertures (NAs) of optical fibers for SERS enhancements.

d. Compute SERS enhancement factors.

2) Fabricate and optimize nanoporous SERS platforms.

a. Characterize nanoporous surface using AFM and evaluate pore size distributions.

b. Perform sputter coating of substrates with different thickness of Au to make SERS active substrates.

c. Evaluate an optimal Au coating thickness as compute enhancement factors.

3) Theoretically model NSL and nanoporous SERS platforms.

a. Fabricate single curved nanotriangle using CAD software to mimic fabricated geometries.

b. Compute extinction spectra, maximum and integrated enhancement factors for different heights and sizes of nanoparticles.

c. Fabricate nanoporous structure using CAD software to mimic nanoporous surface.

d. Compute maximum and integrated enhancement factors for nanoporous films.

4) Test Raman spectroscopy clinically

a. Acquire spectra from excised breast tissues and evaluate differences in normal and diseased regions.

b. Evaluate differences in spectra from freshly excised tissues and processed tissues. 


\section{Methods}

\subsection{Instrumentation for Raman spectroscopy}

A custom designed Raman collection setup optimized for excitation and collection was used, Figure 2-1 A\&B provides a schematic of the setup. A 40X microscope objective (0.65 NA, Motic, China) was used to focus a collimated laser (785 nm, Ondax Inc, USA) into a $30 \mu \mathrm{m}$ diameter spot size (measured using a CMOS camera chip placed at the focus). The excitation spot was centered on the sample area and a dichroic beam splitter was used to direct light to and from the sample into a spectrometer. A fiber coupled high throughput spectrometer (16 bit digitization, Hyperflux PRO, Tornado Spectral Systems, Canada) was used to collect the Raman spectra. Calibration of the spectrum was performed using a silicon disc to account for laser variations. Laser power was measured at the sample using a thermal power sensor (PM160T, Thorlabs, USA). The sample was placed on a 3D translation stage (MTS50-Z8, Thorlabs, USA) with travel increments ranging from a few tens to hundreds of microns. The list of components used in the Raman setup are provided in Figure 2-1 C.

For SERS optical fiber substrates, the configuration was slightly modified to accommodate for the large area of the optical fibers as shown in Figure 2-1 B. Since optical fibers have two faces and one of the faces was used to create SERS nanopatterns, direct and remote collection was performed by switching the faces through which they are excited as shown in Figure 2-1 B. The optical fibers were positioned using the translation stage to optimize the collection. 

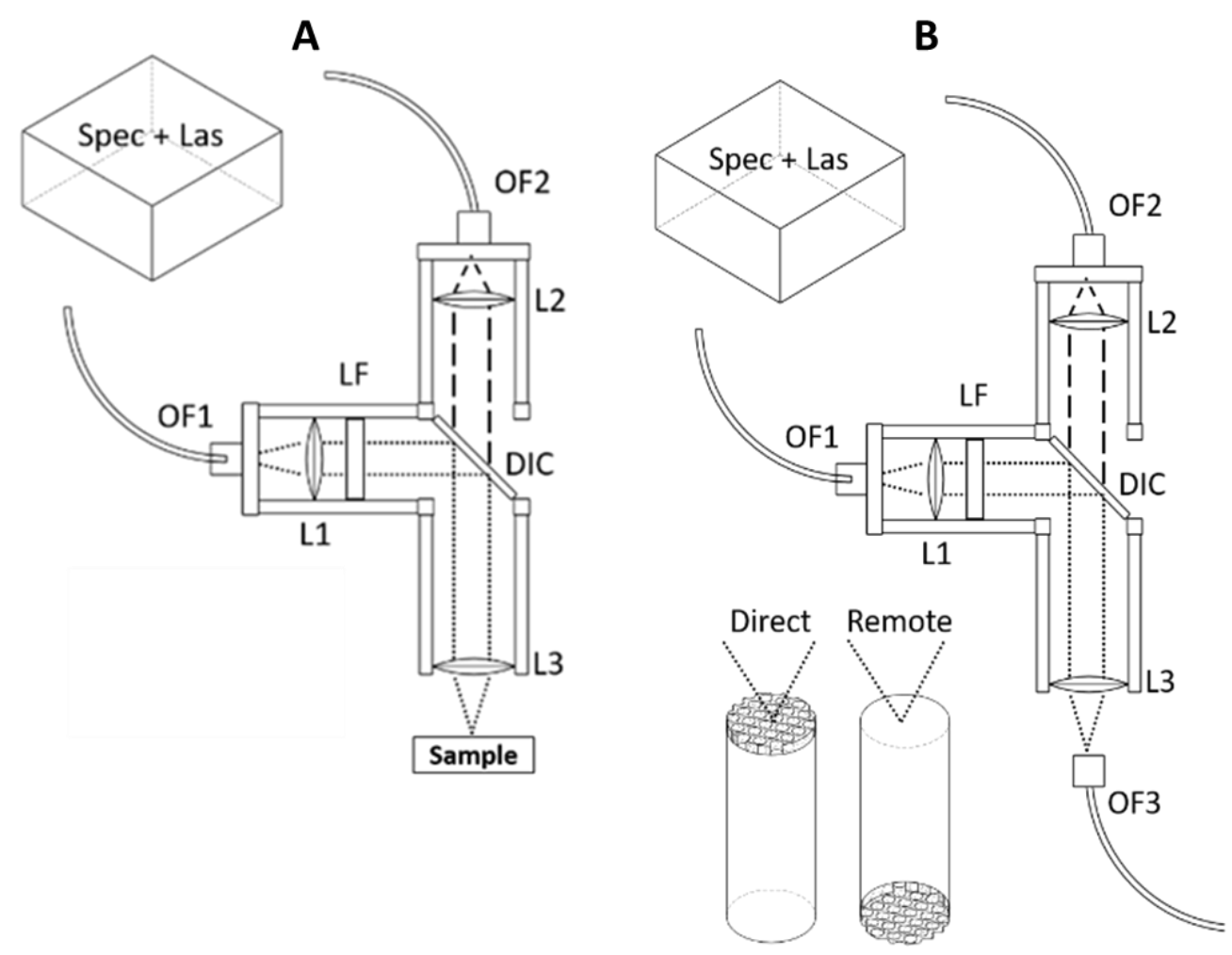

\section{C}

\begin{tabular}{|l|l|l|}
\hline Items & Flat samples & Fiber probes \\
\hline OF1 & \multicolumn{2}{|c|}{$105 \mu \mathrm{m}(0.22 \mathrm{NA})$} \\
\hline OF2 & $300 \mu \mathrm{m}(0.22 \mathrm{NA})$ & $1 \mathrm{~mm}(0.22 \mathrm{NA})$ \\
\hline OF3 & \multicolumn{2}{|c|}{$12.5 \mathrm{~mm}(0.25 \mathrm{NA})$} \\
\hline L1 & \multicolumn{2}{|c|}{$25 \mathrm{~mm}(0.22 \mathrm{NA})$} \\
\hline L2 & \multicolumn{2}{|c|}{ Motic 40x (0.65NA) } \\
\hline L3 & \multicolumn{2}{|c|}{$12.5 \mathrm{~mm}$ laser line filter } \\
\hline LF & \multicolumn{2}{|c|}{$25 \mathrm{~mm}$ dichroic edge filter } \\
\hline DIC & Hyperflux PRO, Tornado spectral systems \\
\hline Spec & \multicolumn{2}{|c|}{ 785nm 500mW } \\
\hline Laser & \multicolumn{2}{|c|}{. } \\
\hline
\end{tabular}

Figure 2-1 Using a $105 \mu \mathrm{m}$ optical fiber (OF1), laser light is collimated using lens (L1) and filtered using a band pass laser line filter (LF). The collimated laser light passes through a dichroic mirror (DIC) which reflects the laser lights on sample. Red shifted wavelengths passed through the DIC and another lens (L2) focused light into a $1 \mathrm{~mm}$ optical fiber which is connected to a Raman spectrometer. A) The laser was focused on the sample (flat substrates, biological tissues) to collect RS and SERS spectra B) The SERS optical fiber (OF3) is excited in the direct and remote configurations to collect SERS spectra. C) Description of optical elements used in the setups. 


\subsection{Data processing}

\subsubsection{Optical fibers}

All the SERS intensity comparisons were performed on raw spectra and background suppression was mainly used for visualizing the peaks. An iterative algorithm with non-negative constraint (to keep intensity values positive) was used to match the background spectra to the collected SERS spectra. The background spectrum was iteratively scaled by a factor. The factor was initialized to 1 and in each iteration, the factor was increased or decreased by 0.001 depending on the background being larger or smaller than the SERS spectra until the difference between the background and the SERS spectra was minimized and without any negatives (Figure 2-2). This allowed to perform an optimized matching of the fiber backgrounds to the SERS spectra.

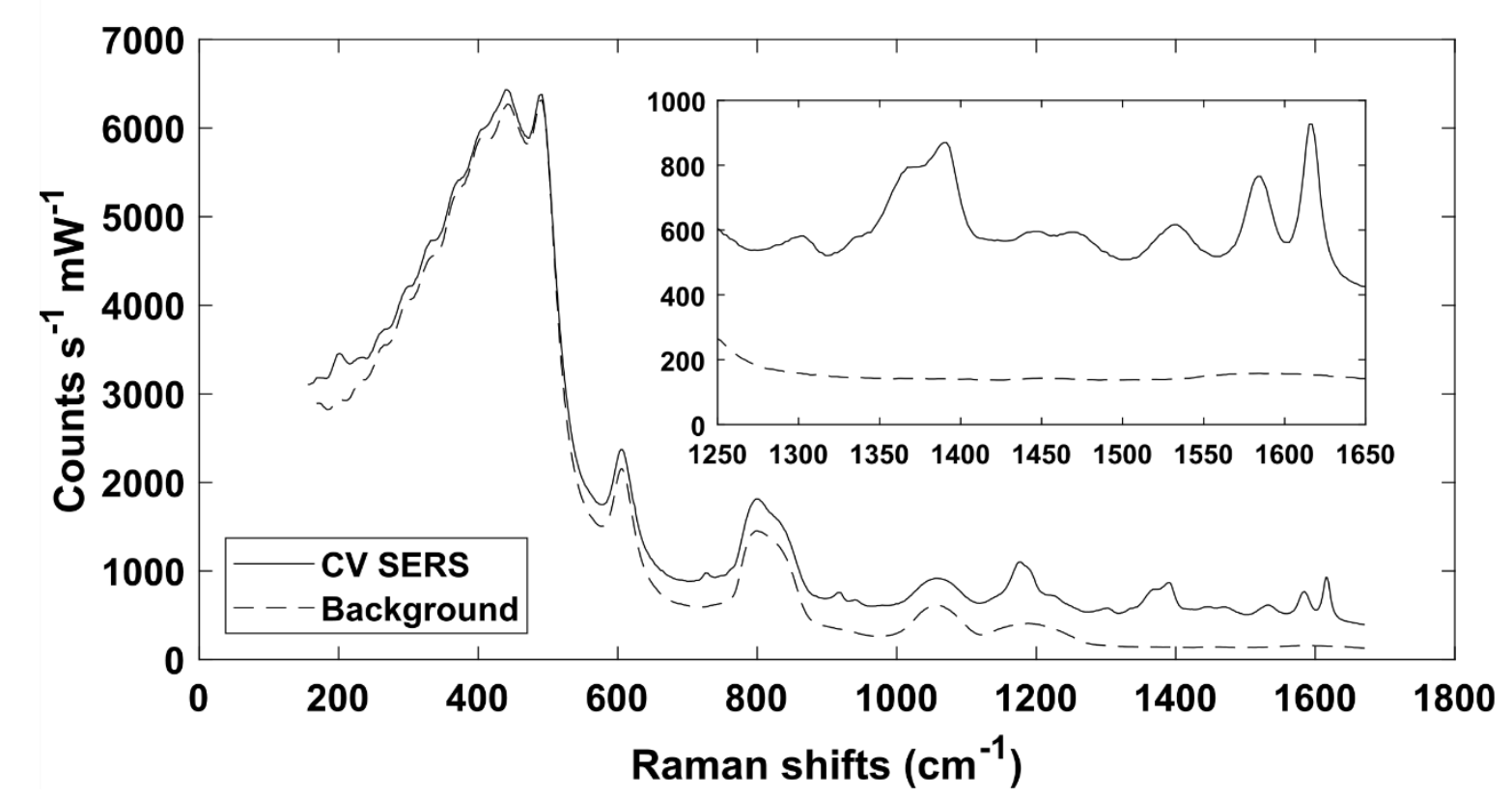

Figure 2-2 Background suppression method used for optical fibers. The inset shows an expanded view of the Raman shift range $1250 \mathrm{~cm}^{-1}$ to $1650 \mathrm{~cm}^{-1}$ to display the background and SERS spectra.

\subsubsection{General background estimation}

For estimating complex backgrounds, a sliding window $2^{\text {nd }}$ order polynomial based on a locally weighted regression model (for suppressing outliers, which represent Raman peaks) was first used to estimate the background of the spectra. A window size of 130-200 spectral points was chosen 
to find an optimized background and the background suppression method described above was applied using the smoothed background spectra. The function was implemented in MATLAB TM (Mathworks, USA) using the smooth library function with the option "loess". An example of the background suppression process is shown in Figure 2-3.

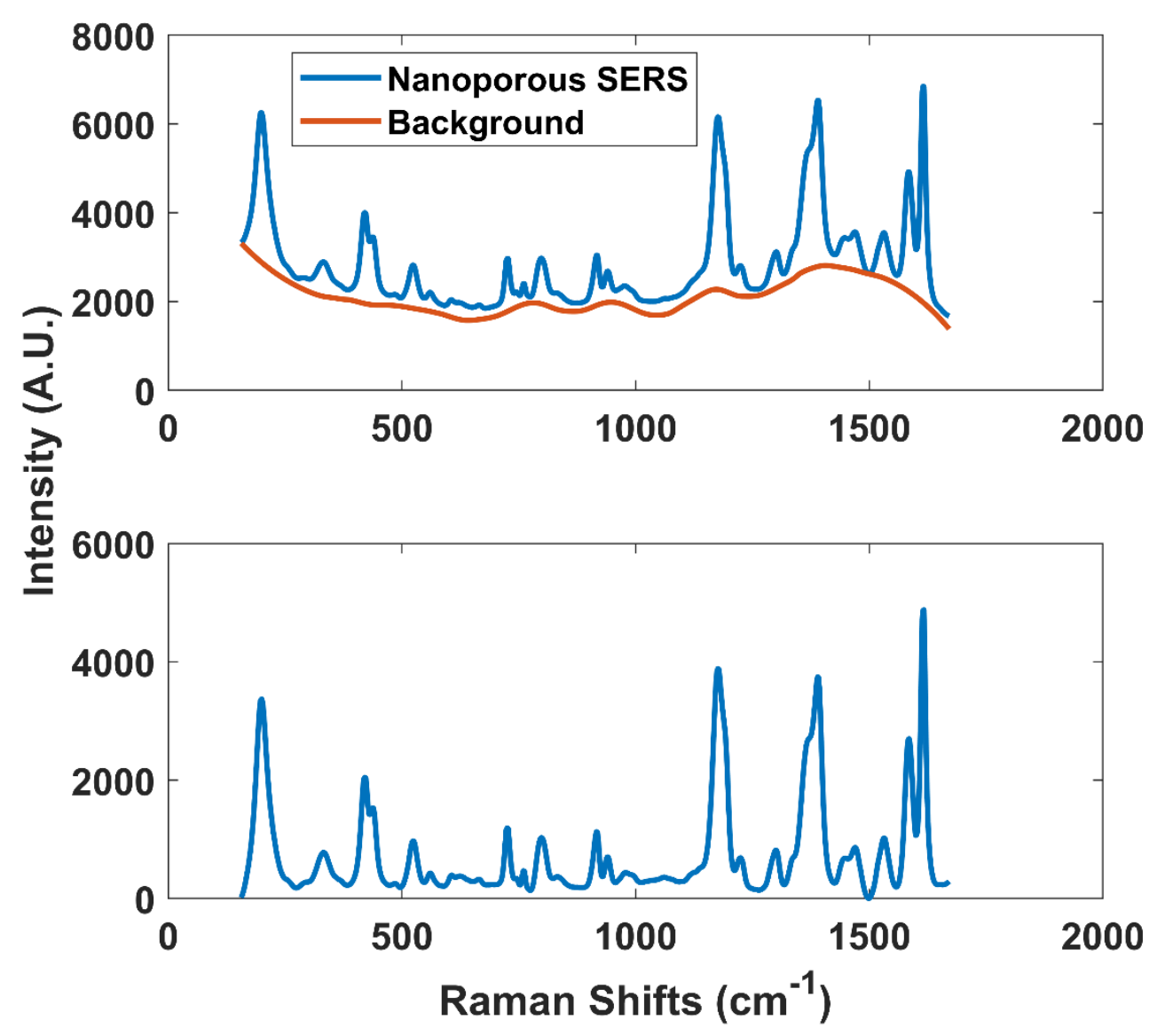

Figure 2-3 Background suppression method used for general background suppression. The top plot represents the raw SERS spectra in blue and estimated background in red. The bottom plot represents background corrected spectra.

\subsubsection{Nanoporous size estimation}

The nanoporous structure of NS glasses did not have any specific orientation of the pores. To analyze the pore size, radial profiles were extracted from the AFM image in a spoke-wheel pattern as shown in Figure 2-4. A $2^{\text {nd }}$ degree polynomial was fit to the profiles to perform non-linear detrending caused by the curvature of the surface on a nanoscale. Zero crossings were used from 
these profiles to obtain the distribution of the pore sizes. Pore sizes smaller than $3 \mathrm{~nm}$ were discarded as noise.

A

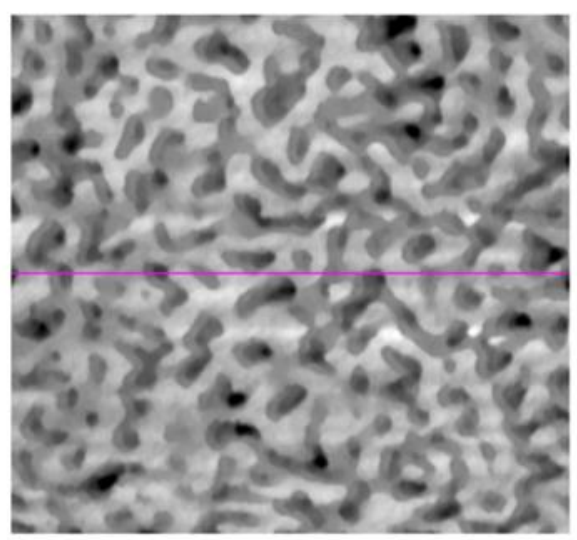

B

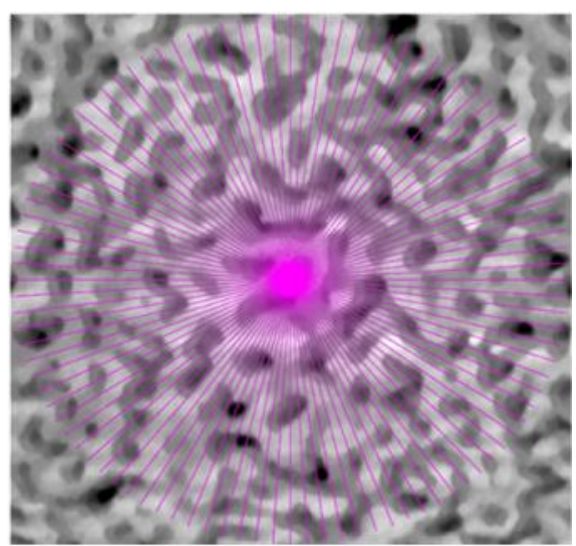

C

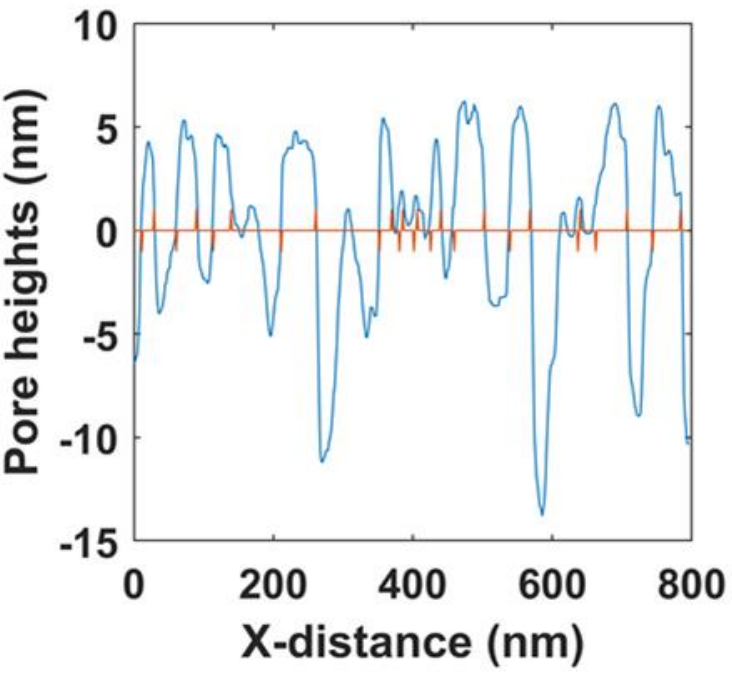

Figure 2-4 A) Central line profile overlaid on the image B) Radial line profiles extracted for estimating pore size C) Line profile corresponding to the central line in A.

\subsubsection{Area under the curve for spectral peaks}

For comparison of data, area under the curve (AUC) was computed for the peak of interest. The end points of the peaks were chosen to find a linear baseline (tangent) and AUC was computed for the shaded region as shown in Figure 2-5. 


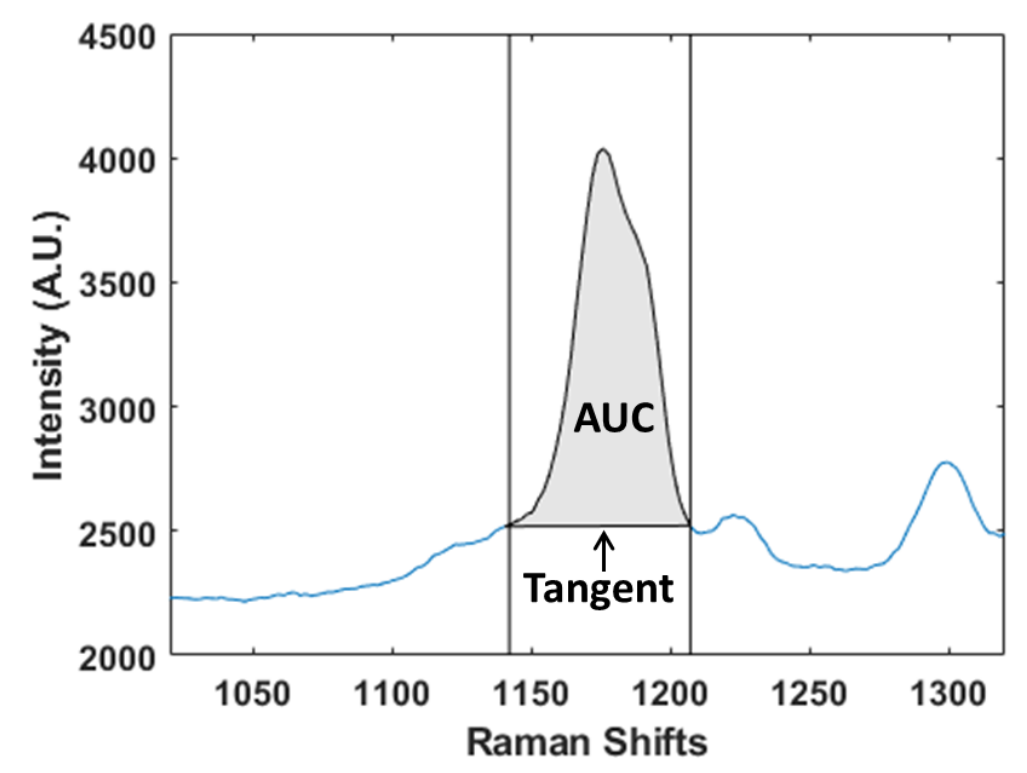

Figure 2-5 Schematic for computing area under the curve using a linear tangent connecting the end points of the peak.

\subsection{Fabrication - Nanosphere lithography}

\subsubsection{Surface preparation}

\section{Fiber-optic substrates}

Optical fibers are made of silica glass as a concentric core, cladding and coating/buffer (for support). For $200 \mu \mathrm{m}$ core optical fibers used in this study, the cladding is $25 \mu \mathrm{m}$ thick and the coating ranges from $100 \mu \mathrm{m}$ to $275 \mu \mathrm{m}$ as shown in Table 2-1. The light is guided through the core of the optical fibers and this core is responsible for generating fluorescence and Raman photons that interfere with the measurements obtained through the distal end of the fiber. The optical fibers used in this study contain a low $\mathrm{OH}$ concentration which was found to provide the lowest fluorescence/Raman background [122].

Table 2-1 Optical fiber parameters

$\begin{array}{ccccc}\text { Fiber type } & \text { NA } & \text { Core } & \text { Cladding } & \text { Coating } \\ \text { FG200LEA } & 0.22 & 200 \mu \mathrm{m} & 220 \mu \mathrm{m} \text { (Fluorine-doped silica) } & 320 \mu \mathrm{m} \text { (Acrylate) } \\ \text { FT200EMT } & 0.39 & 200 \mu \mathrm{m} & 225 \mu \mathrm{m} \text { (Hard TECS) } & 500 \mu \mathrm{m} \text { (Tefzel) } \\ \text { FP200ERT } & 0.5 & 200 \mu \mathrm{m} & 225 \mu \mathrm{m} \text { (Hard Polymer) } & 500 \mu \mathrm{m} \text { (Tefzel) }\end{array}$


For optical fibers, the tips were cleaved to achieve a clean flat surface before surface preparation. The polymer coating of the optical fibers was stripped off using a fiber stripper of an appropriate size to match the core and cladding diameter of the fibers. The stripped fiber was guided through a bare fiber adapter so that $2 \mathrm{~cm}$ of the stripped fiber protruded outside the adapter. This part of the fiber contains the core and the cladding which was made of silica glass. A sharp ruby optical scribe (S90R, Thorlabs, NJ, USA) was placed with its flat surface resting onto the bare fiber adapter's face. A small score was created on the fiber surface with the optical scribe and the fiber was pulled to break at the score line as shown in Figure 2-6.

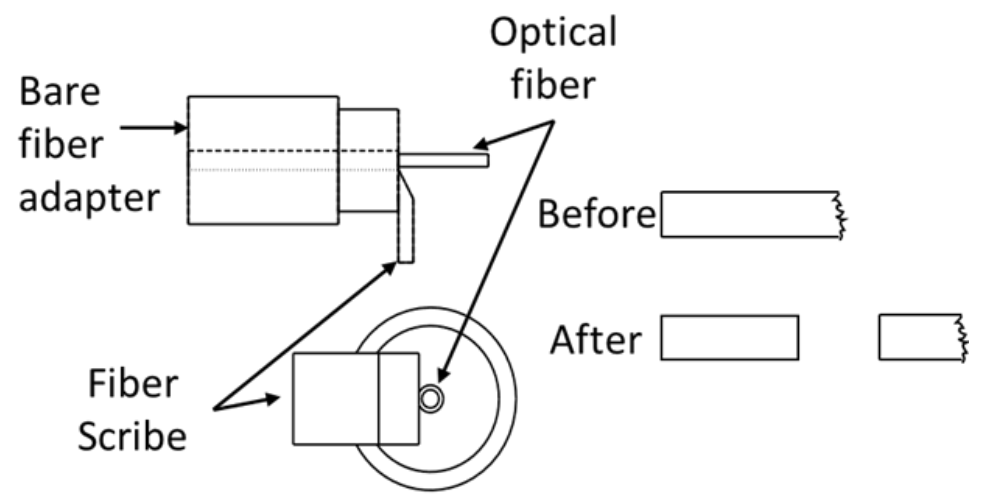

Figure 2-6 The Process of creating a flat cleaved fiber tip: 1) An optical fiber is cut using scissors and the buffer is removed. The surface of the optical fiber is jagged (depicted by the before image). 2) The fiber is inserted into the bare fiber adapter with $1 \mathrm{~cm}$ of fiber tip with no buffer protruding from the adapter. 3) A fiber cleaver is used with the flat bed of the blade resting on the face of the bare fiber adapter to create a score on the fiber perpendicular to its central axis. 4) The fiber is mechanically pulled to create a flat cleave on the fiber tip (depicted by the after image).

\section{Flat glass substrates}

The flat glass substrates were used as obtained from a manufacturer (VWR, USA - $22 \mathrm{~mm} \times 22$ $\mathrm{mm} \times 0.17 \mathrm{~mm}$ ). Surface preparation of the substrates (both optical fibers and flat substrates) were done by sonicating them in ethanol solution for 5 minutes, rinsing with Milli-Q ultrapure water (Millipore Sigma, USA) followed by oxygen plasma treatment (5 minutes - High setting, PDC-32 G - $150 \mathrm{~W}$, Harrick Plasma, USA) to further prepare the substrates for NSL by increasing their hydrophilicity. 


\subsubsection{Nanosphere lithography on flat glass substrates}

Glass microscopic cover slips (VWR, USA) of size $22 \mathrm{~mm} \times 22 \mathrm{~mm} \times 0.17 \mathrm{~mm}$ were used as flat substrates. Polystyrene (PS) spheres were purchased from Life technologies (CML latex, $1 \mu m$ ) with a $4 \% \mathrm{w} / \mathrm{v}$ concentration. The solution containing PS spheres was diluted with an equal volume of ethanol to create a dispersion solution. The substrates were prepared using drop coating with assembly at air-water interface. For the drop coating, a $5 \mu \mathrm{L}$ drop of PS was applied to a prepared glass slide as shown in Figure 2-7 (A). The substrates were dried under normal ambient conditions.

To assemble PS microspheres at an air-water interface, a specialized polytetrafluoroethylene (PTFE) tank was fabricated having a plunger mechanism as shown in Figure 2-7 B. The PTFE tank was filled with ultrapure water and a glass cover slip was introduced into the water filled tank. The volume of a dispersion solution was calculated that contained PS particles at a slightly higher number than the number of particles required to form a monolayer covering the surface of the tank. The diluted PS microsphere solution was slowly applied to the surface of water in the tank using a micro syringe with a 31-gauge needle. The PS spheres gradually spread on the surface of water and an iridescent (due to the interference of the tightly packed monolayer of PS spheres) layer was visually observed. A plunger was used to compress the PS monolayers to remove any gaps in the monolayer. The glass slide was slowly pulled out of the tank at a rate of $10 \mu \mathrm{m} / \mathrm{s}$ using a translation stage (MTS50-Z8, Thorlabs, NJ, USA) until the slide was fully out of the water as shown in Figure 2-7 (B). 
A

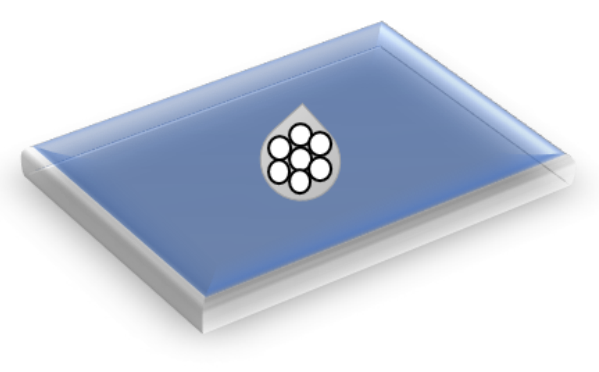

B

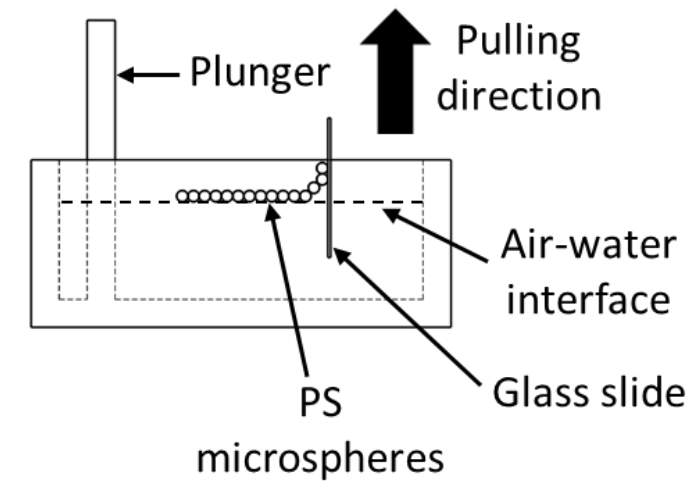

Figure 2-7 A) Drop coating method of NSL fabrication, and B) Air-water interface based NSL fabrication method for flat substrates. The PS spheres formed a monolayer at the air-water interface and the PS spheres anchored onto the glass slide. The slide was slowly pulled out of water to transfer the monolayer onto the surface of the glass.

\subsubsection{Nanosphere lithography on optical fiber substrates}

Standard silica based optical fibers (Table 2-1) were purchased from Thorlabs (NJ, USA) and were cut to $15 \mathrm{~cm}$ length. A fiber stripper tool was used to strip the coating from both ends. To perform NSL, plasma treated optical fibers were placed in a PTFE tank filled with water and an optical fiber was lowered into the water at an oblique angle using a 3D translation stage (MTS50-Z8, Thorlabs, NJ, USA) as depicted in Figure 2-8. PS monolayer was formed on the surface like the method described in the previous section. Once a monolayer was achieved, the optical fiber was pulled out at a steady rate of $10 \mu \mathrm{m} / \mathrm{s}$ as depicted in Figure 2-8. The slow draw of the optical fiber allows the spheres to be coated onto the fiber tip gradually transferring the monolayer from the air-water interface onto the fiber tips. The optical fiber tips were imaged after the NSL process and the images were thresholded to estimate coverage area. The optical fiber with the best coverage was chosen as a reference fiber and area ratios were computed for each optical fiber. These ratios were used to normalize the SERS intensity 


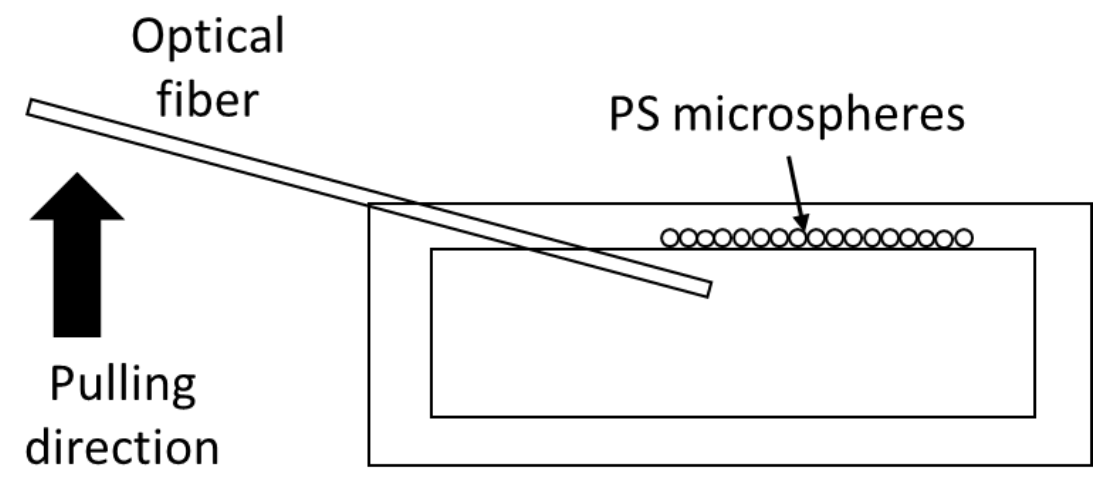

Figure 2-8 The air-water interface based NSL fabrication method for optical fiber tips. PS monolayer was formed at the air-water interface. The optical fibers were pulled in the upward direction and the monolayer was transferred onto the tip of optical fibers.

\subsubsection{Removal of nanospheres}

The sputter coated substrates were immersed in a 50\% ethanol solution contained in an ultrasonic (1800, Branson, USA) water bath. The flat and fiber substrates were held inside the beaker through tethered strings such that they do not touch each other or the walls of the container. The substrates were sonicated at the max pressure setting for 2 minutes for the removal of nanospheres.

\subsection{Fabrication - Nanoporous substrates}

Nanoporous silica (NPS) glass substrates were acquired from the Department of Photonics and Optical Information Technology, ITMO, St. Petersburg, Russia. NPS glass substrates were made from glass which had a composition of $\mathrm{Na}_{2} \mathrm{O}-6.2 \% ; \mathrm{B}_{2} \mathrm{O}_{3}-25.1 \%$; $\mathrm{SiO}_{2}-67.8 \%$ [93]. The glass had a chemically unstable borate phase intertwined with a chemically stable silica phase. Due to the unstable nature of the borate phase, it can be removed using an acid solution. After removal of the acid solution, the glass was annealed, mechanically shaped and processed with an alkali treatment to form NS glasses with varying properties such as pore size and overall porosity. The characteristics of NS glasses are depicted in Table 2-2. 
Table 2-2 Characteristics of Nanoporous glasses

\begin{tabular}{|c|c|c|}
\hline Characteristic & NS-7 & NS-17 \\
\hline Average pore size (nm) & 7 & 17 \\
\hline Porosity (\%) & 26 & $52-54$ \\
\hline Specific surface area $\left(\mathrm{m}^{2} / \mathrm{g}\right)$ & \multicolumn{2}{|c|}{$100-120$} \\
\hline
\end{tabular}

\subsection{Sputter coating of substrates}

Denton Desk IV (Denton Vacuum, USA) was used to coat the prepared NSL substrates with different thicknesses of gold. Flat substrates were placed onto the central part of the sputter stage and the fibers were attached to the edge of the stage to hold them vertically such that the tips were parallel to the sputter stage as shown in Figure 2-9. The thickness was controlled by varying the time of sputter (30s-3min). A pressure of 100 mTorr was employed and $50 \%$ setting for the current was used on the device to perform sputtering. Argon gas was used as the sputtering environment. To improve the adhesion of gold onto silica substrates, a base layer of either titanium, chromium, or oxide layers are used [123].. Using titanium or chromium can have adverse effects on the SERS enhancements and oxide layers introduce a new step in the manufacturing process. Sputtering was found to be suitable to create stable nanoparticles on silica substrates for this study. 


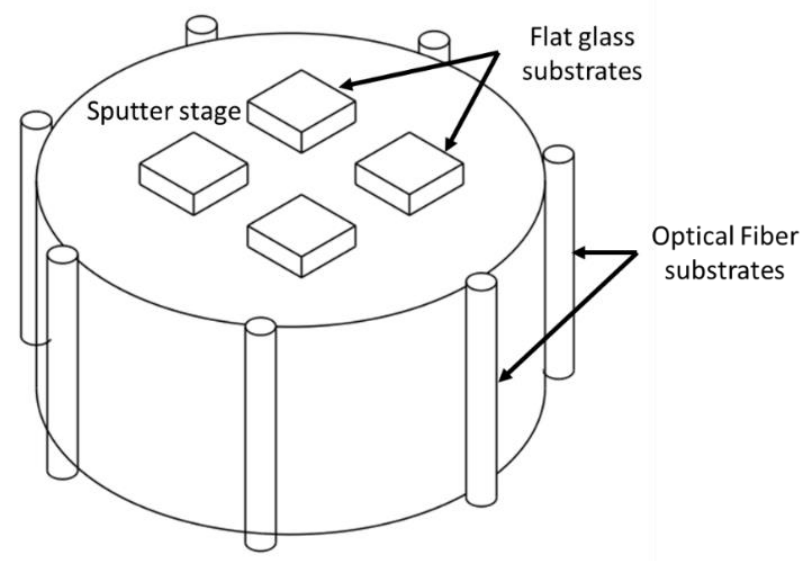

Figure 2-9 Sputter coating of flat glass substrates and optical fiber substrates.

\subsection{Surface topology imaging}

Scanning electron microscopy (SEM) and atomic force microscopy (AFM) was used to evaluate the formation of monolayers and nanopatterns. SEM uses the interaction of electrons with the sample to form images. Electrons interact with the sample and can produce secondary electrons, back-scattered electrons, Auger electrons and characteristic X-rays. The commonly used mode of imaging relies on the generation of secondary electrons due to the ionization of the sample atoms. The secondary electrons are ejected close to the material's surface within a region of few nanometers marking the surface topology of the sample. These electrons are attracted to a detector that has an applied bias. This gives the nanoscale imaging resolution required to study the surface topology [124]. For non-conductive specimens, the bombardment of electrons can result in accumulation of electrostatic charge at the surface. This reduces image quality and requires nonconductive samples to be coated with a thin layer of a conductive material. For SEM images acquired in this thesis JEOL 6380LV scanning electron microscope was used.

Atomic force microscopy is a form of scanning probe microscopy which involves raster scanning a tip over a surface to measure either topology, electronic properties, magnetic properties or mechanical properties [125]. A standard AFM setup uses a sharp tip (a few tens of nanometers) on a cantilever that is attached to a piezoelectric scanner that reveals motion with nanometer precision. The detection of surface roughness is usually a laser that reflects off the cantilever onto a position sensitive photodetector. The tip can be held in the contact mode, non-contact mode, and tapping 
mode for evaluating different types of surfaces. In the contact mode, the tip is in direct contact with the sample and the mapping of the deflection provides the surface topology. In the non-contact mode, the tip hovers over the sample surface and the attractive Van der Waals forces are detected. Since these forces are miniscule, the tip is oscillated, and AC detection methods are used to measure the forces by measuring the amplitude, phase and frequency of the oscillation. The tapping mode is a hybrid of contact mode and non-contact mode where the tip is oscillated and instead of the tip dragging over the sample surface, it taps the surface at high frequency. Since it is not in constant contact with the sample, it is less destructive to the sample while still maintaining the advantages of the contact mode. AFM Nanoscope IIIa (Bruker, USA) was used to characterize NSL surfaces in this thesis and MFP-3D-BIO AFM (Oxford Instruments, UK) was used to characterize NPS surfaces in this thesis.

\subsection{Absorbance measurements}

To characterize the SERS substrates, absorbance measurements were performed. For flat NSL substrates, an integrating sphere-based spectrometer (UV3600, Shimadzu, Japan) setup was used to collect the absorbance spectra.

\subsection{Calculation of SERS enhancements.}

The near field intensity enhancement close to the surface of the nanoparticle is given by

$$
I E F_{\text {surf }}=\left[\frac{E_{\text {surf }}\left(\omega_{\mathrm{exc}}\right)}{E_{0}\left(\omega_{\mathrm{exc}}\right)}\right]^{2}
$$

Where $E_{\text {surf }}$ is the magnitude of the local electric field close to the surface of the nanoparticle, $E_{0}$ is the incident electric field and $\omega_{\text {exc }}$ is the frequency of the incident electric field. The Raman scattered electric field also interacts with the nanoparticle and is enhanced similarly. Hence the total SERS enhancement factor is given by

$$
I E F_{\text {total }}=\left[\frac{E_{\text {surf }}\left(\omega_{\text {exc }}\right)}{E_{0}\left(\omega_{\text {exc }}\right)}\right]^{2} \times\left[\frac{E_{\text {surf }}\left(\omega_{\text {sca }}\right)}{E_{0}\left(\omega_{\text {sca }}\right)}\right]^{2} \approx\left[\frac{E_{\text {surf }}\left(\omega_{\text {exc }}\right)}{E_{0}\left(\omega_{\text {exc }}\right)}\right]^{4}
$$


where $E_{0}\left(\omega_{\text {sca }}\right)$ is the Raman scattered electric field. As a first order approximation for shifts closer to the excitation wavelength, $E_{\text {surf }}\left(\omega_{\text {sca }}\right) \approx E_{\text {surf }}\left(\omega_{\text {exc }}\right)$. This leads to the theoretical $E^{4}$ proportionality of the electromagnetic enhancement of SERS.

The enhancement factors (EF) reported in the literature vary significantly and can be as high as $10^{14}$ [11], [126]. These variations are largely due to the various definitions of EFs. There are various parameters that affect the SERS EF calculations. The first factor is the normalization of the spontaneous Raman and SERS intensities, where they must be normalized to the concentration of the analyte contributing to each effect. Since SERS is a near field effect, the normalization by concentration does not represent the SERS EF accurately. Moreover, the hot-spots are difficult to locate, and bulk normalization might not effectively quantify such variations.

\subsubsection{Analytical enhancement factor}

For a Raman signal intensity $I_{\mathrm{RS}}$ obtained from a specific concentration $c_{R S}$ of analyte and SERS signal intensity $I_{\text {SERS }}$ for a concentration $c_{\text {SERS }}$, the basic definition of analytical SERS enhancement factor is [127]

$$
E F_{\mathrm{SERS}}=\frac{\left(\frac{I_{\mathrm{SERS}}}{c_{\mathrm{SERS}}}\right)}{\left(\frac{I_{\mathrm{RS}}}{c_{\mathrm{RS}}}\right)}
$$

This is a simple analytical expression for calculating enhancement factors for liquid samples under normal and SERS conditions. The assumption is that the intensities scale linearly to the concentrations of analytes. An advantage of this approach is that it can be used for different concentrations in SERS and RS to observe good SNR Raman spectra, a higher concentration is generally required for RS spectra collection. This does not treat SERS as a surface effect and in cases where molecules do not adsorb to the nanoparticles strongly, this can result in erroneous enhancement factors [127].

\subsubsection{Substrate enhancement factor}

The analytical enhancement factor has limitation as it does not account for the number of molecules adsorbed to the surface of nanoparticles and can result in erroneous EF values. When 
substrates are used with immobilized nanoparticles or nano-patterns, it is important to normalize the intensities with number of molecules. For standard Raman measurement, the number of molecules, $N_{v o l}$, are computed by analyzing the excitation volume of the laser. For SERS, it is assumed that using experimental conditions, a monolayer of molecules, $N_{\text {surf }}$, are adsorbed to the nanoparticle surface. The Substrate SERS (SSERS) enhancement factor is defined as [127]

$$
E F_{\mathrm{SSERS}}=\frac{\left(\frac{I_{\mathrm{SERS}}}{N_{\text {surf }}}\right)}{\left(\frac{I_{\mathrm{RS}}}{N_{\mathrm{vol}}}\right)}=\frac{I_{\text {SERS }}}{I_{\mathrm{RS}}} \cdot \frac{N_{\mathrm{vol}}}{N_{\text {surf }}}
$$

Intensity enhancements can be decoupled from the molecular ratios. The estimation of molecular ratio is susceptible to errors since the exact focal volume needs to be calculated and the number of adsorbed molecules on the surface of the nanoparticle need to be accurately estimated which is challenging.

\subsubsection{Experimental enhancement factors}

For flat glass substrates, a $10 \mu \mathrm{L}$ of Crystal Violet (CV) droplet was deposited on the substrate and the spectra, $I_{\mathrm{RS}}^{\text {flat }}$, was measured immediately after depositon. The spectra, $I_{\mathrm{SERS}}^{\mathrm{flat}}$, from fabricated flat SERS substrates were acquired after incubation of the substrates in CV dye for 3 hours. The intensity enhancement factor $e_{I}^{\text {flat }}$ was defined as

$$
e_{\mathrm{I}}^{\mathrm{flat}}=\frac{\mathrm{I}_{\mathrm{SERS}}^{\mathrm{flat}}}{\mathrm{I}_{\mathrm{RS}}^{\text {flat }}}
$$

The experimentally obtained enhancement factors (intensity enhancement factor $e_{\mathrm{I}}^{\text {flat }}$ ) were separated from the molecular ratio. The molecular contribution ratio can be calculated using the number of molecules $\left(N_{\mathrm{vol}}\right)$ for RS contained in the focal volume and the number of molecules adsorbed on the surface ( $N_{\text {surf }}$ ) of the nanoparticle for SERS. The estimation of $N_{\text {vol }}$ depends on the careful characterization of excitation volume and $N_{\text {surf }}$ on careful estimation of the number of molecules adsorbed on the surface of the nanoparticle. Estimation of $N_{\text {surf }}$ is quite challenging as studies have found that only $0.01 \%$ of the adsorbed molecules on the surface contribute to SERS [128]. Intensity enhancement factors provide a practical way of calculating enhancement factors 
and since all the experiments performed in this study were done using the same instrumentation and SERS incubation protocols, the IEF can be used for comparison.

For fiber substrates, the non-patterned fiber substrates were dipped in CV solution and Raman spectra were collected immediately $\left(I_{\mathrm{RS}}^{\mathrm{fiber}}\right)$. The SERS fiber substrates were incubated in the CV solution for 3 hours following which, SERS spectra (I fiber $)$ were collected. The intensity enhancement factor $e_{\mathrm{I}}^{\text {fiber }}$ was defined as:

$$
e_{\mathrm{I}}^{\mathrm{fiber}}=\frac{\mathrm{I}_{\mathrm{SERS}}^{\text {fiber }}}{\mathrm{I}_{\mathrm{RS}}^{\text {fiber }}}
$$

\subsection{Theoretical modeling}

\subsubsection{COMSOL Multiphysics}

COMSOL is a modular software package that performs multiphysics simulations by coupling various physics modules. For modeling the interaction of electromagnetic (EM) with nanomaterials at optical frequencies (nanometer to micrometer wavelengths), the wave optics module of COMSOL is utilized. The module supports simulations for optical fibers, photonic waveguides, photonic crystal, non-linear optics etc. in 2D and 3D environments.

Macroscopically, Maxwell's equations govern the EM analysis with appropriate boundary conditions. The equations that form the base of EM analysis are defined as:

$$
\begin{gathered}
\nabla \times \mathbf{H}=\mathbf{J}+\frac{\partial \mathbf{D}}{\partial t} \\
\nabla \times \mathbf{E}=-\frac{\partial \mathbf{B}}{\partial t} \\
\nabla \cdot \mathbf{D}=\rho \\
\nabla \cdot \mathbf{B}=0
\end{gathered}
$$


Where $\mathbf{E}$, is the electric field intensity $\left(\mathrm{V} \cdot \mathrm{m}^{-1}\right), \mathbf{H}$ is the magnetic field intensity $\left(\mathrm{A} \cdot \mathrm{m}^{-1}\right), \mathbf{J}$ is the current density $\left(A \cdot \mathrm{m}^{-2}\right), \mathbf{D}$ is the electric displacement or electric flux density $\left(\mathrm{C} \cdot \mathrm{m}^{-2}\right), \mathbf{B}$ is the magnetic flux density $\left(\mathrm{Wb} \cdot \mathrm{m}^{-2}\right)$, and $\rho$ is the electric charge density $\left(\mathrm{C} \cdot \mathrm{m}^{-3}\right)$.

Along with the above equations, the equation of continuity is used to form

$$
\nabla \cdot \mathbf{J}=-\frac{\partial \rho}{\partial t}
$$

These equations link the fields, charges, and current densities. To explain the interaction of matter with the fields, we need to use the following constitutive relations

$$
\begin{gathered}
\mathbf{D}=\epsilon_{0} \epsilon_{r} \mathbf{E} \\
\mathbf{B}=\mu_{0} \mu_{r} \mathbf{H} \\
\mathbf{J}=\sigma \mathbf{E}
\end{gathered}
$$

Where $\epsilon_{0}, \mu_{0}$ are the electric permittivity and magnetic permeability of free space respectively, and $\epsilon_{r}, \mu_{r}$, J and $\sigma$ denote the relative permittivity, relative permeability, current density and conductivity respectively.

\subsubsection{Frequency domain formulation for the total electric field and scattered electric field}

Scattered field intensity can be an interesting quantity for many problems. In such cases, the incident electric field is known, and only scattered field needs to be obtained. Since the scattered field can be much lower in magnitude than the incident electric field, solving only for scattered field can improve the accuracy of the simulation [129].

The frequency domain equation can be written as [130]:

$$
\nabla \times\left(\mu_{r}^{-1} \nabla \times\left(\boldsymbol{E}_{\mathrm{tot}}\right)\right)-k_{0}^{2} \varepsilon_{\mathrm{r}}\left(\boldsymbol{E}_{\mathrm{tot}}\right)=0
$$

where the wave number of free space is $k_{0}=\frac{\omega}{c_{0}}$, the total electric field is $\boldsymbol{E}_{\text {tot }}$. 
For a known incident plane wave traveling in the positive $x$ direction, with the polarization of electric field along the $\mathrm{z}$ axis, the scattered field formulation is achieved by replacing $\boldsymbol{E}_{\text {tot }}$ with $\boldsymbol{E}_{\text {inc }}+\boldsymbol{E}_{\text {sca }}$ where the scattered electric field $\boldsymbol{E}_{\text {sca }}$ is the dependent variable and the incident electric field $\boldsymbol{E}_{\text {inc }}$ is given by

$$
\boldsymbol{E}_{\mathrm{inc}}=E_{o} e_{0}^{-j k x} \hat{\mathbf{z}}
$$

where $E_{0}=1 \mathrm{~V} \cdot \mathrm{m}^{-1}$.

\subsubsection{Boundary conditions}

The boundary conditions imposed at the interface between two media are given by

$$
\begin{aligned}
& \widehat{\mathbf{n}} \times\left(\mathbf{E}_{\mathbf{1}}-\mathbf{E}_{\mathbf{2}}\right)=0 \\
& \widehat{\mathbf{n}} \cdot\left(\mathbf{D}_{\mathbf{1}}-\mathbf{D}_{\mathbf{2}}\right)=\rho_{\mathrm{s}} \\
& \widehat{\mathbf{n}} \times\left(\mathbf{H}_{\mathbf{1}}-\mathbf{H}_{\mathbf{2}}\right)=\mathbf{J}_{\mathrm{s}} \\
& \widehat{\mathbf{n}} \cdot\left(\mathbf{B}_{\mathbf{1}}-\mathbf{B}_{\mathbf{2}}\right)=0
\end{aligned}
$$

where $\rho_{\mathrm{s}}$ is the surface charge density $\left(\mathrm{C} \cdot \mathrm{m}^{-2}\right)$ and $\mathbf{J}_{\mathrm{s}}$ is the surface current density $\left(\mathrm{A} \cdot \mathrm{m}^{-1}\right)$

For truncating external boundaries of the computational domain, the following boundary conditions are defined:

- Perfect electric conductor (PEC) to model lossless metallic surface or as a symmetry type boundary condition given by

$$
\widehat{\mathbf{n}} \times \mathbf{E}=0
$$

- Perfect magnetic conductor (PMC) to model high surface impedance or a symmetry type boundary condition by

$$
\widehat{\mathbf{n}} \times \mathbf{H}=0
$$


- Perfectly matched layers are special boundary truncation domains which work by absorbing any fields incident on them. Mathematically, PML is a domain that comprises of anisotropic and complex valued permittivity and permeability.

- Floquet-Bloch periodic boundary conditions

PEC and PMC boundaries can be used as symmetric boundary conditions parallel and perpendicular to linearly polarized E-field and H-fields. In cases where boundaries are cannot be parallel or perpendicular to the applied E-field polarization, other boundary conditions such as Floquet-Bloch condition must be applied.

The solution of the wave equation in periodic media can be obtained using the FloquetBloch theorem. Bloch's theorem generally describes electrons in crystals, but it can be readily extended to evaluation of particles in periodic media (for e.g. photonic crystals, arrays of nanoparticles etc). The periodic boundary conditions state that the electric field in the unit cell is related to the adjacent cells by a complex constant.

In all the simulations, the optical properties of gold were used from the built-in library of COMSOL Multiphysics software package. The optical properties used were taken from the study performed by Rakić et. al [131] and are shown in Figure 2-10.

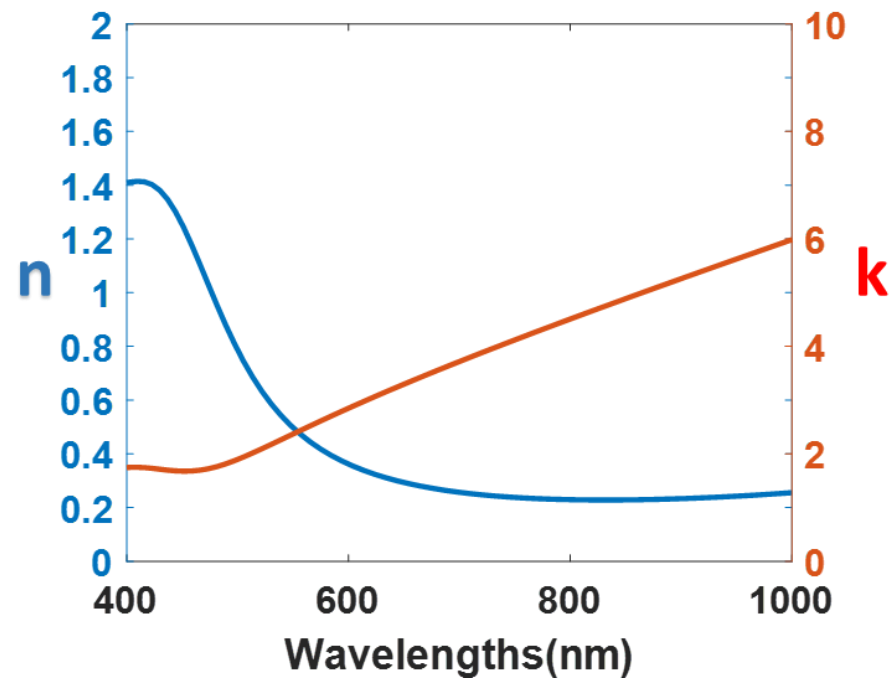

Figure 2-10 Complex refractive index (n-real part, k-imaginary part, unitless) of gold used in the study. 


\subsubsection{Scattering, absorption and extinction cross sections}

For absorption cross section which is defined as the ratio of the absorbed power to the irradiance [132], the resistive heating computed by COMSOL can be used:

$$
\sigma_{\mathrm{abs}}=\frac{\iiint Q_{\mathrm{rh}} d V}{I_{0}}
$$

where $\sigma_{\mathrm{abs}}$ is the absorption cross section, $Q_{\mathrm{rh}}$ is resistive heating (due to the absorbed power) computed by COMSOL within the volume of interest and $I_{0}$ is the incident intensity.

Scattering cross section is defined as the ratio the scattered power to the irradiance [25]. In COMSOL, the scattered power is computed by integrating the Poynting vector over the surface bound by the nanoparticle.

$$
\sigma_{\mathrm{sca}}=\frac{\frac{1}{2} \iint \operatorname{Re}\left[\boldsymbol{E}_{\mathrm{sca}} \times \boldsymbol{H}_{\mathrm{sca}}^{*}\right] d S}{I_{0}}
$$

The extinction cross section is given by [132]:

$$
\sigma_{\text {ext }}=\sigma_{\text {sca }}+\sigma_{\text {abs }}
$$

\subsubsection{NSL substrates}

Curved nanotriangles were formed because of the PS sphere mask used in NSL. A tightly packed monolayer of PS spheres creates gaps as depicted in Figure 2-11. To form the curved nanotriangles, the following steps are performed in Inventor (Autodesk, USA) CAD software: 1) A sketch was created using $1 \mu \mathrm{m}$ diameter spheres arranged in a tightly packed geometry. A rectangle was created, and a Boolean difference operation is performed to get a curved nanotriangle. 2) A filet operation was performed on the nanotriangle edges to smooth out the sharp points which can cause singularity in theoretical simulations. 3) The rounded nanotriangle was extruded to the required height and another filet operation was performed to create rounded edges to obtain the 3D nanotriangle as depicted in Figure 2-11. 
A

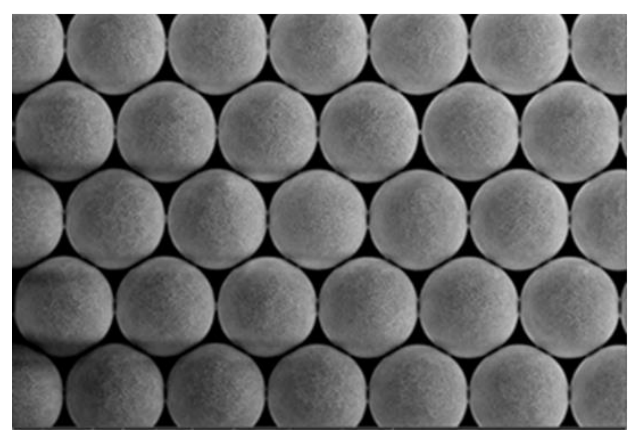

B

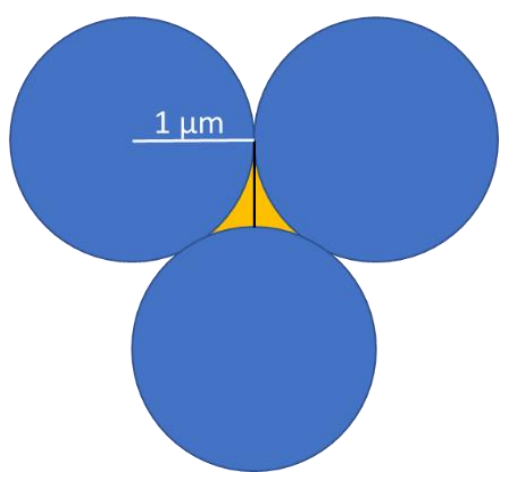

C

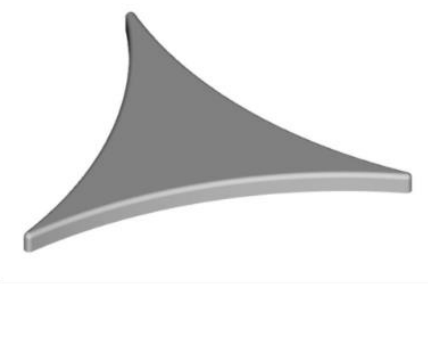

Figure 2-11 A) SEM image of a monolayer of PS spheres B) A boolean difference operation is performed on a flat rectangle to create a curved nanotriangle. C) The flat sketch is extruded to appropriate heights to create a 3D nanotriangle and filet operation is performed on the edges to create smooth surfaces.

Table 2-3 Physical description of the nanotriangles

\begin{tabular}{cc} 
Quantity & Value \\
\hline Circumference & $1.184 \mu \mathrm{m}$ (base) $1.153 \mu \mathrm{m}$ (top) \\
Surface area & $0.06038 \mu \mathrm{m}^{2}$ \\
Volume & $7.807 \times 10^{-4} \mu \mathrm{m}^{3}$
\end{tabular}

The nanotriangle was imported in COMSOL (physical dimensions provided in Table 2-3) and a frequency domain analysis was performed using the wave optics module of COMSOL. A twostep simulation was set-up as follows:

1) The total field formulation of COMSOL was used to compute the incident electric field for the air and glass interface which was extended infinitely in the $\mathrm{X}$ and $\mathrm{Y}$ directions through periodic boundary conditions and a port was used to define the excitation where the polarization of electric field was in the $\mathrm{Y}$ direction and the propagation of wave was in the $\mathrm{Z}$ direction as shows in Figure 2-12. For an air-glass interface, the total electric field in air is a superposition of $\boldsymbol{E}_{\text {inc }}$ and $\boldsymbol{E}_{\text {ref }}$ while in the total electric field in glass is given by $\boldsymbol{E}_{\text {trans }}$. To compute the scattering of a nanoparticle, the total electric field incident onto the nanoparticle should be known to use the scattered field formulation in COMSOL. The analytical expression of the incident electric field becomes complicated when there is an interface involved [133]. Hence, this approach is followed to compute the superposition of 
incident and reflected waves in the free space domain and transmitted wave in the substrate. The resultant computed electric field is used as an input for the next computation step.

2) This computed electric field in the previous step is used as a known incident electric field in this second step where a nanoparticle is introduced at the boundary of free space and glass substrate and the scattered field is computed for the nanoparticle. In this case, the simulation boundary is truncated using perfectly matched layer (see sec. 2.9.2) boundaries on all sides to assist in absorbing any electric fields incident on the boundary.

A

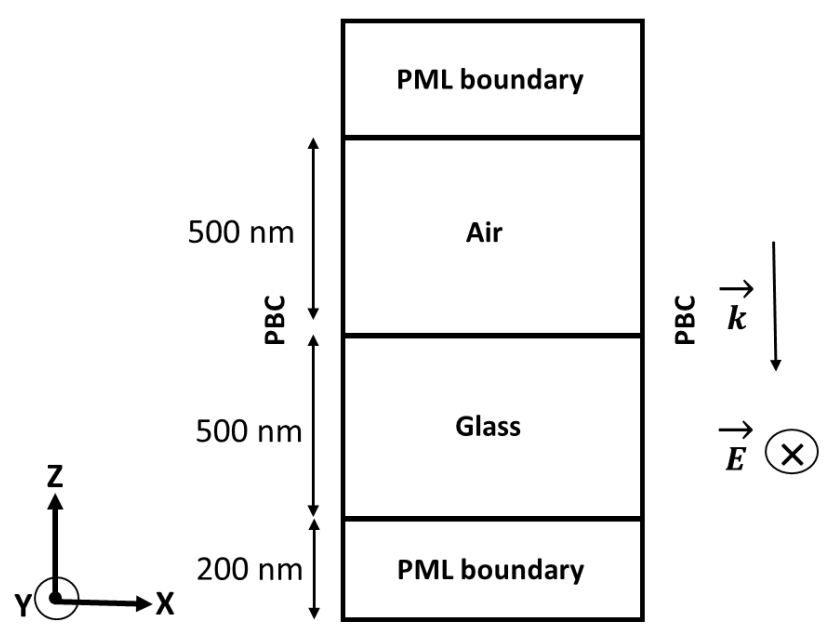

B

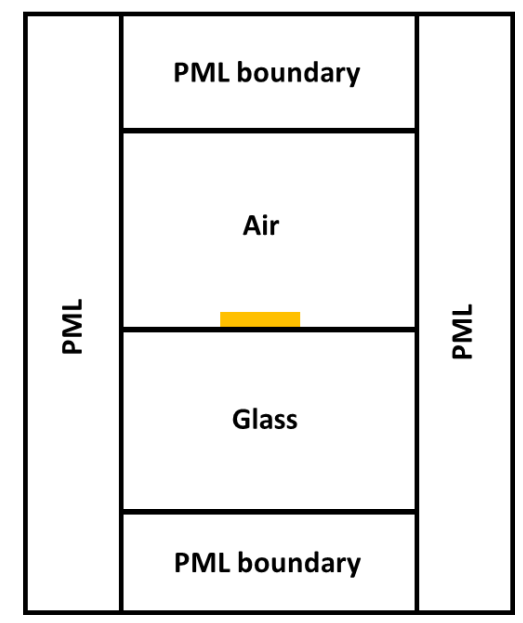

Figure 2-12 Schematic of computational domain created for calculating the electromagnetic enhancement of nanotriangles. 1) Periodic boundary conditions (PBC) are applied in the $\mathrm{X}$ and $\mathrm{Y}$ (hidden in this view) for computing background electric field. 2) Perfectly matched layer (PML) boundaries are used to truncate the domain in each direction.

The enhancement of the electric field is of main interest for SERS. Since the SERS enhancement factor is proportional to $\frac{\left|E_{\text {surf }}\right|^{4}}{\left|E_{0}\right|^{4}}$, the basic electromagnetic (EM) enhancement computed was defined as

$$
e_{\mathrm{NSL}}^{\mathrm{comp}}=\frac{\left|\boldsymbol{E}_{\text {surf }}\right|^{4}}{\left|\boldsymbol{E}_{\mathrm{inc}}\right|^{4}}
$$

Where $\boldsymbol{E}_{\text {surf }}$ is the electric field computed at the surface of the nanoparticle and $\boldsymbol{E}_{\text {inc }}$ is the incident electric field. Maximum and integrated enhancement factors were defined as shown in Table 2-4. 
Table 2-4 Definition of enhancement factors.

\begin{tabular}{|c|c|c|}
\hline Enhancement Factor & Definition & Notes \\
\hline Max & $\max \left[e_{\mathrm{NSL}}^{\mathrm{comp}}\right]$ & $\begin{array}{l}\text { Surface area defined by the top face of } \\
\text { the nanoparticle at the boundary of air } \\
\text { \& nanoparticle }\end{array}$ \\
\hline Integrated & $\oint e_{\mathrm{NSL}}^{\mathrm{comp}} d s$ & $\begin{array}{l}\text { Integrated over the same surface as } \\
\text { defined for the max operation. }\end{array}$ \\
\hline
\end{tabular}

To simulate a more realistic geometry, a hexagonal array of nanotriangles was utilized to create a periodic simulation geometry as shown in Figure 2-13. The simulation geometry was bound by Floquet boundary conditions in the X-Y directions and PML boundaries were used to truncate the $\mathrm{Z}$ direction as shown in Figure 2-14. A similar two step approach was utilized for the periodic array simulations to obtain the $\vec{E}$ field enhancements

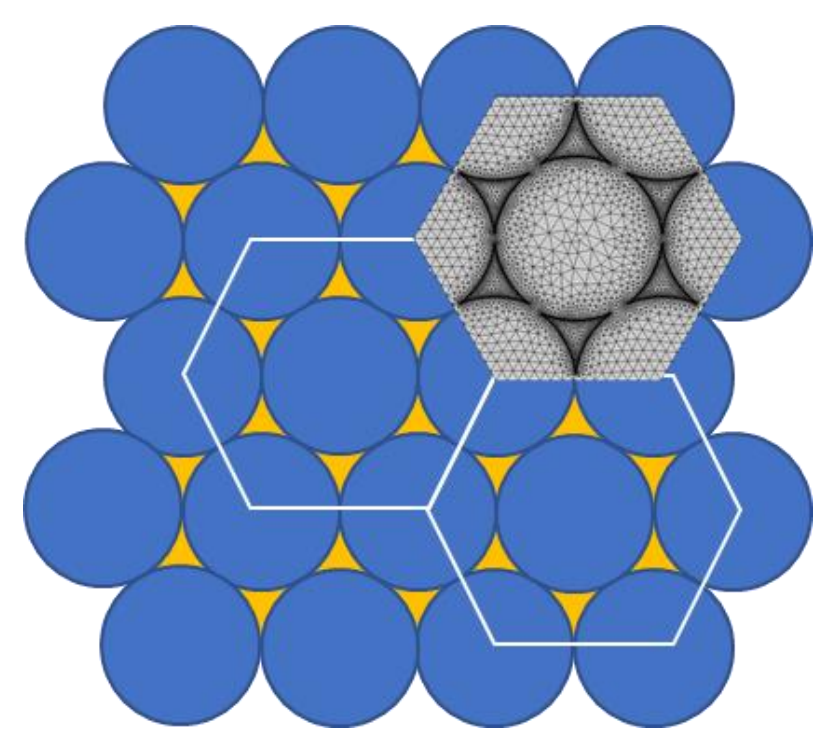

Figure 2-13 Hexagonal periodic arrangement of the nanotriangles with insets showing unit cell. 

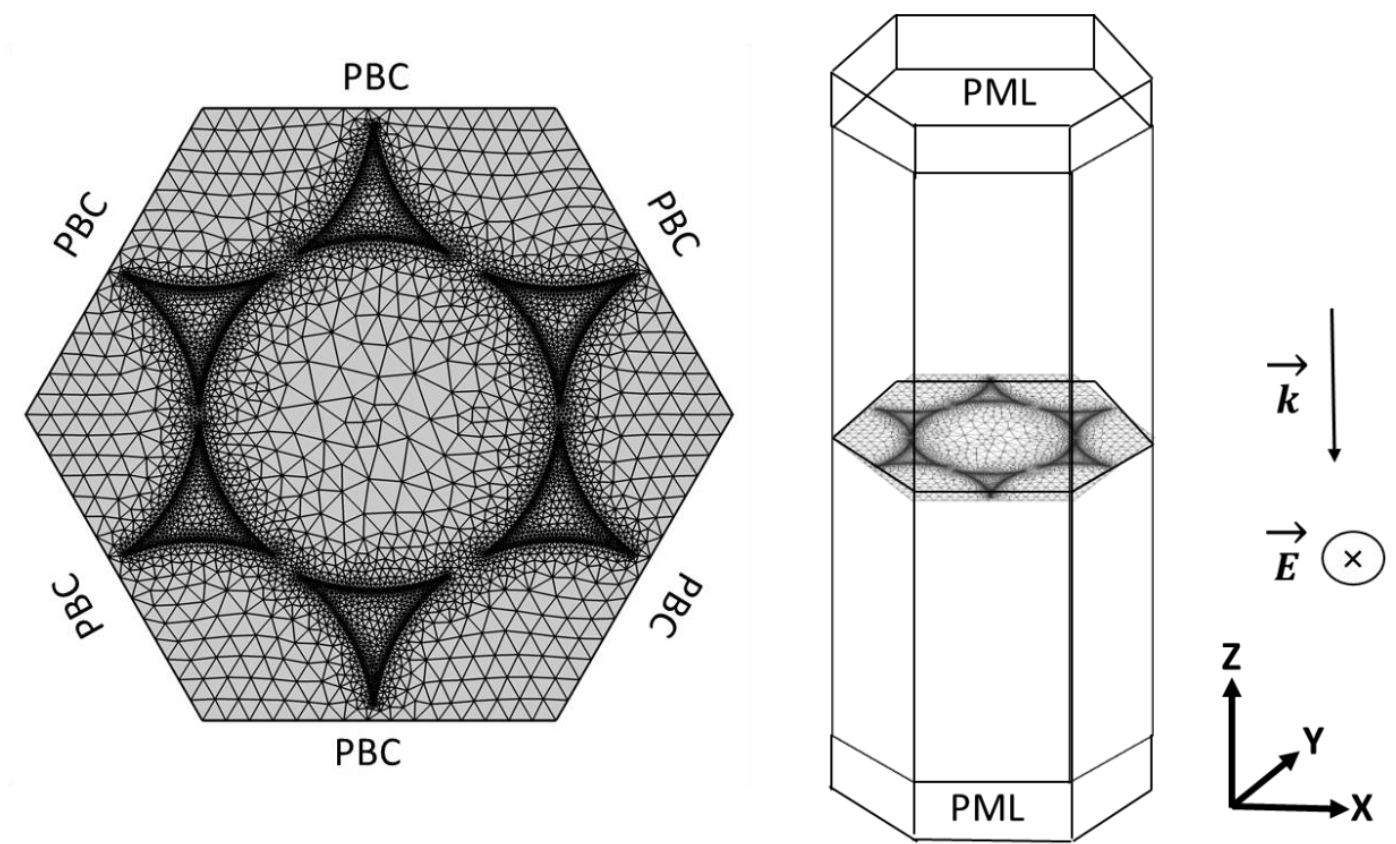

Figure 2-14 Computational setup in for periodic simulation of the hexagonal periodic cell.

\subsubsection{Nanoporous substrates}

Gold coated nanoporous surface of the glass was modeled as a nanoporous film with varying thickness for simplicity. The underlying glass also had a nanoporous structure and creating a 3D nanoporous structure to represent the underlying glass as well as the top layer required a lot of computational resources. Thus, only the gold nanoporous film was modelled surrounded by air. The nanoporous glass pattern obtained using AFM was used to create a geometry mimicking the nanoporous structure. The nanoporous structure obtained using AFM was converted to a binary image using median as a threshold. The binarization resulted in edge elements having a pore geometry that was abruptly terminated due to the edges. The edge elements of the digitized nanoporous structures were removed due to their extremely small size and sharp cut edges which are not representative of the actual nanostructure as shown in Figure 2-15.

PEC and PMC boundary conditions were placed orthogonally in the $X$ and $Y$ directions while PML layers were used to truncate the $\mathrm{Z}$ direction. The values of the electric field obtained through theoretical simulations was integrated for the simulation volume near the metal layer. The resulting integrated electric field was normalized by the integrated volume for comparison between NS-7 and NS-17 substrates. 
Since the image quality obtained from AFM for the NS-7 substrates was not suitable for creating a CAD geometry, the 3D geometry of NS-17 substrates created as described previously was used to create a nanoporous structure emulating the NS-7 substrates by scaling the size and density of the pores to match those measured for the NS-7 substrates. 

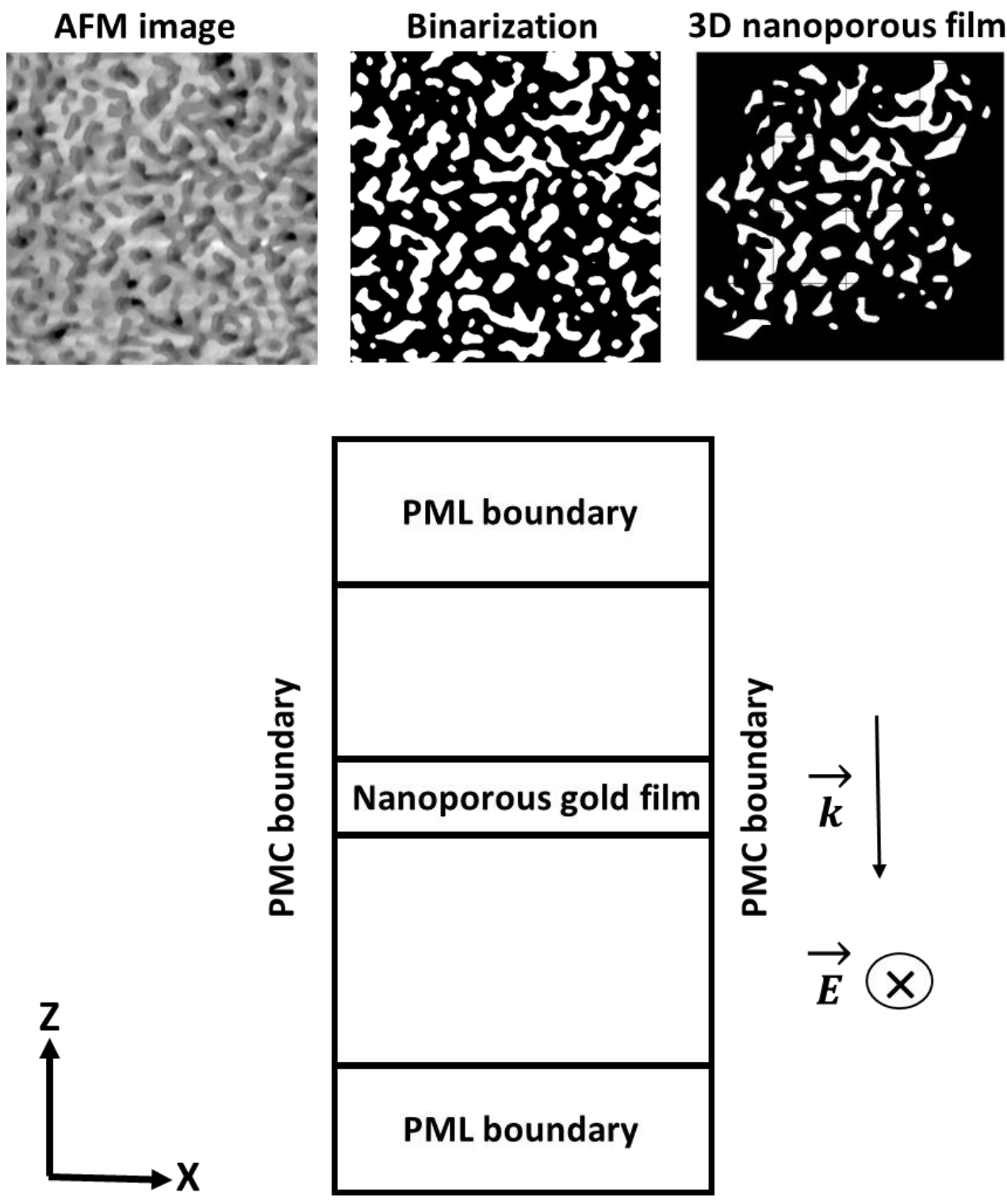

Figure 2-15 3D model of nanoporous film and computational domain for modeling nanoporous structures. The top row shows the binarization of the topographical image. The edge elements are truncated due to the field of view and are omitted from the 3D modeling. 


\section{Results and Discussion}

\subsection{NSL substrates}

Two types of NSL substrates were created 1) Flat NSL substrates and 2) Fiber-optic NSL substrates.

\subsubsection{Flat NSL substrates}

To perform NSL, drop coating method and air-water interface method was used for creating monolayers of PS spheres.

\subsubsection{Drop coating method}

The simplest fabrication technique for NSL is the drop coating technique. A drop of PS spheres resulted in a thick outer rim which consisted of clumped PS spheres. The thickness changed during multiple experimental trials with same protocols and this was attributed to the variations of the ambient environmental conditions such as humidity and temperature. A monodisperse PS solution created a closely packed hexagonal monolayer and after the removal of the nanospheres, the AFM image of the resultant nanopattern as shown in Figure 3-1. This monolayer was hard to achieve with the drop coating of glasses. The surface of glass, the environmental conditions and the type of PS spheres greatly influenced the fabrication process. Although the method is simple, it does not result in reproducible monolayers. Moreover, such a technique is difficult to use on small fiberoptic tips. 

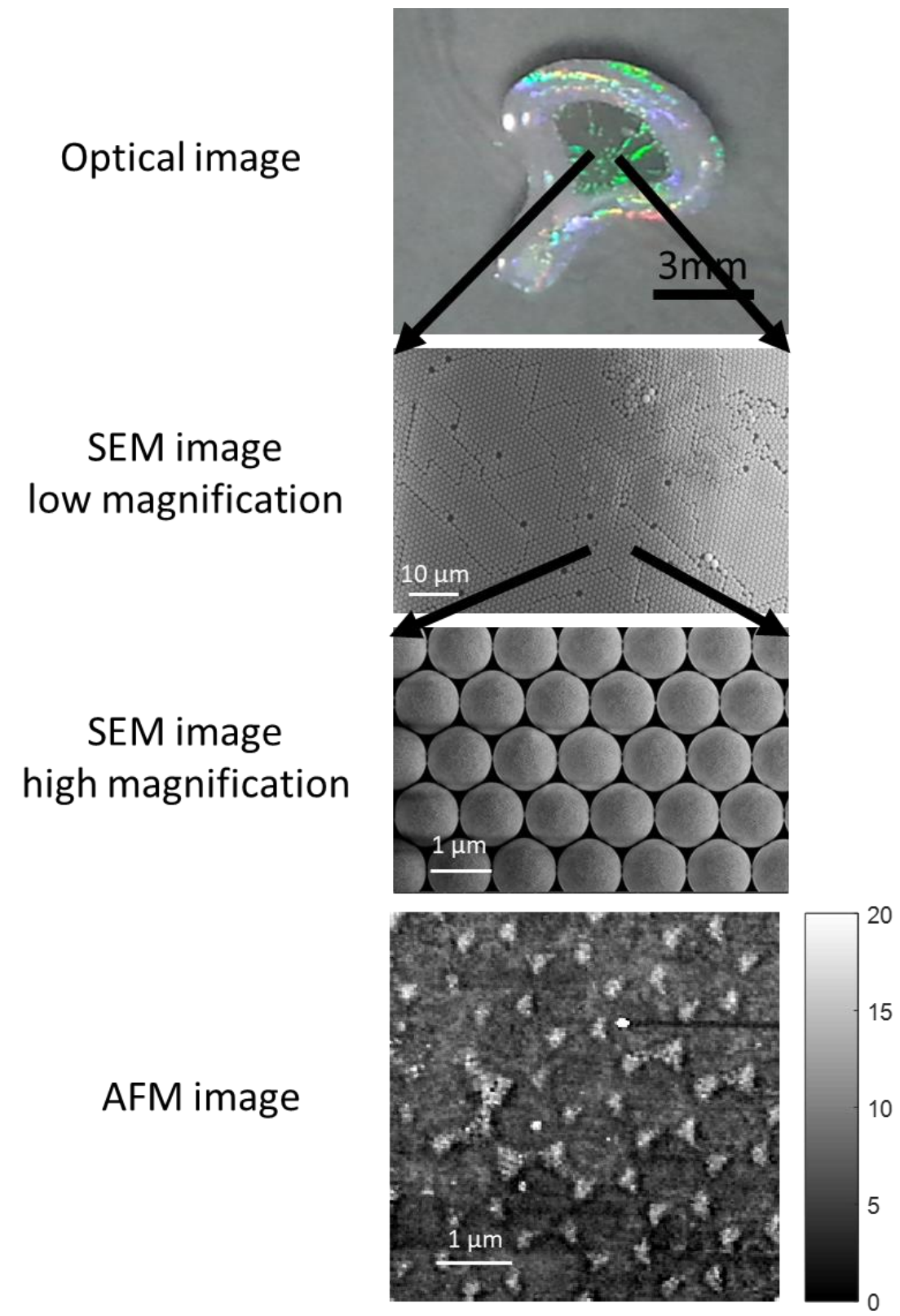

Figure 3-1 Results of the drop coating technique. 1) Optical image of the drop coat showing central monolayer and thicker edges showing a larger amount of PS spheres. 2) SEM images of low and high magnifications of the monolayer. 3) AFM image of the corresponding nanopattern formed after removal of spheres.

\subsubsection{Air-water interface method}

The air water interface method of fabricating monolayers was found to be more optimal than the drop coating method for creating large area monolayers. A fabrication setup is shown in Figure 3-2. Custom Teflon tank was utilized to trap the monolayers at the air water interface to decrease 
the adhesion of the monolayer to the walls. Ethanol used in the dispersion solution assisted in the assembly of monolayers on the air water interface as its density is less than that of water and ethanol molecules tend to cover the surface. The hydrophobic PS spheres get trapped at the interface potential energy well and form a monolayer on the surface of water. The slow dispersion of PS spheres created a gradual filling of the water surface and saturation at the surface was observed when adding further dispersion solution results in dissolution of the sphere in water rather than spreading outwards. Some of the spheres dissolved in water but most of them remained on the surface and an iridescent pattern was seen on the water surface. The glass slides were immersed in the water solution before the dispersion of particles allowing particles to adhere to the surface of the glass. Air-water interface method provided a consistent method for creating monolayers. Larger coverage was possible with this method and by moving the tank, monolayers could be manipulated after they were formed.
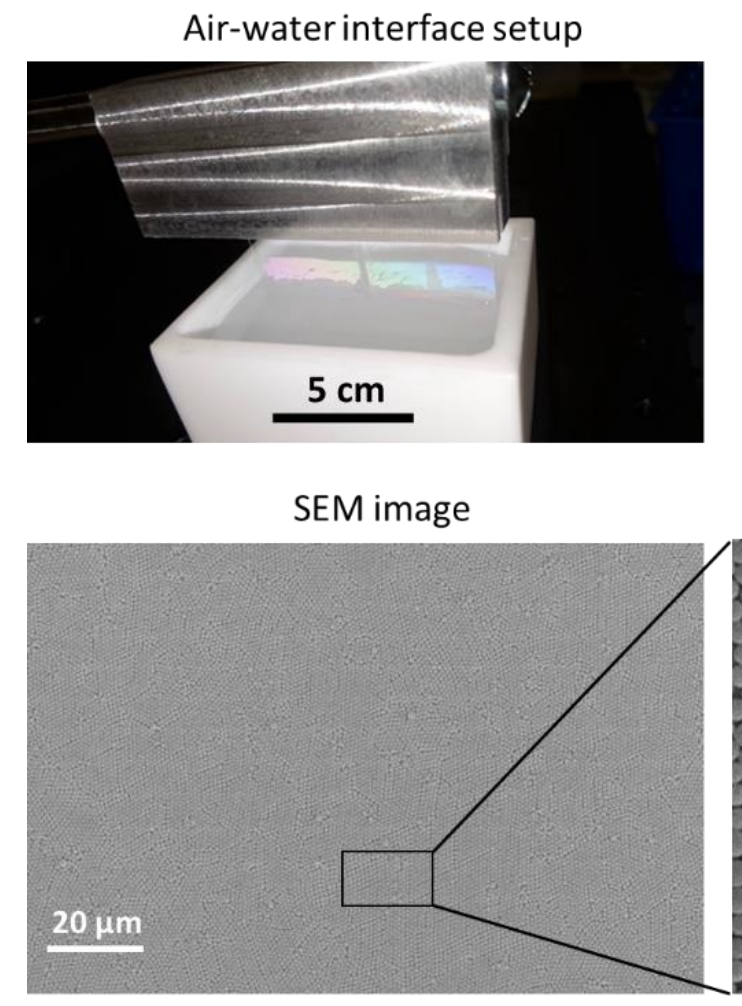

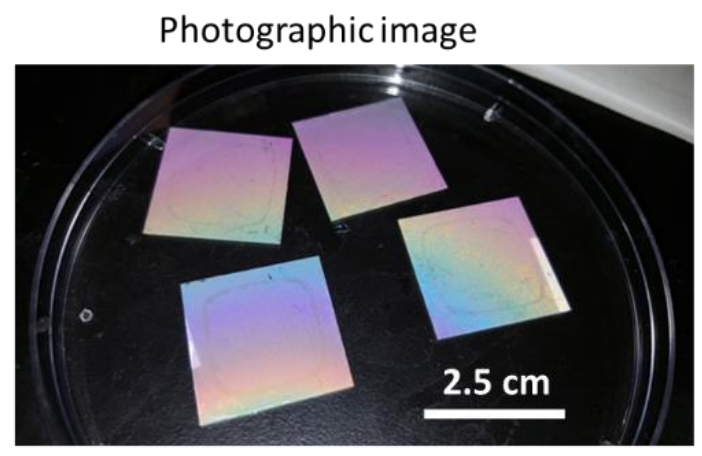

Magnified SEM with AFM image inset

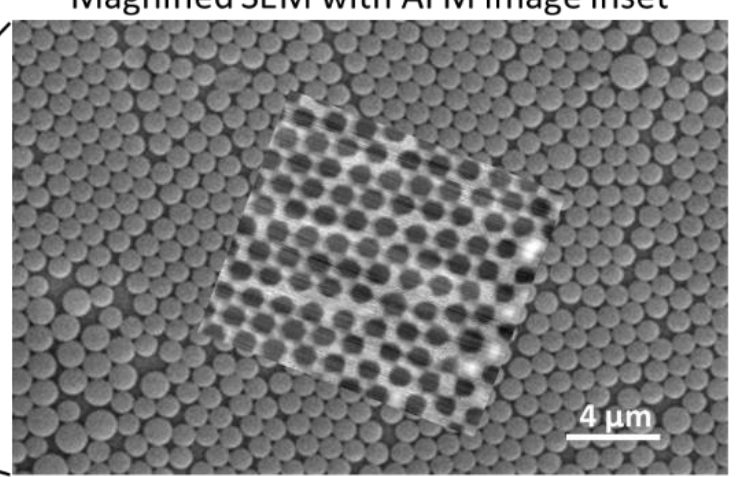

Figure 3-2 Fabrication process for the air-water interface method 1) The setup depicting the dipper assembly used to lower the glass slides in the Teflon tank. 2) A photographic image of the iridescence observed due to the monolayer on the surface of glass. 3) SEM image of the resultant monolayer over a large surface area and 4) AFM image inset of the resultant nanopattern obtained overlaid on top of the magnified SEM image in the center. 


\subsubsection{AFM height analysis}

Following the fabrication of substrates using the air-water interface, Au was sputtered onto the PS monolayer mask. Different times of sputter coating were used to create different thicknesses of the gold nanoparticles. The sputter rate was $2 \AA / s$ of Au layer thickness and the resultant distribution of heights is shown in Figure 3-3. The images show variations in the nanopatterns due to the variability of the PS spheres on the surface. An automated plunger assembly such as Langmuir Blodgett trough setup can be used to further improve the uniformity of the coating. To estimate the mean height of the nanoparticles and to filter out areas devoid of nanoparticles, a mean value was obtained for all the values above the median.

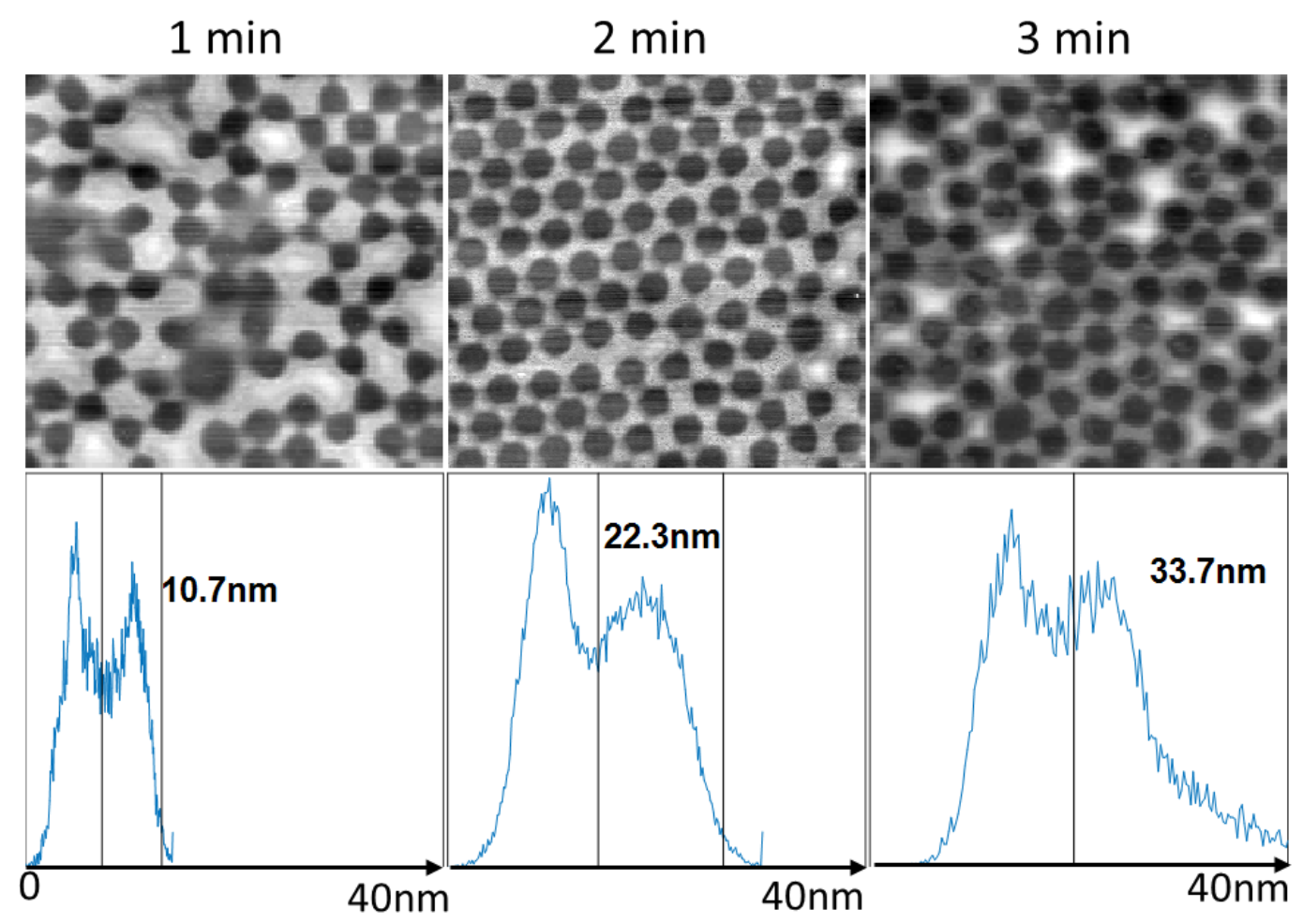

Figure 3-3 Heights of nanoparticles obtained from AFM for 1min, 2min and 3min sputter coating of Au. Vertical lines in the height distributions represent $50^{\text {th }}$ percentile and $99^{\text {th }}$ percentile of heights and the values represent the mean value of heights over $50^{\text {th }}$ percentile. 


\subsubsection{SERS measurements}

SERS spectra were obtained by moving the sample into the best focus location by translating the sample until the spectral intensity was maximized. Since the system used for SERS detection was not confocal, the collection of spectra was insensitive to the minor variation of sample movement while being scanned in lateral direction. Figure 3-4 shows the spectrum for Crystal Violet dye using spontaneous Raman ( $\mathrm{SpR}$ ) and SERS modes without any background correction. The blue spectrum/left axis represents raw $\mathrm{SpR}$ data and the orange spectrum/right axis represents raw SERS data. The broader background peak from $1250 \mathrm{~cm}^{-1}-1750 \mathrm{~cm}^{-1}$ is due to the fluorescence generated by the glass substrate. For $\mathrm{SpR}$, a drop of $\mathrm{CV}$ dye was deposited on the glass substrate and data was collected immediately. The integration time for SERS was 2s and for SpR was 20s. The spectra were normalized by the integration times and are depicted on dual scales for better visualization of SpR peaks.

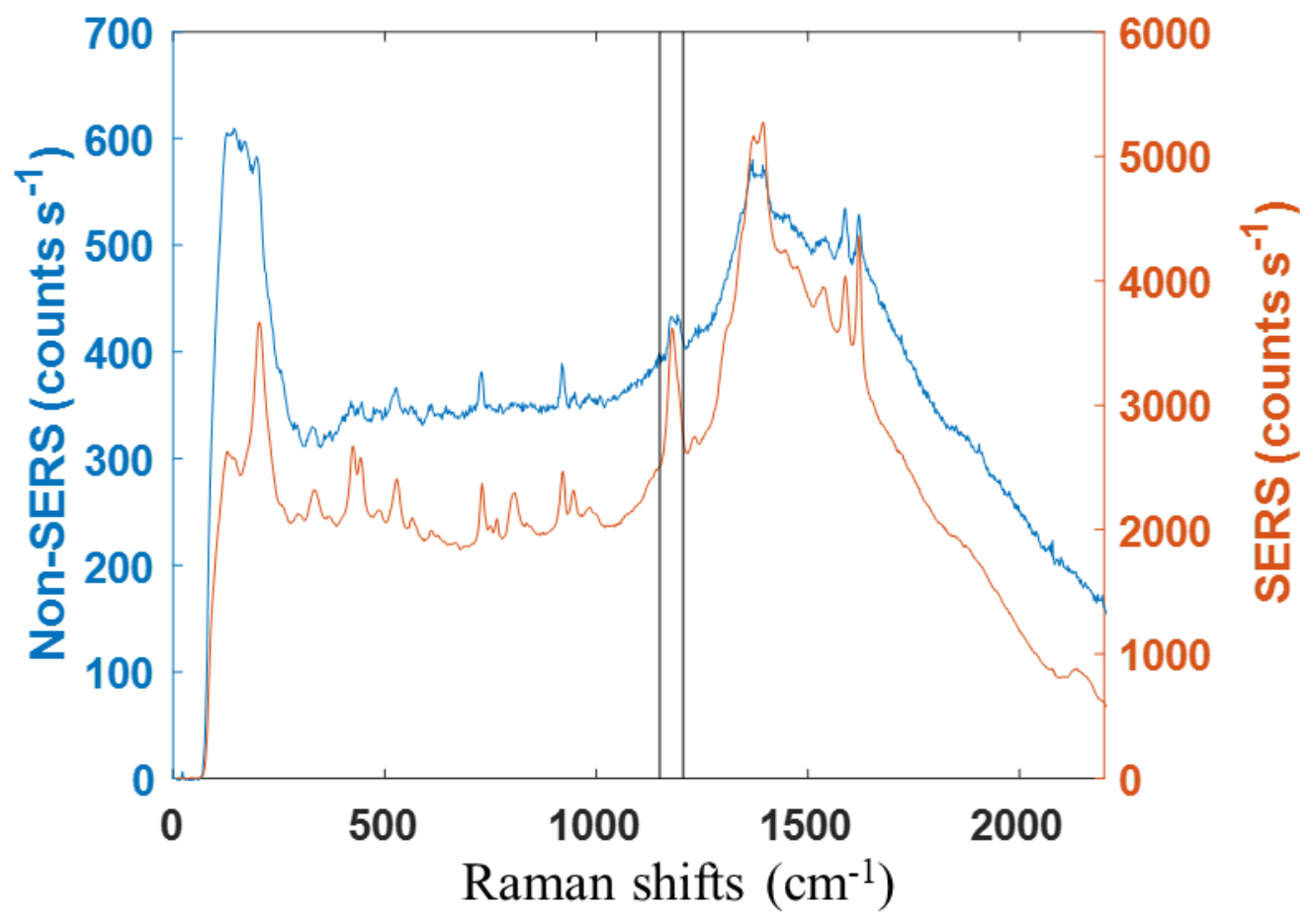

Figure 3-4 Spectra comparison for spontaneous Raman and SERS collection modes for 100PPM CV dye.

A 20nm layer of Au was coated onto a glass slide as a reference. An NSL fabricated glass slide was used with the nonpatterned glass slide and immersed in a solution of 100PPM CV dye for a 
comparison. The spectra obtained from the patterned and nonpatterned substrates are presented in Figure 3-5. The peak assignments of CV dye were obtained from the literature [134]. The peaks obtained from the literature were collected under resonance conditions. SERS spectra could only be obtained from the patterned substrates.

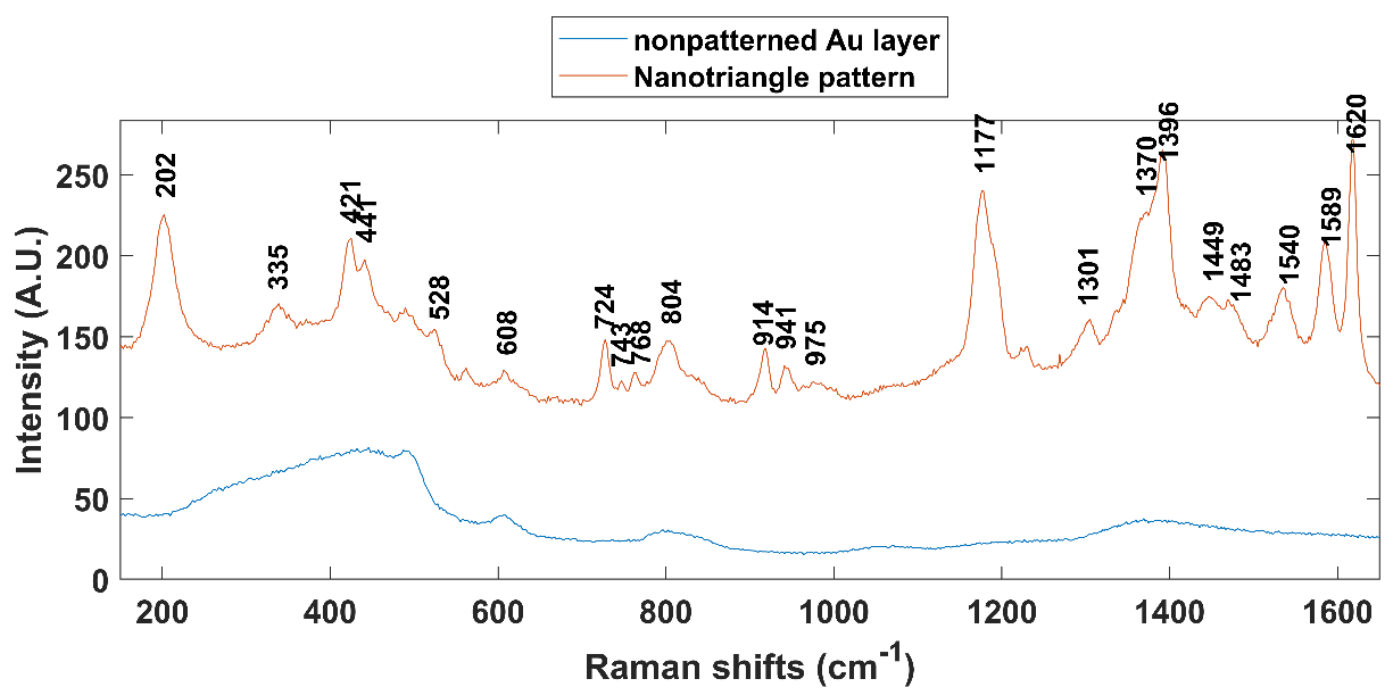

Figure 3-5 Comparison of SERS spectra from patterned and nonpatterned substrates.

\section{Dependence of SERS activity on sputter time}

All SERS spectra presented below are processed with background suppression described in 2.2.2. SERS spectra for different heights of nanoparticles are shown in Figure 3-6. Using the strong Raman peak located at $1177 \mathrm{~cm}^{-1}$, a sputter time of 2 minutes was found to be appropriate for generating SERS which corresponds to a nanoparticle height range of $22 \mathrm{~nm}$ as per the definition of heights mentioned in section AFM height analysis 3.1.1.3. Lower sputter time resulted in lower enhancements while increasing sputtering time increases cost hence 2 min was found to be suitable. 


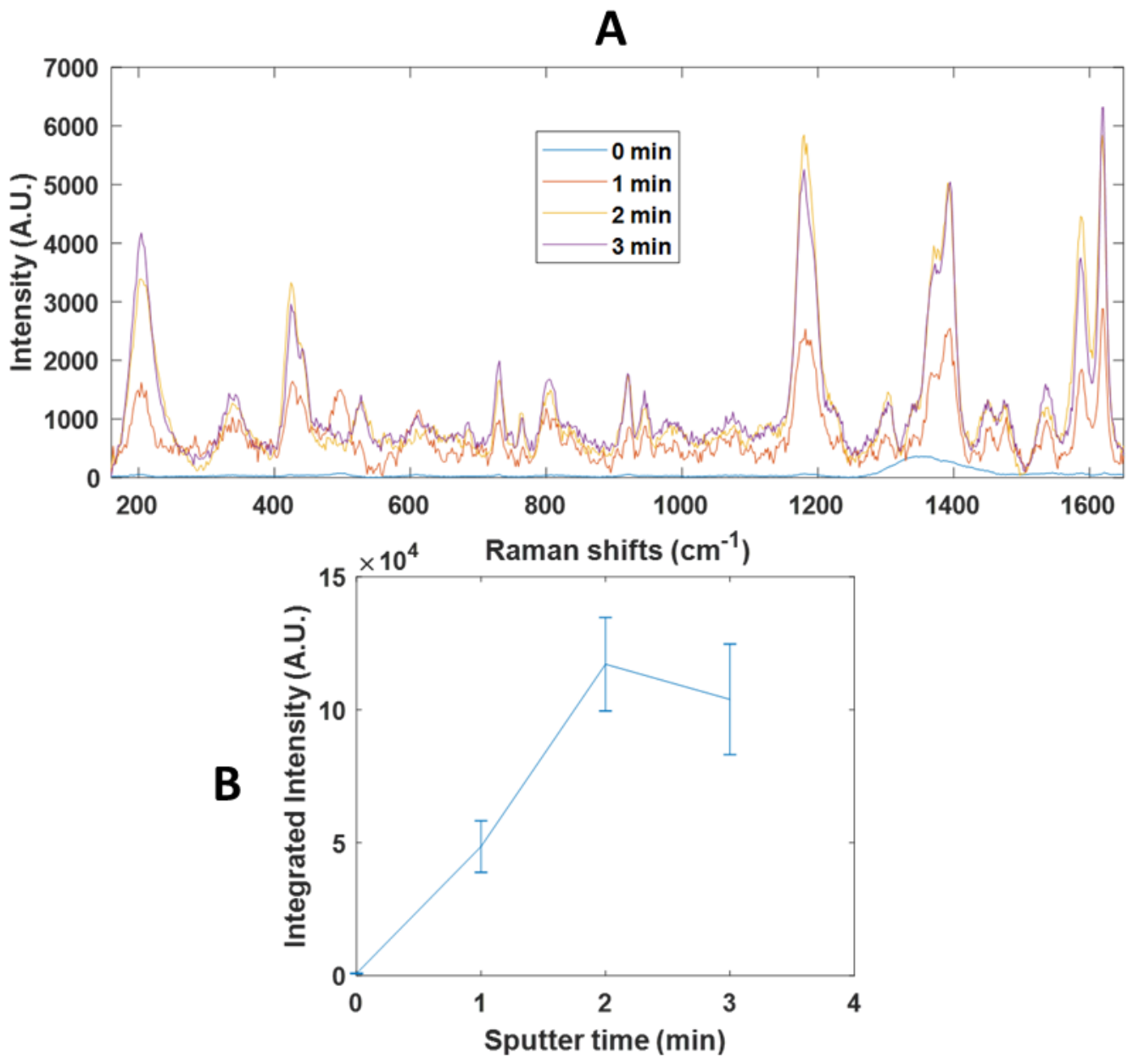

Figure 3-6 A) Background corrected SERS spectra for 1 min, 2min and 3min sputter coats. The blue bump located at $1300 \mathrm{~cm}^{-1}-1400 \mathrm{~cm}^{-1}$ region for $0 \mathrm{~min}$ is artificial due to the fluorescence background of the glass and the background suppression algorithm. B) Comparison of integrated intensity for the $1177 \mathrm{~cm}^{-1}$ peak for different sputter times. This peak was chosen due to its prominence and isolation from the surrounding peaks.

\section{Dependence of SERS activity on concentration of CV dye}

Different concentrations of CV dyes were tested to evaluate the concentration dependence of SERS as shown in Table 3-1 and each concentration was assigned a label. The SERS spectra obtained were processed to suppress the background and are displayed in Figure 3-7. The peak located at $1177 \mathrm{~cm}^{-1}$ was used to compare the SERS intensities for different concentrations. The broad peak located in the region of $1275 \mathrm{~cm}^{-1}-1550 \mathrm{~cm}^{-1}$ was due to the fluorescence background generated from the glass substrates. 
Table 3-1 Concentration tested for NSL SERS substrates

\begin{tabular}{c|c|c} 
Substrates & Concentration $(\mathrm{PPM})$ & Concentration $(\mu \mathrm{M})$ \\
\hline NSL-100 & 100 & 245 \\
NSL-10 & 10 & 24.5 \\
NSL-1 & 1 & 2.45 \\
NSL-0.1 & 0.1 & 0.245
\end{tabular}

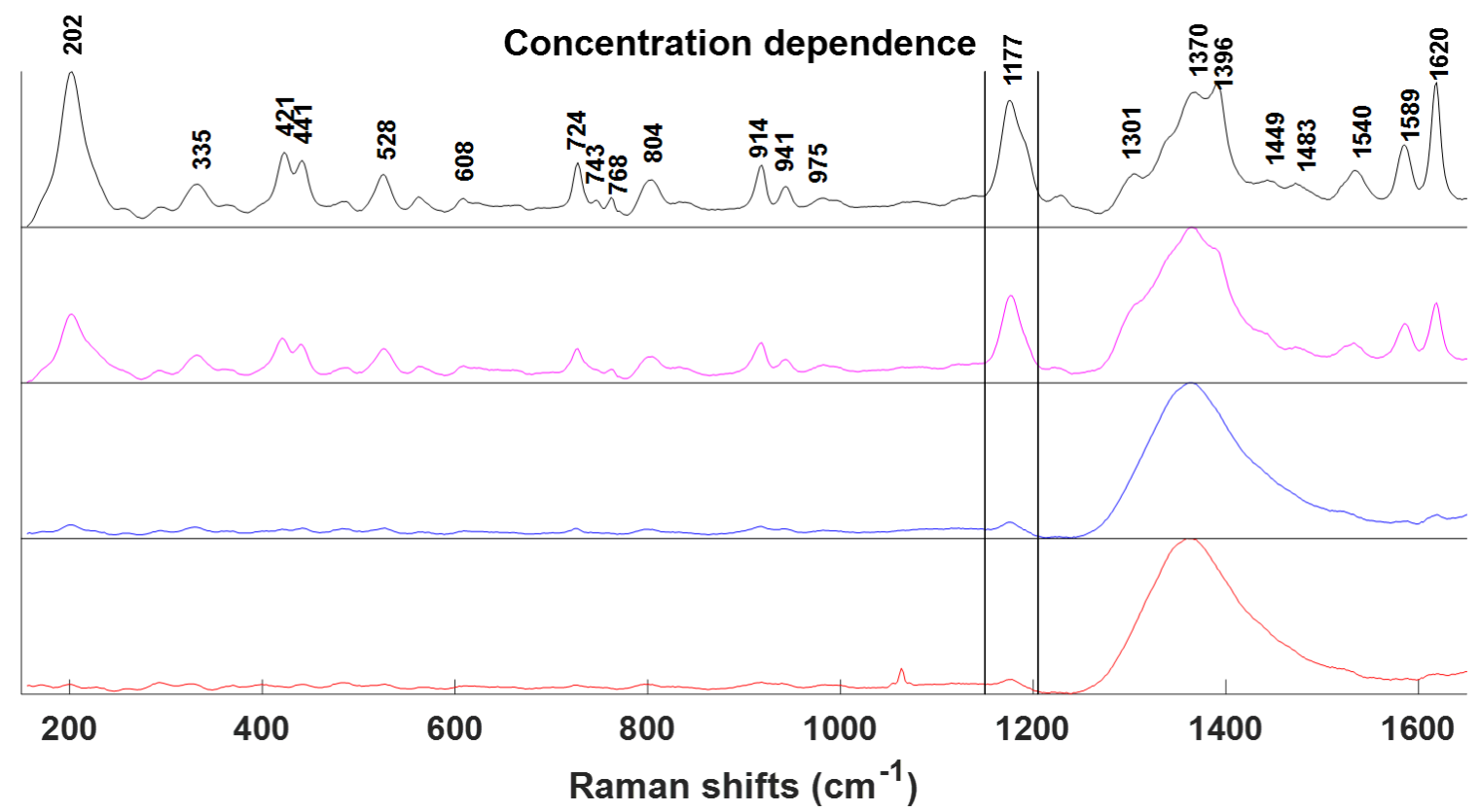

Figure 3-7 SERS spectra for NSL substrates plotted in the descending order of concentration from top to bottom (From NSL-100 - NSL-0.1). The vertical lines represent the peak examined to compute the AUC for comparing the intensities. The larger broad peak in the region of $1250 \mathrm{~cm}^{-1}$ to $1550 \mathrm{~cm}^{-1}$ is due to the fluorescence of the glass substrate.

The trend of SERS intensity for different concentrations is displayed in Figure 3-8. The errorbars are the standard deviation of SERS intensities. The standard deviation was computed for spectra obtained from a $10 \times 10$ scan raster scan in a $1 \mathrm{~mm} \times 1 \mathrm{~mm}$ region. For inter batch, the standard deviation was computed for scans of 3 different batches. Intra batch variations were found to be lower than the inter-batch variations. 


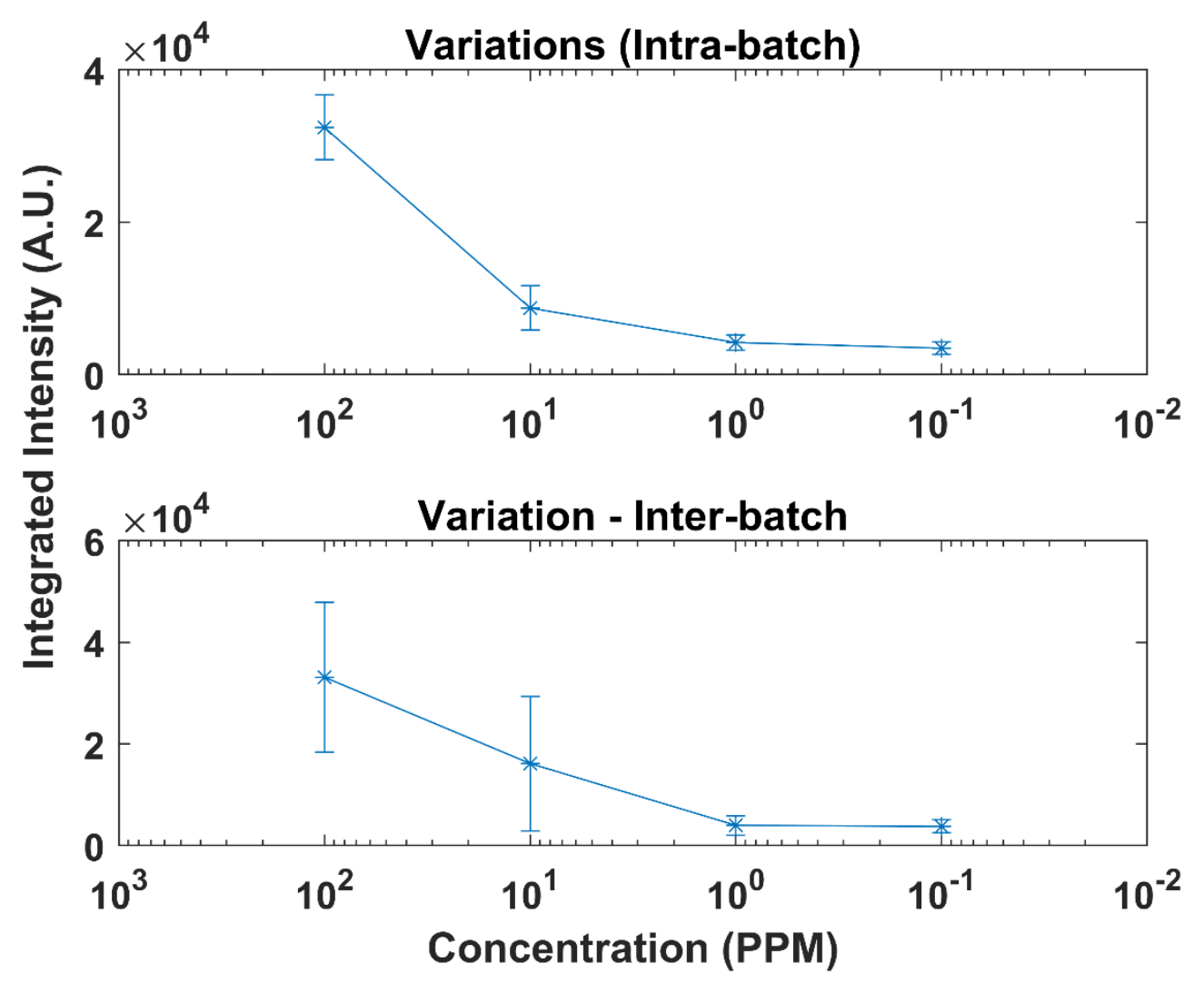

Figure 3-8 Concentration dependence of NSL SERS substrates and variation in fabricated batches.

Figure 3-9 depicts a 3D plot showing intra batch variation of the intensity of SERS spectra for NSL-100. The standard deviation for this case was found to be $10 \%$ which indicated uniformity over a large area $(1 \mathrm{~mm} \times 1 \mathrm{~mm})$. The transfer of monolayer from the air-water interface onto the surface of the glass was achieved. The inter-batch variability was found to be $40 \%$ for the NSL100. This was likely due to the compounding of the variations due to the NSL fabrication process and the variability in the sputtering process. 


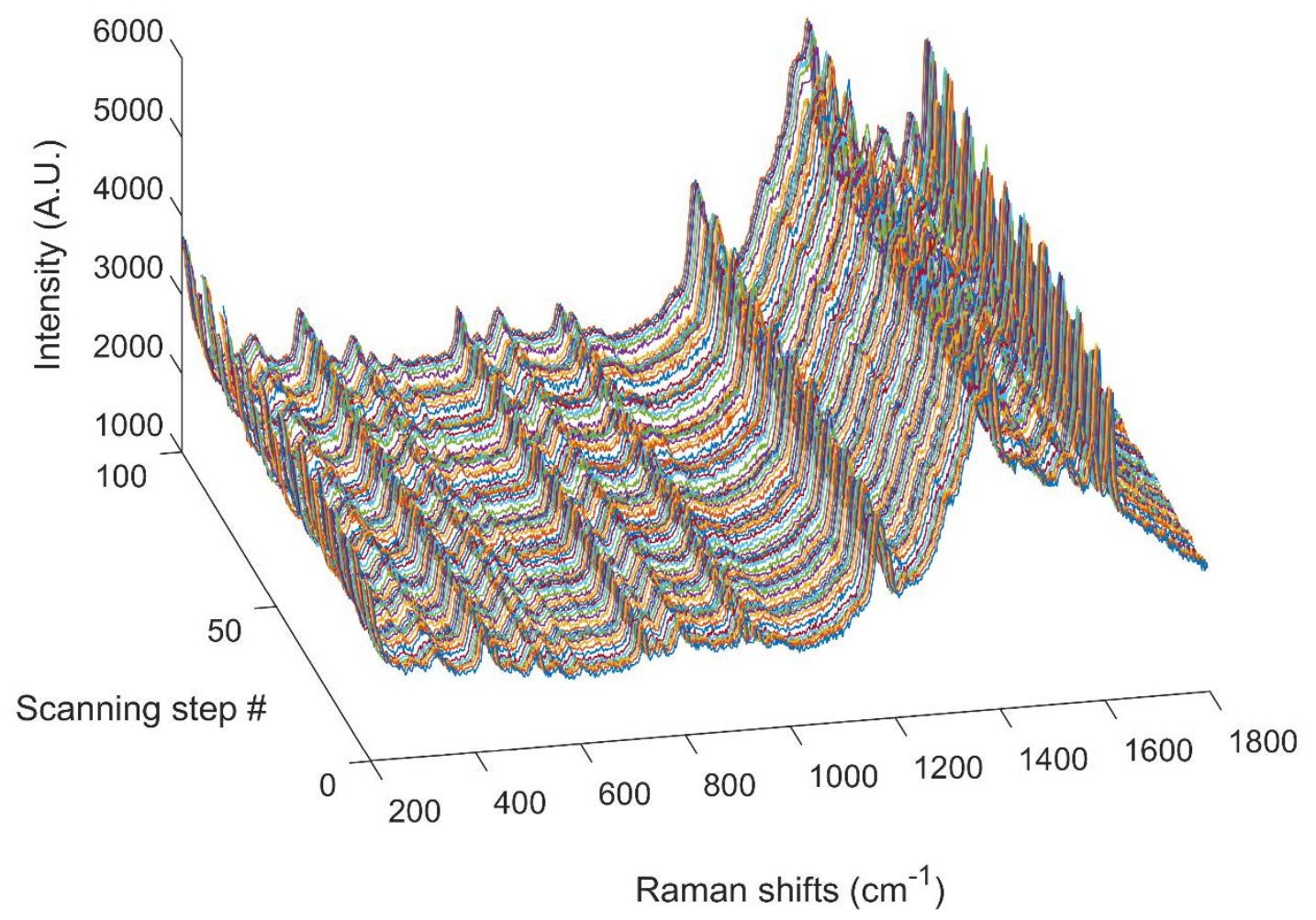

Figure 3-9 Intra batch spectra for NSL - 100 substrates.

Absorption spectra for the flat NSL substrates are shown in Figure 3-10. The mean value for the absorption peak was found to be $622 \mathrm{~nm}$ indicating the resonance wavelength of the nanoparticles formed on the substrate. The position of the absorbance peak of the samples was in general agreement (within 5\% maximum variation from the mean) with exception of an outlier at $564 \mathrm{~nm}$. 


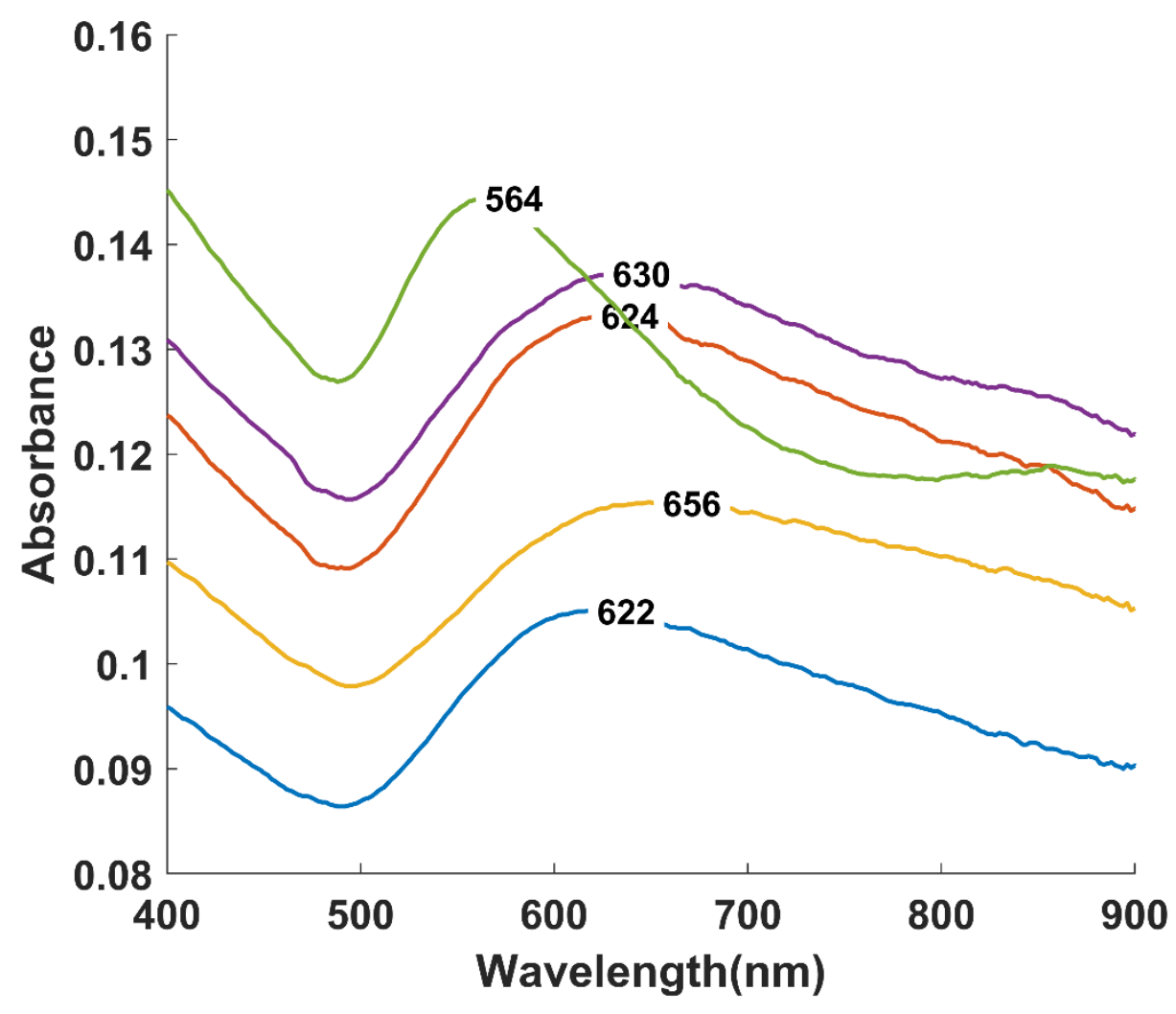

Figure 3-10 Absorption spectra of identically fabricated flat NSL substrates. Spectra from 5 identical substrates are presented.

\subsubsection{Fiber NSL substrates}

Optical fiber tips were prepared as described in the methods section. The optical fiber tips after they were scored with a scribing tool were mechanically pulled to break them at the score location as shown in Figure 3-11. The monolayer formed at the air-water interface was transferred onto the optical fiber tips. The images of fiber optic tips, presented in Figure 3-11, were processed to evaluate the coated surface area of the tips revealing voids that represented regions without the coverage of PS spheres. The optical fiber with the best coverage area of the coating was chosen as a reference fiber and area ratios were computed for each optical fiber and these ratios were used to normalize the SERS intensity. 


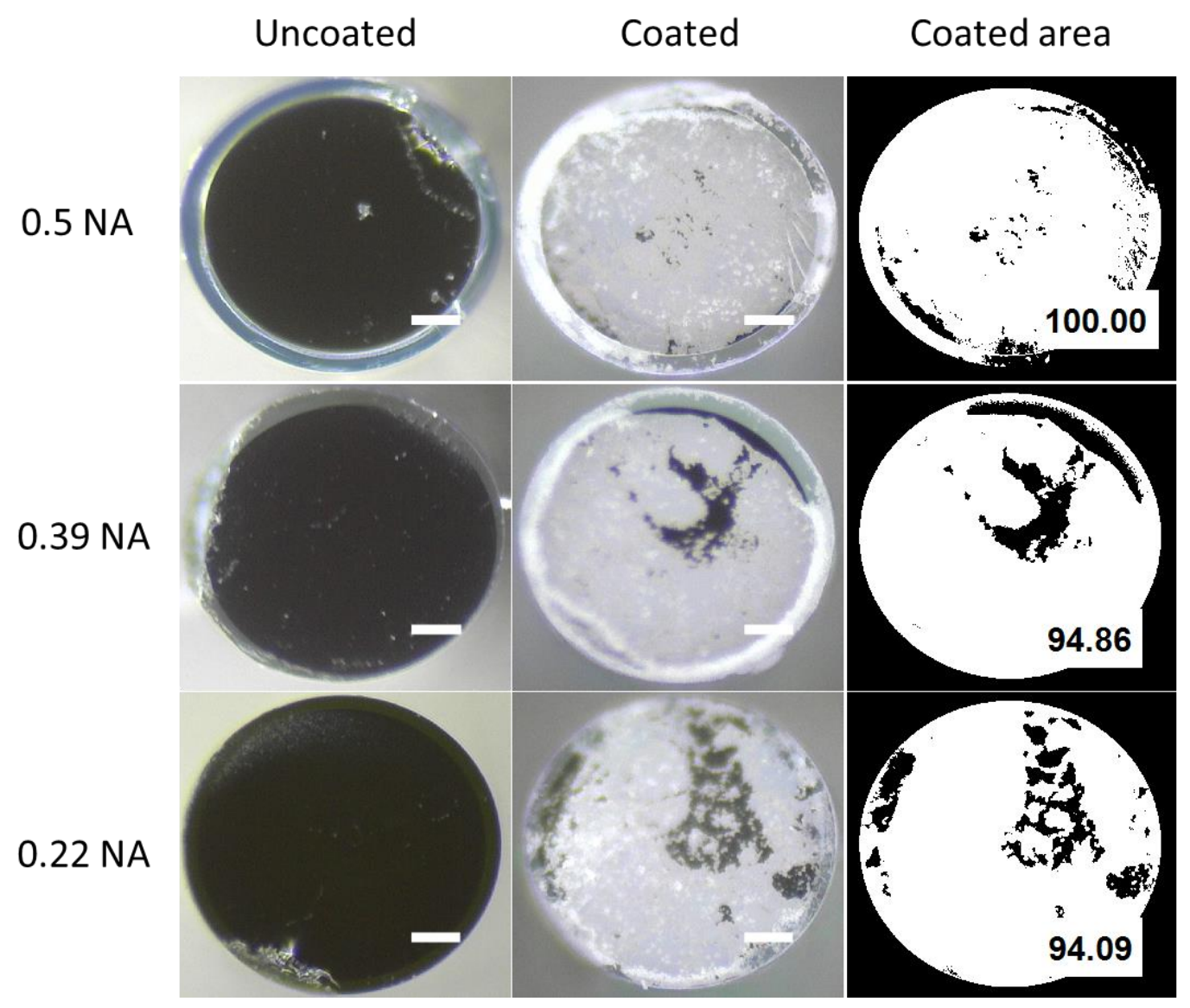

Figure 3-11 NSL fabrication process for optical fiber tips. Brightfield microscopic images of the prepared optical fiber tips are presented in the first column. The second column depicts tips coated with a monolayer of PS microspheres. The third column represents a binary image with thresholds applied to evaluate the coating area of the optical fibers.

The optical images show large area coverage of the fiber tips and corresponding SEM image revealed the monolayer formation as shown in Figure 3-12. The AFM image shows a hexagonal periodic array of curved triangular Au nanoparticles created after removal of the polystyrene mask. Line defects (line-like discontinuities in the tightly packed hexagonal lattice structure) were observed in the SEM images which are characteristic in NSL. 

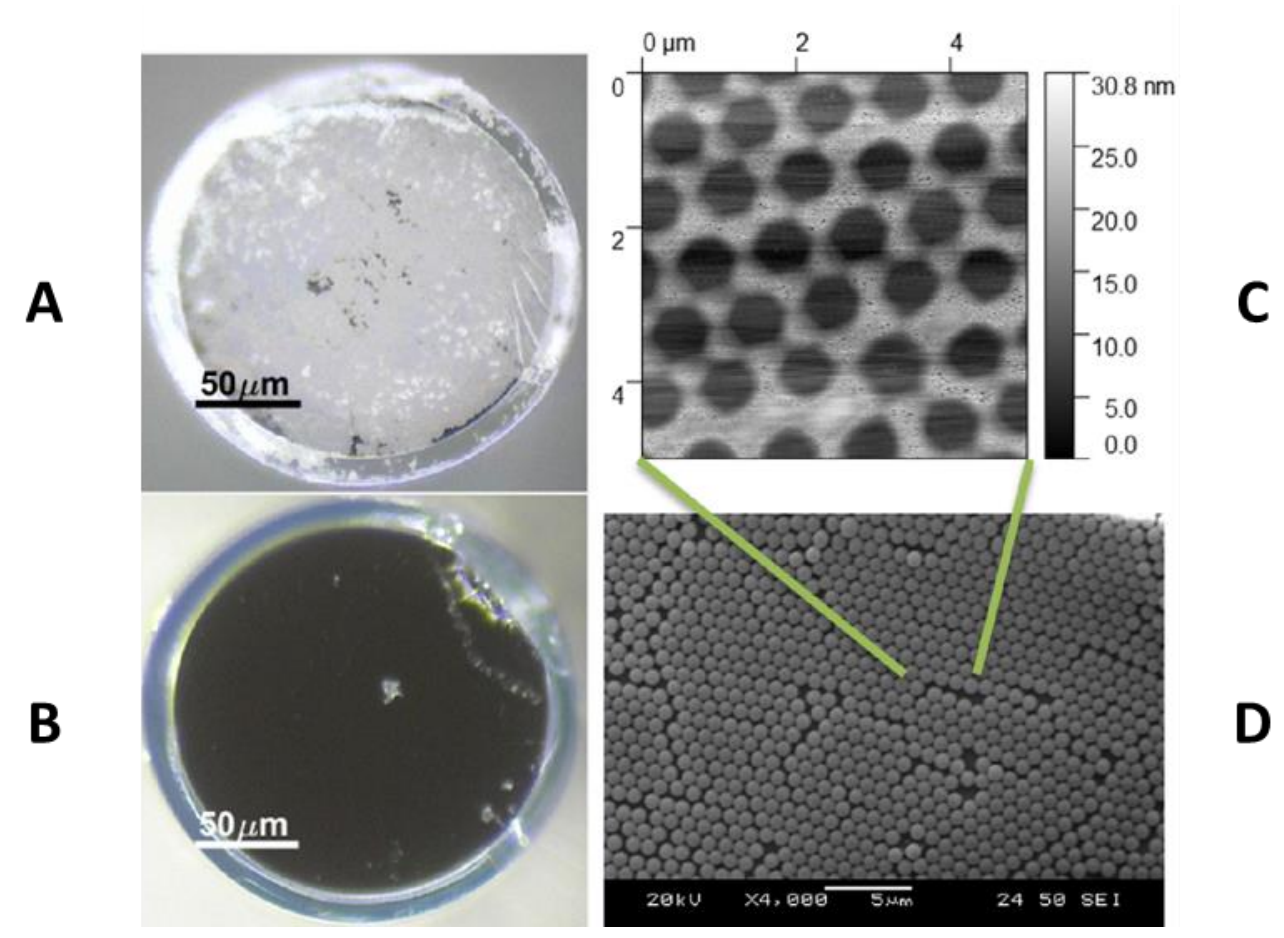

Figure 3-12 A) Fiber-optic tips after the NSL fabrication process B) AFM image showing the resultant nanopattern on the fiber tip C) Fibe-optic tip before NSL fabrication D) SEM image of the monolayer of PS spheres on the fiberoptic tip.

Figure 3-13 shows the background signals from optical fibers without nanostructured tips. The background from $0.22 \mathrm{NA}$ fiber was consistently distinct from the other fibers due to some interference from the fiber coating which was made of acrylate. In general, the region greater than $1300 \mathrm{~cm}^{-1}$ had a low background generated by the optical fiber. The fluorescence intensity was highest for the $0.5 \mathrm{NA}$ fiber below $700 \mathrm{~cm}^{-1}$. The trend was opposite for higher shifts which can be attributed to the variation in material properties of the optical fibers. The laser power at the focal spot was $16 \mathrm{~mW}$ and the power output through the fiber was $3.6 \mathrm{~mW}, 8.2 \mathrm{~mW}$ and $12.4 \mathrm{~mW}$ for the $0.2 \mathrm{NA}, 0.39 \mathrm{NA}$ and $0.5 \mathrm{NA}$ fibers respectively. 


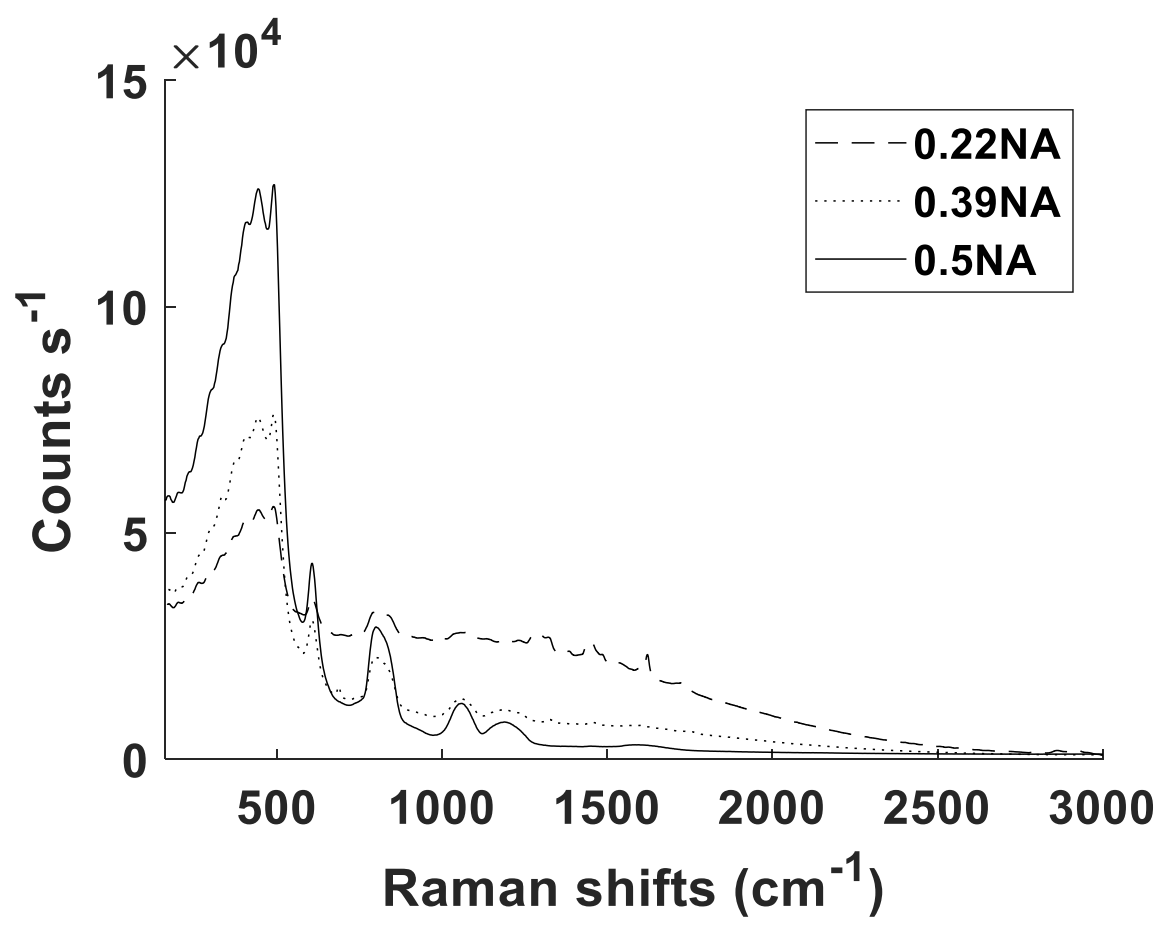

Figure 3-13 The autofluorescence background generated by the optical fibers used in the study.

Following the fabrication of SERS fibers, their performance was evaluated by using Crystal Violet (CV) as an analyte. SERS spectra were collected in the direct (laser incident on the nanofabricated face directly) and remote configurations (laser incident on the remote face opposite to the nanofabricated face). Figure 3-14 depicts the spectra obtained through direct and remote configurations. In the direct configurations, the acquired spectra for all fiber types were within $15 \%$ intensity variation (from the maximum value) confirming the similarity of the nanostructured tips for all three cases when illuminated directly on the fabricated tips. All the spectra obtained were normalized by the integration time and the laser power coupled through the optical fibers for different NA values. All spectra were corrected for background for visualization purposes. The optical fibers exhibit a strong background below $1100 \mathrm{~cm}^{-1}$. This decreases the quality of Raman spectra below this range. Figure 3-15 compares integrated intensity calculated using the tangent skim approach [135] of the peak centered at $1620 \mathrm{~cm}^{-1}$ in the region depicted by the dashed lines in Figure 3-14. This was chosen to ensure minimal interference from the fluorescence background signals generated by the optical fiber as it is in a region free from background fluorescence. As the collection efficiency of a fiber scales with the square of the NA, fit to NA squared is presented that 
depicts the trend of integrated Raman intensity. The integrated peak intensity variation shows good agreement with this fit.

For practical performance evaluation, Intensity Enhancement Factors (direct $e_{I}^{\text {fiber }}$ ) and remote $e_{I}^{\text {fiber }}$ were calculated using the following equations

$$
\operatorname{direct}_{I} e_{I}^{\text {fiber }}=\frac{\text { direct }_{\text {SERS }}^{\text {fiber }}}{\mathrm{I}_{\mathrm{RS}}^{\text {fiber }}}, \text { remote }_{I}^{\text {fiber }}=\frac{\text { remote } \mathrm{I}_{\mathrm{SERS}}^{\text {fiber }}}{\mathrm{I}_{\mathrm{RS}}^{\text {fiber }}}
$$

$\operatorname{direct} e_{I}^{\text {fiber }}$ was calculated as 31 and 37 for 0.39 NA and 0.5 NA optical fibers respectively. remote $e_{I}^{\text {fiber }}$ was found to be 11 and 36 for 0.39 NA and 0.5 NA optical fibers respectively. This showed us that $0.5 \mathrm{NA}$ fiber performed similar in remote and direct configurations and the performance of 0.39 NA degraded in the remote configuration when compared to 0.5 NA. The $e_{\mathrm{I}}^{\text {flat }}$ was found to be 30 indicating that the 0.5 NA fiber performed slightly better which can be attributed to the larger area of collection of the optical fiber (which had $200 \mu \mathrm{m}$ diameter compared to $30 \mu \mathrm{m}$ spot size of the RS collection setup). These intensity enhancement factors correspond to total enhancement factors in the order of $10^{4}-10^{5}$ after including the molecular contribution ratios (which can be estimated to be in the range of $10^{3}$ [127]). This value is in accordance to the value obtained by similar studies [107]. The major difference in this work was the use of a custom setup that can ensure that the full collection area of the optical fiber was utilized for data collection. Using a standard Raman microscope setup for such studies would result in collection of only a portion of light back-propagating in the fiber. 

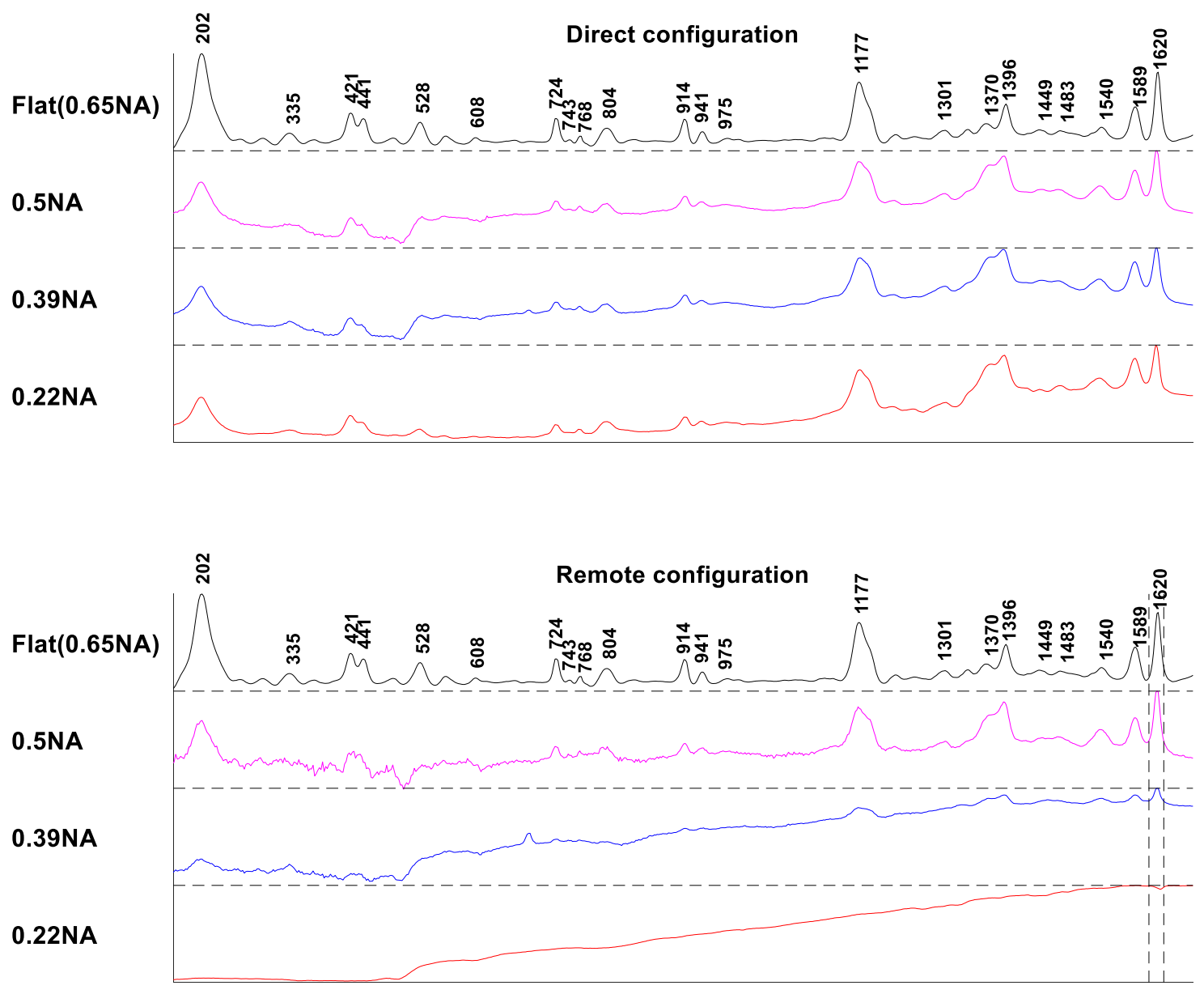

Raman shifts $\left(\mathrm{cm}^{-1}\right)$

Figure 3-14 The spectra are arranged in decreasing order of NA from top to bottom and are normalized for visualization purposes. The spectra are offset equally, and the topmost spectrum was acquired using a flat substrate fabricated on a microscopic cover slip. The vertical dashed lines in the remote configuration mark the peak (1620 $\mathrm{cm}^{-1}$ ) that was used to compare the peak intensity for different NA. The spectra are normalized (the scales of each subplot separated by dashed horizontal lines is between 0 and 1) for better depiction of the peaks. Although the spectra were corrected for background, some of the resultant artefacts related to the strong background were still present in the processed spectra. 


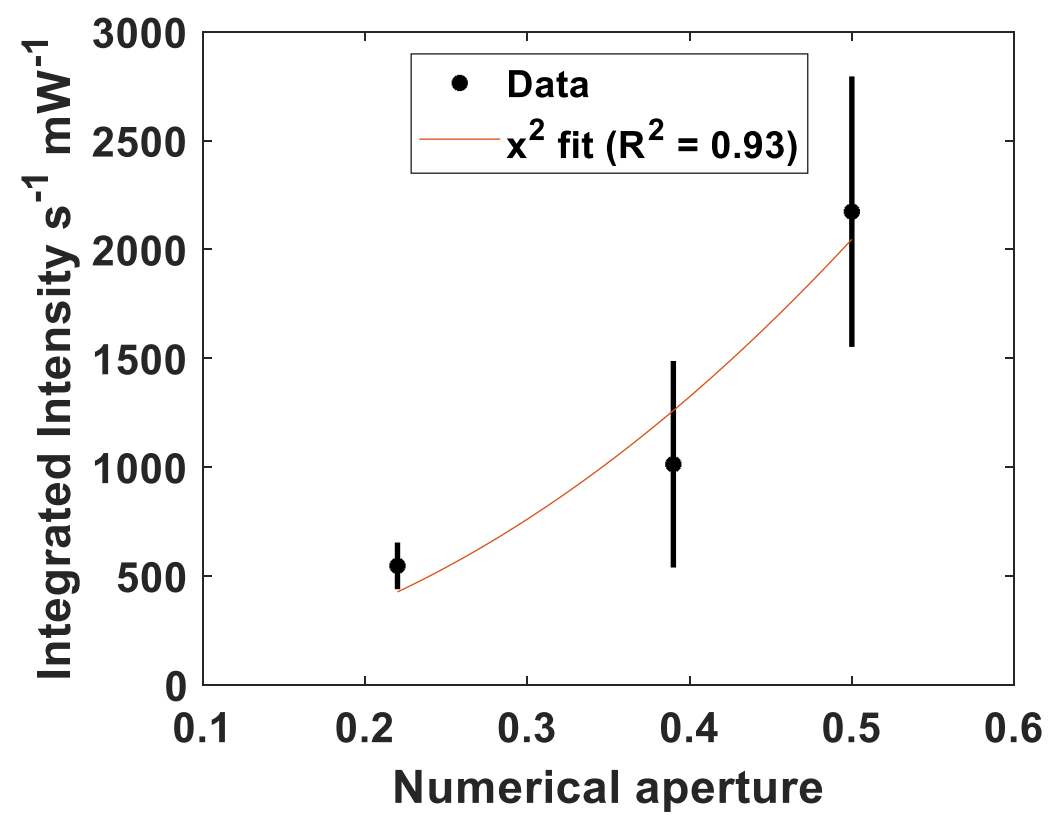

Figure 3-15 Integrated peak corresponding to the dashed lines in Figure 3-14 with a fit to NA ${ }^{2}$. The vertical lines for each point represent one standard deviation.

\subsection{Nanoporous substrates}

Figure 3-16 shows topographical AFM images of the NS substrates. The image obtained for the NS-7 substrates was of lower quality due to the smaller size of the pores. Also, due to the smaller sizes of the pores, the true depths of the pores could not be assessed as the AFM tip could not penetrate the smaller pores. The pores did not have any specific orientation and the depths obtained with AFM do not truly represent the pore depths for both the substrates. The sizes of the pores were obtained from the manufacturers [93]. The pore size estimated using the methods were used to label the substrates as NS-7 (for $7 \mathrm{~nm}$ pores) and NS-17 (for $17 \mathrm{~nm}$ pores). 


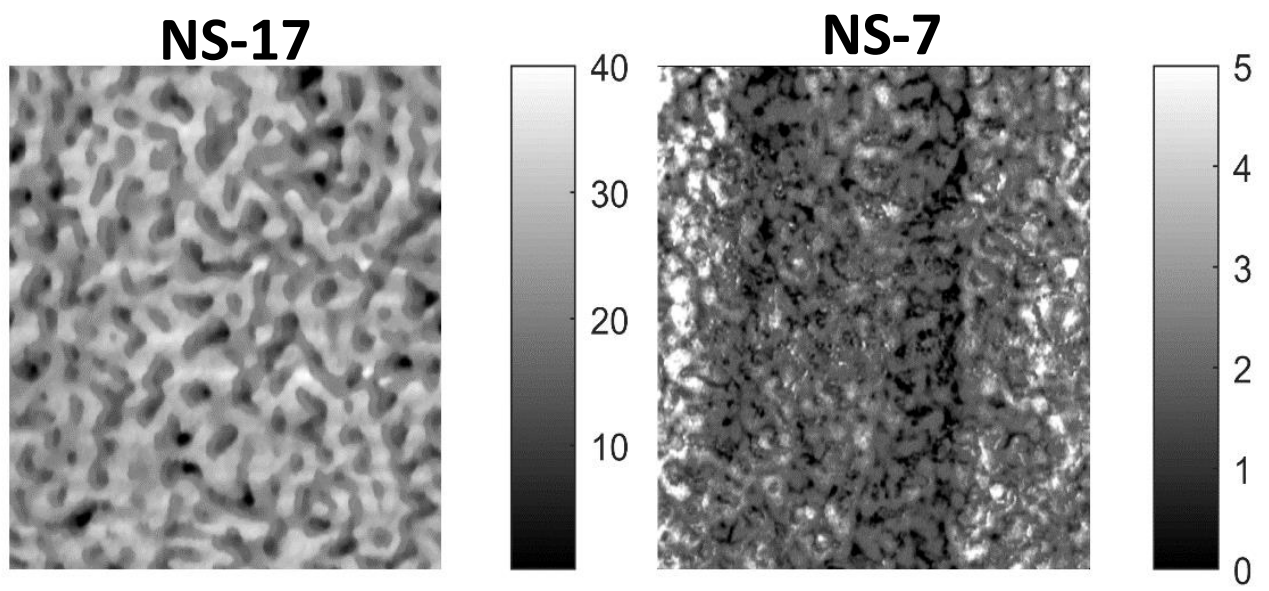

Figure 3-16 AFM images of NS-17 and NS-7 substrates, the AFM scan area was $800 \mathrm{~nm} \times 800 \mathrm{~nm}$.

The sizes of pores were also evaluated from the AFM images. The image profiles were extracted in a spoke wheel pattern as described in the methods section. The pore size distribution for the NS7 and NS-17 substrates are shown in Figure 3-17. For NS-7 substrates, the distribution showed a peak at around $7 \mathrm{~nm}$ which was in good agreement with the stated pore sizes. For NS-17 substrates, the distribution of pore sizes had a broad peak ranging from $20 \mathrm{~nm}$ to $30 \mathrm{~nm}$. The pore sizes acquired were larger because at the surface, the pore opening was found to be larger and the pore likely narrowed inside the nanoporous structure. 


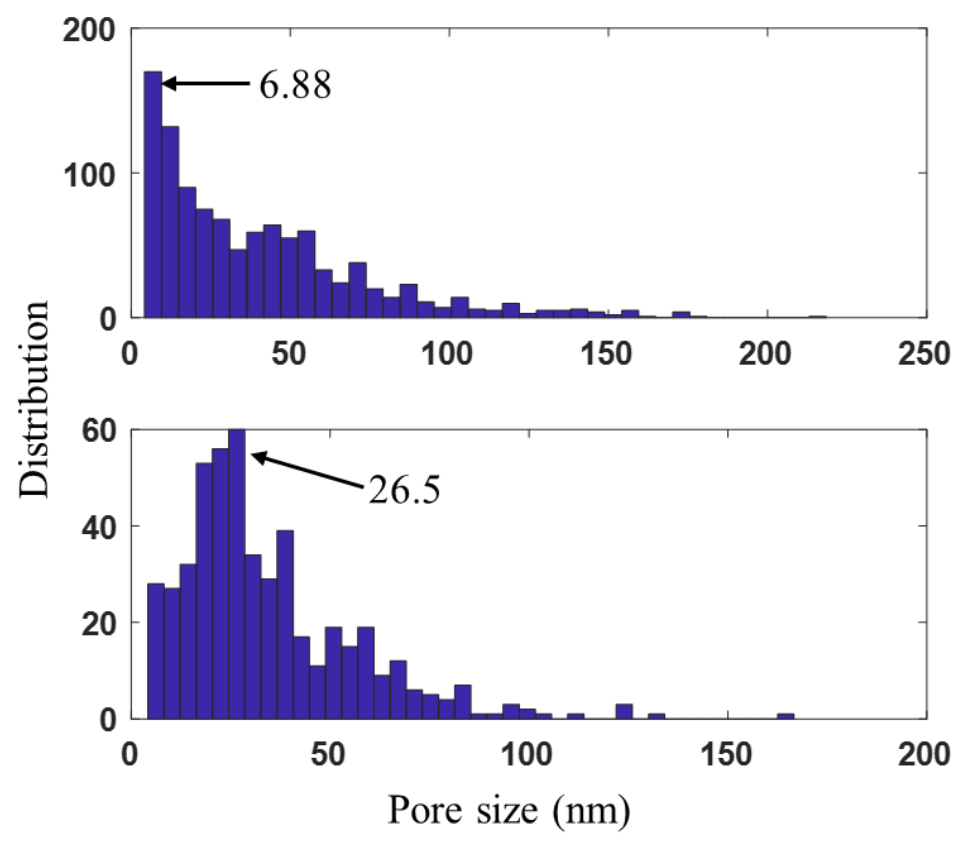

Figure 3-17 Pore size distribution for NS-7 and NS-17 substrates.

Figure 3-18 shows topographical AFM images of the Au coated NS substrates. For the NS-7, the bare nanoporous structure was visible up to the $25 \mathrm{~nm}$ Au layer thickness whereas the structure was lost for the $50 \mathrm{~nm} \mathrm{Au} \mathrm{layer.} \mathrm{The} 12 \mathrm{~nm}$ and $25 \mathrm{~nm}$ thickness coatings were visibly like the uncoated NS substrates and the $75 \mathrm{~nm}$ layer was visibly like the $50 \mathrm{~nm}$ layer. The images presented depict a trend that increasing thickness of gold coating led to pore filling which occurred rapidly for the NS-7 substrates in comparison to the NS-17 substrates due to the smaller pore sizes of the NS-7 substrates. 


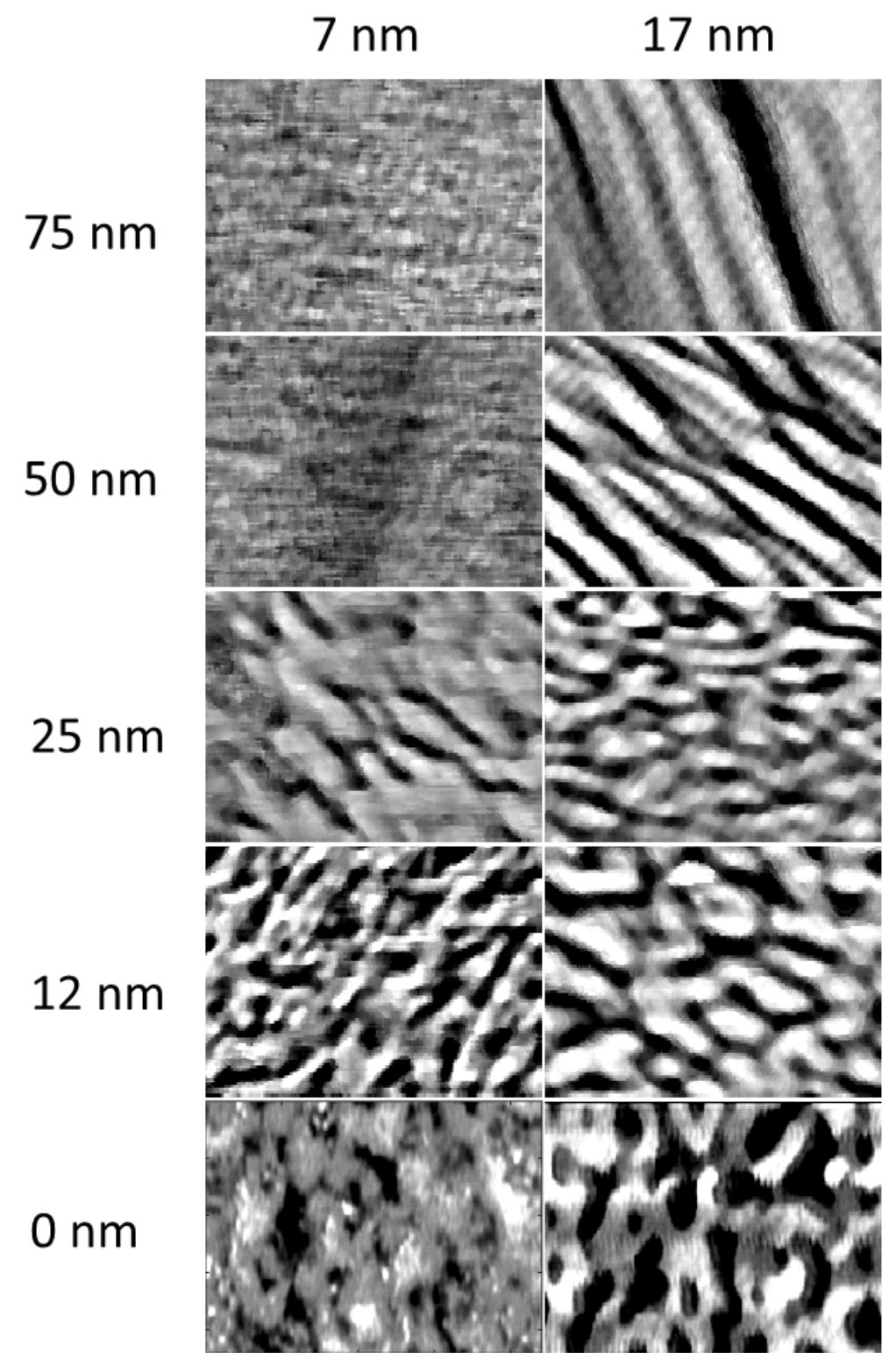

Figure 3-18 AFM images depicting the surface of NS-7 and NS-17 substrates after sputter coating of Au layers.

Figure 3-19 depicts the pore size distribution for Au coated NS-7 and NS-17 substrates. The pore sizes were computed like the uncoated NS substrates. Distributions of pore sizes for all the substrates are presented in Figure 3-19. To observe the trend of changes in the pore size with Au layers, a ratio of pore counts with sizes less than $20 \mathrm{~nm}$ to the pore counts with sizes larger than $20 \mathrm{~nm}$ was computed and shown in Figure 3-20. 


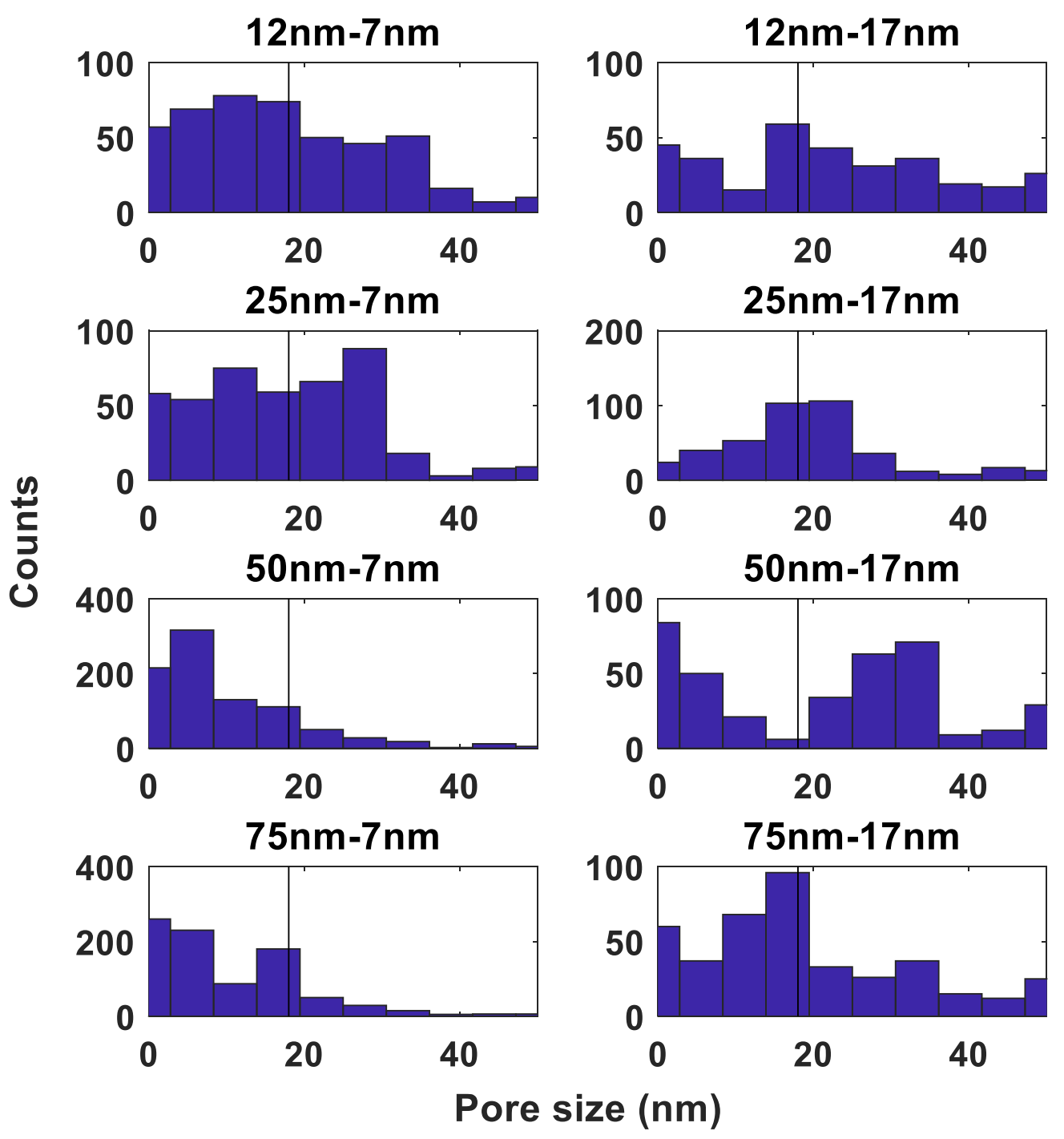

Figure 3-19 Pore size distribution for Au coated NS-7 and NS-17 substrates.

Figure 3-20 shows the ratio of pore counts with sizes less than $20 \mathrm{~nm}$ to the pore counts with sizes larger than $20 \mathrm{~nm}$ for different thickness of Au layers. The slope of the ratio was higher for NS-7 substrates depicting a rapid filling of pores when compared to the NS-17 substrates. 


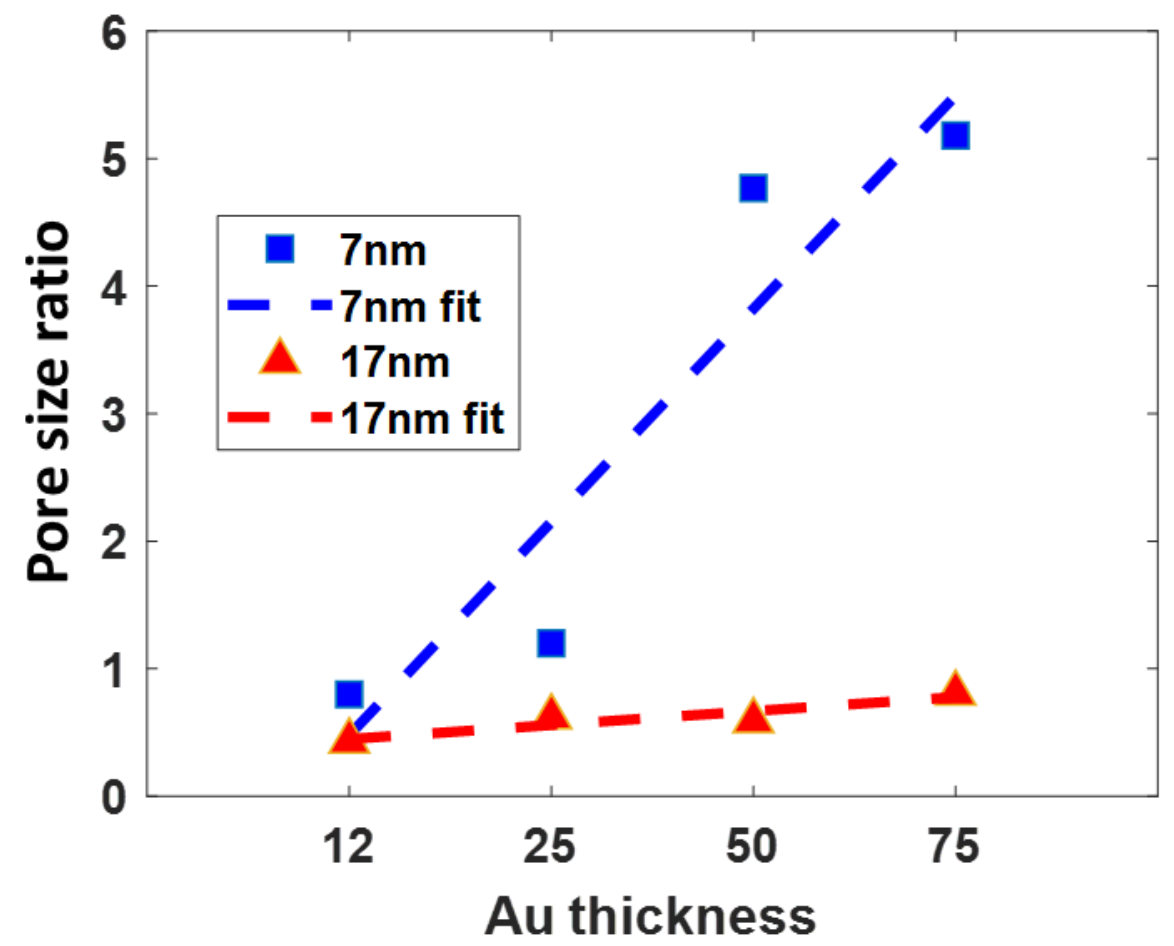

Figure 3-20 Variation of pore sizes with Au layer thickness for NS-7 and NS-17 substrates depending on coating thickness. The slope is larger for the NS-7 substrates indicating that the pores filled up faster.

SERS activity was compared for varying thickness of Au layers using 10 PPM CV dye. Figure 3-21 shows the SERS spectra of CV dye for NS-7 and NS-17 substrates for $12 \mathrm{~nm}, 25 \mathrm{~nm}, 50 \mathrm{~nm}$ and $75 \mathrm{~nm}$ of Au layer thickness. The spectra were normalized to maximum value and are offset for clarity. This was done for visualization purposes hence they do not display the decrease of intensity as the thickness of Au increased. For observing the thickness dependence, Figure 3-22 A plots the area under the curves for various thicknesses of Au. For the AuNS-7 substrates, the AUC values were highest for $25 \mathrm{~nm}$ thickness of Au while for the NS-17 substrates, the AUC value was highest for $50 \mathrm{~nm}$. AuNS-7 substrates exhibited larger overall enhancements indicating higher local field enhancements for smaller pore sizes. AuNS-7 substrates had the best enhancements for $25 \mathrm{~nm}$ Au thickness. For AuNS-17 substrates, there were no notable differences in enhancements up to $50 \mathrm{~nm}$ Au thickness after which the enhancements diminished. For AuNS-7, the maximum enhancements were 4 times higher when compared to the AuNS-17 (Figure 3-22 A). These findings suggest that the nanoroughness plays a major role in the SERS activity and smaller pore sizes can achieve larger enhancements. Intensity enhancement factors calculated for 10PPM 
concentration and the maximum Intensity Enhancement Factors (IEF) for AuNS-7 and AuNS-17 were 164 and 45 respectively. Uncoated NS matrices incubated in CV were used as control substrates for IEF calculations.
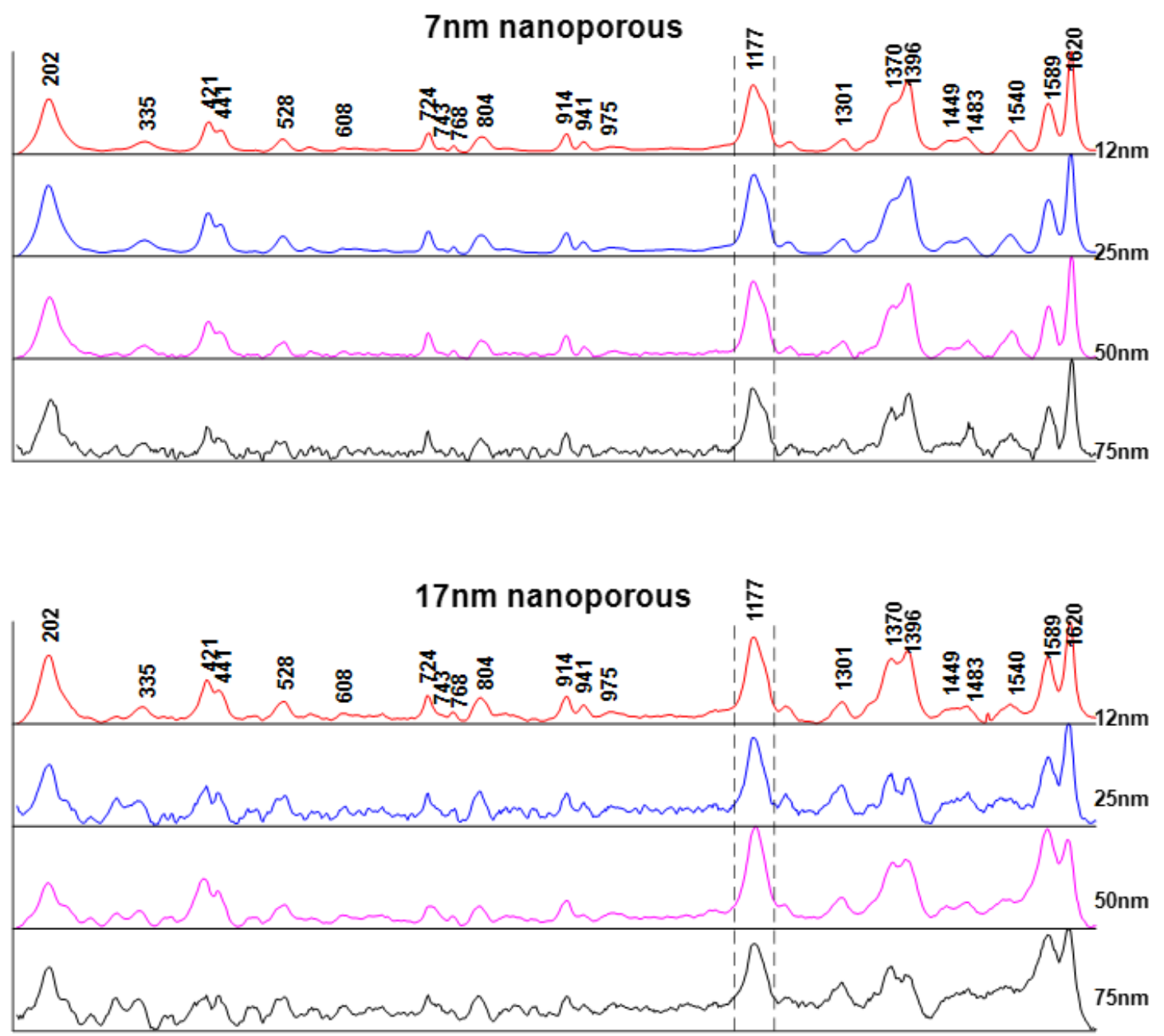

Figure 3-21 SERS spectra for NS-7 and NS-17 substrates with varying Au layer thicknesses.

To observe concentration dependence for the substrates, 0.1, 1 and 10 PPM of CV were used as analytes. Figure 3-22 B shows the AUC calculations for different concentrations of CV dye. AuNS-7 exhibited higher SERS activity compared to the AuNS-17. The characteristic CV peaks were not visible for 0.1PPM CV dye. Further concentrations are required to establish an exact limit of detection (LoD) which is expected to be less than 1PPM. It was shown that using a simple and inexpensive approach, a SERS active substrate was created using NS matrices. 
Optical fibers can also be prepared with borosilicate glasses which can be used to create nanoporous substrates. The technique described in the methods section for creating planar nanoporous substrates can be extended to be used with fibers to create a nanoporous sensing optical fiber.
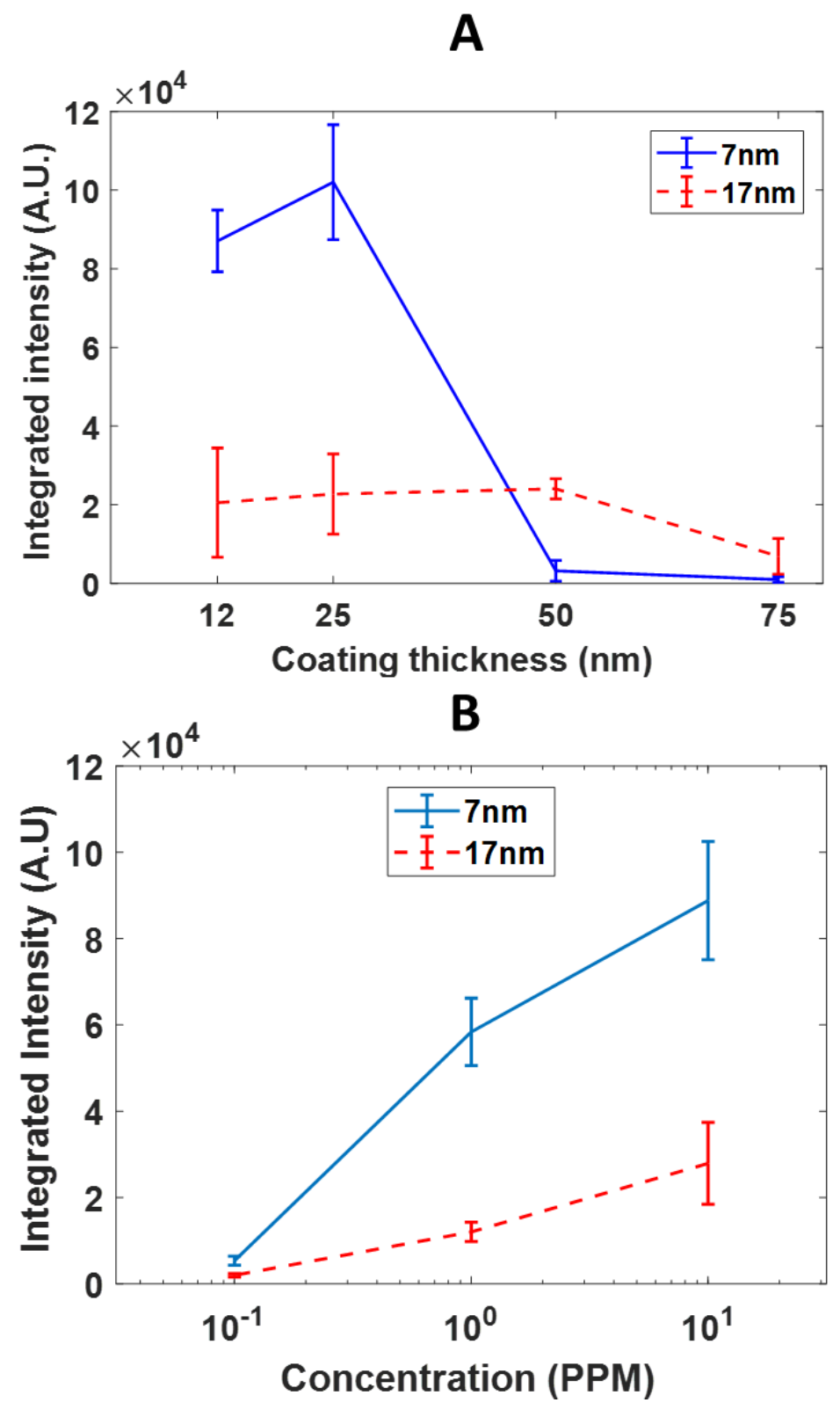

Figure 3-22 A) Dependence of SERS intensities on thickness of Au coating. B) Concentration dependence of SERS intensities. 


\subsection{Theoretical modeling}

\subsubsection{NSL - Single particle simulations}

The electric field of a single nanotriangle for different wavelengths is presented in Figure 3-23. The nanotriangles were surrounded by glass at the base and air at the top. The images show the $\mathrm{XY}$ plane $0.1 \mathrm{~nm}$ above the boundary of the glass and nanoparticle. This was done to avoid any boundary artefacts. Extinction cross section computed for the

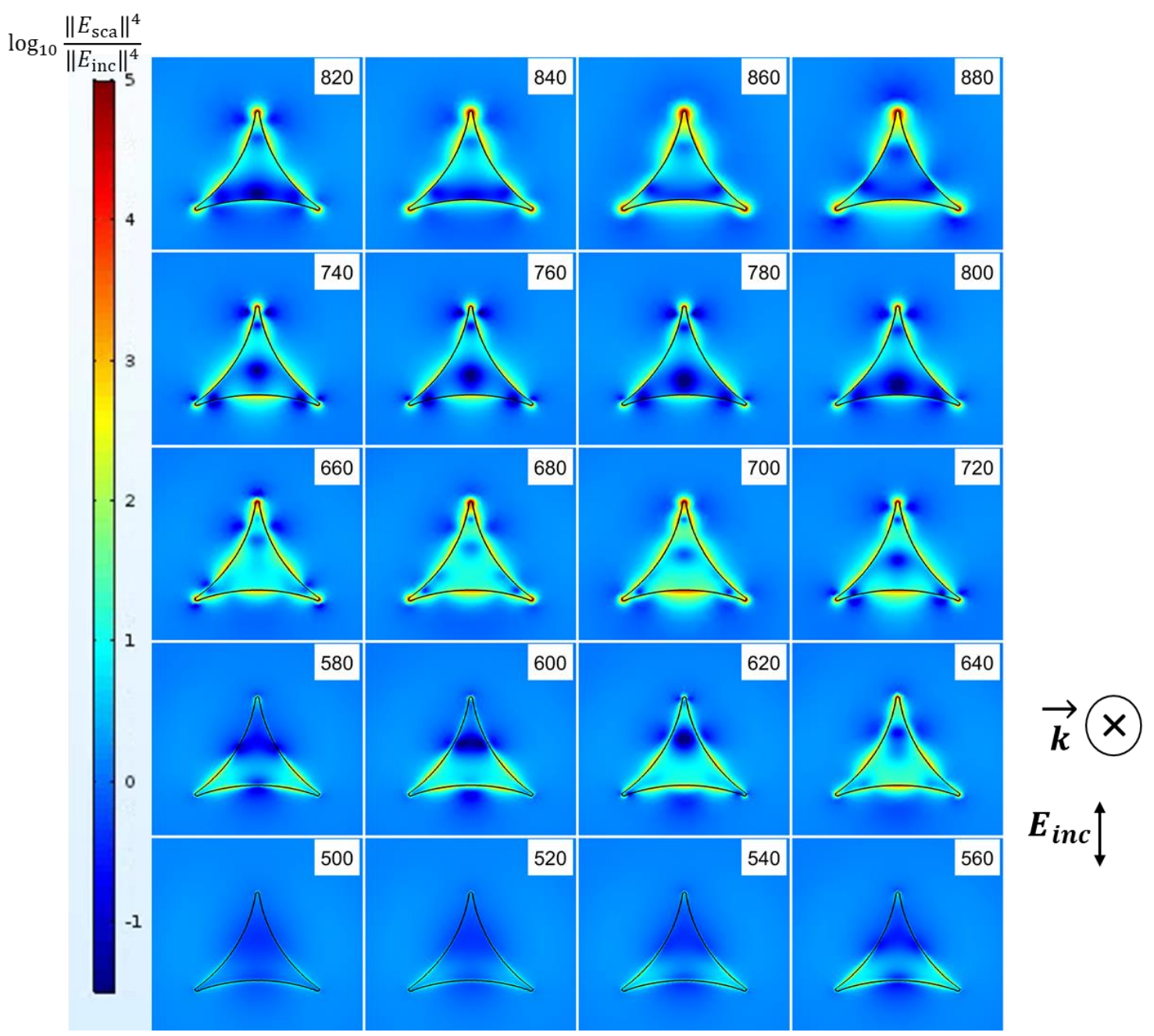

Figure 3-23 $\boldsymbol{e}_{n s l}^{c o m p}$ for single nanotriangles for the wavelength range $500 \mathrm{~nm}-880 \mathrm{~nm}$. The wave propagation direction and the polarization of the electric field is depicted on the figure.

nanoparticles is shown in Figure 3-24. The experimental data represents an average absorption spectrum obtained from 5 individually fabricated substrates. The simulations were found to match 
the resonance peak obtained experimentally within $5 \%$ of the experimental value. Two peaks were obtained from the simulations one at $660 \mathrm{~nm}$ and other at $840 \mathrm{~nm}$. The $840 \mathrm{~nm}$ peak was not observed experimentally which was likely due to polarization of the incident wave for simulations and the variability in the structure of the nanoparticles fabricated experimentally. Single particles were simulated whereas experimentally, an array of particles with interconnected edges were observed. The two peaks depicted in the spectra likely represent the quadrupole oscillations of the localized surface plasmon modes while the third peak (visible at $800 \mathrm{~nm}$ for $0.25 \mathrm{scaling}$ ) represents the dipole oscillations.

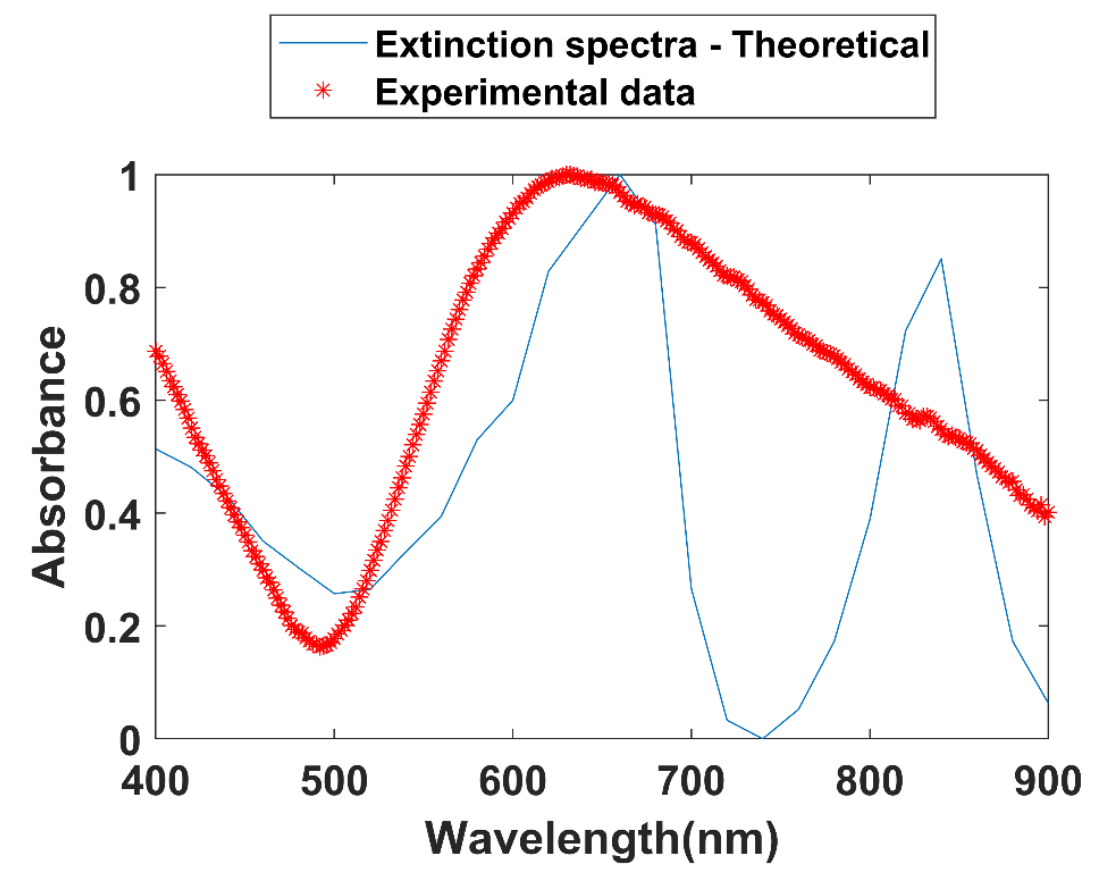

Figure 3-24 Extinction spectra obtained from simulations compared with experimental data.

\subsubsection{Effect of scaling the size of the nanoparticle}

Three scaling factors were utilized to scale the size of the nanoparticles isotopically to observe size dependent effects on the extinction spectrum and enhancement factors. The nanoparticle geometry was scaled down by a factor of 0.5 and 0.25 . The extinction spectra of the scaled nanoparticles are shown in Figure 3-25. The smallest scaling factor depicted a strong peak centered at $800 \mathrm{~nm}$. The peak at $800 \mathrm{~nm}$ for the scaling factor 0.25 represents the additional peak that is blue shifted as the size of the nanoparticle decreases as shown in Figure 3-25. 


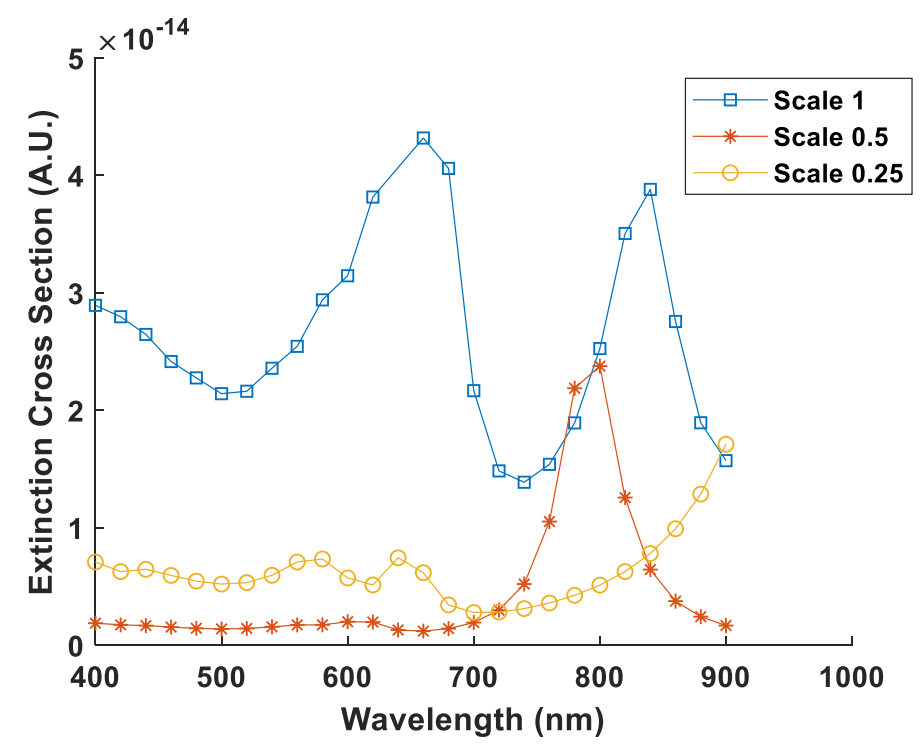

Figure 3-25 Dependence of extinction spectra on isotropic scaling of nanoparticle size.

The enhancement factors were found to be the largest for the smallest scaling of nanoparticle. This is likely because of the decreased radiation damping that agrees with other studies [136].

\subsubsection{Effect of height of the nanoparticle on extinction spectra}

The nanoparticle height was linearly varied from $15 \mathrm{~nm}$ to $45 \mathrm{~nm}$ with a step size of $7.5 \mathrm{~nm}$. The extinction spectra of the scaled nanoparticles are shown in Figure 3-26. Increasing the height (or thickness) of the nanoparticles resulted in blue shift of the resonance peaks for the nanoparticles. The results were found to agree with studies performed by other groups [78]. The extinction cross section also increased with height due to increased absorption and scattering by the larger nanosphere. Since for SERS $785 \mathrm{~nm}$ wavelength was used, smaller heights of nanoparticles were found to be more appropriate. 


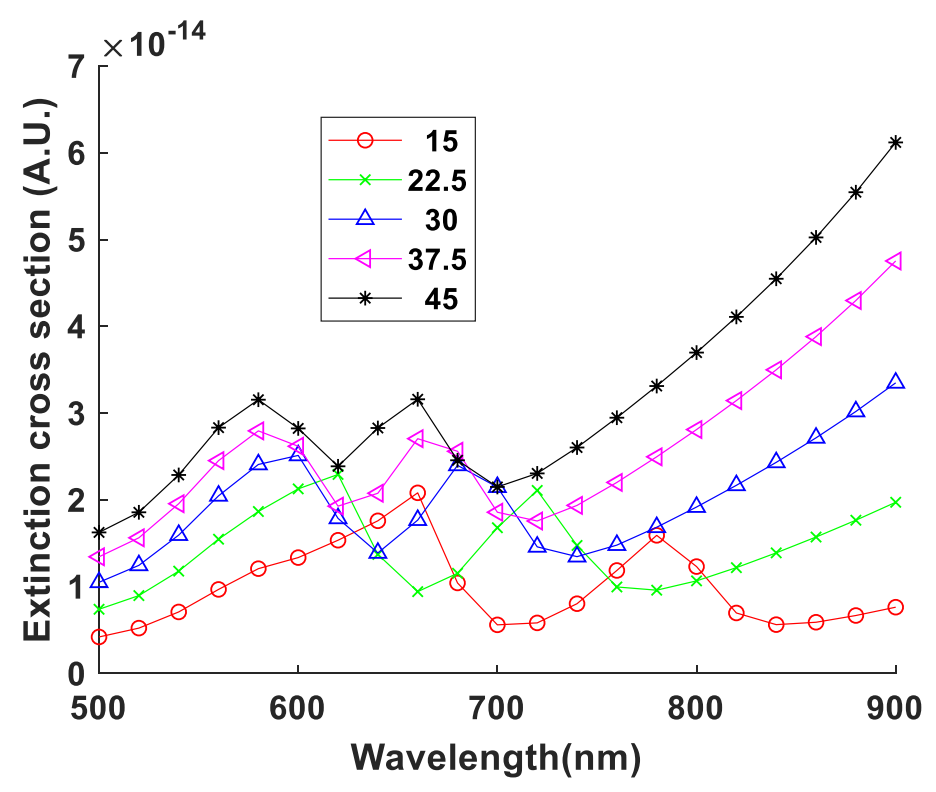

Figure 3-26 Dependence of extinction spectra on the height of the nanoparticle.

\subsubsection{Effect of height of the nanoparticle on enhancement factors}

The enhancement factors for each nanoparticle height are shown in Figure 3-27. Nanoparticles with $15 \mathrm{~nm}$ heights exhibited the largest enhancement factors. The peak enhancement factors followed the trend observed in shifts of extinctions spectra. The enhancement factors rapidly changed for nanoparticle heights of $15 \mathrm{~nm}$ and $22.5 \mathrm{~nm}$ after which the change was gradual. 


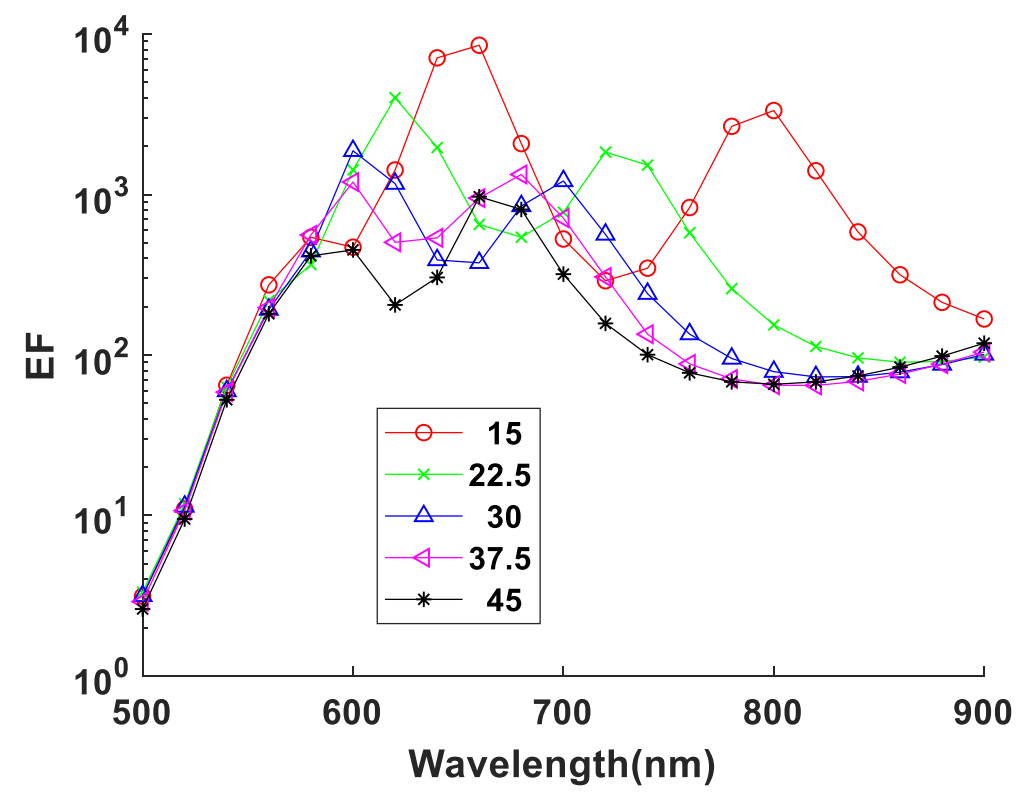

Figure 3-27 Dependence of enhancement factors on the height of the nanoparticle

\subsubsection{NSL - hexagonal array simulations}

NSL results in formation of curved nanotriangles arranged in a hexagonal periodic array. Floquet boundary conditions were applied to all the boundaries of the simulations to simulate an infinite periodic array. Figure 3-28 depicts the simulation results for wavelengths ranging from $500 \mathrm{~nm}$ $880 \mathrm{~nm}$. The general trend of enhancement was found to be like the single nanoparticle simulation. There were specific wavelengths where the periodic array simulation resulted in depiction of extra modes specific to the periodicity of the nanostructure. These were absent from the single particle simulation and show specific inter-particle coupling which results in larger enhancements. 


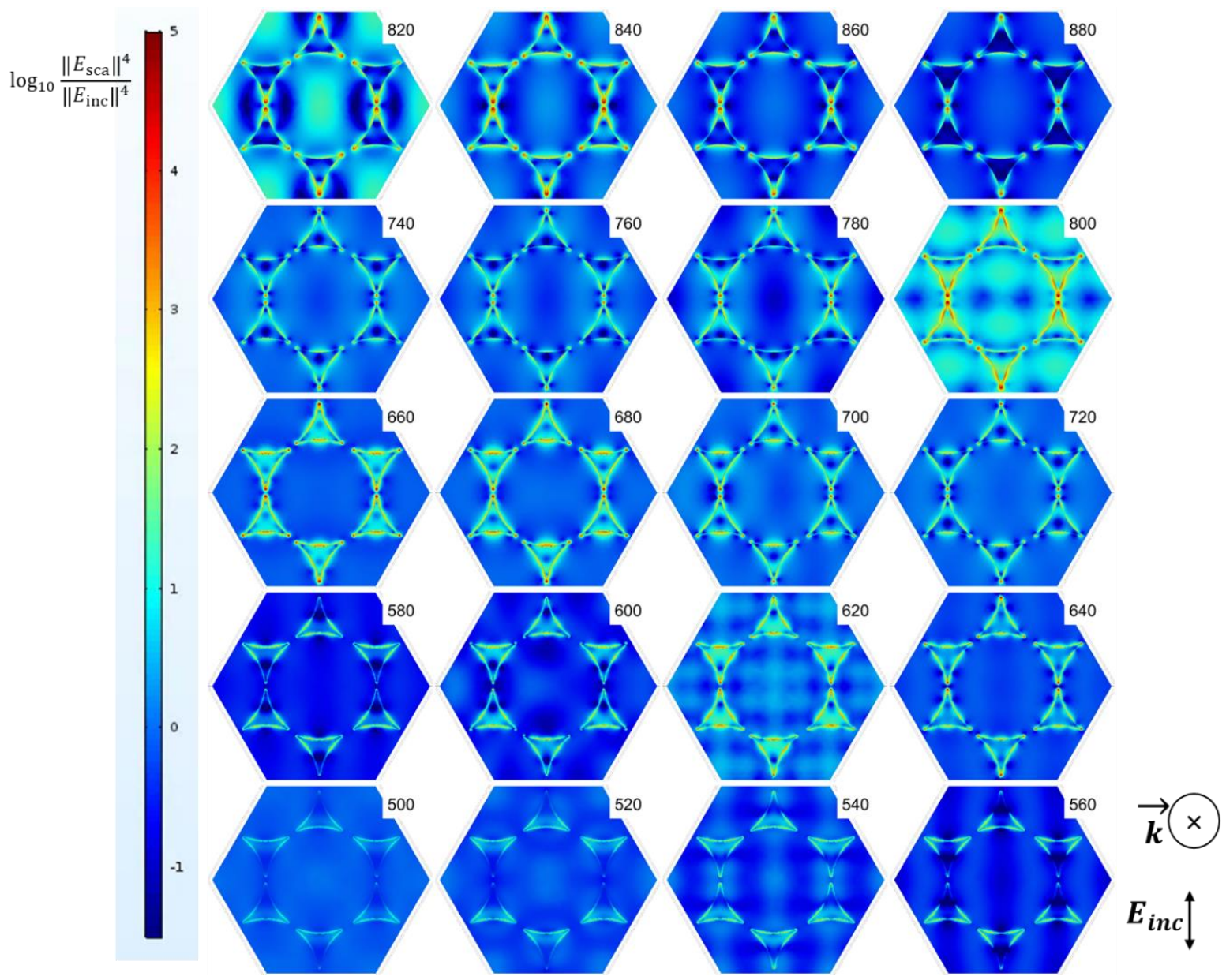

Figure 3-28 $\boldsymbol{e}_{n s l}^{c o m p}$ for an infinite array of periodic nanoparticles for wavelength range $500 \mathrm{~nm}-880 \mathrm{~nm}$.

Figure 3-29 shows the maximum and integrated enhancement factors for single particle and periodic array simulations. The maximum enhancement factor for the periodic array was found to be 100 times larger than the single particle simulation. The maximum integrated enhancement factor was found to be 2.5 times larger than the single particle simulation. 

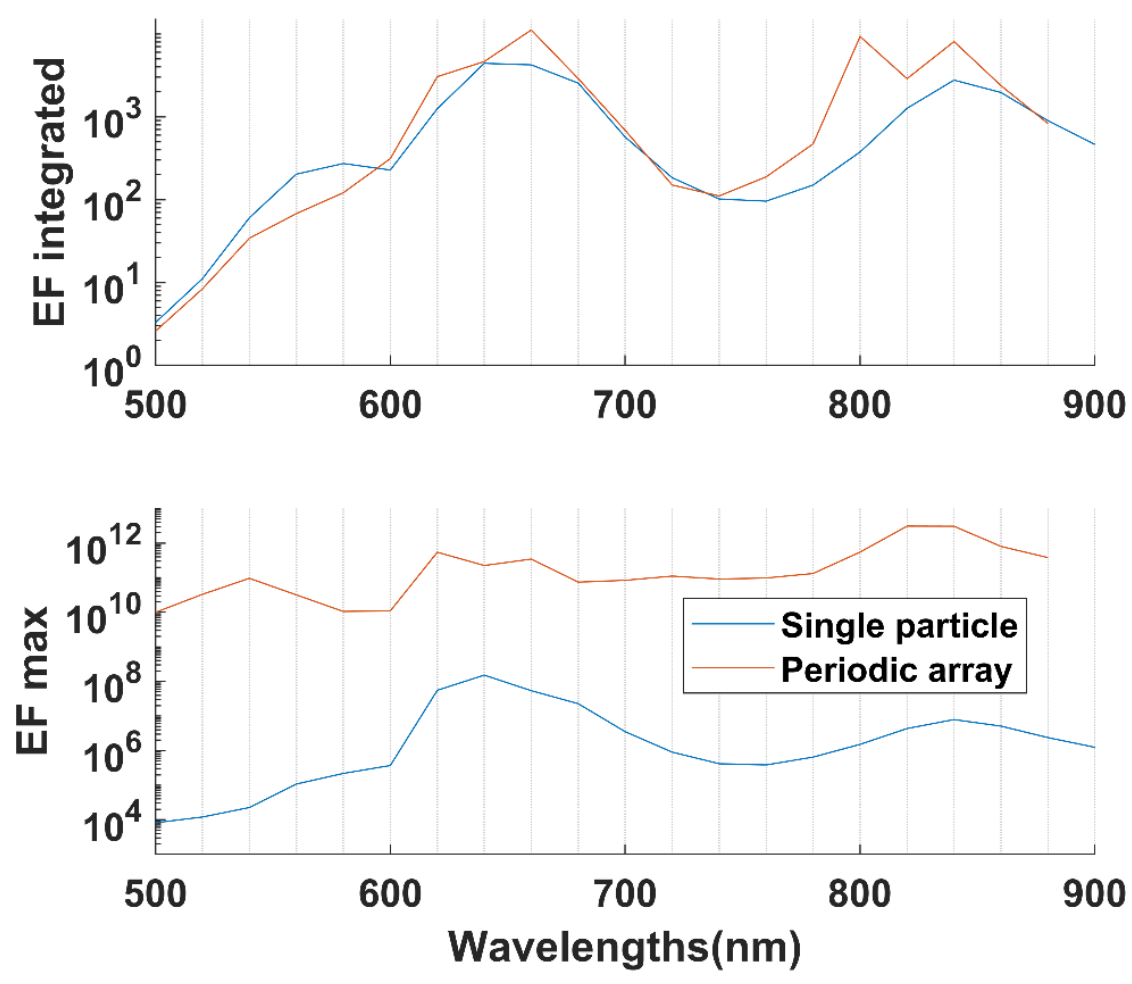

Figure 3-29 Integrated and maximum electric field enhancement factors for single particle and periodic array simulations.

\subsubsection{Nanoporous simulations}

\subsubsection{Electromagnetic enhancement for NS-7 substrates}

Figure 3-30 shows the theoretical electric field enhancements obtained from numerical modeling. The thickness was varied from $5 \mathrm{~nm}$ to $80 \mathrm{~nm}$ in steps of $5 \mathrm{~nm}$. The cell modeled was $200 \mathrm{~nm} \times$ $200 \mathrm{~nm}$ and the direction of propagation and polarization of the applied electric field are also shown in the figure. The electric fields are plotted on a surface located $0.1 \mathrm{~nm}$ above the top surface of the nanoporous structure to avoid the boundary of gold and air. The enhancements were found to be larger for layer thicknesses less than $20 \mathrm{~nm}$ after which the values diminished as were found to level out. 


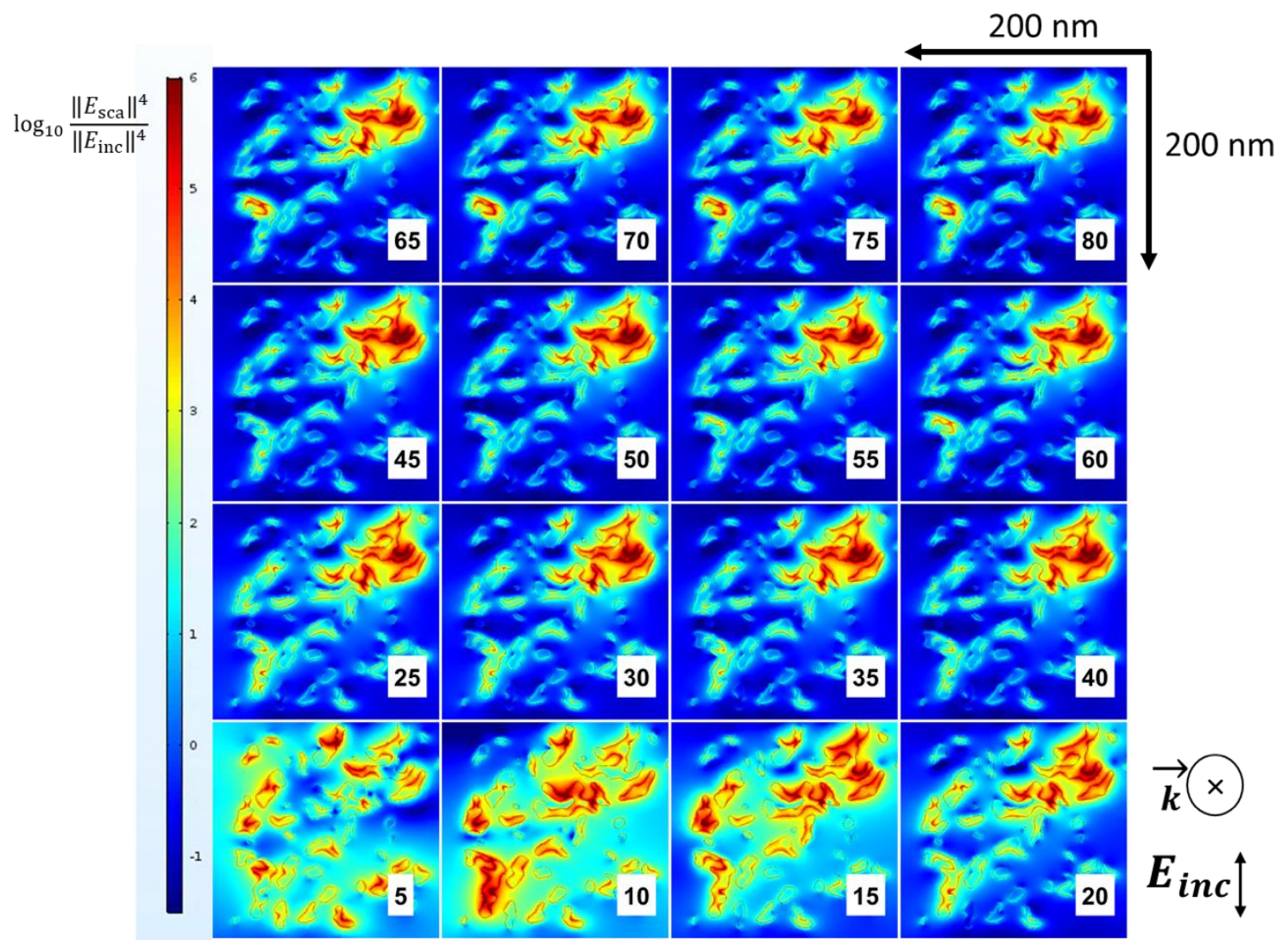

Figure 3-30 $\boldsymbol{e}_{n s l}^{\text {comp }}$ for NS-7 substrates for different layer thicknesses (5 nm-80 nm). The enhancement increases slightly with layer thickness and then gradually decreases for increased layer thicknesses.

\subsubsection{Electromagnetic enhancements for NS-17 substrates}

Figure 3-31 depict the theoretical electromagnetic enhancements for NS-17 substrates. The plane depicted in the images was selected from $0.1 \mathrm{~nm}$ above the top surface of the gold layer to avoid field discontinuities at the interface between the gold layer and air. For larger thickness of gold layers, NS-17 substrates depicted more location with hot-spots (reddish regions in the image) when compared to NS-7 substrates. 


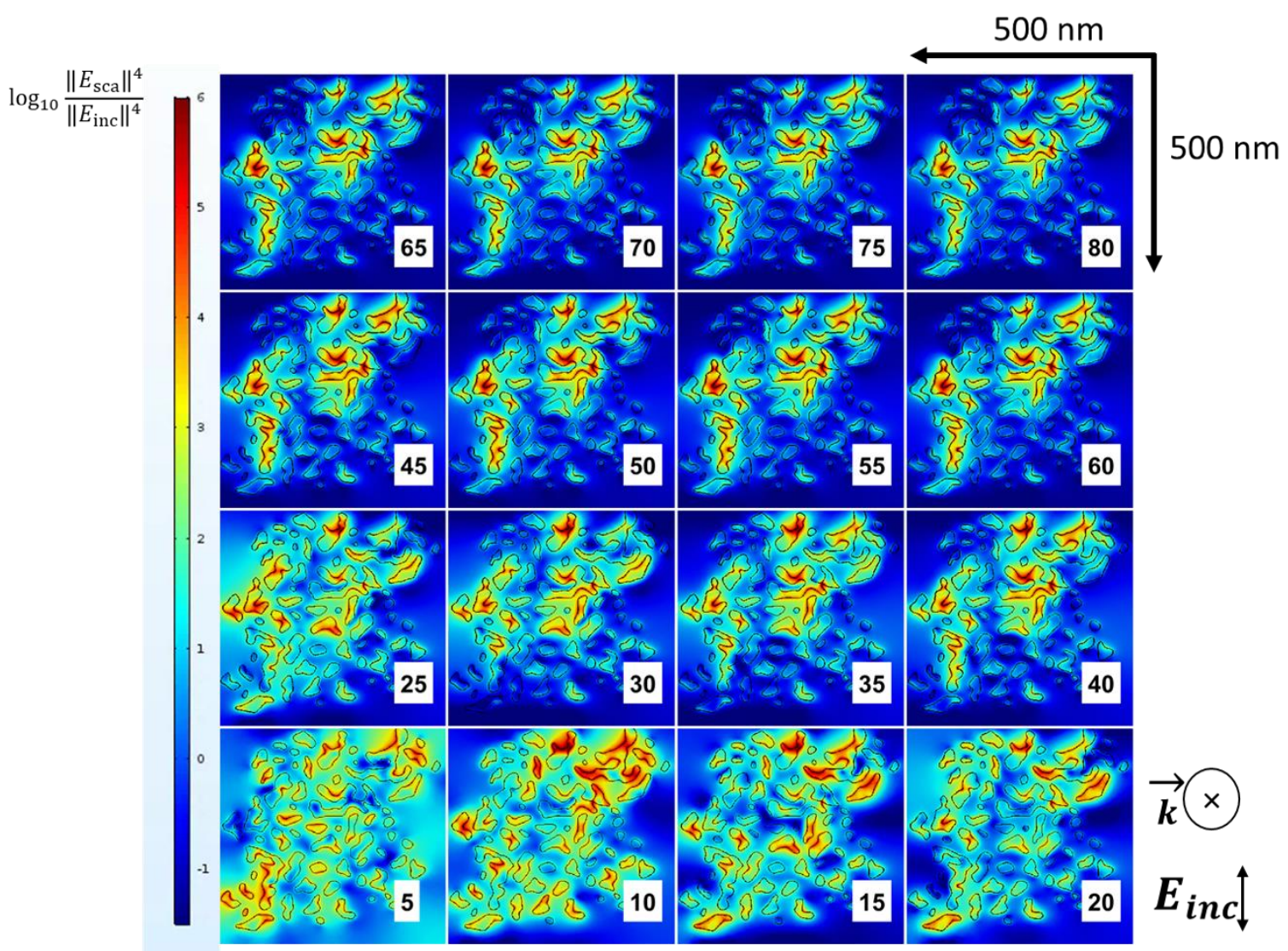

Figure 3-31 $\boldsymbol{e}_{\boldsymbol{n} \boldsymbol{s l}}^{\boldsymbol{c o m p}}$ for NS-17 substrates for different layer thicknesses (5 $\mathrm{nm}-80 \mathrm{~nm}$ ). The enhancement increases with increasing thickness and then start gradually decreasing.

The simulation geometry for NS-7 substrates was shorter than the NS-17 substrates as the AFM image of NS-17 substrate was used to create NS-7 substrates. This was done as the quality of image obtained for NS-7 substrates through AFM did not allow for proper digitization. To compute average enhancement factors for the substrate, surface and volume integrations were performed. Since the nanoporous structure had sharp edges, integrated electric field and enhancement factors provided a better comparison for both substrates.

The integrated field was defined as

$$
E_{\text {porous }}^{\text {int }}=\frac{\iiint \boldsymbol{E}_{\text {sca }} d x d y d z}{V}
$$

where $\mathrm{V}$ is the volume defined by the shaded region in Figure 3-32A. 
The integrated enhancement factor is defined as

$$
e_{\text {porous }}^{\text {comp }}=\frac{\iint\left|\boldsymbol{E}_{\text {sca }}\right|^{4} d x d y / \iint\left|\boldsymbol{E}_{\text {inc }}\right|^{4} d x d y}{A}
$$

Where A is the surface area defined by the shaded region in Figure 3-32 B.

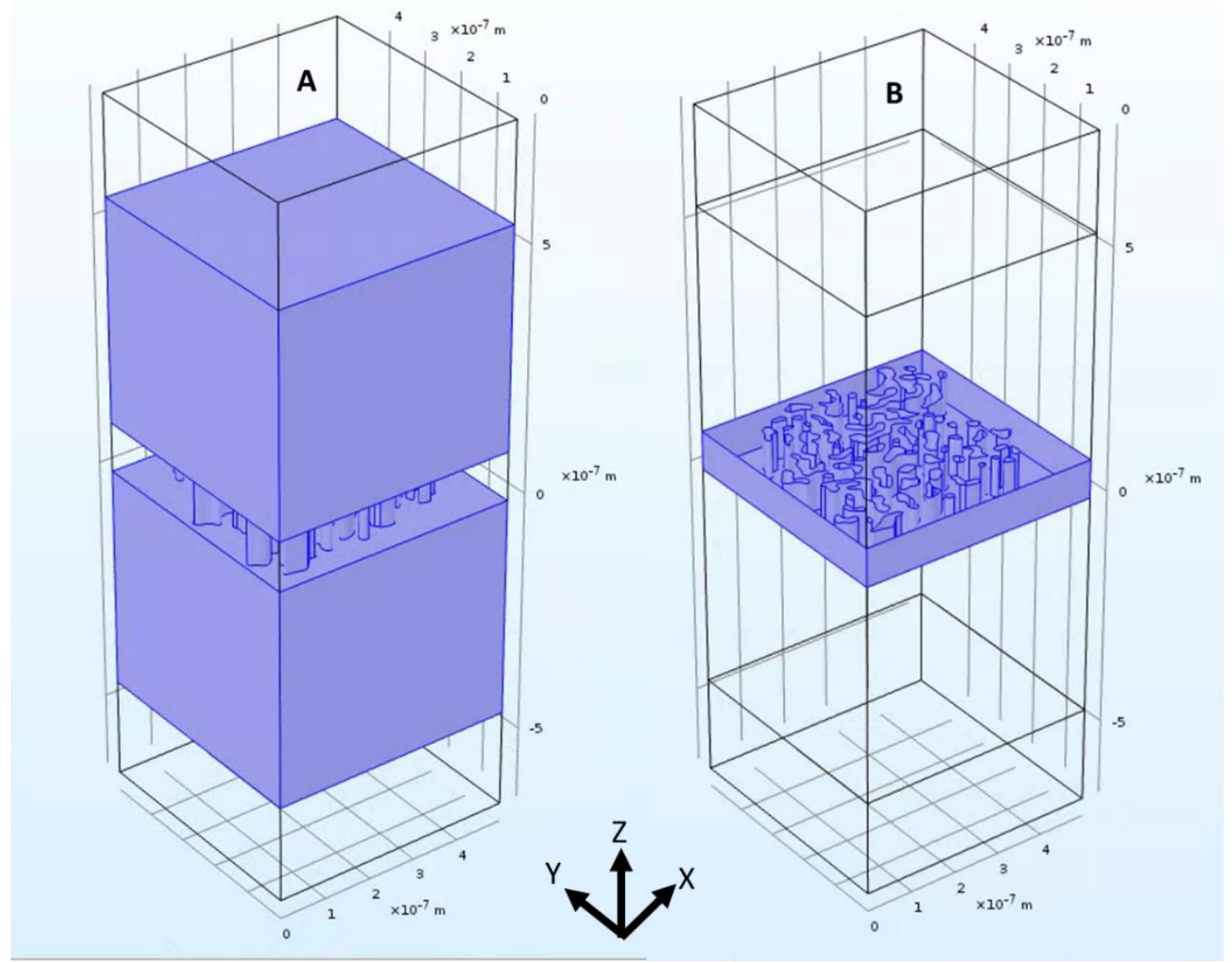

Figure 3-32 A) The blue shaded region used for volume integration. B) The blue shaded region used for surface integration of NS-7 and NS-17 substrates.

The integrated electric field is depicted in Figure 3-33 for both the substrates. The peak integrated electric field for NS-7 substrates was found to be around $10 \mathrm{~nm}$ thickness and for NS-17 it was found to be around $25 \mathrm{~nm}$ thickness. 


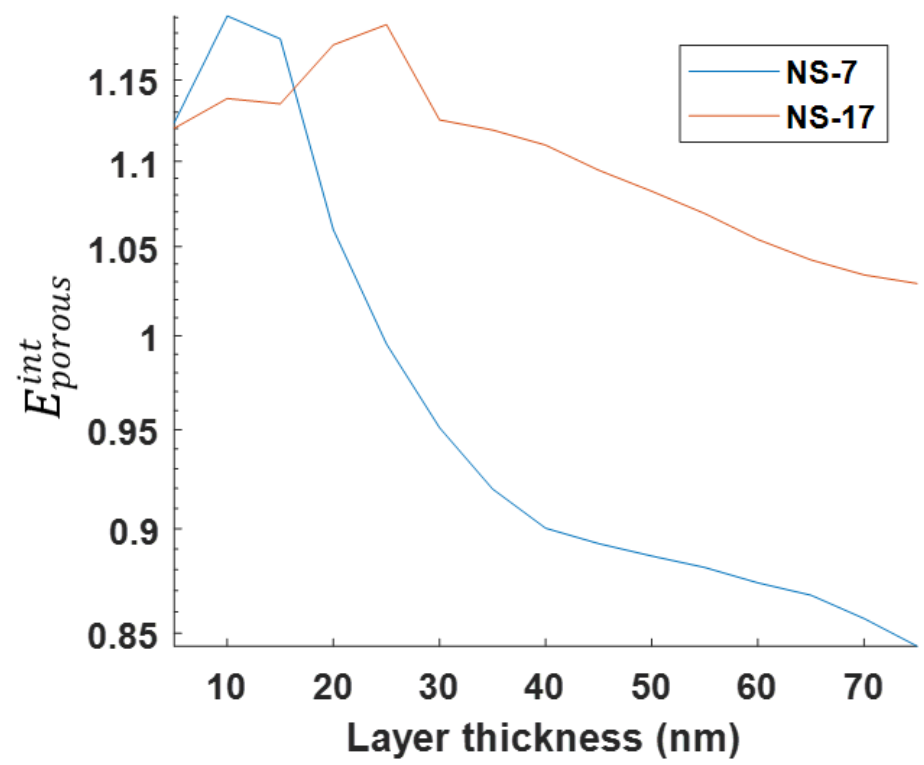

Figure 3-33 Integrated E field ( $\boldsymbol{E}_{\text {porous }}^{\text {int }}$ ) for NS-7 and NS-17 substrates.

The enhancement factors normalised by the surface area of the nanoporous film are presented in Figure 3-34. The maximum enhancement factor obtained for both substrates was at $10 \mathrm{~nm}$ thickness of nanoporous film. The NS-7 substrates show larger enhancements than NS-17 substrates. At maximum enhancement, NS-7 substrates were found to have 2.1 times larger enhancements compared to NS-17 substrates. The trends observed in the theoretical simulations show that increasing layer thickness increased the enhancements and reached a maximum at 10 nm for both NS-7 and NS-17 substrates. For experimental observations, the largest enhancement was observed for $25 \mathrm{~nm}$ for NS-7 substrates and for NS-17 substrates. This was likely due to the simplified approximation the nanoporous layers. The simplified geometry of the nanoporous glass suitably depicted the trend of enhancements with increasing layer thickness. Volume integral of the scattered electric field showed peaks in the locations close to where peaks were observed in the experiments (Figure 3-33 and Figure 3-22A). Since the porous structure represents a volume where molecules can interact with the substrates, better 3D porous structure needs to be designed for accurate modeling. The pore size of the nanoporous films were the same for all heights in the simulation but this is not the case for experiments. The pore-filling dynamics must be further evaluated to perform more accurate simulations. 


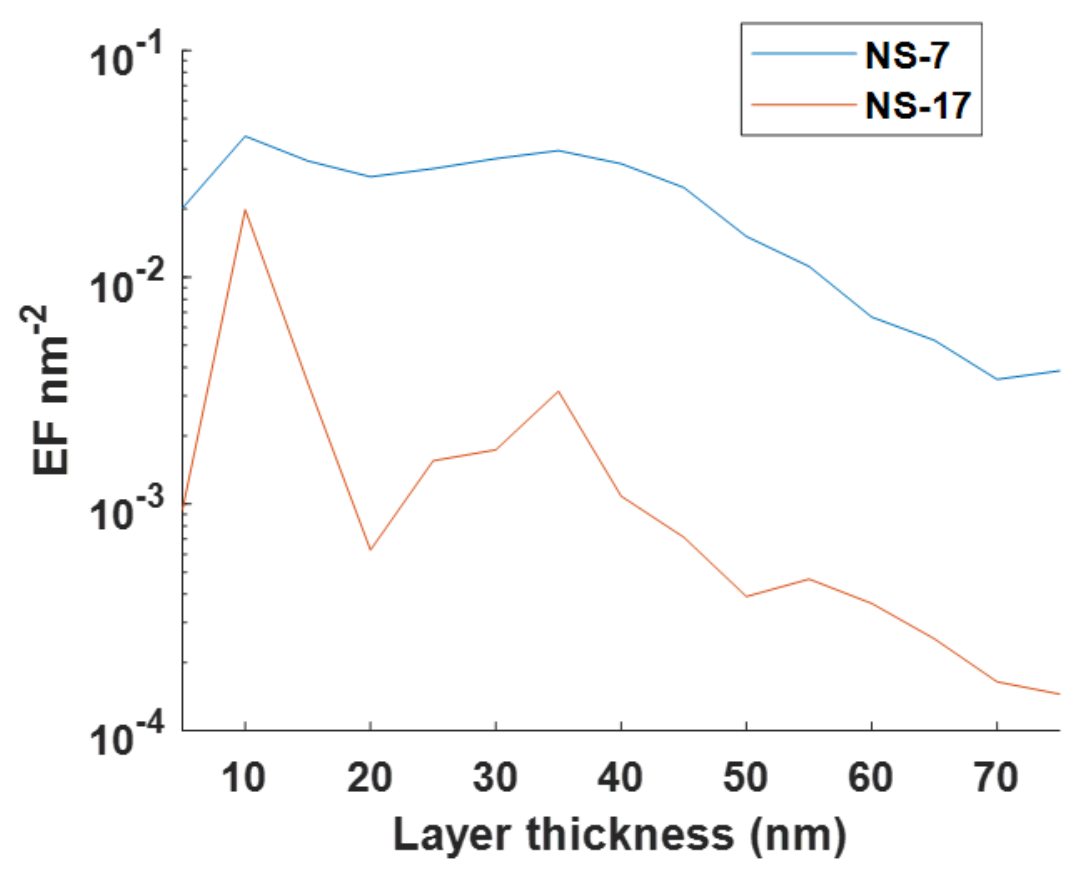

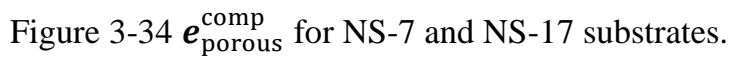

\subsection{Clinical applications}

\subsubsection{Ex-vivo data collection}

Raman spectra were collected from ex-vivo tissues excised during full breast mastectomy. The data was collected within 45 minutes of excision. The whole excised breast tissue was placed on a flat plate attached to a translation stage. The tissue was sliced open to expose the tumor surface for collection of Raman spectra as shown in Figure 3-35. The dark regions within the yellow ellipse indicated regions of tumor and data was collected from within the tumor region and an adjacent healthy tissue region. Optimal focusing was ascertained by using a low integration time to obtain best Raman peaks while translating the tissue. The final integration time for tissues was in the range of 40s-60s. The power of laser at the tissue was measured to be $15 \mathrm{~mW}$. The data collected was normalized by the laser power and general background correction was performed. The raw spectra and differences between the normal and tumor tissues are presented in Figure 3-36. The peaks marked in the background corrected data correspond to peaks of interest that represent vibration modes of biomolecules. Strong fluorescence was observed from the tissues. Most of the breast tissue contains collagen. The peaks obtained were due to a combination of lipids and proteins such as valine, glycine, proline and phenylalanine (components of collagen). Data from 
another patient are also presented in Figure 3-36. The differences in the normal tissue and tumor tissues were prominent for this patient. Peaks for the normal tissues were well defined but they were absent in tumor tissues. The variability in tissues for different patients was found to be significant and due to the usage of patent blue dye for assessing lymph node metastases, further data are planned to be acquired.

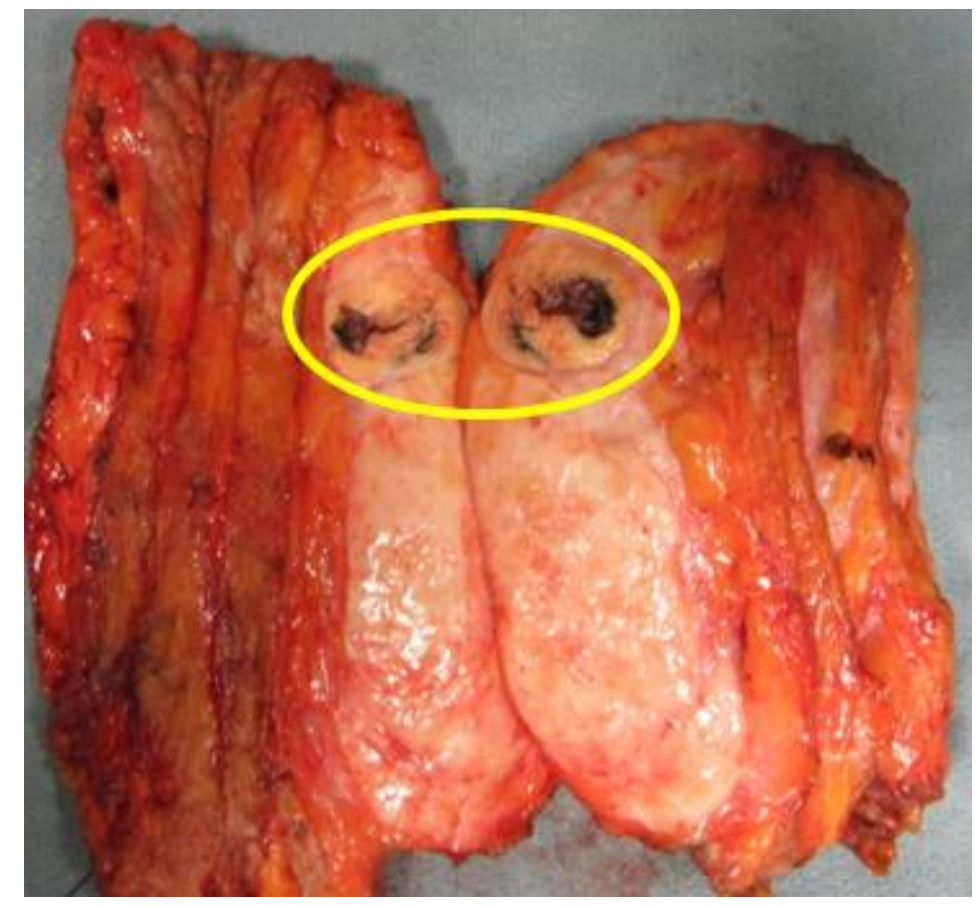

Figure 3-35 Photographic image of excised breast tissue. 

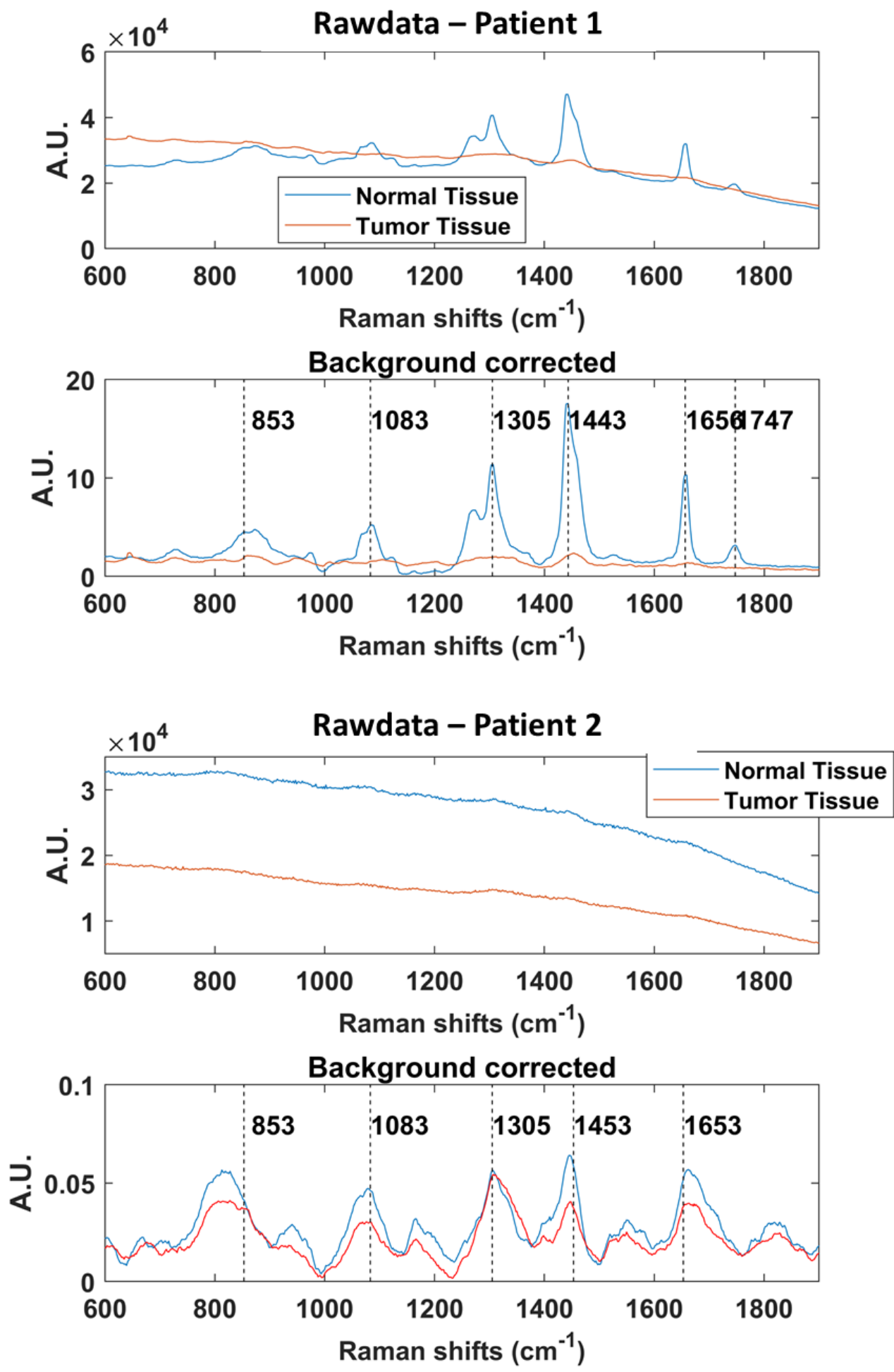

Figure 3-36 Raman spectra collected from excised breast tissues for patients. 


\subsubsection{Comparison of data collection from ex-vivo and processed tissues}

Formalin fixed paraffin embedded tissues are usually stored after collection of biopsy. The paraffin embedded tissues cassettes were used to create $20 \mu \mathrm{m}$ thick sections of tissues. The sections were placed on microscopic cover slips $(22 \mathrm{~mm} \times 22 \mathrm{~mm} \times 0.17 \mathrm{~mm})$. These tissues were processed to remove paraffin using a standard paraffing washing process using multiple washes using xylene followed by rehydration of tissues using ethanol. Data collection was immediately performed on these tissues on a confocal Raman microscope (inVia Confocal Raman Spectrometer, Renishaw, UK). The spectra obtained from a confocal Raman system depicts a mixture of tissue and paraffin signals (Figure 3-37). The sharp peaks located at $1060 \mathrm{~cm}^{-1}, 1134 \mathrm{~cm}^{-1}, 1172 \mathrm{~cm}^{-1}, 1294 \mathrm{~cm}^{-1}$ for the processed tissues are due to the contributions from paraffin wax [137] and are absent from the ex-vivo breast tissue. The differences between the normal and tumor tissues cannot be justified by univariate analysis and hence a biochemical model based on multivariate analysis is required to observe the variation in normal and tumor tissues.
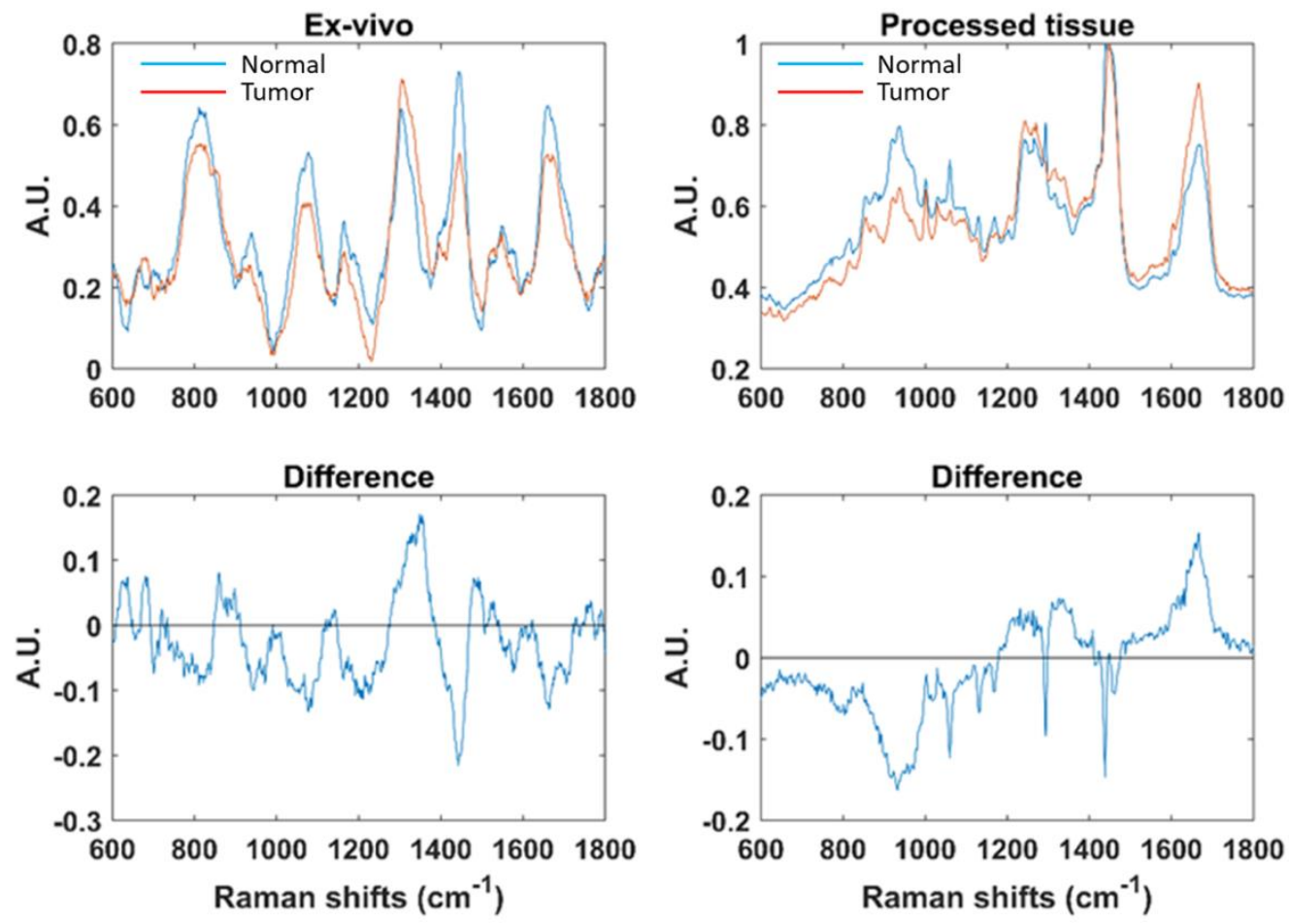

Figure 3-37 Comparison of spectral data collection from ex-vivo and processed tissues. The ex-vivo data collection was done using a custom Raman setup and a confocal microscope was used to collect data from processed tissues. 


\section{Summary and Conclusions}

NSL was used to create and characterize nanoparticles on flat substrates. Absorption measurements were performed on flat substrates to obtain extinction spectrum of the nanoparticles created. Disposable SERS fiber probes were fabricated using the NSL technique. Increasing the NA of the fibers was found to improve the quality of spectra collected. Direct measurements show similar SERS performance of the optical fibers for all NAs whereas remote measurements show that larger NA is required to obtain improved Raman characterization. This indicates that such SERS fibers can be employed for remote sensing applications such as endoscopy. A simple iterative matching algorithm with non-negative constraints was sufficient for removing the fluorescence background from the optical fibers allowing for better visualization of spectral peaks. Characterization of SERS optical fibers showed that 0.5 NA had same performance $(\approx 98 \%)$ in direct/remote configurations and had the largest enhancement factors demonstrating advantages of using a high NA optical fiber for SERS measurements. A simple and inexpensive Raman collection setup can be utilized with the SERS fiber-optic substrates to be used for remote sensing. Crystal violet $(\mathrm{CV})$ dye was used for proof of concept to observe the performance of SERS fiber probes in a remote sensing configuration. Further experiments should be performed to optimize NSL process and evaluate the limit of detection. Moreover, this platform can be optimized by changing the size of microspheres for NSL as well as using advanced etching techniques creating nanoporous materials. Via using NSL, bare optical fibers with SERS tips can be easily fabricated and employed for remote sensing. It is envisioned that these SERS fibers can be readily used in miniature environments such as microfluidic devices or microendoscopy to perform SERS sensing. They also allow for temporary introduction of nanoparticles in a medium for quick qualitative evaluation.

Two types of nanoporous substrates were fabricated, and their SERS activity was evaluated. For the AuNS-7 substrates, the visibility of nanoporous pattern diminished rapidly with increased thickness of Au coating when compared to AuNS-17 substrates. SERS activity rapidly dropped for AuNS-7 when thickness of Au increased from $25 \mathrm{~nm}$ to $50 \mathrm{~nm}$. This was likely due to the disruption of the nanoporous structure for thicker Au layers as shown in the AFM images. The AuNS-7 substrates exhibited larger SERS activity due to a higher density of hot-spots because of the smaller pore sizes and reduced air gap between the pore edges. Further control of the layer 
thickness is required to establish pore filling dynamics and its corresponding effect on the SERS activity. AuNS-7 substrates had four times higher SERS enhancements than the AuNS-17 substrates. AuNS proved to be mechanically robust and disposable SERS substrates.

Extinction spectrum obtained from numerical modeling of single particles matched the absorbance spectrum and the primary resonance peak was located at $660 \mathrm{~nm}$ compared to the experimental peak at $622 \mathrm{~nm}$. Experimentally obtained spectra were found to be slightly blue shifted compared to the theoretically obtained spectrum. This difference was likely due to the variances in optical properties and nanoparticle height disparities between experimental setup and simulations. The secondary peak in the simulation located at $840 \mathrm{~nm}$ was absent in the experimental data. The average and integrated SERS enhancement factors were computed for single particle and a periodic array of particles. The maximum enhancement factor for the periodic array was found to be 100 times larger than the single particle simulation. The maximum integrated enhancement factor was found to be two and a half times larger than the factor for single particle simulation. Simulation of nanoporous films results in 2.1 times larger enhancements for NS-7 substrates when compared to the NS-17 substrates. Experimentally, NS-7 substrates were found to produce 3.6 times larger enhancements.

Preliminary clinical data show that there are differences between cancerous regions and healthy regions for breast tissues. The differences obtained were visually examined and further multivariate analysis is required for differentiating healthy and diseased regions.

Fiber optic SERS substrates can have a lot of potential industrial and clinical uses. Since the tips contain the nanoparticles, they can be temporarily introduced in a sensing environment to enhance the Raman detection. The substrates created in this work are capable of being used as fiber-optic patch cables which can be attached to any of the commercially available sensing probes. The intensity enhancements obtained can be further improved by optimization (as shown by theoretical simulations) and using a high NA optical fiber arrangement. Unfiltered fiber optic cables can be used for sensing making it possible to reduce the size of the probes which can be suitable for interstitial applications. The major challenge lies in making the nanoparticles attach firmly to the fiber optic tips as mechanical scraping can peel them off. Encapsulating the nanoparticles in another layer of silica or alumina can be done to make them mechanically robust. 
The surface area of sensing using NSL is less than the surface area of sensing achieved through NS-7 and NS-17 substrates. This resulted in larger enhancements for nanoporous substrates than NSL substrates. Since the fiber optic cables can be made from borosilicate glass, the same approach that was used to create the nanoporous substrates can be used to create nanoporous fiber optic tips. This can be done to achieve a larger sensing area for fiber-optic substrates. 


\section{Future work}

\subsection{Improving 3D nanoporous SERS substrates.}

This study utilized Au layer coating but further extension of this study using the AuNS substrates decorated with $\mathrm{Au} / \mathrm{Ag}$ nanoparticles is planned to fabricate 3D SERS active substrates with higher enhancement factors. The large specific surface area of the NS substrates can be explored to accumulate SERS from a large number of molecules. The possibility of impregnating the substrates with a variety of media to control the dielectric properties surrounding the plasmonic structures that can be localized on the inner walls of the pores can be explored.

\subsection{Film growth on nanoporous structures.}

The pore filling dynamics of the nanoporous films can be further studied by theoretical evaluation of the deposition of Au. Kinetic Monte-Carlo method can be used to perform simulation of film growths on nanoporous structures [138]. It allows to use available AFM maps as a base geometry to perform the film growth. By understanding the dynamics and through advanced deposition techniques, the nanoporous SERS sensing can be further optimized. Angular deposition of $\mathrm{Au}$ layers can be studied to evaluate the formation new nanopatterns starting from the nanoporous pattern of the NS glasses. Various nanoparticles can also be embedded inside the nanopores and can be used as seed for nucleated film growth.

\subsection{Nanoporous fiber-optic SERS substrates}

Optical fiber substrates can be produced using Borosilicate glass with composition suitable to create nanoporous structures. The tip of an optical fiber can be converted to a nanoporous structure allowing for a unique sensor with a high sensing area as show in Figure 5-1. The nanoporous tip can then be modified using nanoparticles to create not only plasmon based sensors but also other sensor that can utilize the larger surface area provided by the nanoporous structure. Since the structure is made through the extension of the fiber optic tip, it is expected to be highly mechanically stable and chemically inert unlike external structures attached to the fiber tip. Various pore sizes can be created along with optical fibers with varying diameters and NA. 


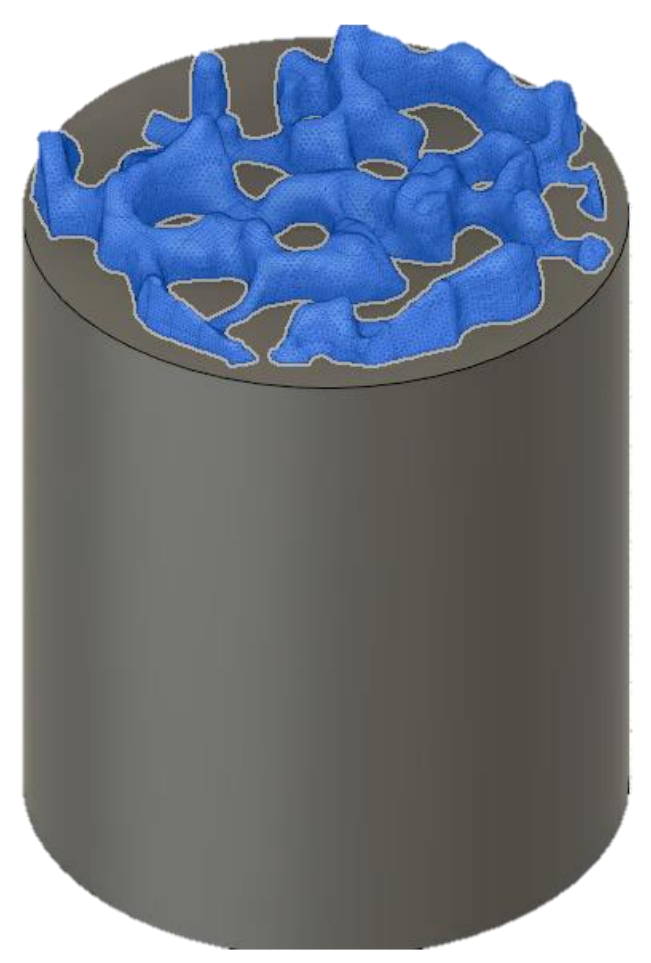

Figure 5-1 Optical fiber with nanoporous tips.

\subsection{Sapphire based fiber optic SERS substrates}

Silica optical fibers-based SERS substrates showed that SERS can be utilized to mitigate the fluorescence background of the optical fiber and can be used for remote sensing. Even though the background can be mitigated, silica fibers exhibit a large fluorescent background below $800 \mathrm{~cm}^{-1}$. The peaks in this region usually have a low SNR and this also limits the snapshot integration times due to the dynamic range limit of the instrument. It was observed that for the used excitation power, integration times of $1.5-2 \mathrm{~s}$ resulted in saturation of the spectrometer.

Sapphire usually produces less autofluorescence background than silica. Sapphire is a highly inert crystal which can be used in various environments. Sapphire based optical fibers generate a low background and present the opportunity of using sapphire peaks as an autocalibration method. Sapphire fibers from two manufacturers (denote Sa-F1 and Sa-F2) were tested for the background generation and compared with silica based optical fibers as shown in Figure 5-2. It was observed that Sa-F2 fiber resulted in the lowest fluorescent background and the sapphire peaks can be used 
for automatic wavelength calibration of the spectrometer. Moreover, they can also be used for evaluating reflected laser intensity variations for additional correction mechanism.
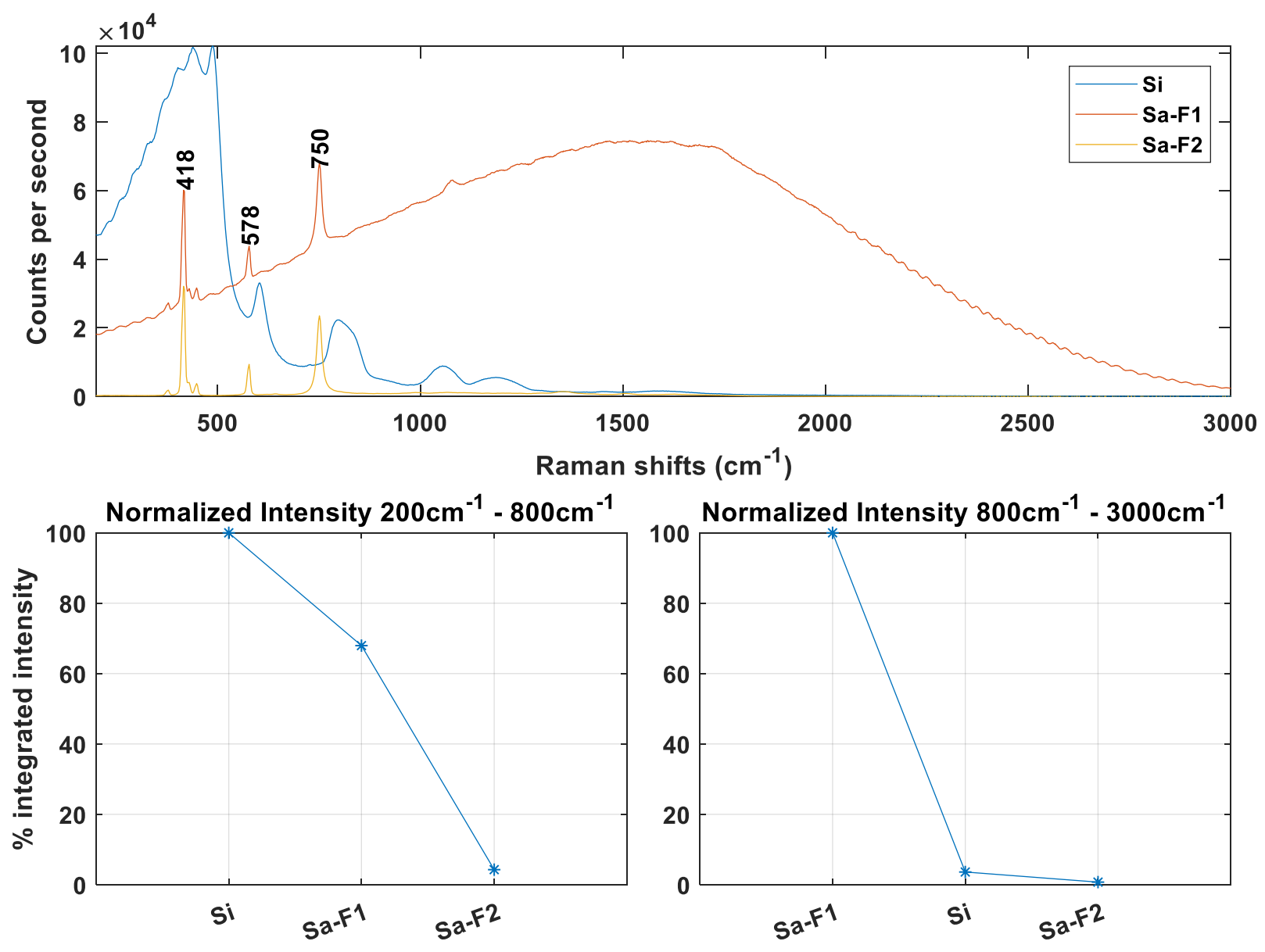

Figure 5-2 Comparison of background generated for silica and sapphire based optical fibers.

\subsection{Clinical study}

Spectra were obtained from tumor and healthy regions of excised breast specimens. The data showed high variability in obtaining the differences between tumor and healthy tissues. There was use of Patent blue dye to observe lymph node metastases and could have likely interfered with the measurements. Patent blue has Raman peaks in the regions around $1083 \mathrm{~cm}^{-1}, 1305 \mathrm{~cm}^{-1}$ and 1443 $\mathrm{cm}^{-1}$ which are the regions which interfere with data collected from tissues. Formalin fixed paraffin embedded tissues were sliced to $20 \mu \mathrm{m}$ thickness and deparaffinized. Spectra were collected from healthy and tumor tissues and it was observed that even after deparaffinization, residual peaks were obtained from paraffin. A confocal microscope had to be used for these 
measurements as the collection setup was not able to provide good SNR spectra for processed tissues.

Further clinical studies are planned for collection of spontaneous Raman spectra from excised breast specimens. The spectral library presented in Appendix I is planned to be used for creating classification models for diagnosing diseased conditions. Spectra will be collected from freshly excised ex-vivo tissues, FFPE embedded tissue blocks, cryo-frozen tissues and breast caner cell lines. A comparison of spectra is planned to be performed to evaluate the contributions of biomolecular markers at different scales. SERS optical fibers are planned to be used in bio-fluid analysis such as detection of glucose in urine or detection of drugs or other compounds in saliva. 


\section{Appendix I}

Spectra were collected from a set of biologically relevant biomolecules some of which can act as biomarkers for diseased conditions. All spectra were collected from pure powder obtained from Sigma Aldrich or Fischer Scientific. The spectra were normalized by the laser power and the integration time. For all the following spectra, the X-axis represents Raman shifts $\left(\mathrm{cm}^{-1}\right)$. 


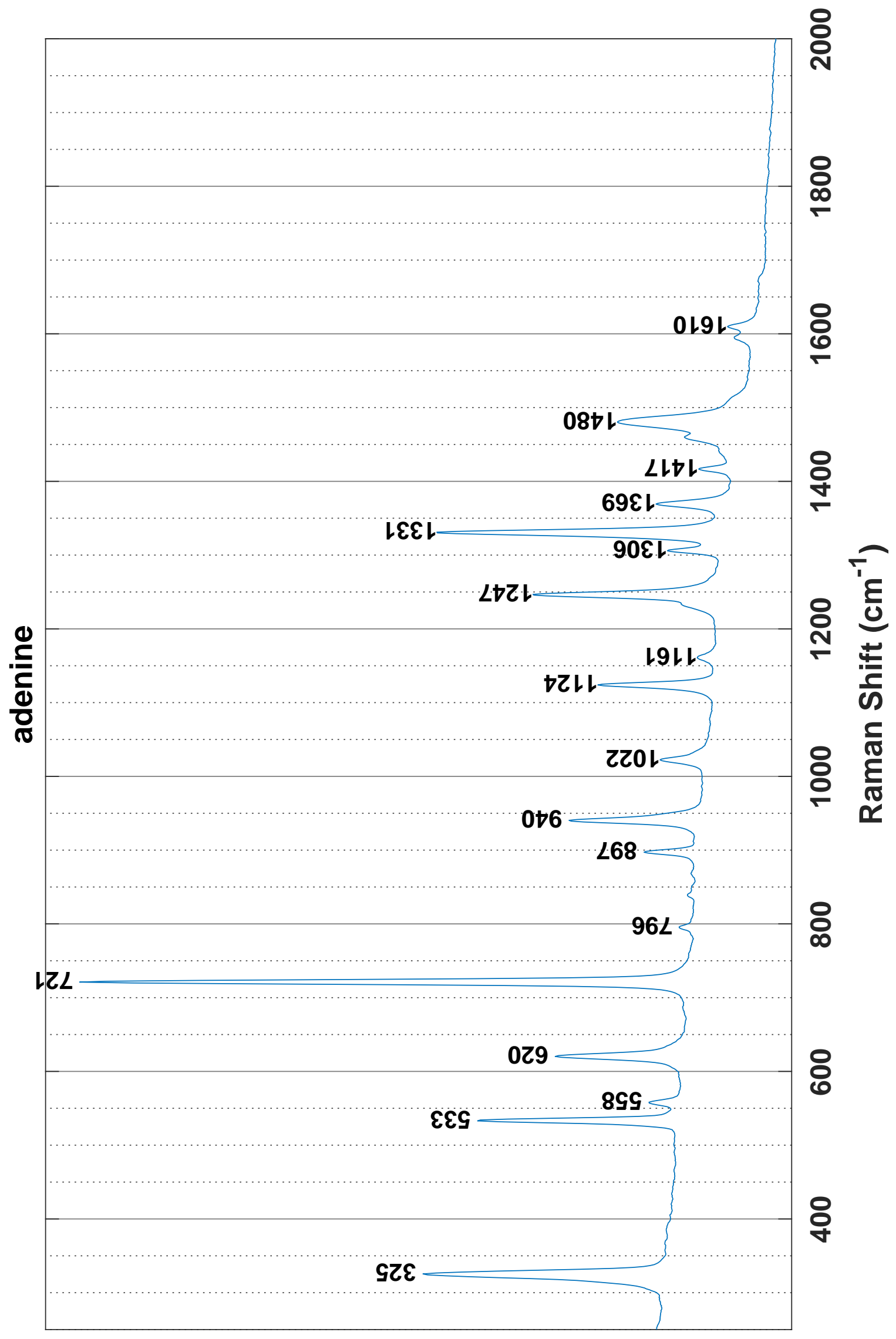




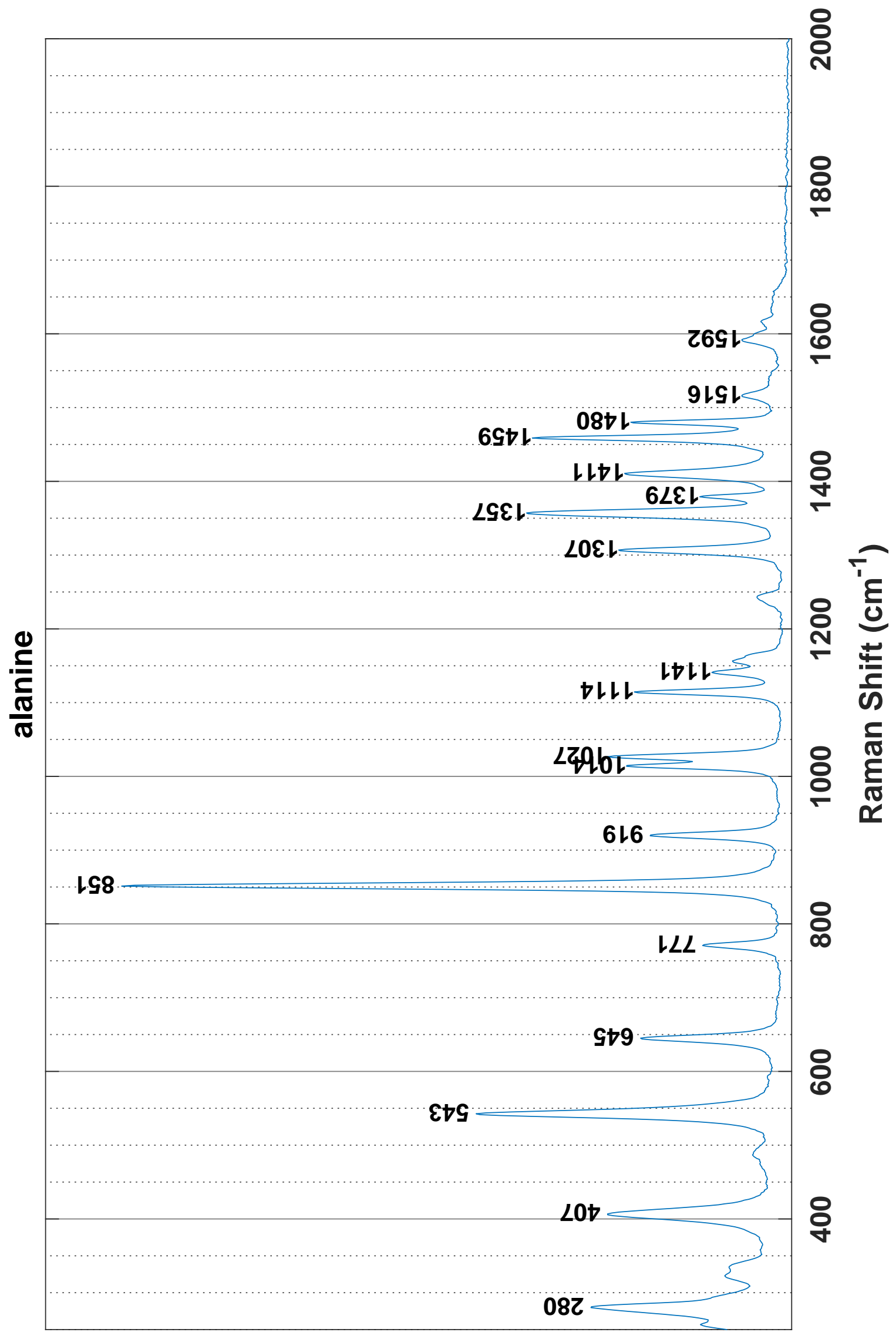




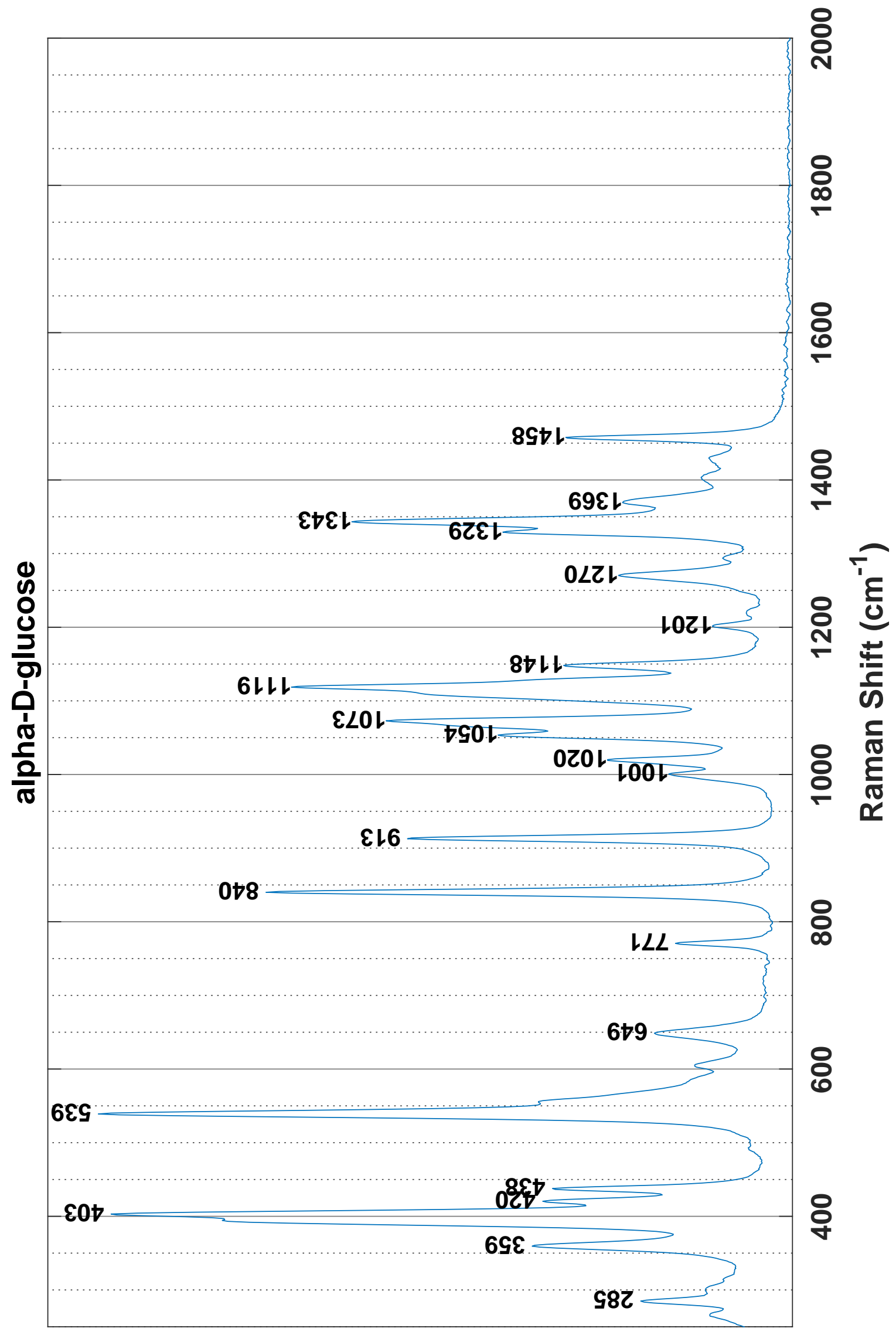




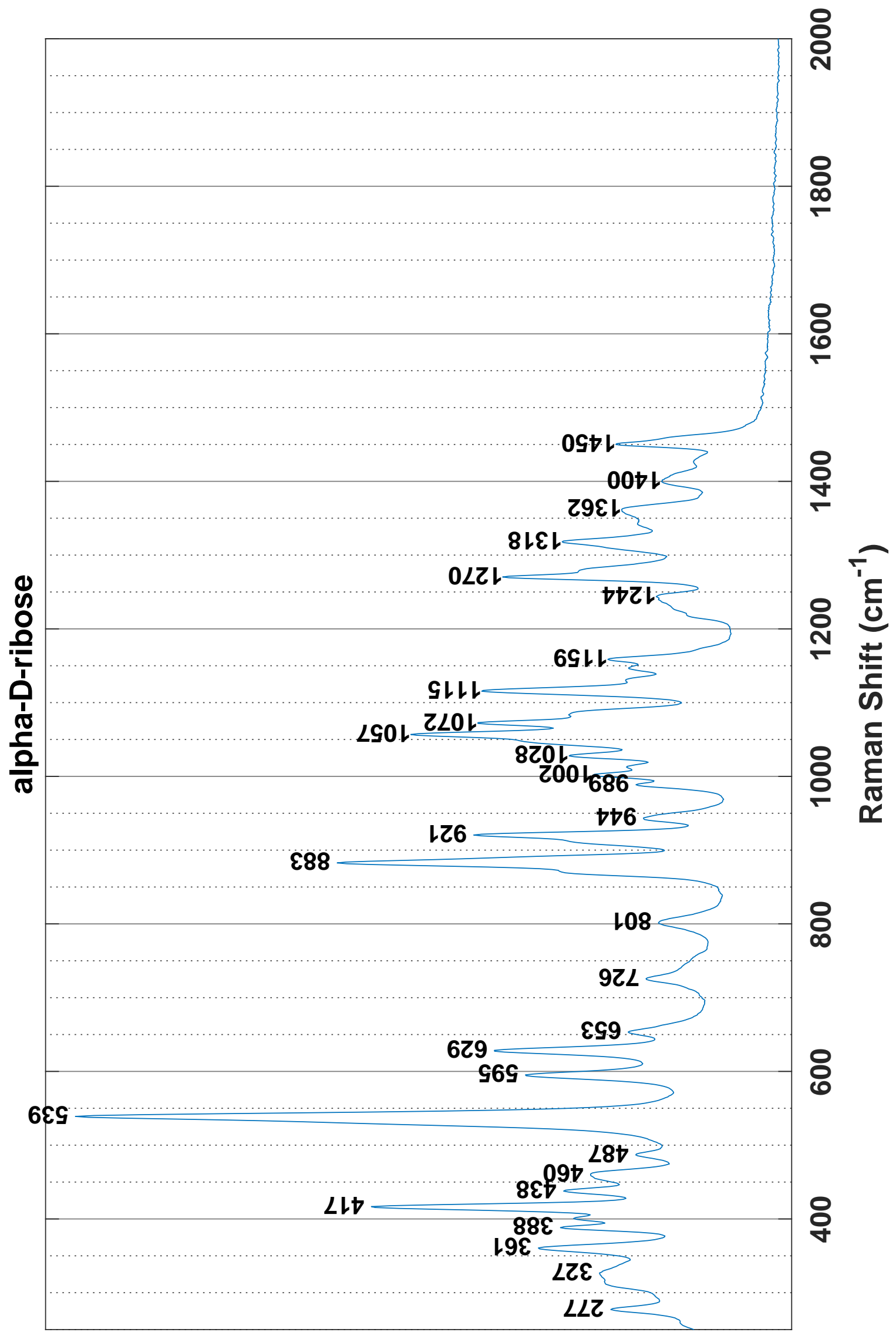




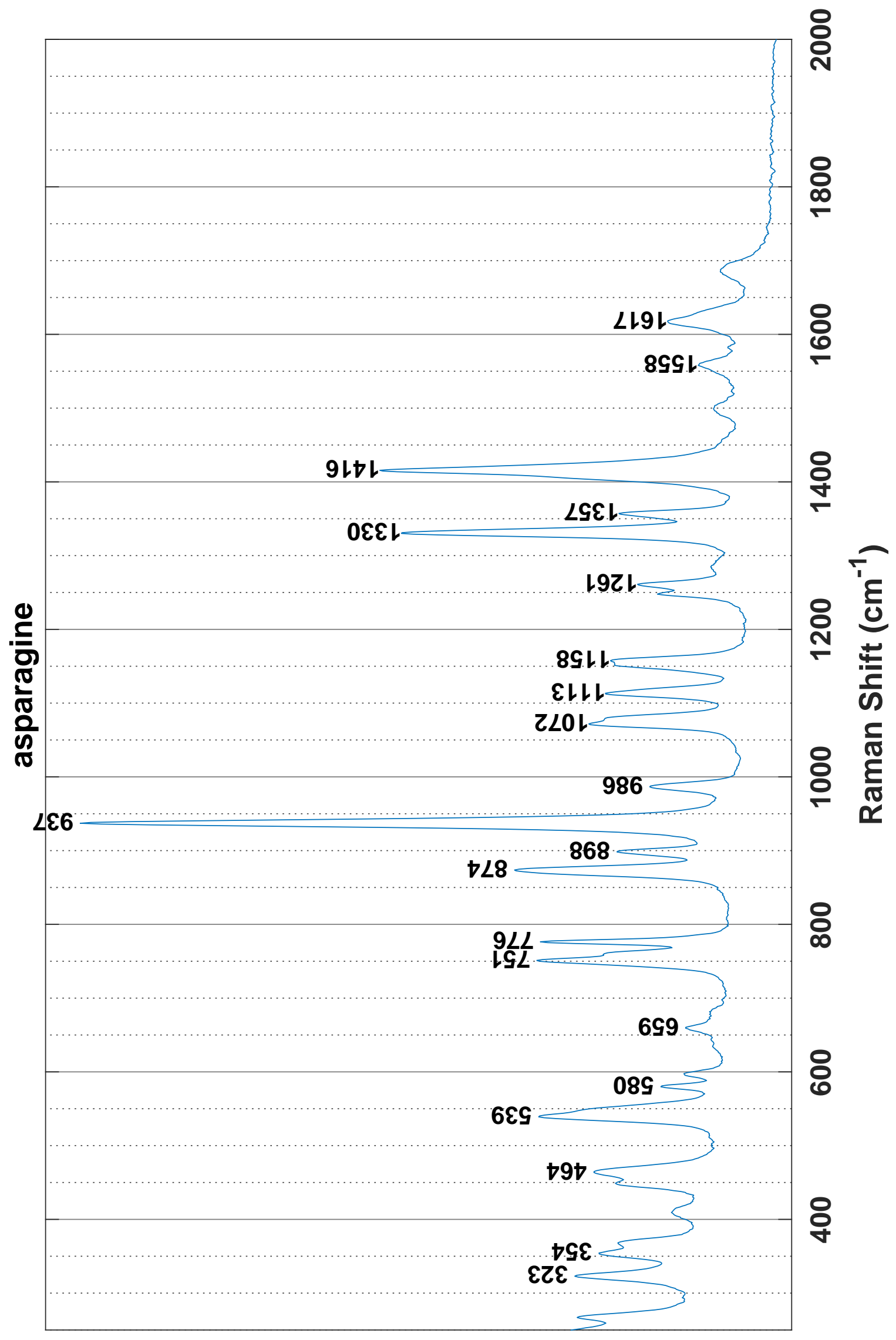




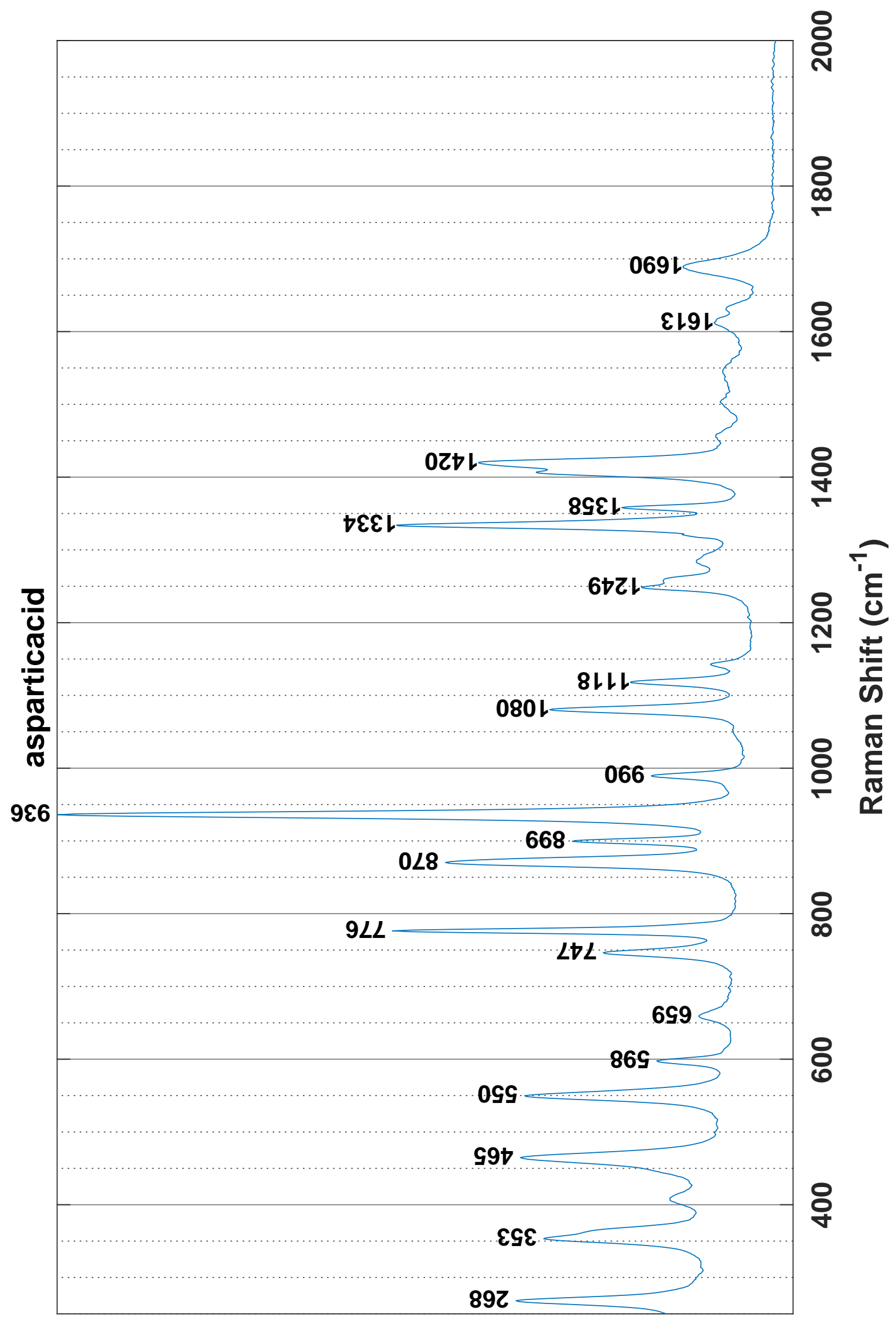




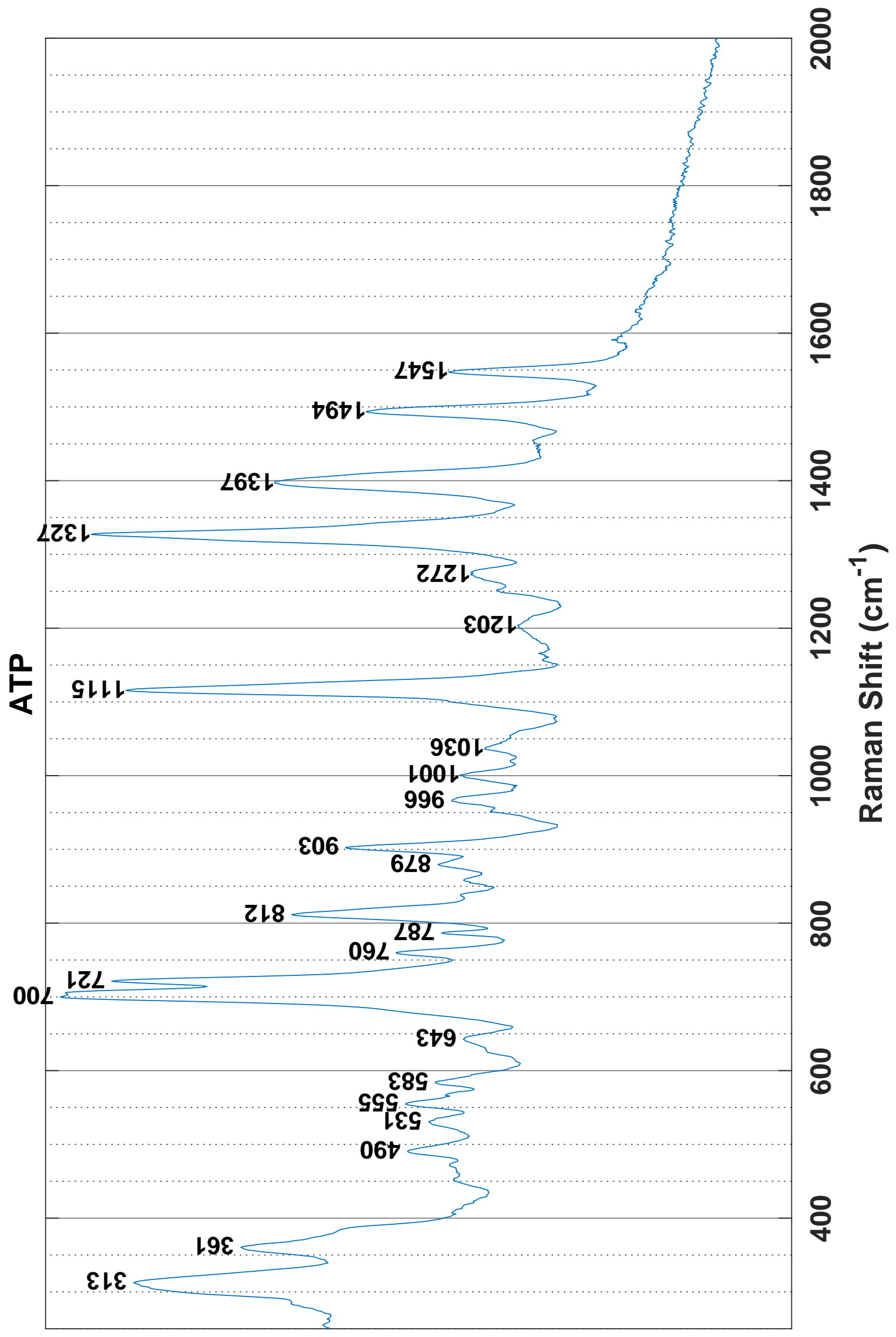




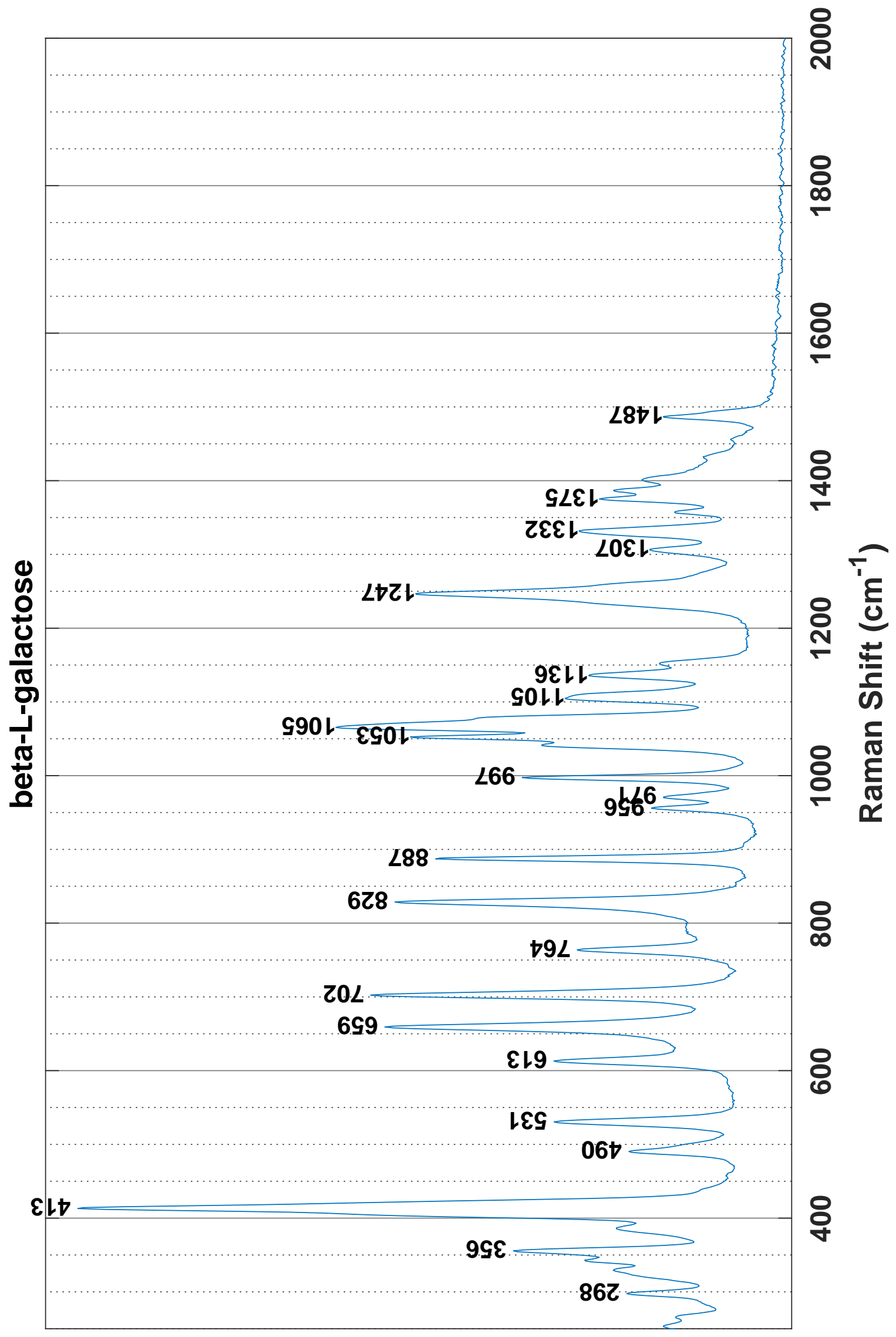




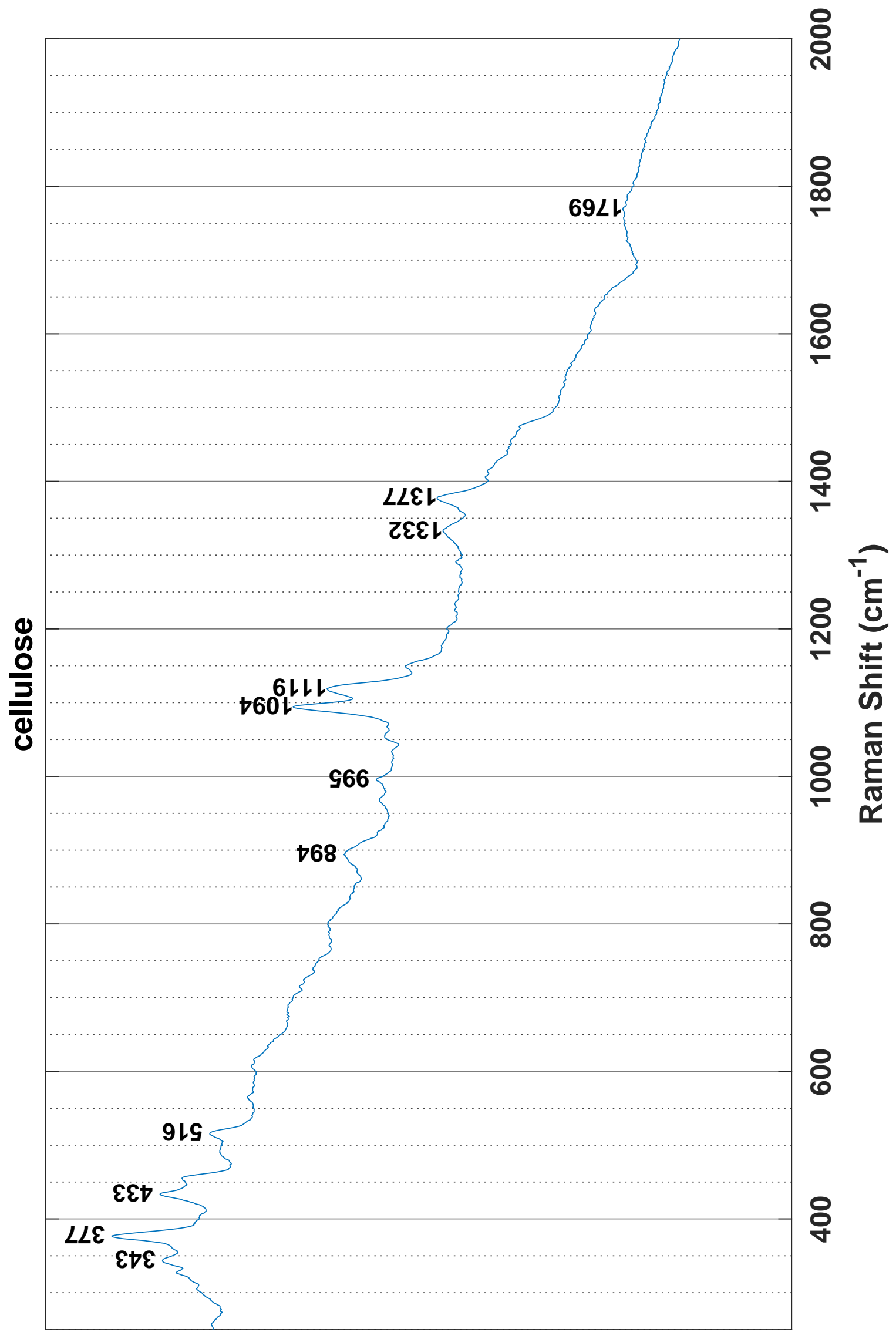




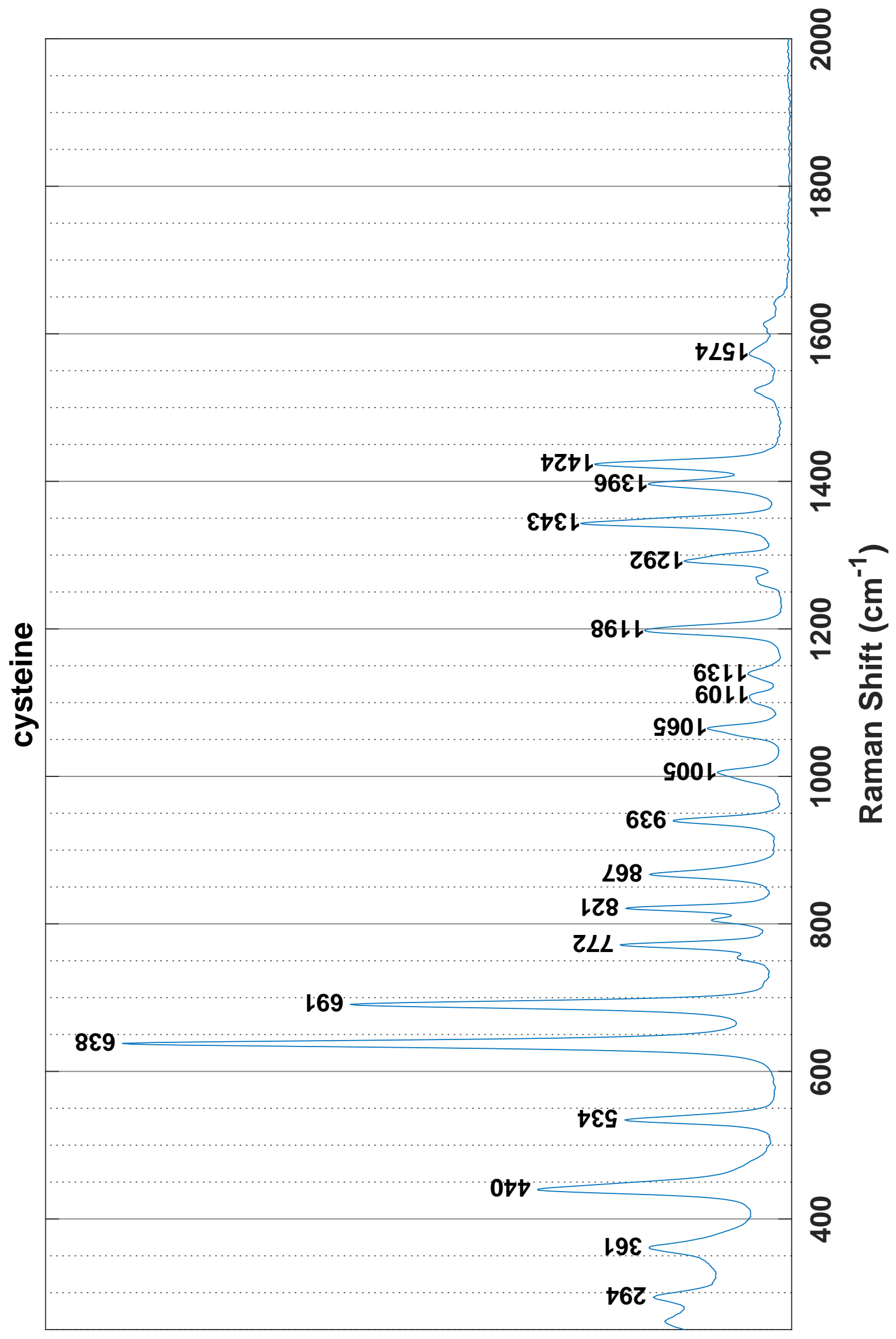




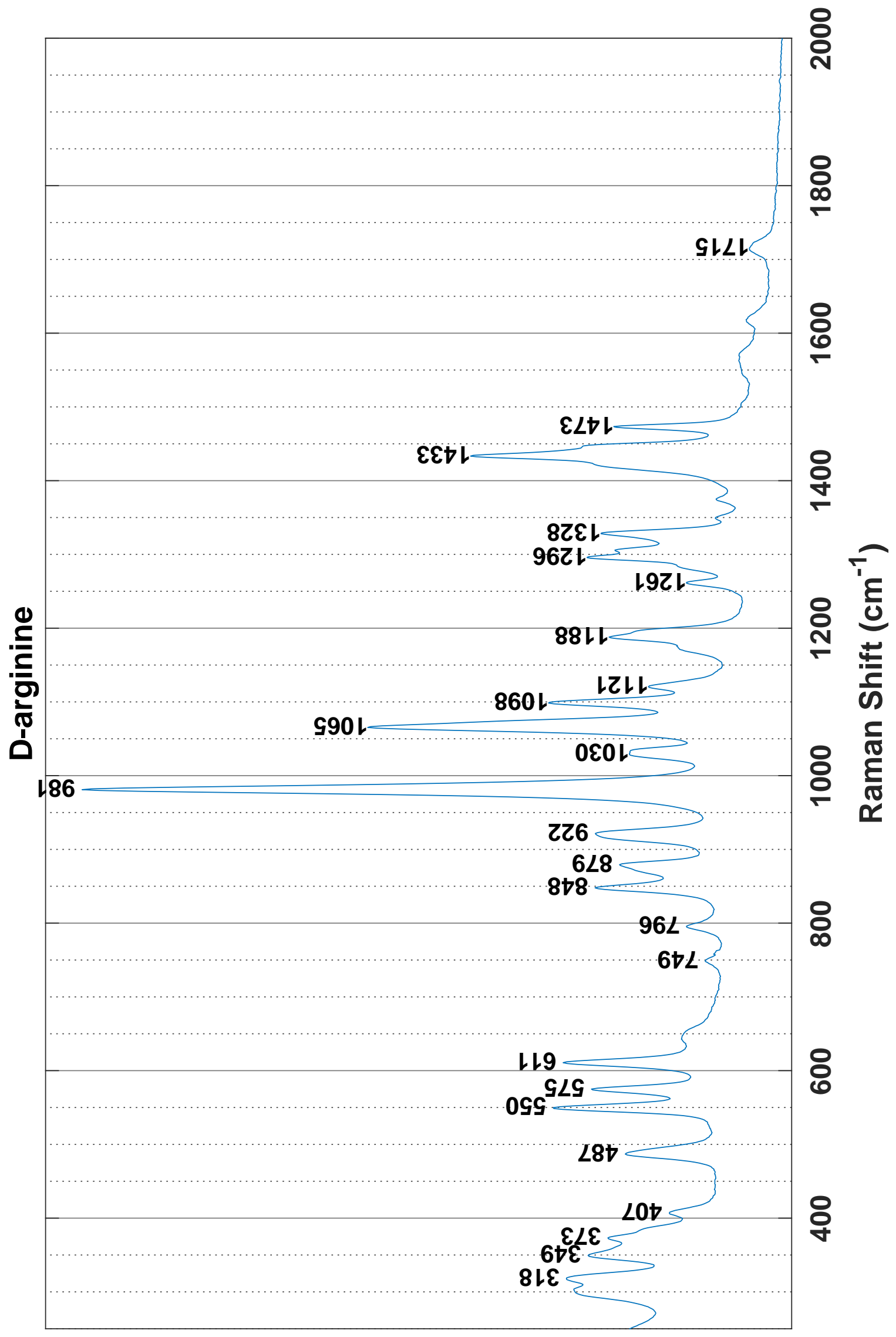




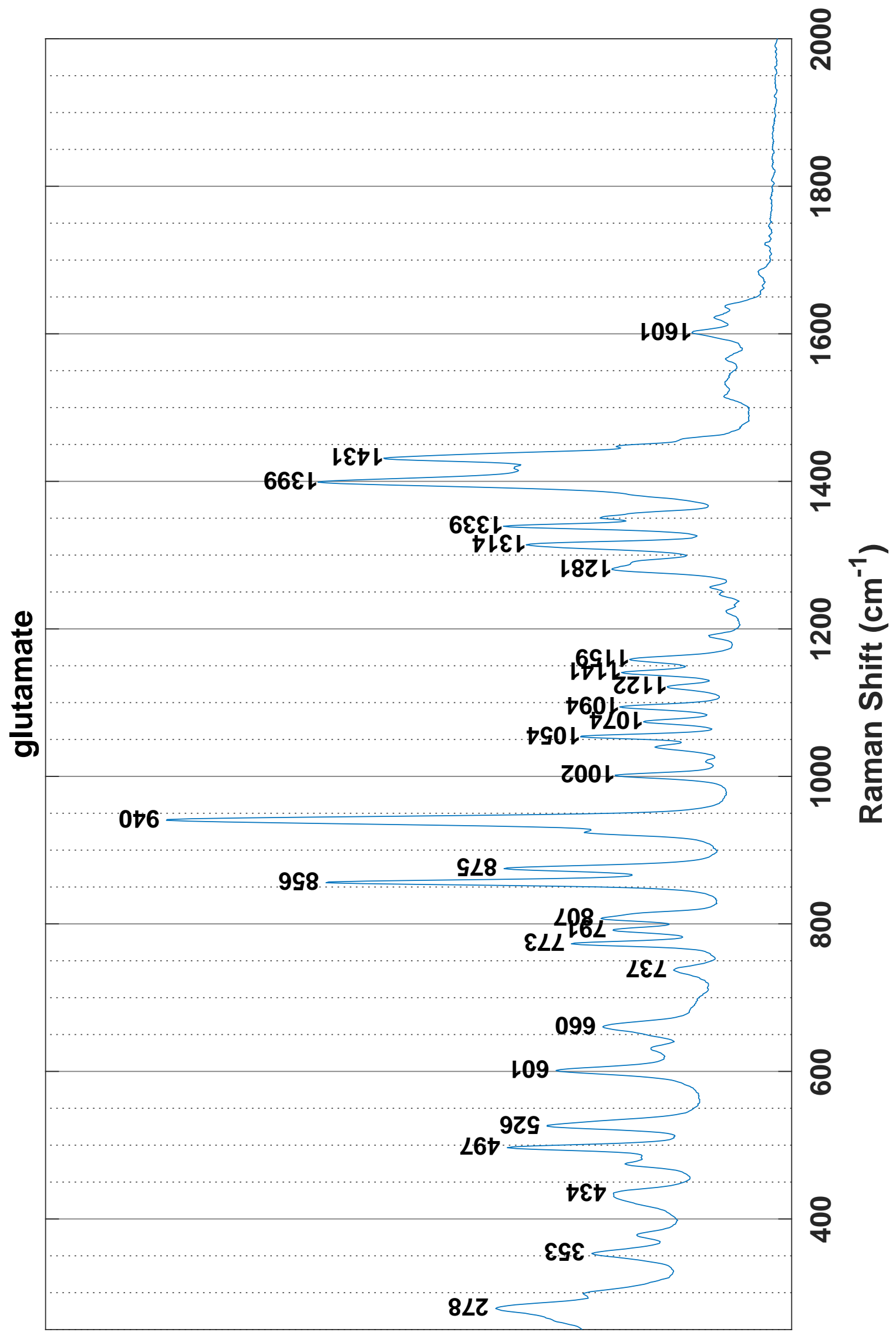




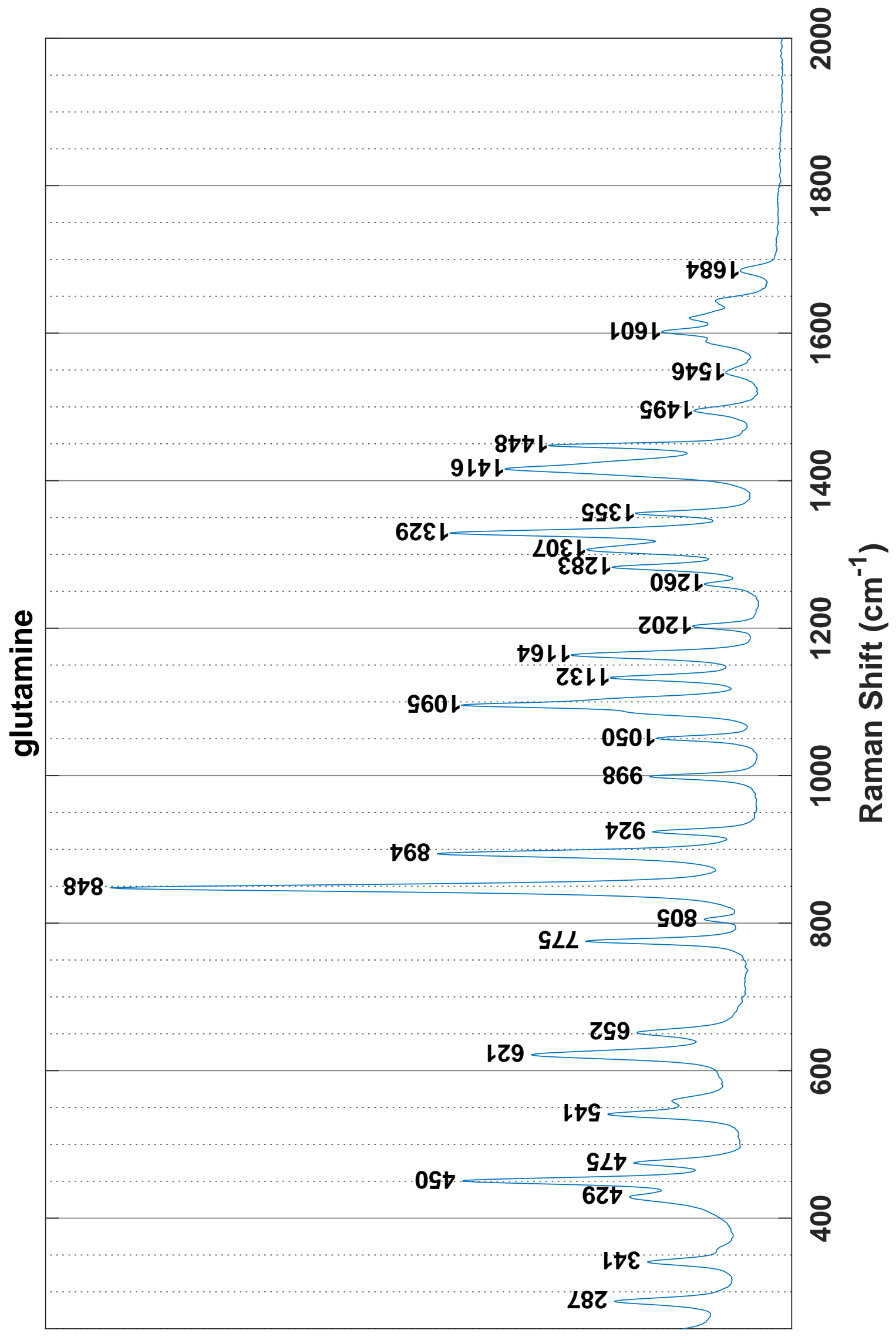




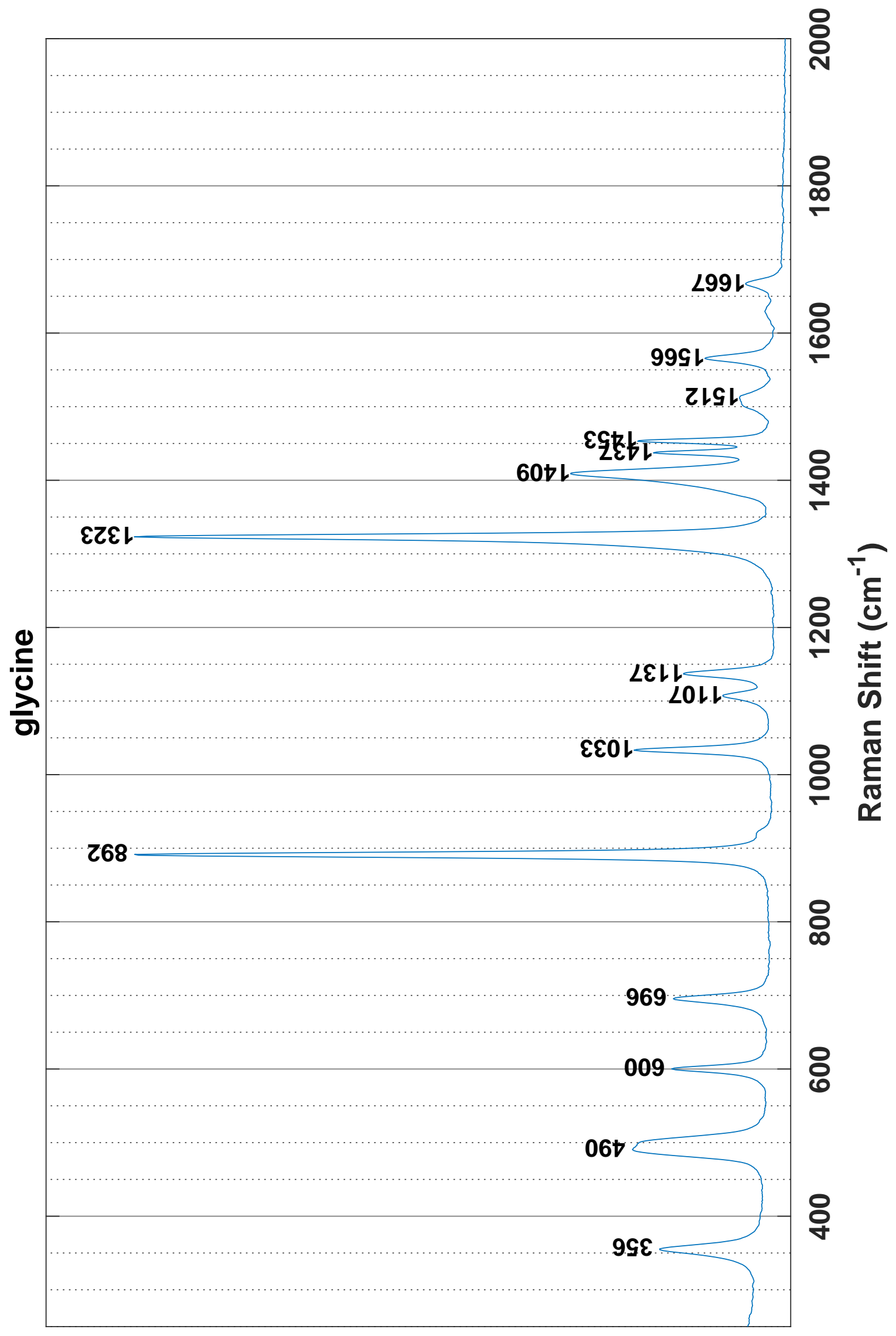




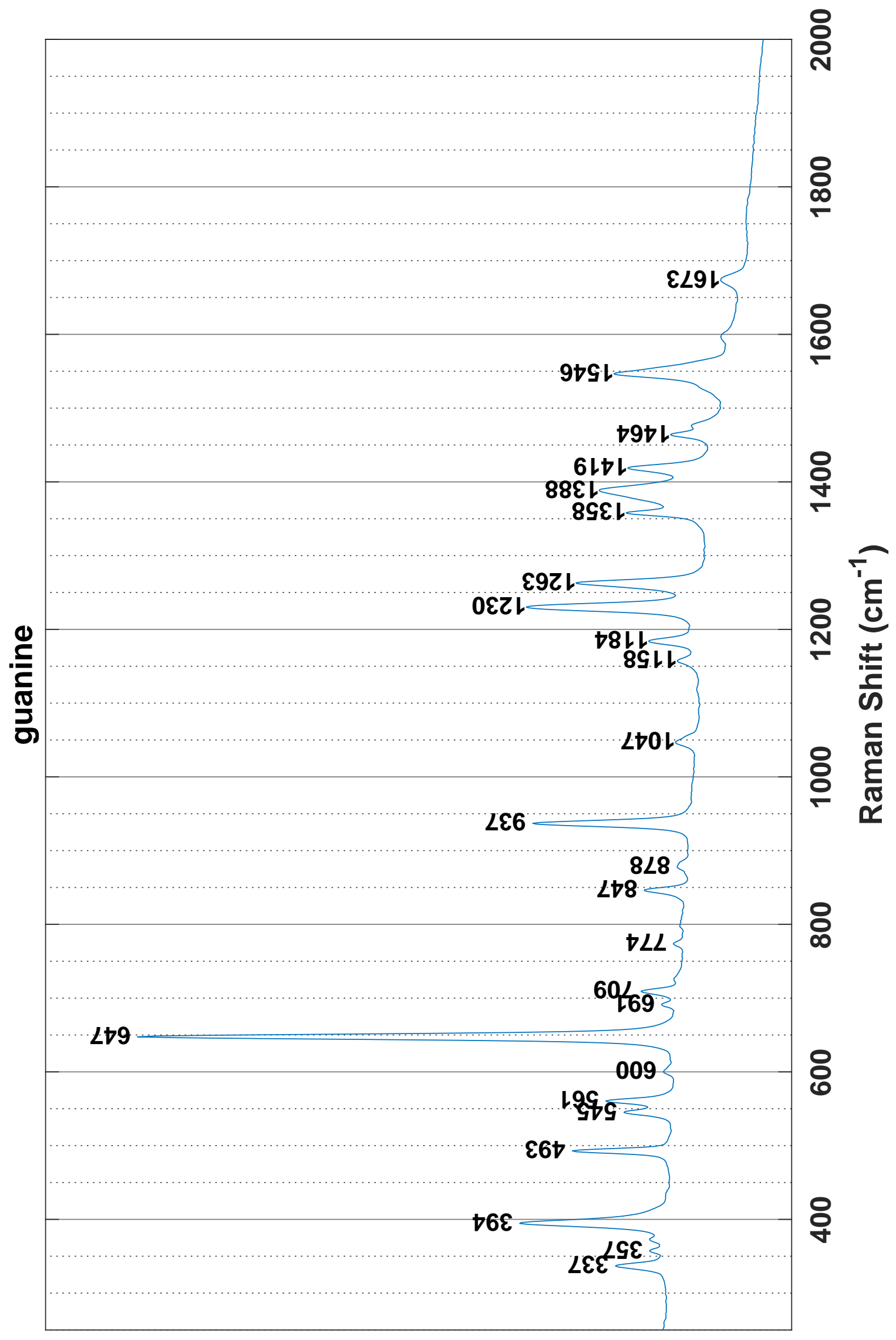




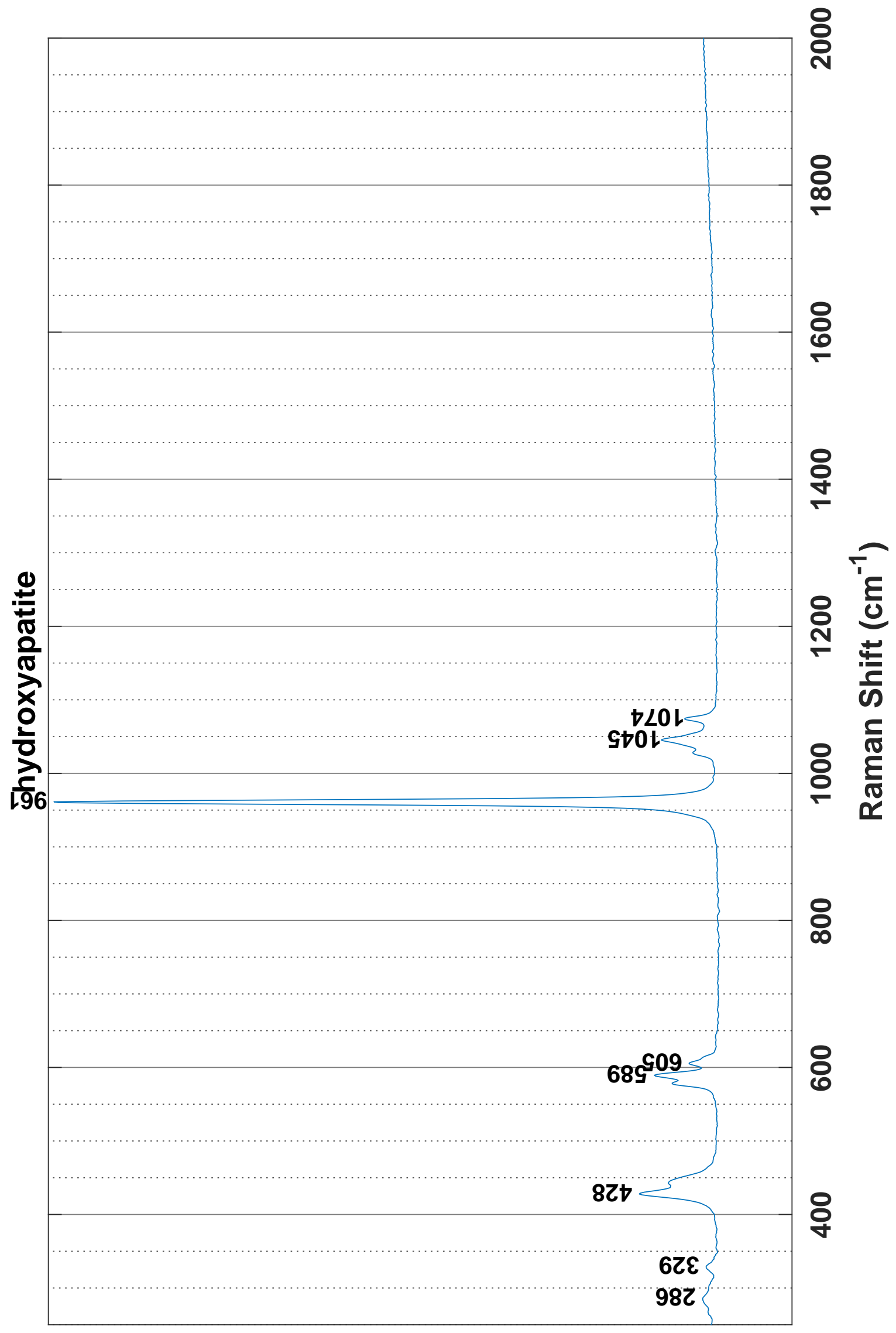




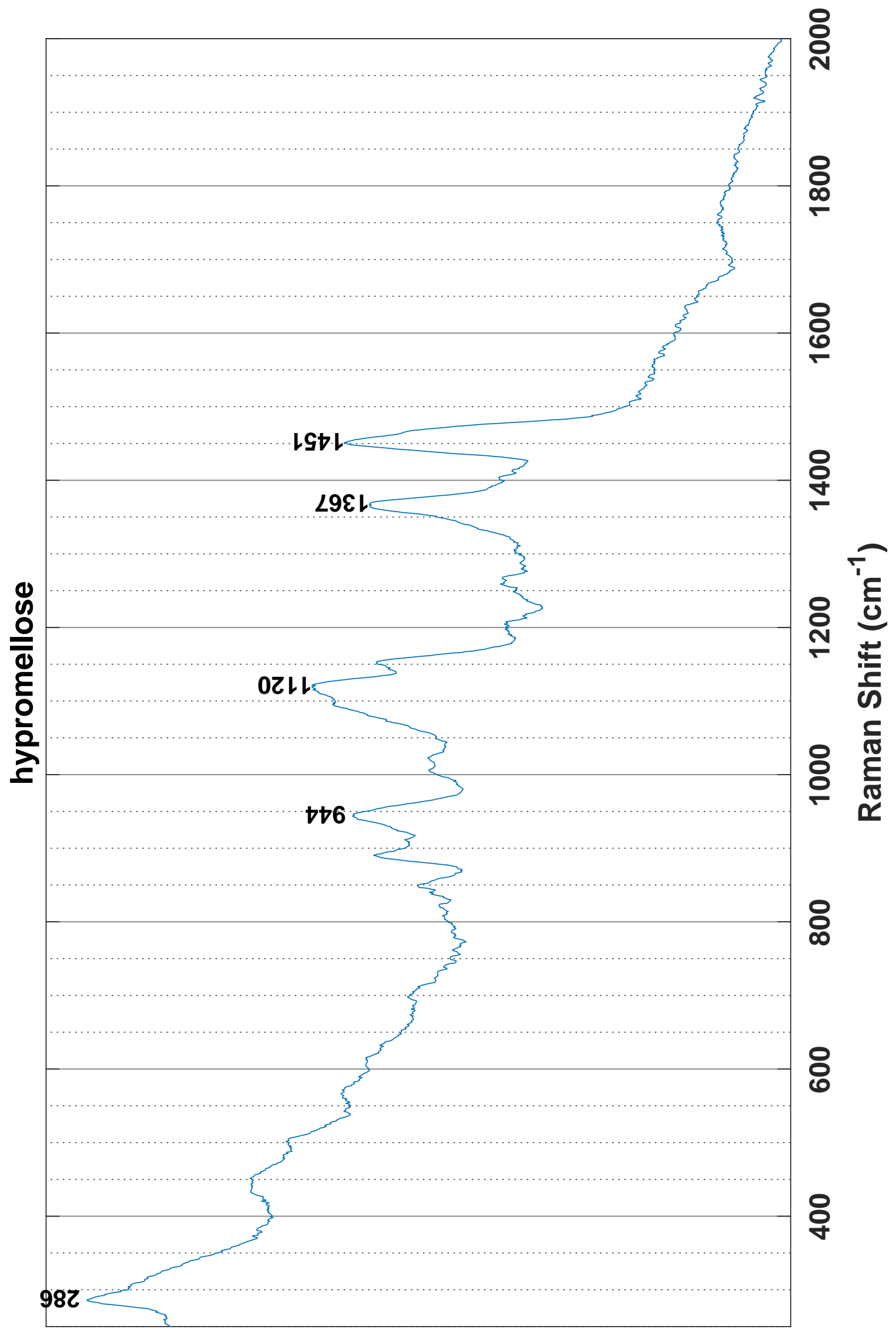




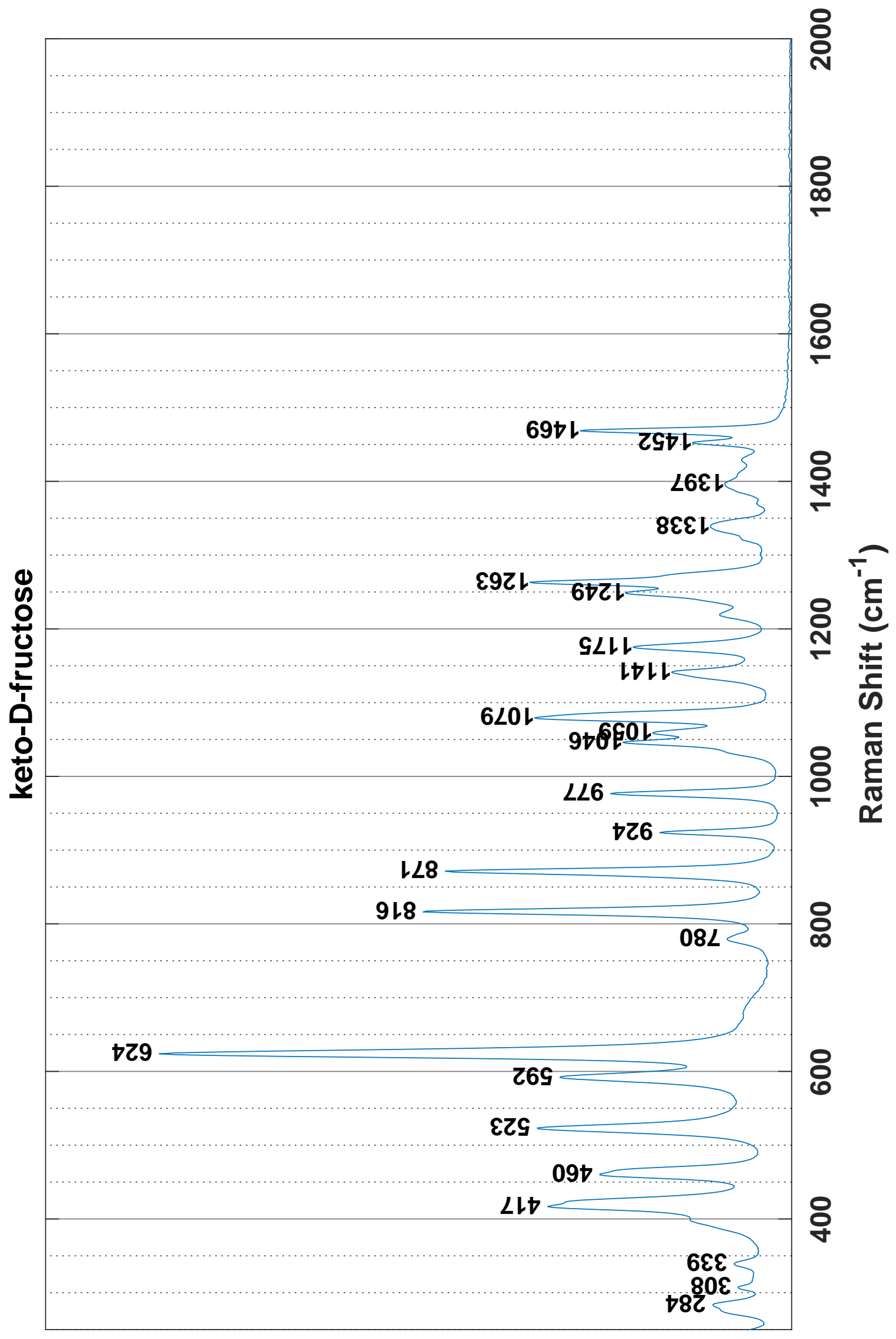




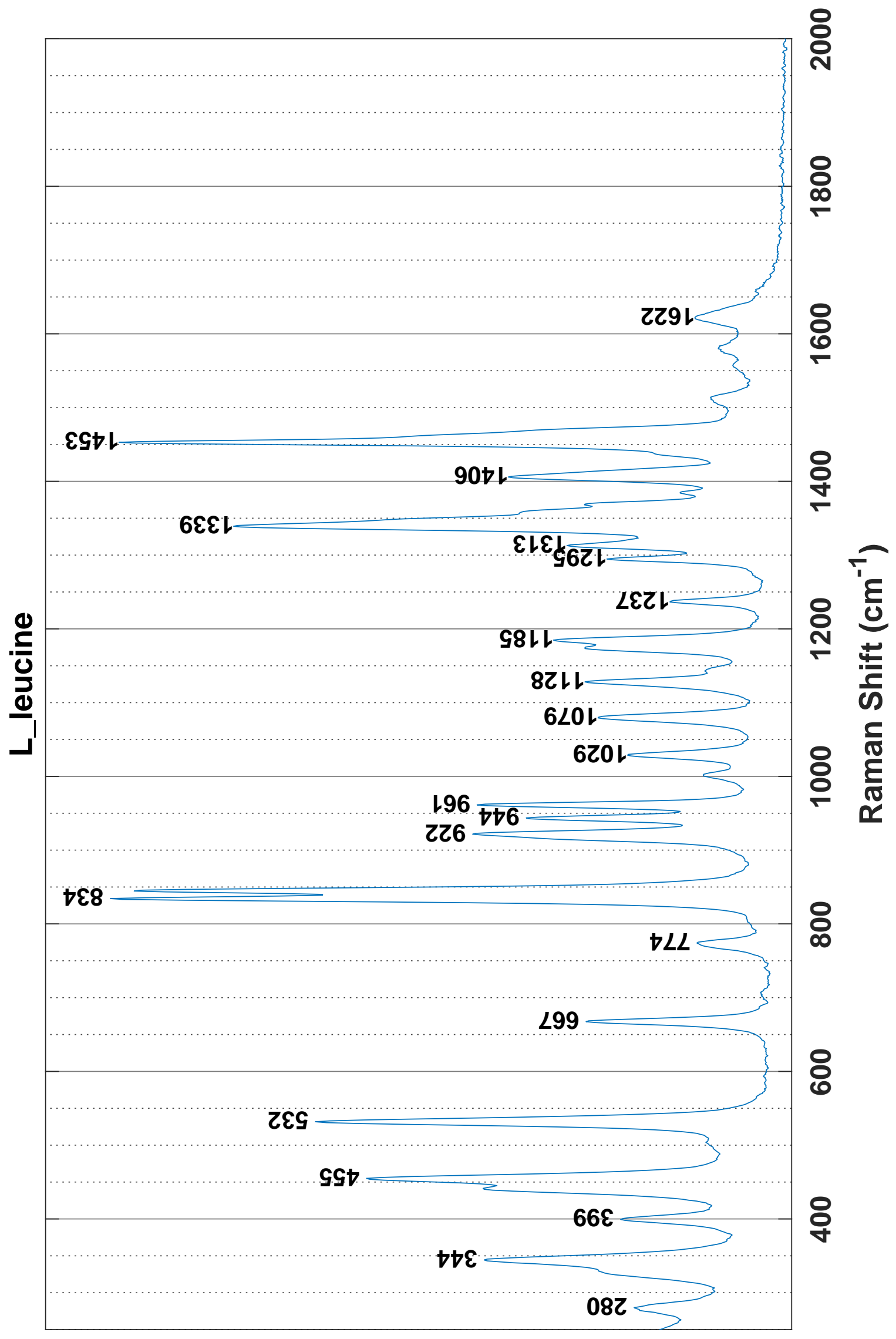




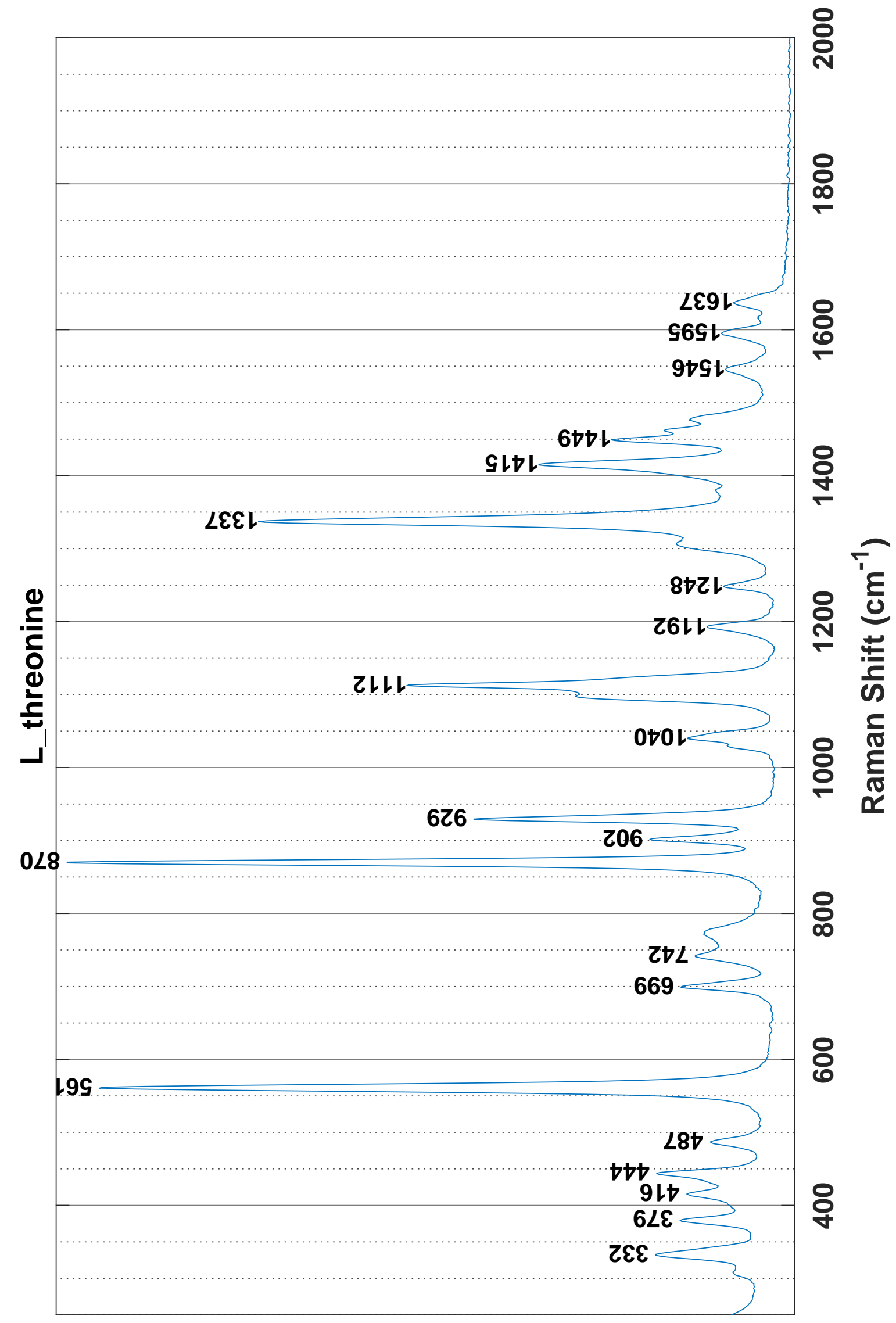




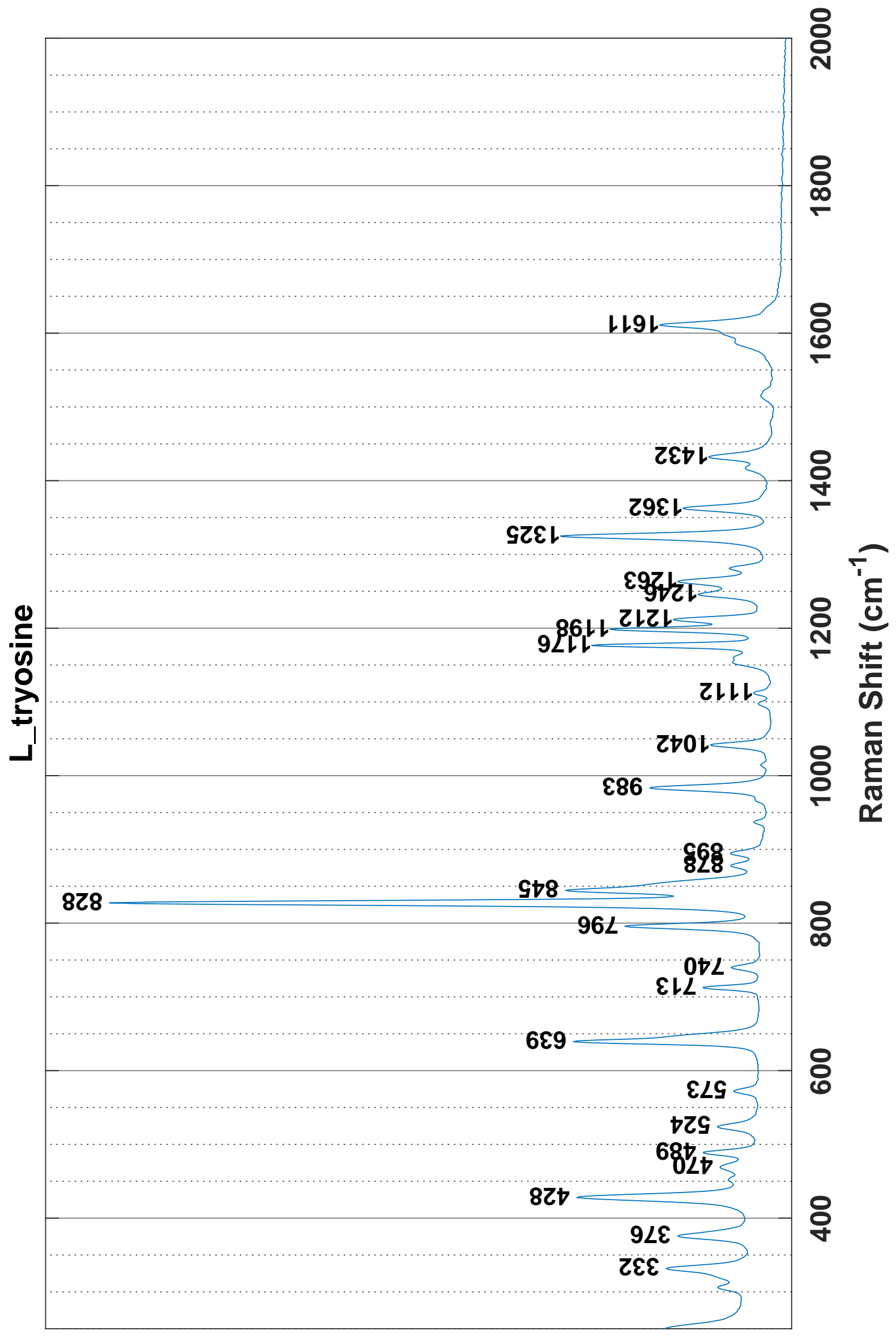




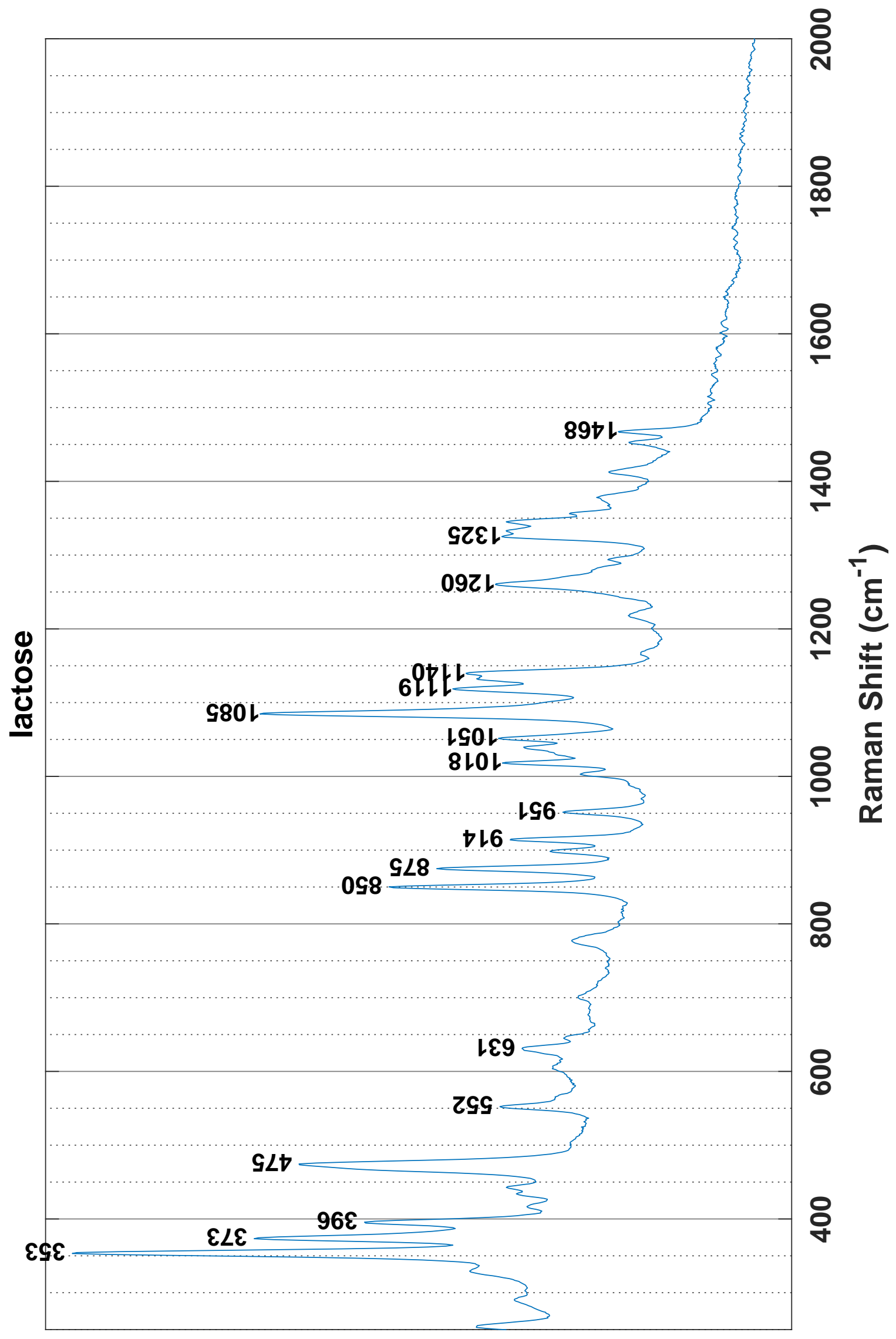




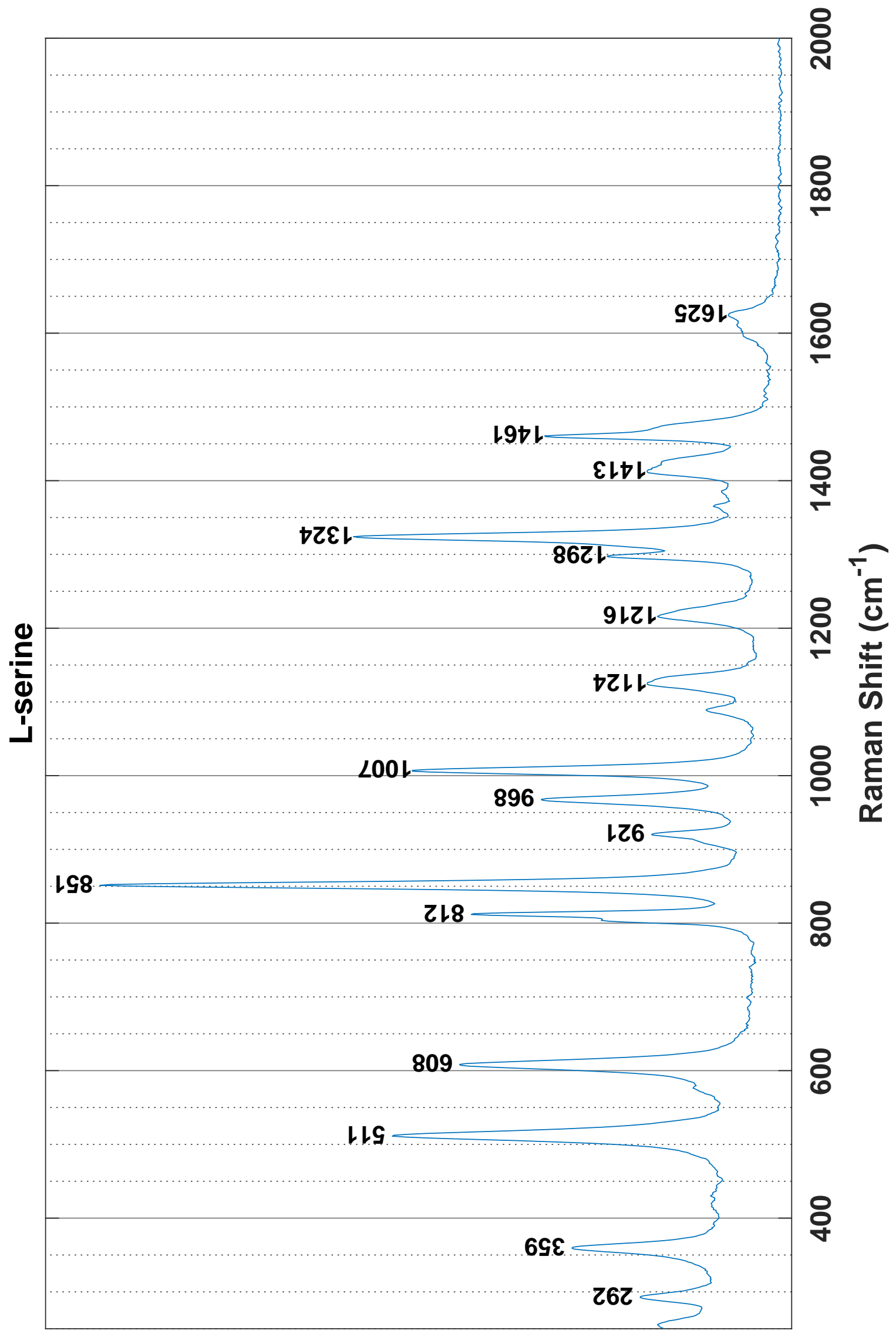




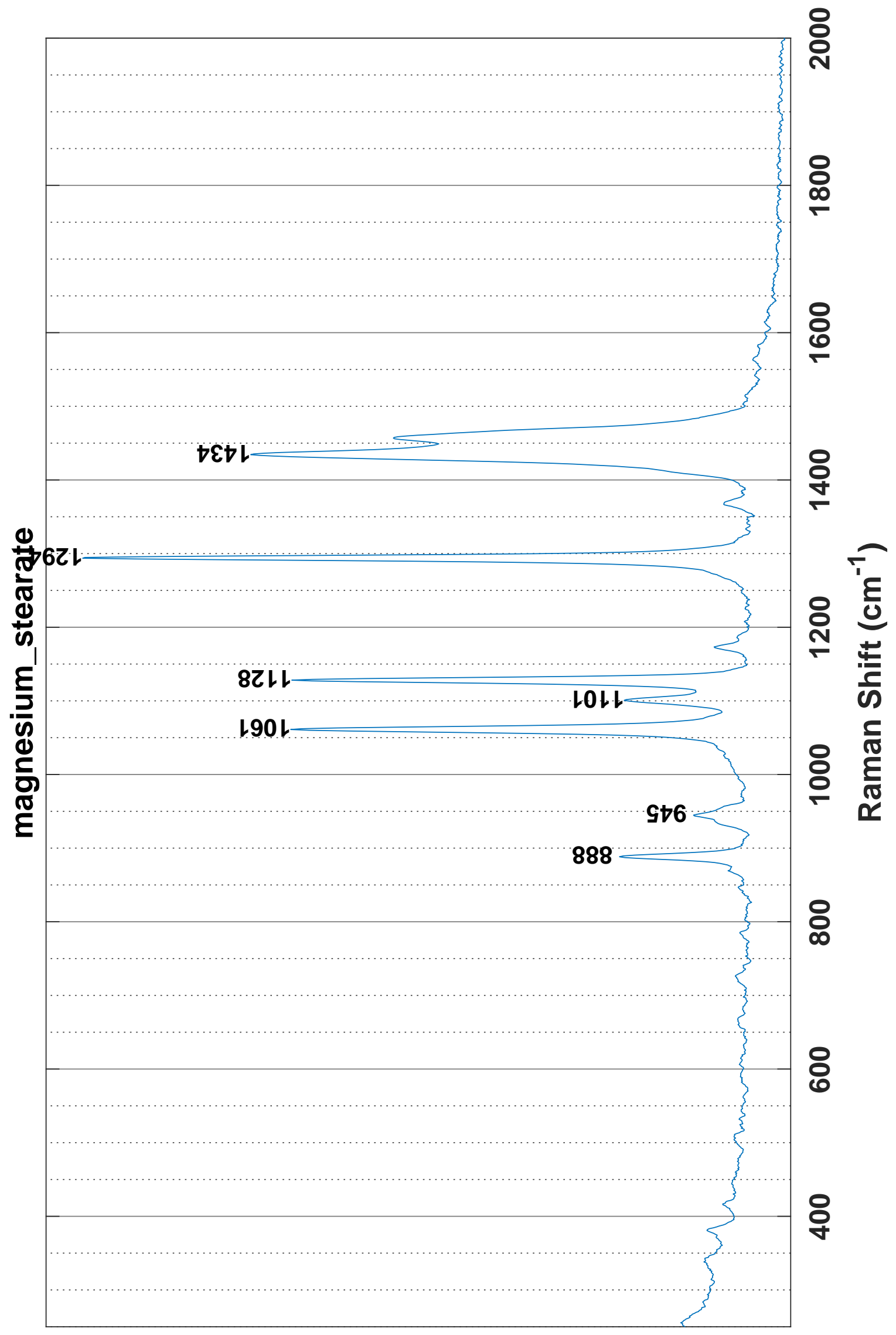




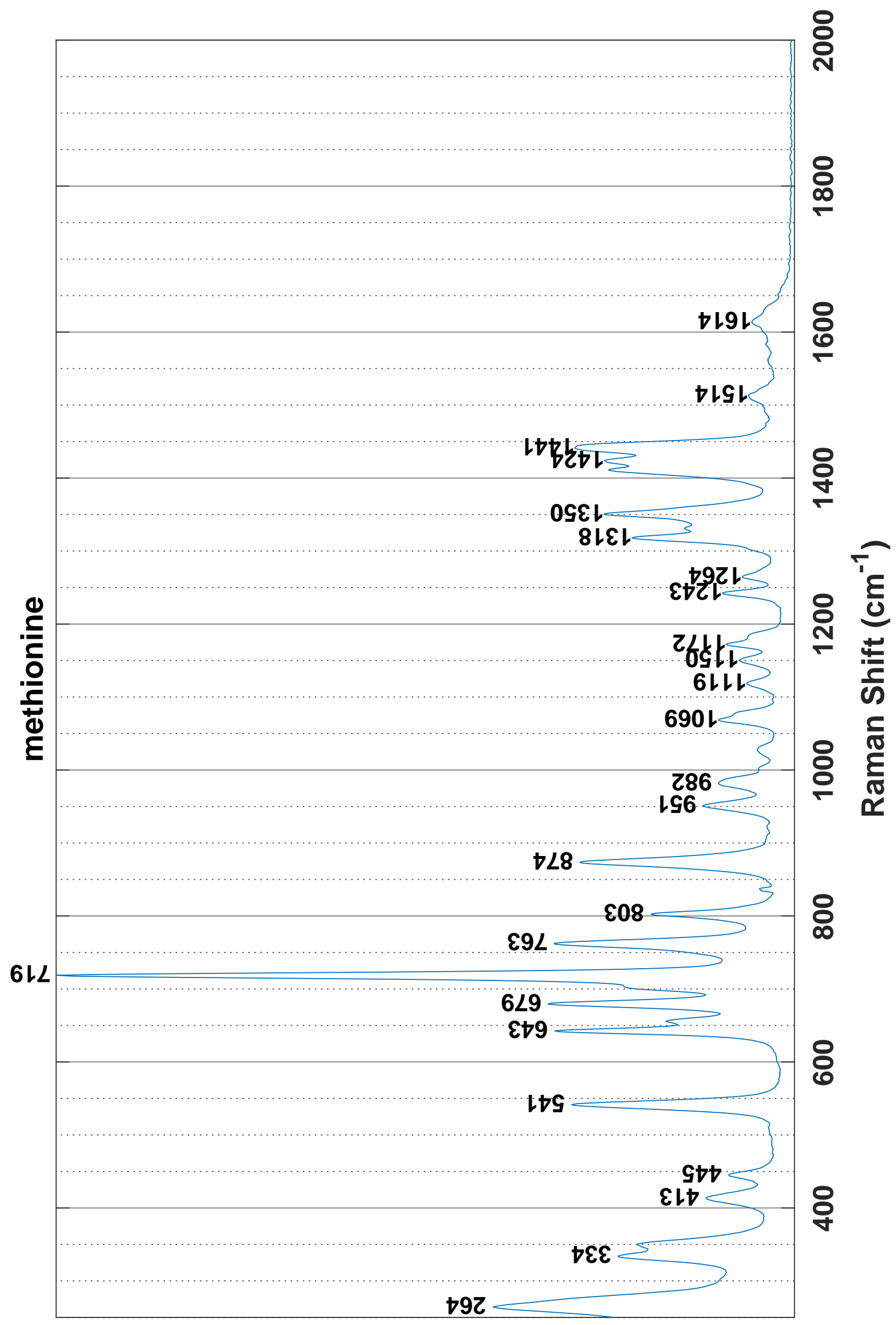




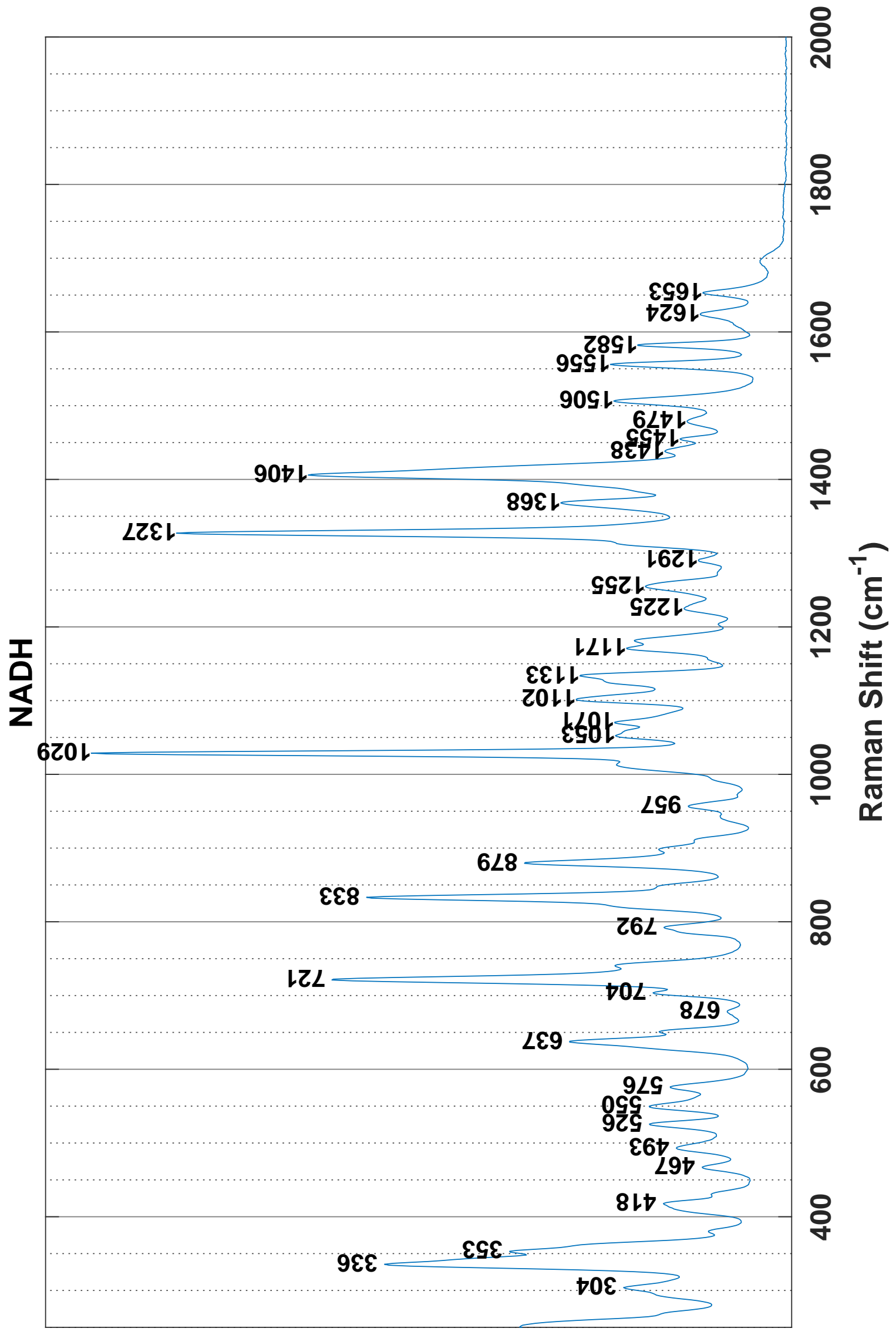




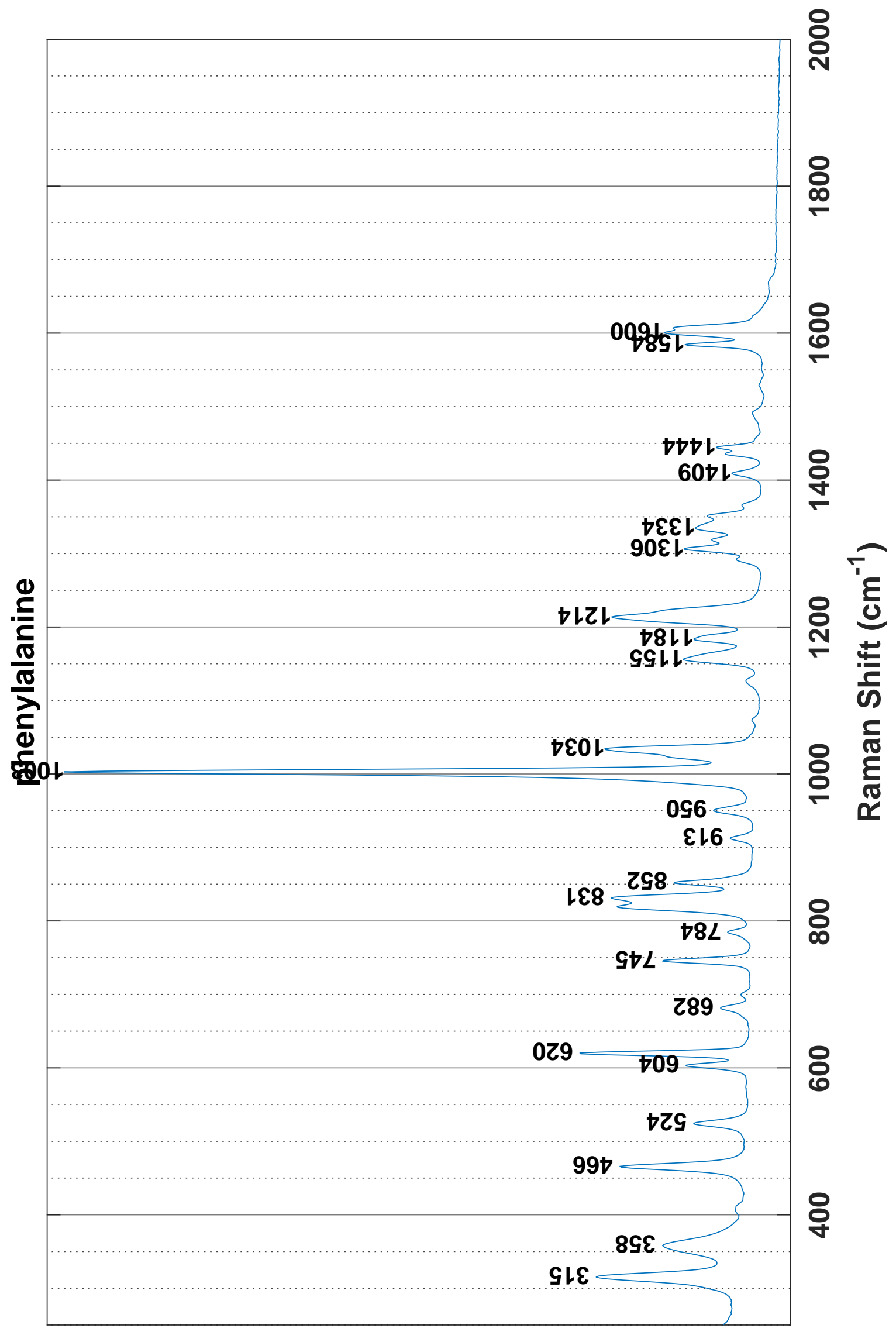




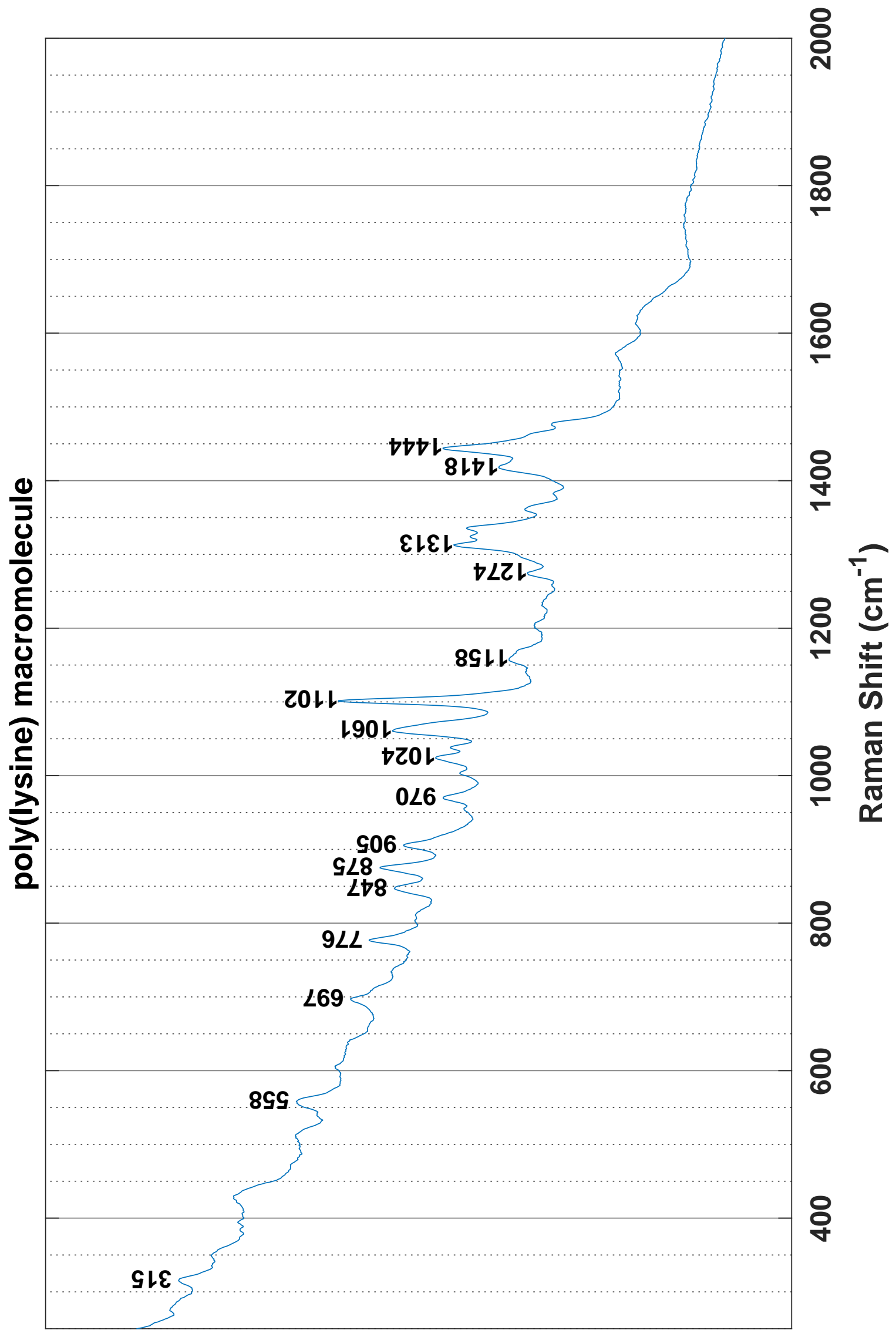




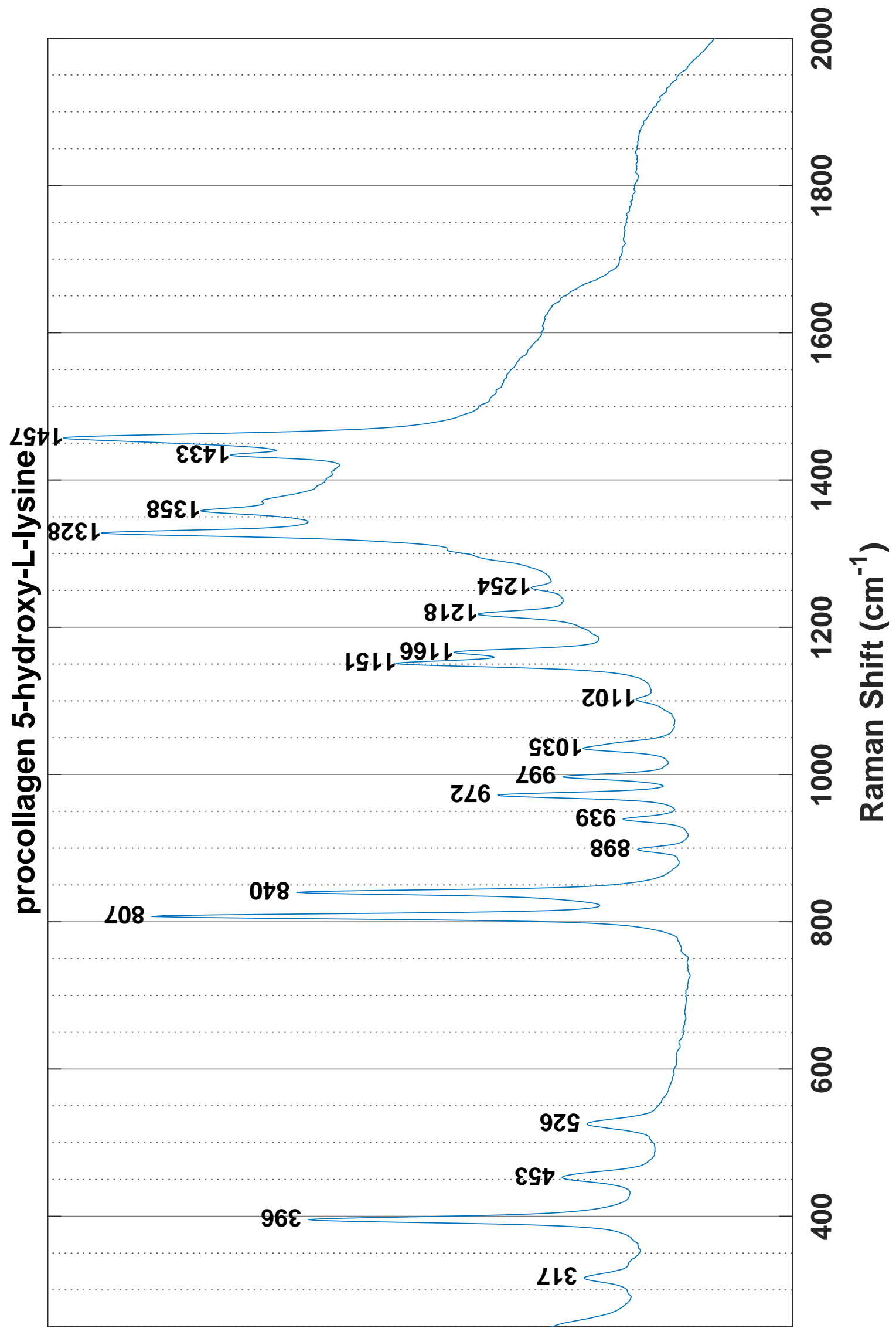




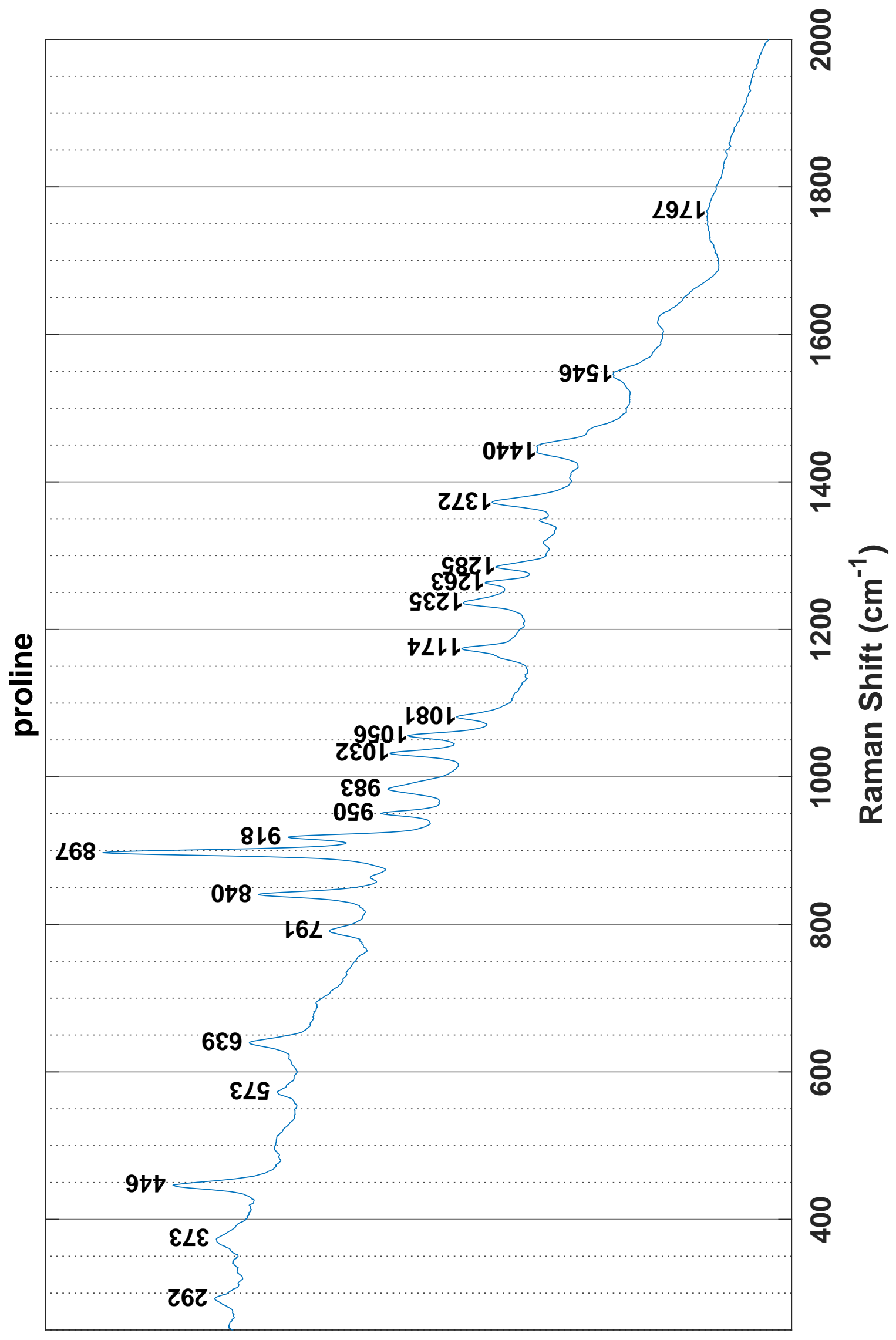




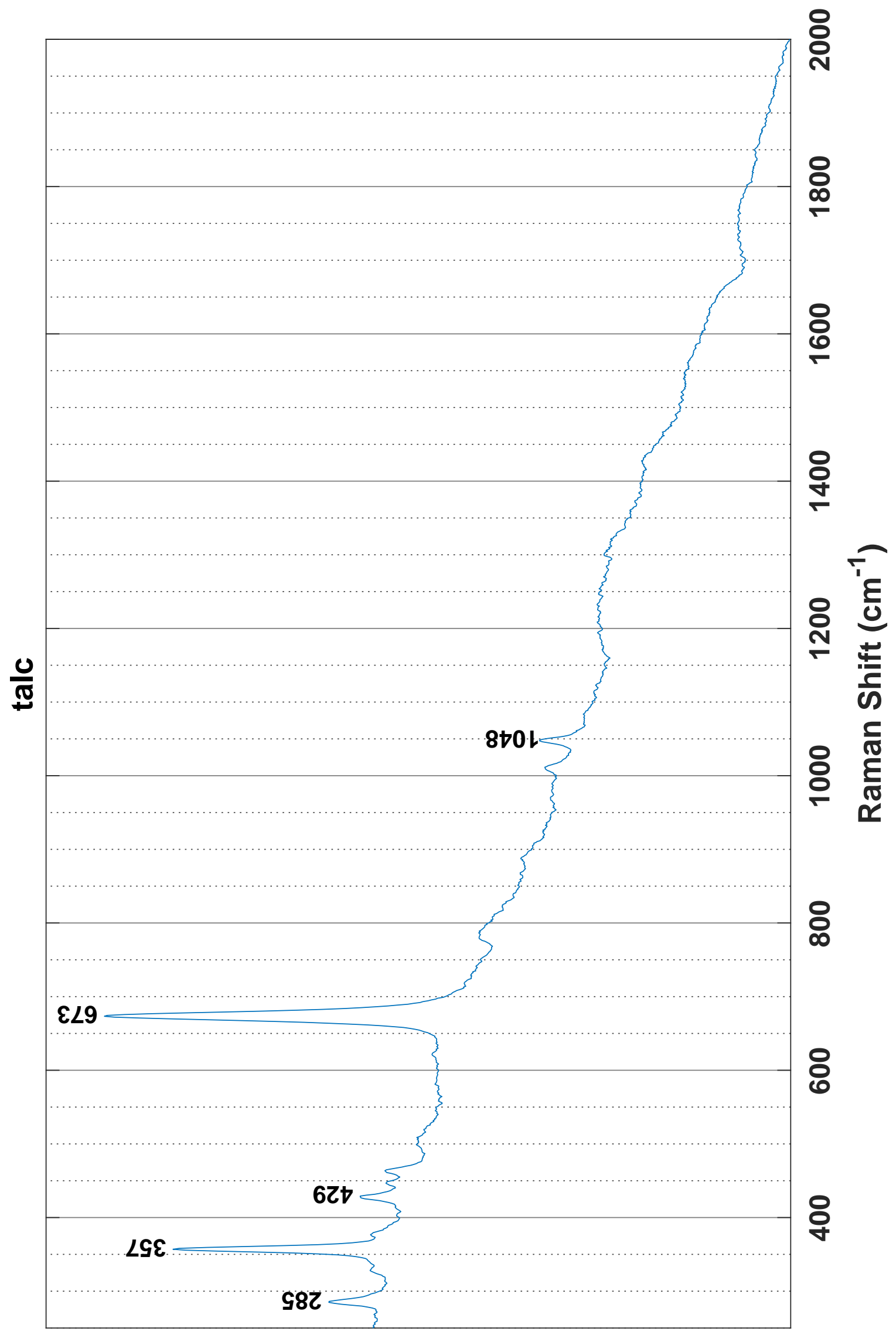




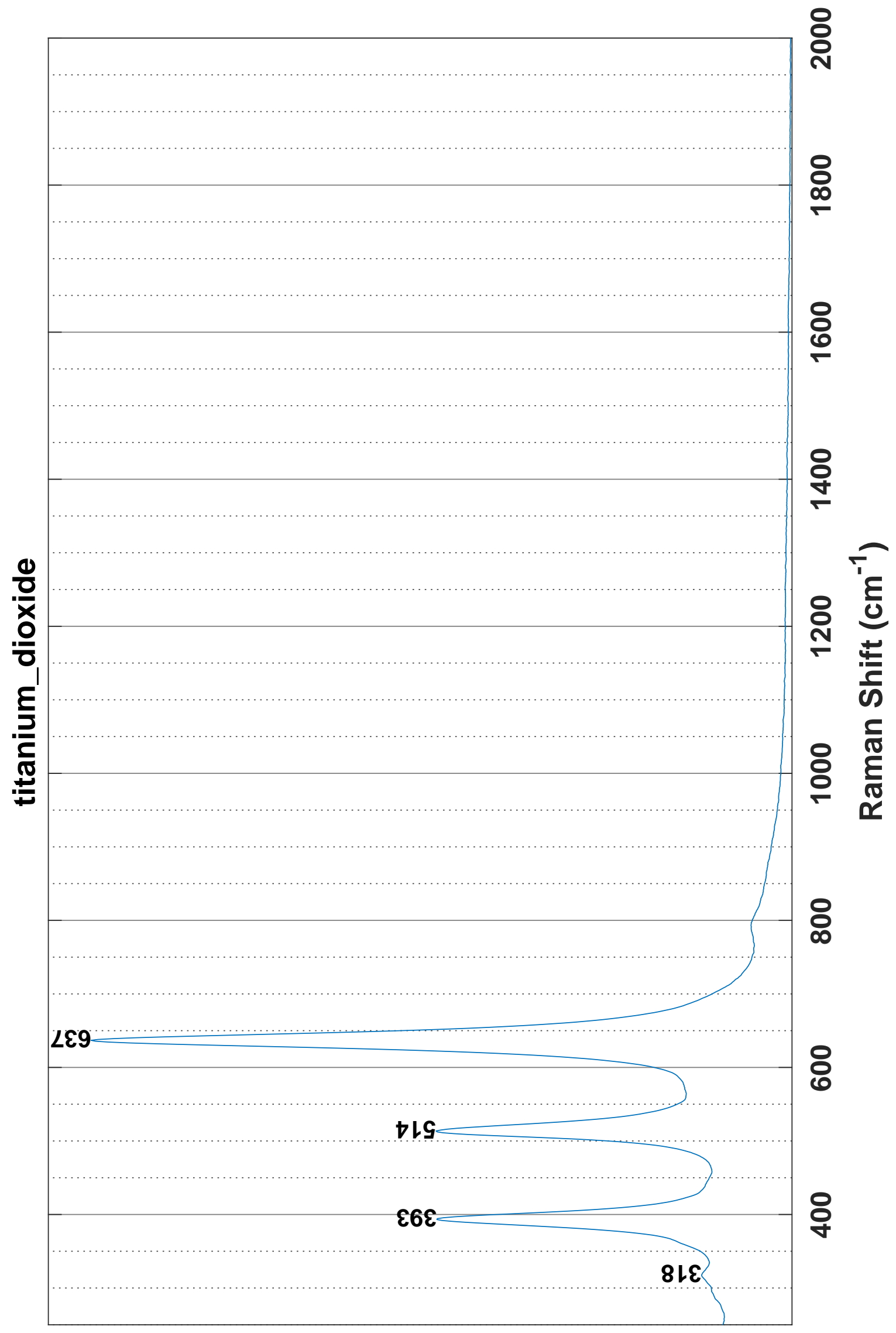




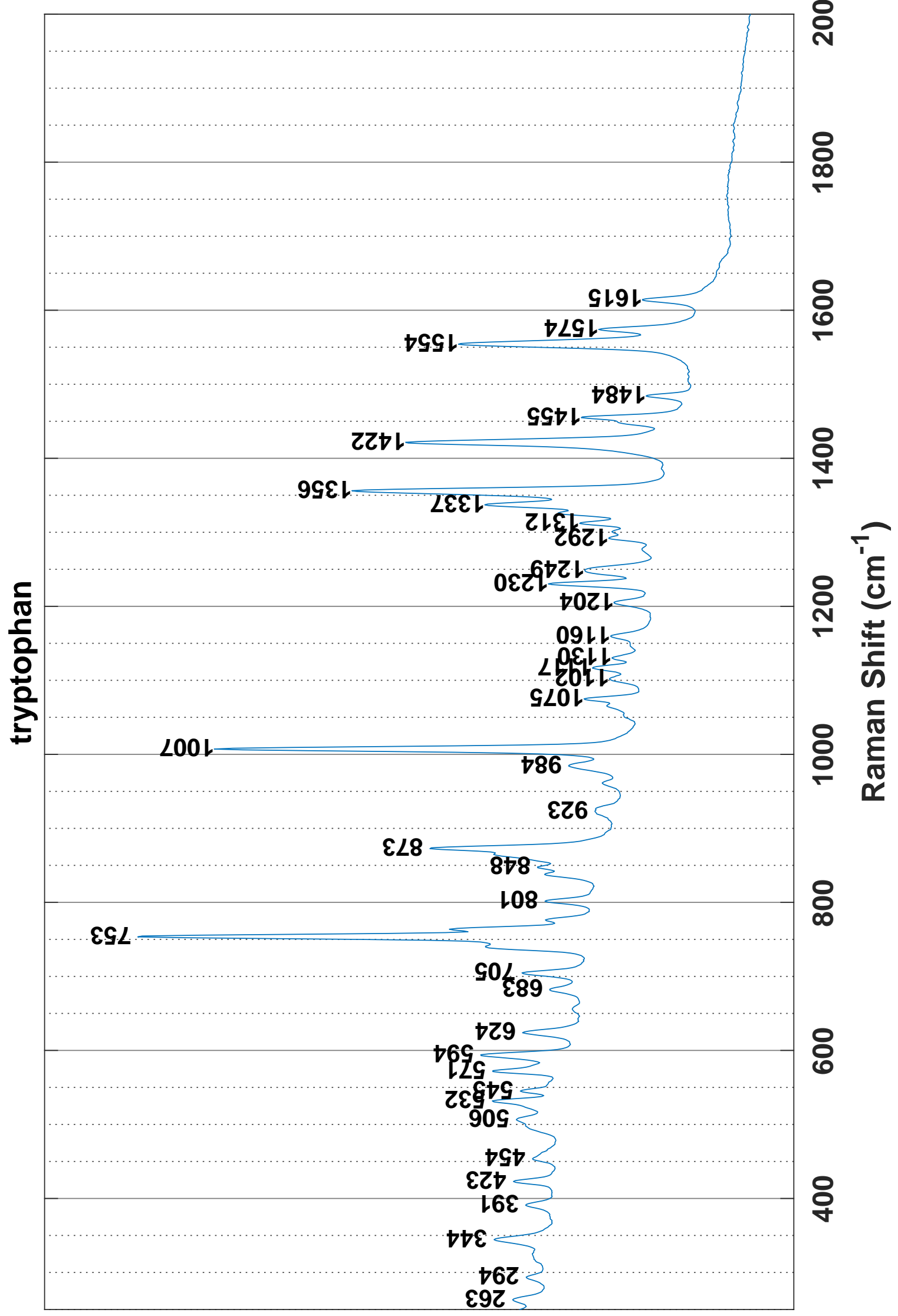




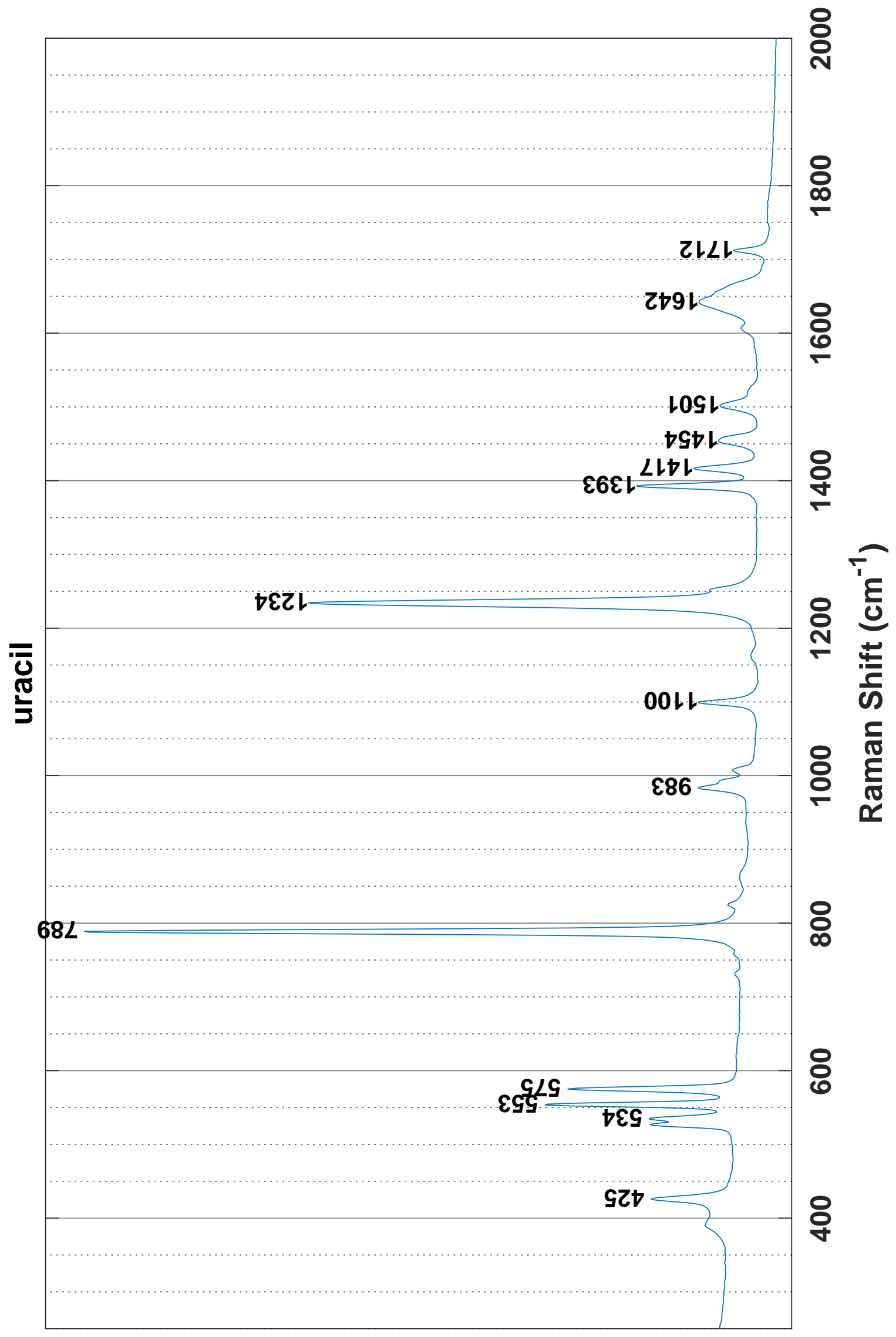




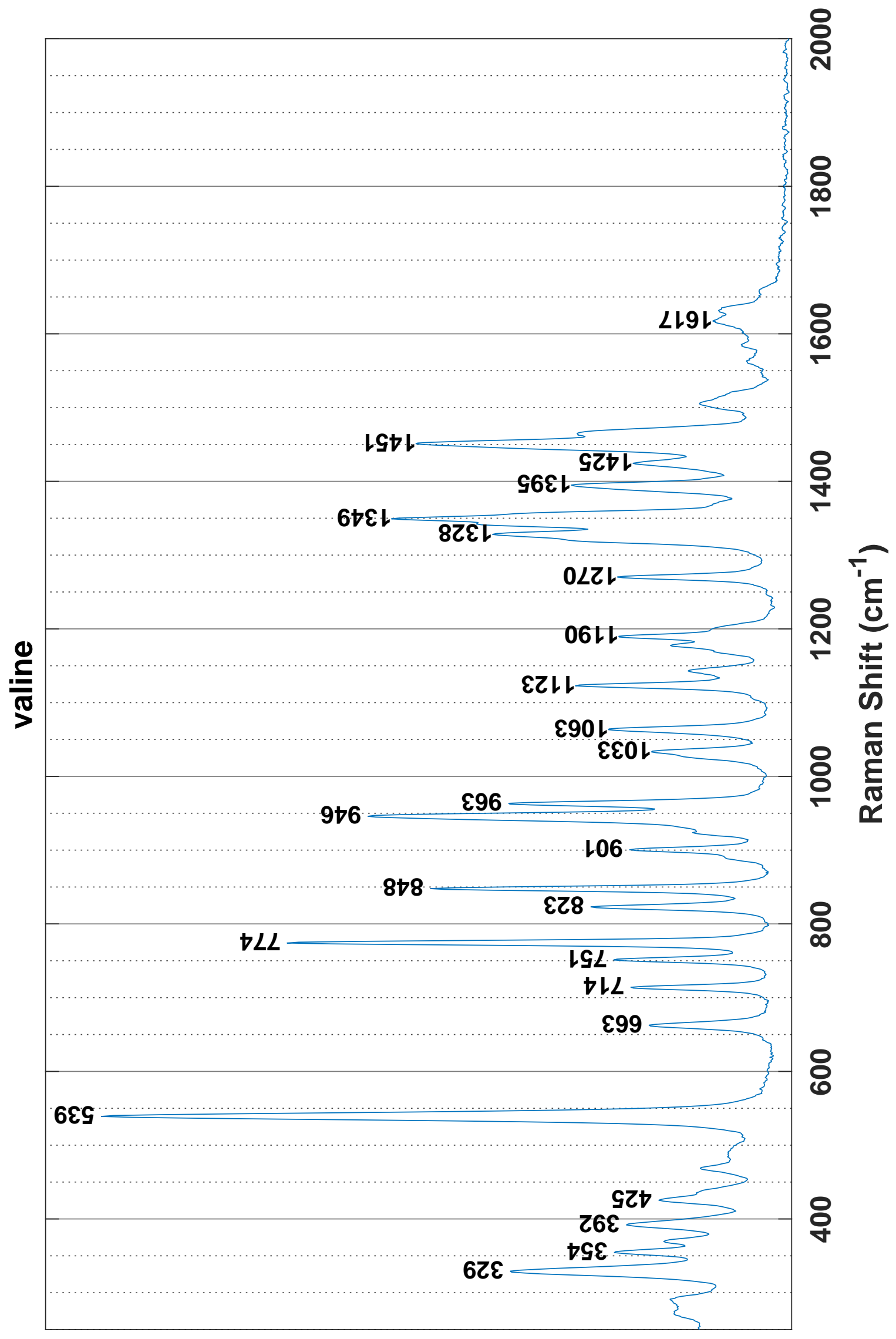




\section{References}

[1] A. Brolo, "Plasmonics for future biosensors," Nat. Photonics, vol. 6, no. November, pp. 709-713, 2012.

[2] S. Mousa, "Biosensors: the new wave in cancer diagnosis," Nanotechnol. Sci. Appl., p. 1, Dec. 2010.

[3] R. L. Rich and D. G. Myszka, "Survey of the 2009 commercial optical biosensor literature.," J. Mol. Recognit., vol. 24, no. 6, pp. 892-914, 2011.

[4] M. L. James and S. S. Gambhir, "A molecular imaging primer: modalities, imaging agents, and applications.," Physiol. Rev., vol. 92, no. 2, pp. 897-965, Apr. 2012.

[5] Y. Takeda and Y. Kajikawa, "Optics: a bibliometric approach to detect emerging research domains and intellectual bases," Scientometrics, vol. 78, no. 3, pp. 543-558, Oct. 2008.

[6] C. Kendall et al., "Vibrational spectroscopy: a clinical tool for cancer diagnostics.," Analyst, vol. 134, no. 6, pp. 1029-45, Jun. 2009.

[7] K. C. Bantz et al., "Recent progress in SERS biosensing.," Phys. Chem. Chem. Phys., vol. 13, no. 24, pp. 11551-67, Jun. 2011.

[8] S. G. Demos, A. J. Vogel, and A. H. Gandjbakhche, "Advances in optical spectroscopy and imaging of breast lesions.," J. Mammary Gland Biol. Neoplasia, vol. 11, no. 2, pp. 165-81, Apr. 2006.

[9] J. R. Lombardi, M. Leona, and T. Vo-dinh, "Report Title: Development of Advanced Raman Spectroscopy Methods and Databases For The Evaluation of Trace Evidence and The Examination of Questioned Documents ( Phase I) Authors : John R . Lombardi , Center for Analysis of Structures and Interfaces , C,” 2009.

[10] J. a Dougan and K. Faulds, "Surface enhanced Raman scattering for multiplexed detection.," Analyst, vol. 137, no. 3, pp. 545-54, Feb. 2012.

[11] K. Kneipp et al., "Single Molecule Detection Using Surface-Enhanced Raman Scattering 
(SERS)," Physical Review Letters, vol. 78. pp. 1667-1670, 1997.

[12] K. Kneipp, H. Kneipp, G. Deinum, I. Itzkan, R. R. Dasari, and M. S. Feld, "SingleMolecule Detection of a Cyanine Dye in Silver Colloidal Solution Using Near-Infrared Surface-Enhanced Raman Scattering,” Applied Spectroscopy, vol. 52. pp. 175-178, 1998.

[13] J. Zhao, H. Lui, D. I. McLean, and H. Zeng, "Integrated real-time Raman system for clinical in vivo skin analysis.," Skin Res. Technol., vol. 14, no. 4, pp. 484-92, Nov. 2008.

[14] S. Duraipandian, "Real-time Raman spectroscopy for in vivo, online gastric cancer diagnosis during clinical endoscopic examination."

[15] H. Wang, A. M. D. Lee, H. Lui, D. I. McLean, and H. Zeng, "A method for accurate in vivo micro-Raman spectroscopic measurements under guidance of advanced microscopy imaging.," Sci. Rep., vol. 3, no. 1, p. 1890, Jan. 2013.

[16] K. Kong, C. Kendall, N. Stone, and I. Notingher, "Raman spectroscopy for medical diagnostics - From in-vitro biofluid assays to in-vivo cancer detection," Advanced Drug Delivery Reviews, vol. 89. pp. 121-134, 2015.

[17] M. Jermyn et al., "A review of Raman spectroscopy advances with an emphasis on clinical translation challenges in oncology," Phys. Med. Biol., vol. 61, no. 23. pp. R370R400, 2016.

[18] Q. Tu and C. Chang, "Diagnostic applications of Raman spectroscopy.," Nanomedicine, vol. 8, no. 5, pp. 545-58, Jul. 2012.

[19] E. Hanlon and R. Manoharan, "Prospects for in vivo Raman spectroscopy," Phys. Med. Biol., vol. 1, 2000.

[20] T. J. Moritz, D. S. Taylor, D. M. Krol, J. Fritch, and J. W. Chan, "Detection of doxorubicin-induced apoptosis of leukemic T-lymphocytes by laser tweezers Raman spectroscopy.," Biomed. Opt. Express, vol. 1, no. 4, pp. 1138-1147, Jan. 2010.

[21] H. Abramczyk, B. Brozek-Pluska, J. Surmacki, J. Jablonska-Gajewicz, and R. Kordek, 
"Raman 'optical biopsy' of human breast cancer.," Prog. Biophys. Mol. Biol., vol. 108, no. 1-2, pp. 74-81, Jan. 2012.

[22] Y. S. Huh, A. J. Chung, and D. Erickson, "Surface enhanced Raman spectroscopy and its application to molecular and cellular analysis," Microfluid. Nanofluidics, vol. 6, no. 3, pp. 285-297, Jan. 2009.

[23] D. Cialla et al., "Surface-enhanced Raman spectroscopy (SERS): progress and trends.," Anal. Bioanal. Chem., vol. 403, no. 1, pp. 27-54, Apr. 2012.

[24] Nobel Prize Organization, “The Nobel Prize for Physics in 1930,” 2018. [Online]. Available: https://www.nobelprize.org/prizes/physics/1930/summary/. [Accessed: 30-Sep$2018]$.

[25] G. Keresztury, "Raman Spectroscopy: Theory," in Handbook of Vibrational Spectroscopy, J. M. Chalmers and P. R. Griffiths, Eds. J. Wiley, 2002.

[26] M. Fleischmann, P. J. Hendra, and A. J. McQuillan, "Raman spectra of pyridine adsorbed at a silver electrode," Chem. Phys. Lett., vol. 26, no. 2, pp. 163-166, 1974.

[27] A. Otto, "Surface enhanced Raman scattering (SERS), what do we know?," Appl. Surf. Sci., vol. 6, no. 3, pp. 309-355, 1980.

[28] J. Gersten and A. Nitzan, "Electromagnetic theory of enhanced Raman scattering by molecules adsorbed on rough surfaces," J. Chem. Phys., vol. 73, no. 7, pp. 3023-3037, 1980.

[29] H. Metiu and P. Das, "The Electromagnetic Theory of Surface Enhanced Spectroscopy," Annu. Rev. Phys. Chem., vol. 35, no. 1, pp. 507-536, 1984.

[30] E. Hao and G. C. Schatz, "Electromagnetic fields around silver nanoparticles and dimers.," J. Chem. Phys., vol. 120, no. 1, pp. 357-66, Jan. 2004.

[31] K. a Willets and R. P. Van Duyne, "Localized surface plasmon resonance spectroscopy and sensing.," Аnnu. Rev. Phys. Chem., vol. 58, no. October, pp. 267-97, Jan. 2007. 
[32] G. C. Schatz and R. P. Van Duyne, "Electromagnetic Mechanism of Surface-Enhanced Spectroscopy," in Handbook of Vibrational Spectroscopy, 2006.

[33] J. M. Chalmers and P. R. Griffiths, "Handbook of vibrational spectroscopy," no. v. 1, J. Wiley, 2002.

[34] A. Wokaun, "Surface-Enhanced Electromagnetic Processes," Solid State Phys. - Adv. Res. Appl., vol. 38, no. C, pp. 223-294, 1984.

[35] A. B. Zrimsek et al., "Single-Molecule Chemistry with Surface- and Tip-Enhanced Raman Spectroscopy," Chemical Reviews, vol. 117, no. 11. pp. 7583-7613, 2017.

[36] S.-Y. Ding, E.-M. You, Z.-Q. Tian, and M. Moskovits, "Electromagnetic theories of surface-enhanced Raman spectroscopy," Chem. Soc. Rev., vol. 46, no. 13, pp. 4042-4076, 2017.

[37] K. Kneipp, "Chemical Contribution to SERS Enhancement: An Experimental Study on a Series of Polymethine Dyes on Silver Nanoaggregates," J. Phys. Chem. C, vol. 120, no. 37, pp. 21076-21081, 2016.

[38] W.-H. Park and Z. H. Kim, "Charge Transfer Enhancement in the SERS of a Single Molecule," Nano Lett., vol. 10, no. 10, pp. 4040-4048, 2010.

[39] J. R. Lombardi and R. L. Birke, "The theory of surface-enhanced Raman scattering," J. Chem. Phys., vol. 136, no. 14, p. 144704, 2012.

[40] J. R. Lombardi and R. L. Birke, "A unified approach to surface-enhanced raman spectroscopy," J. Phys. Chem. C, vol. 112, no. 14, pp. 5605-5617, 2008.

[41] J. R. Lombardi and R. L. Birke, "A unified view of surface-enhanced raman scattering," Acc. Chem. Res., vol. 42, no. 6, pp. 734-742, 2009.

[42] M. D. Keller, E. M. Kanter, and A. Mahadevan-Jansen, "Raman Spectroscopy for Cancer Diagnosis. (Cover story)," Spectroscopy, vol. 21, pp. 33-41, 2006.

[43] A. Nijssen, S. Koljenović, T. C. Bakker Schut, P. J. Caspers, and G. J. Puppels, "Towards 
oncological application of Raman spectroscopy.," J. Biophotonics, vol. 2, no. 1-2, pp. 2936, Feb. 2009.

[44] J. Desroches et al., "A new method using Raman spectroscopy for in vivo targeted brain cancer tissue biopsy," Sci. Rep., 2018.

[45] M. B. Fenn, P. Xanthopoulos, G. Pyrgiotakis, S. R. Grobmyer, P. M. Pardalos, and L. L. Hench, "Raman Spectroscopy for Clinical Oncology," Adv. Opt. Technol., vol. 2011, pp. $1-20,2011$.

[46] D. Sorak, L. Herberholz, S. Iwascek, S. Altinpinar, F. Pfeifer, and H. W. Siesler, "New developments and applications of handheld raman, mid-infrared, and near-infrared spectrometers," Appl. Spectrosc. Rev., vol. 47, no. 2, pp. 83-115, 2012.

[47] N. J. Everall, “Confocal Raman microscopy: Common errors and artefacts,” Analyst. 2010.

[48] K. Klein et al., "Label-free live-cell imaging with confocal Raman microscopy," Biophys. $J ., 2012$.

[49] A. P. Ayala, "Polymorphism in drugs investigated by low wavenumber Raman scattering," Vib. Spectrosc., 2007.

[50] B. Yang, H. Owen, and M. D. Morris, "Holographic Notch Filter for Low-Wavenumber Stokes and Anti-Stokes Raman Spectroscopy," Appl. Spectrosc. Vol. 45, Issue 9, pp. 1533-1536, 1991.

[51] C. Raml, X. He, M. Han, D. R. Alexander, and Y. Lu, "Raman spectroscopy based on a single-crystal sapphire fiber," Opt. Lett., 2011.

[52] S. O. Konorov, C. J. Addison, H. G. Schulze, R. F. B. Turner, and M. W. Blades, "Hollow-core photonic crystal fiber-optic probes for Raman spectroscopy.," Opt. Lett., vol. 31, no. 12, pp. 1911-1913, 2006.

[53] I. Latka, S. Dochow, C. Krafft, B. Dietzek, and J. Popp, "Fiber optic probes for linear and 
nonlinear Raman applications - Current trends and future development," Laser Photonics Rev., vol. 7, no. 5, pp. 698-731, 2013.

[54] N. Stone, M. Kerssens, G. R. Lloyd, K. Faulds, D. Graham, and P. Matousek, "Surface enhanced spatially offset Raman spectroscopic (SESORS) imaging - the next dimension," Chem. Sci., vol. 2, no. 4, p. 776, 2011.

[55] K. Chao et al., "A spatially offset raman spectroscopy method for non-destructive detection of gelatin-encapsulated powders," Sensors (Switzerland), 2017.

[56] C. Eliasson, N. A. Macleod, and P. Matousek, "Noninvasive detection of concealed liquid explosives using Raman spectroscopy,” Anal. Chem., vol. 79, no. 21, pp. 8185-8189, 2007.

[57] B. G. Saar, C. W. Freudiger, J. Reichman, C. M. Stanley, G. R. Holtom, and X. S. Xie, "Video-rate molecular imaging in vivo with stimulated Raman scattering," Science (80-. )., 2010.

[58] K. Kong, C. Kendall, N. Stone, and I. Notingher, "Raman spectroscopy for medical diagnostics — From in-vitro bio fl uid assays to in-vivo cancer detection $\hat{\varkappa}^{2}, " A d v$. Drug Deliv. Rev., vol. 89, pp. 121-134, 2015.

[59] C. L. Evans, E. O. Potma, M. Puoris'haag, D. Cote, C. P. Lin, and X. S. Xie, "Chemical imaging of tissue in vivo with video-rate coherent anti-Stokes Raman scattering microscopy," Proc. Natl. Acad. Sci., 2005.

[60] C. Zhang, D. Zhang, and J.-X. Cheng, "Coherent Raman Scattering Microscopy in Biology and Medicine,” Annu. Rev. Biomed. Eng., 2015.

[61] D. Wei, S. Chen, and Q. Liu, "Review of fluorescence suppression techniques in Raman spectroscopy," Applied Spectroscopy Reviews, vol. 50, no. 5. pp. 387-406, 2015.

[62] E. Cordero, F. Korinth, C. Stiebing, C. Krafft, I. W. Schie, and J. Popp, "Evaluation of shifted excitation raman difference spectroscopy and comparison to computational background correction methods applied to biochemical raman spectra," Sensors 
(Switzerland), 2017.

[63] P. J. Cadusch, M. M. Hlaing, S. A. Wade, S. L. McArthur, and P. R. Stoddart, "Improved methods for fluorescence background subtraction from Raman spectra," J. Raman Spectrosc., 2013.

[64] D. Matthew and M. Elizabeth, "Raman Spectroscopy for Cancer Diagnosis," 2006.

[65] B. Schrader et al., "NIR Raman spectroscopy in medicine and biology: results and aspects," J. Mol. Struct., vol. 480-481, pp. 21-32, May 1999.

[66] S. K. Majumder, M. D. Keller, F. I. Boulos, M. C. Kelley, and A. Mahadevan-Jansen, "Comparison of autofluorescence, diffuse reflectance, and Raman spectroscopy for breast tissue discrimination.," J. Biomed. Opt., vol. 13, no. 5, p. 054009, 2008.

[67] K. E. Shafer-Peltier et al., "Raman microspectroscopic model of human breast tissue: Implications for breast cancer diagnosis in vivo," J. Raman Spectrosc., vol. 33, pp. 552$563,2002$.

[68] A. S. Haka, K. E. Shafer-Peltier, M. Fitzmaurice, J. Crowe, R. R. Dasari, and M. S. Feld, "Diagnosing breast cancer by using Raman spectroscopy.," Proc. Natl. Acad. Sci. U. S. A., vol. 102, no. 35, pp. 12371-6, Aug. 2005.

[69] K. Bräutigam, T. Bocklitz, M. Schmitt, P. Rösch, and J. Popp, "Raman spectroscopic imaging for the real-time detection of chemical changes associated with docetaxel exposure.," Chemphyschem, vol. 14, pp. 550-3, 2013.

[70] C. L. Zavaleta et al., "A Raman-based endoscopic strategy for multiplexed molecular imaging.," Proc. Natl. Acad. Sci. U. S. A., vol. 110, no. 25, pp. E2288-97, Jun. 2013.

[71] I. J. Jahn et al., "Surface-enhanced Raman spectroscopy and microfluidic platforms: Challenges, solutions and potential applications," Analyst. 2017.

[72] N. A. Abu Hatab, J. M. Oran, and M. J. Sepaniak, "Surface-enhanced Raman spectroscopy substrates created via electron beam lithography and nanotransfer printing," 
ACS Nano, 2008.

[73] M. Kahl, E. Voges, S. Kostrewa, C. Viets, and W. Hill, "Periodically structured metallic substrates for SERS," Sensors Actuators B Chem., vol. 51, no. 1-3, pp. 285-291, Aug. 1998.

[74] J. C. Hulteen and R. P. Van Duyne, "Nanosphere lithography: A materials general fabrication process for periodic particle array surfaces," J. Vac. Sci. Technol. A Vacuum, Surfaces, Film., vol. 13, no. 3, pp. 1553-1558, 1995.

[75] S. Chattopadhyay, H. C. Lo, C. H. Hsu, L. C. Chen, and K. H. Chen, "Surface-enhanced Raman spectroscopy using self-assembled silver nanoparticles on silicon nanotips," Chem. Mater., 2005.

[76] N. R. Isola, D. L. Stokes, and T. Vo-Dinh, "Surface-Enhanced Raman Gene Probe for HIV Detection," Anal. Chem., 1998.

[77] R. A. Tripp, R. A. Dluhy, and Y. Zhao, "Novel nanostructures for SERS biosensing recent progress in SERS biosensing is given in this article .," vol. 3, no. 3, pp. 31-37, 2008.

[78] T. R. Jensen, M. D. Malinsky, C. L. Haynes, and R. P. Van Duyne, "Nanosphere Lithography: Tunable Localized Surface Plasmon Resonance Spectra of Silver Nanoparticles," J. Phys. Chem. B, vol. 104, no. 45, pp. 10549-10556, Nov. 2000.

[79] J. Zhang, Y. Li, X. Zhang, and B. Yang, "Colloidal self-assembly meets nanofabrication: from two-dimensional colloidal crystals to nanostructure arrays.," Adv. Mater., vol. 22, no. 38, pp. 4249-69, Oct. 2010.

[80] A. I. Kuznetsov et al., "Laser fabrication of large-scale nanoparticle arrays for sensing applications.," ACS Nano, vol. 5, no. 6, pp. 4843-9, Jun. 2011.

[81] W. Kandulski, “Shadow Nanosphere Lithography,” University of Bonn, 2007.

[82] C. Schönenberger et al., "Template Synthesis of Nanowires in Porous Polycarbonate Membranes: Electrochemistry and Morphology," J. Phys. Chem. B, vol. 101, no. 28, pp. 
5497-5505, 1997.

[83] E. Batista, D. dos Santos, G. S. Andrade, A. Sant'Ana, A. Brolo, and M. A. Temperini, "Using Polycarbonate Membranes as Templates for the Preparation of Au Nanostructures for Surface-Enhanced Raman Scattering," J. Nanosci. Nanotechnol., vol. 9, no. 5, pp. 3233-3238, 2009.

[84] A. Kassu, C. Farley, A. Sharma, W. Kim, and J. Guo, "Effect of Pore Size and Film Thickness on Gold-Coated Nanoporous Anodic Aluminum Oxide Substrates for SurfaceEnhanced Raman Scattering Sensor,” Sensors, vol. 15, no. 12, pp. 29924-29937, 2015.

[85] H. Ko, S. Chang, and V. V. Tsukruk, "Porous substrates for label-free molecular level detection of nonresonant organic molecules," ACS Nano, vol. 3, no. 1, pp. 181-188, 2009.

[86] E. J. Bjerneld, F. Svedberg, and M. Käll, "Laser induced growth and deposition of noblemetal nanoparticles for surface-enhanced Raman scattering," Nano Lett., vol. 3, no. 5, pp. 593-596, 2003.

[87] E. D. Diebold, N. H. Mack, S. K. Doom, and E. Mazur, "Femtosecond LaserNanostructured substrates for Surface-Enhanced raman scattering," Langmuir, vol. 25, no. 3, pp. 1790-1794, 2009.

[88] L. H. Qian, X. Q. Yan, T. Fujita, A. Inoue, and M. W. Chen, "Surface enhanced Raman scattering of nanoporous gold: Smaller pore sizes stronger enhancements," Appl. Phys. Lett., vol. 90, no. 15, p. 153120, 2007.

[89] H. Liu et al., "Single molecule detection from a large-scale SERS-active Au 79 Ag 21 substrate," Sci. Rep., vol. 1, p. srep00112, 2011.

[90] D. Wang, R. Ji, A. Albrecht, and P. Schaaf, "Ordered arrays of nanoporous gold nanoparticles," Beilstein J. Nanotechnol., vol. 3, no. 1, pp. 651-657, 2012.

[91] S. Y. Lee et al., "Freestanding and arrayed nanoporous microcylinders for highly active 3D SERS substrate," Chem. Mater., vol. 25, no. 12, pp. 2421-2426, 2013. 
[92] X. Lan et al., "Surface-enhanced Raman-scattering fiber probe fabricated by femtosecond laser.," Opt. Lett., vol. 34, no. 15, pp. 2285-7, 2009.

[93] O. V Andreeva and O. V Bandyuk, "Light-Sensitive Media-Composites for Recording Volume Holograms Based on Porous Glass and Polymer,” in Holograms, I. Naydenova, Ed. Rijeka: IntechOpen, 2011.

[94] O. V Andreeva, N. V Andreeva, and T. B. Kuzmina, "Plasmonic particles of colloidal silver in high-resolution recording media," Opt. Spectrosc., vol. 122, no. 1, pp. 52-58, Jan. 2017.

[95] A. P. Litvin et al., "Strong Enhancement of PbS Quantum Dot NIR Emission Using Plasmonic Semiconductor Nanocrystals in Nanoporous Silicate Matrix," Adv. Opt. Mater., 2018.

[96] Y. Li et al., "Nanoporous glass integrated in volumetric bar-chart chip for point-of-care diagnostics of non-small cell lung cancer," ACS Nano, 2016.

[97] D. L. Stokes and T. Vo-Dinh, "Development of an integrated single-fiber SERS sensor," Sensors Actuators, B Chem., vol. 69, no. 1, pp. 28-36, 2000.

[98] C. Viets and W. Hill, "Comparison of fibre-optic SERS sensors with differently prepared tips,” Sensors Actuators B Chem., vol. 51, no. 1-3, pp. 92-99, Aug. 1998.

[99] Q. Zhou, X. Zhang, Y. Huang, Z. Li, Y. Zhao, and Z. Zhang, "Enhanced surface-enhanced Raman scattering performance by folding silver nanorods," Appl. Phys. Lett., 2012.

[100] S. Jayawardhana, G. Kostovski, A. P. Mazzolini, and P. R. Stoddart, “Optical fiber sensor based on oblique angle deposition," Appl. Opt., 2011.

[101] D. J. White and P. R. Stoddart, "Nanostructured optical fiber with surface-enhanced Raman scattering functionality.," Opt. Lett., vol. 30, no. 6, pp. 598-600, 2005.

[102] A. Micco, A. Ricciardi, M. Pisco, V. La Ferrara, and A. Cusano, "Optical fiber tip templating using direct focused ion beam milling," Sci. Rep., vol. 5, pp. 1-11, 2015. 
[103] M. Principe et al., "Optical fiber meta-tips," Light Sci. Appl., vol. 6, no. 3, pp. e16226-10, 2017.

[104] Y. Lin, Y. Zou, Y. Mo, J. Guo, and R. G. Lindquist, "E-beam patterned gold nanodot arrays on optical fiber tips for localized surface plasmon resonance biochemical sensing," Sensors (Switzerland), vol. 10, no. 10, pp. 9397-9406, 2010.

[105] Y. Liu et al., "Highly sensitive fibre surface-enhanced Raman scattering probes fabricated using laser-induced self-assembly in a meniscus," Nanoscale, vol. 8, no. 20, pp. 1060710614, 2016.

[106] I. Antohe, D. Spasic, F. Delport, J. Li, and J. Lammertyn, "Nanoscale patterning of goldcoated optical fibers for improved plasmonic sensing," Nanotechnology, vol. 28, no. 21, 2017.

[107] G. Quero et al., "Nanosphere lithography on fiber: Towards engineered lab-on-fiber SERS optrodes," Sensors (Switzerland), vol. 18, no. 3, 2018.

[108] S. Mahajan et al., "Understanding the surface-enhanced Raman spectroscopy " background,"” J. Phys. Chem. C, 2010.

[109] J. T. Hugall, J. J. Baumberg, and S. Mahajan, "Disentangling the peak and background signals in surface-enhanced Raman scattering," J. Phys. Chem. C, 2012.

[110] J. Parsons, C. P. Burrows, J. R. Sambles, and W. L. Barnes, “A comparison of techniques used to simulate the scattering of electromagnetic radiation by metallic nanostructures," $J$. Mod. Opt., vol. 57, no. 5, pp. 356-365, Mar. 2010.

[111] A. Dhawan, M. Gerhold, and T. Vo-Dinh, "Theoretical simulation and focused ion beam fabrication of gold nanostructures for Surface-Enhanced Raman Scattering (SERS)," Nanobiotechnology, vol. 3, no. 3-4, pp. 164-171, 2007.

[112] Z. Zeng, Y. Liu, and J. Wei, "Recent advances in surface-enhanced raman spectroscopy (SERS): Finite-difference time-domain (FDTD) method for SERS and sensing applications," TrAC - Trends in Analytical Chemistry, vol. 75. pp. 162-173, 2016. 
[113] I. Knorr, K. Christou, J. Meinertz, A. Selle, J. Ihlemann, and G. Marowsky, "Prediction and Optimization of Surface-Enhanced Raman Scattering Geometries using COMSOL Multiphysics," COMSOL, 2008.

[114] S. Dickreuter, J. Gleixner, A. Kolloch, J. Boneberg, E. Scheer, and P. Leiderer, "Mapping of plasmonic resonances in nanotriangles,” Beilstein J. Nanotechnol., vol. 4, no. 1, pp. 588-602, 2013.

[115] W. Ingram, Y. He, K. Stone, W. M. Dennis, D. Ye, and Y. Zhao, "Tuning the plasmonic properties of silver nanopatterns fabricated by shadow nanosphere lithography," Nanotechnology, vol. 27, no. 38, 2016.

[116] Y. Peng, C. Marcoux, P. Patoka, M. Hilgendorff, M. Giersig, and K. Kempa, "Plasmonics of thin film quasitriangular nanoparticles," Appl. Phys. Lett., vol. 96, no. 13, 2010.

[117] R. Smith, K. L. Wright, and L. Ashton, "Raman spectroscopy: An evolving technique for live cell studies," Analyst. 2016.

[118] A. Mahadevan-Jansen and R. Richards-Kortum, "Raman spectroscopy for cancer detection: a review," 2002.

[119] C. Krafft and J. Popp, "The many facets of Raman spectroscopy for biomedical analysis," Analytical and Bioanalytical Chemistry. 2015.

[120] E. Garai et al., "High-sensitivity, real-time, ratiometric imaging of surface-enhanced Raman scattering nanoparticles with a clinically translatable Raman endoscope device.," J. Biomed. Opt., vol. 18, p. 096008, 2013.

[121] A. I. Henry, B. Sharma, M. F. Cardinal, D. Kurouski, and R. P. Van Duyne, "Surfaceenhanced Raman spectroscopy biosensing: In vivo diagnostics and multimodal imaging," Analytical Chemistry. 2016.

[122] L. F. Santos, R. Wolthuis, S. Koljenović, R. M. Almeida, and G. J. Puppels, "Fiber-optic probes for in vivo Raman spectroscopy in the high-wavenumber region," Anal. Chem., vol. 77, no. 20, pp. 6747-6752, 2005. 
[123] F. Colas, D. Barchiesi, S. Kessentini, T. Toury, and M. L. D. La Chapelle, "Comparison of adhesion layers of gold on silicate glasses for SERS detection," J. Opt. (United Kingdom), 2015.

[124] W. Zhou and Z. L. Wang, Scanning microscopy for nanotechnology: Techniques and applications. 2007.

[125] N. Jalili and K. Laxminarayana, "A review of atomic force microscopy imaging systems: Application to molecular metrology and biological sciences," Mechatronics, 2004.

[126] K. Kneipp et al., "Extremely large enhancement factors in surface-enhanced Raman scattering for molecules on colloidal gold clusters," Appl. Spectrosc., vol. 52, no. 12, pp. 1493-1497, 1998.

[127] E. C. Le Ru, E. Blackie, M. Meyer, and P. G. Etchegoin, "Surface Enhanced Raman Scattering Enhancement Factors: A Comprehensive Study,” J. Phys. Chem. C, vol. 111, no. 37, pp. 13794-13803, Sep. 2007.

[128] A. Kudelski, "Raman studies of rhodamine 6G and crystal violet sub-monolayers on electrochemically roughened silver substrates: Do dye molecules adsorb preferentially on highly SERS-active sites?," Chem. Phys. Lett., 2005.

[129] COMSOL Inc., "RF module user guide v5.3a." COMSOL, Inc., Burlington, MA, USA, 2017.

[130] COMSOL Inc., "Computing the Radar Cross Section of a Perfectly Conducting Sphere," Application ID: 10332. [Online]. Available: https://www.comsol.com/model/computingthe-radar-cross-section-of-a-perfectly-conducting-sphere-10332. [Accessed: 15-Mar2019].

[131] A. D. Rakić, A. B. Djurišić, J. M. Elazar, and M. L. Majewski, “Optical properties of metallic films for vertical-cavity optoelectronic devices," Appl. Opt., 1998.

[132] A. Ishimaru, "Scattering and Absorption of a Wave by a Single Particle," in Wave Propagation and Scattering in Random Media, IEEE, 1997. 
[133] COMSOL Inc., "Scatterer on substrate." [Online]. Available:

https://www.comsol.com/model/download/563151/models.woptics.scatterer_on_substrate. pdf. [Accessed: 20-Dec-2018].

[134] L. Angeloni, G. Smulevich, and M. P. Marzocchi, "Resonance Raman spectrum of crystal violet," J. Raman Spectrosc., vol. 8, no. 6, pp. 305-310, 1979.

[135] J. F. Rusling, "Minimizing errors in numerical analysis of chemical data," J. Chem. Educ., vol. 65 , no. 10, p. 863, 1988.

[136] K. Q. Lin et al., "Size Effect on SERS of Gold Nanorods Demonstrated via Single Nanoparticle Spectroscopy," J. Phys. Chem. C, 2016.

[137] O. Ibrahim et al., "Improved protocols for pre-processing Raman spectra of formalin fixed paraffin preserved tissue sections," Anal. Methods, 2017.

[138] P. Moskovkin and S. Lucas, "Computer simulations of the early-stage growth of Ge clusters at elevated temperatures on patterned Si substrate using the kinetic Monte Carlo method," Thin Solid Films, 2013.

[139] European Bioinformatics Institute, "Chemical Entities of Biological Interest (ChEBI)," 2015. [Online]. Available: http://www.ebi.ac.uk/. 